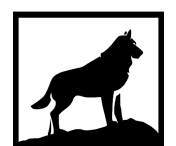

Michigan Technological

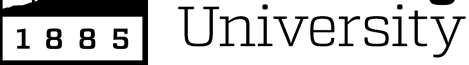

Michigan Technological University Digital Commons @ Michigan Tech

\title{
ACTIVE NOISE CONTROL USING CARBON NANOTUBE THERMOPHONES: CASE STUDY FOR AN AUTOMOTIVE HVAC APPLICATION
}

Walker H. Nelson

Michigan Technological University, wnelson@mtu.edu

Copyright 2020 Walker H. Nelson

Recommended Citation

Nelson, Walker H., "ACTIVE NOISE CONTROL USING CARBON NANOTUBE THERMOPHONES: CASE STUDY FOR AN AUTOMOTIVE HVAC APPLICATION", Open Access Master's Thesis, Michigan Technological University, 2020.

https://doi.org/10.37099/mtu.dc.etdr/1047

Follow this and additional works at: https://digitalcommons.mtu.edu/etdr

Part of the Acoustics, Dynamics, and Controls Commons 


\title{
ACTIVE NOISE CONTROL USING CARBON NANOTUBE THERMOPHONES: CASE STUDY FOR AN AUTOMOTIVE HVAC APPLICATION
}

\author{
By \\ Walker H. Nelson
}

\begin{abstract}
A THESIS
Submitted in partial fulfillment of the requirements for the degree of MASTER OF SCIENCE

In Mechanical Engineering
\end{abstract}

MICHIGAN TECHNOLOGICAL UNIVERSITY

2020

(C) 2020 Walker H. Nelson 
This thesis has been approved in partial fulfillment of the requirements for the Degree of MASTER OF SCIENCE in Mechanical Engineering.

Department of Mechanical Engineering - Engineering Mechanics

Thesis Advisor: $\quad$ Dr. Andrew Barnard

Committee Member: Dr. James DeClerck

Committee Member: $\quad$ Dr. Vijaya V. N. Sriram Malladi

Department Chair: Dr. William Predebon 


\section{Table of Contents}

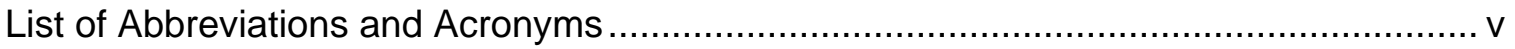

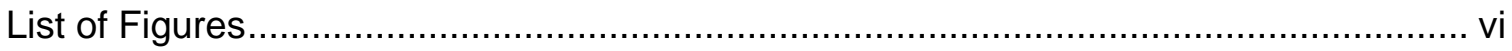

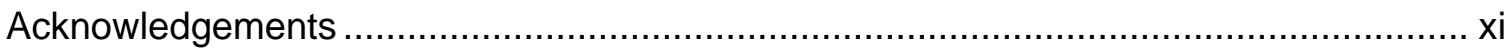

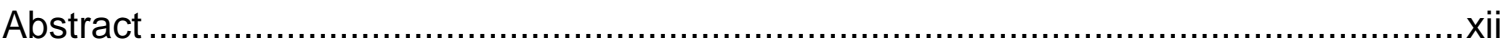

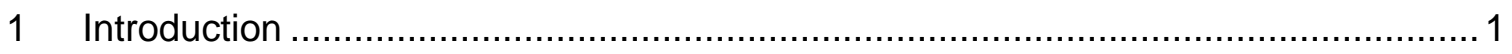

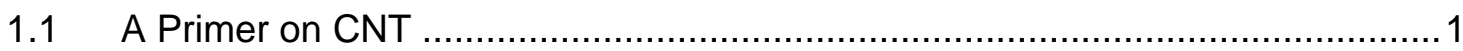

1.2 A Primer on Active Noise Control .......................................................... 2

1.3 Description of the Application Space ................................................... 6

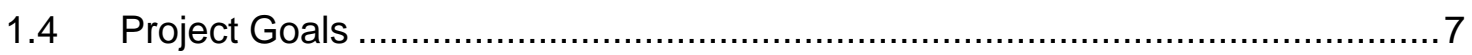

2 Materials and Methods: A Chronological Account ............................................ 8

2.1 Description of Important Test Setups....................................................... 8

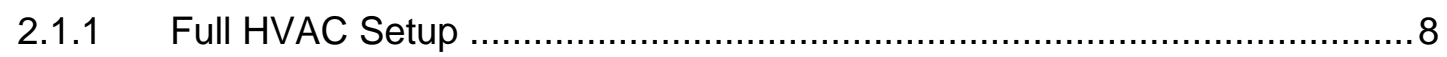

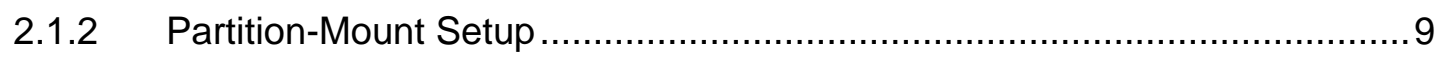

2.2 Initial Characterization of Fan Noise ............................................................ 10

2.3 Acoustic \& Temperature Performance Analysis of the RC01 Thermophone Prototype ............................................................................................... 11

2.4 Initial Acoustic \& Temperature Performance Analysis of the RC03

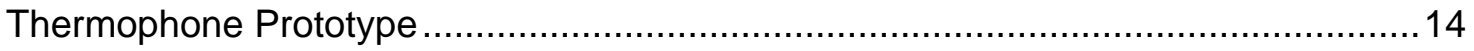

2.5 GLSV ANC Controller Performance Testing \& ANC Demo ............................18

2.6 Temperature Testing of the Kapton Encapsulated Flexible Thermophone

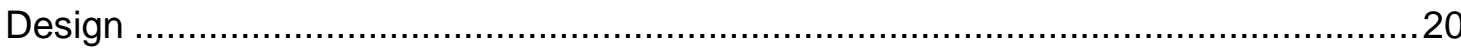

2.7 Testing to Identify the Ideal Material for Flexible Thermophone Encapsulation21

2.7.1 Acoustic and Temperature Testing for Fiberglass Encapsulated Flexible

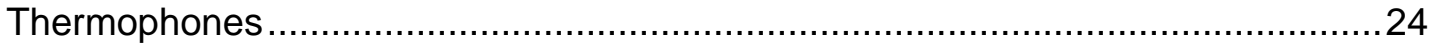

2.8 Acoustic Path Comparison Experiment ................................................28

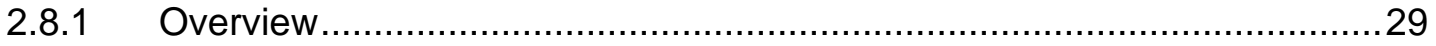

2.8.2 Test Configuration .......................................................................29

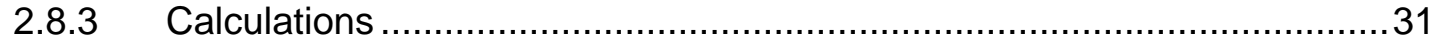

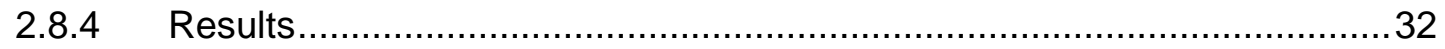

2.9 Initial Cancellation Testing with the Tiger WIFI-S Controller.........................37

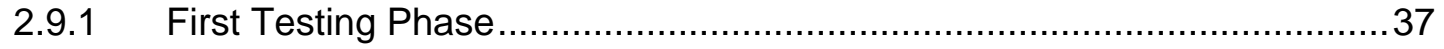

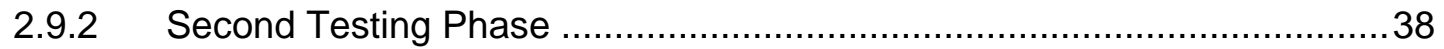




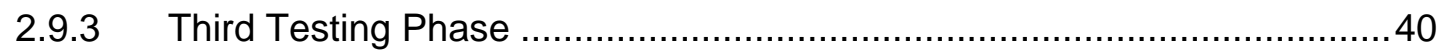

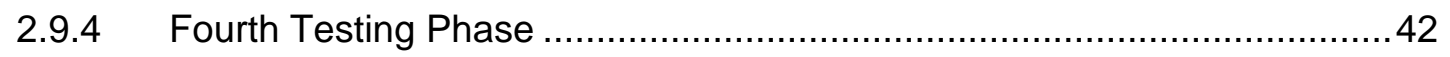

2.10 In-Duct Cancellation Testing with the Tiger WIFI-S ....................................44

2.11 Testing the Effects of Causality, Coherence, and Feedback on ANC

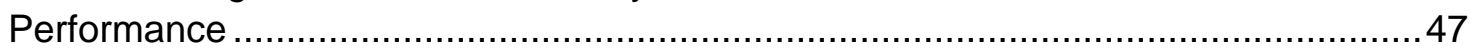

2.12 In-Duct ANC Testing with The cRIO FPGA Controller ....................................52

3 Development of an FPGA-Based ANC Controller.................................................59

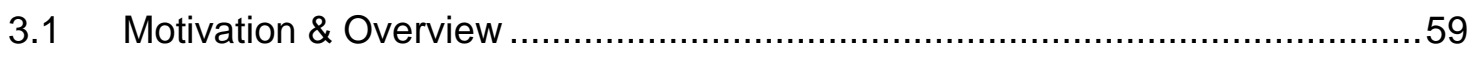

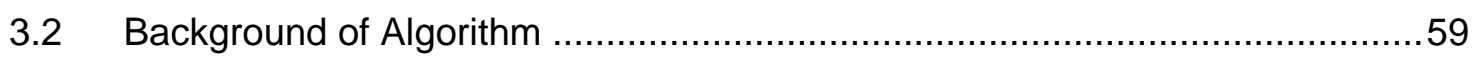

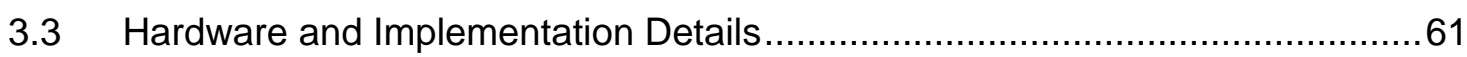

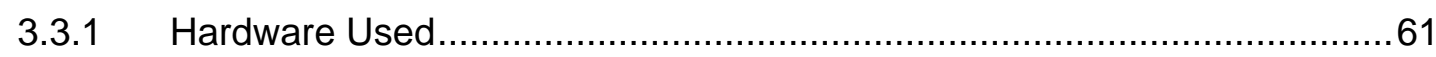

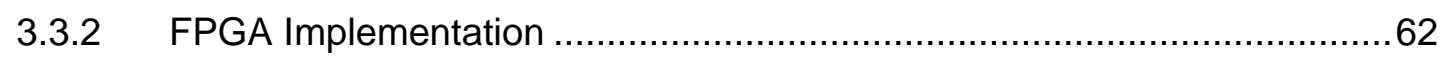

3.3.3 PC and Real-Time Components of Controller ......................................70

4 Testing Results and Interpretation: Key Findings .............................................74

4.1 A Fundamental Shift in Cancellation Strategy ...........................................74

4.2 First Analysis of Noises within the HVAC System ……................................75

4.3 Why the Tiger WIFI-S both Works and Doesn't Work...................................77

4.4 Can What Prevents the Tiger WIFI-S From Cancelling be Solved? ..................80

4.5 A Proposed Model for Use in Ducted ANC Systems Design \& What it Can Tell Us 83

4.6 Realization of The Proposed Optimal Ducted ANC System ……………......86

4.7 Comments on the Viability of the Thermophone in this Case Study .................88

4.7.1 Acoustics - Replication of the Acoustic Field From Two Sources..............88

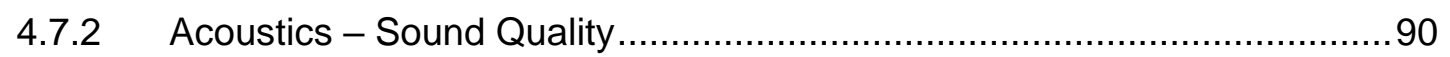

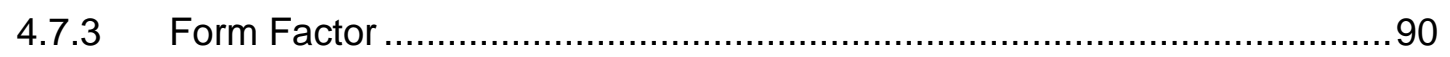

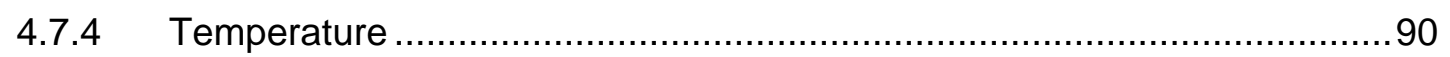

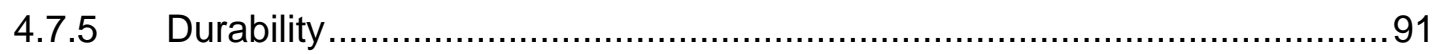

4.7.6 Cost and Manufacturability ................................................................

5 Conclusions \& Future Work ……...............................................................

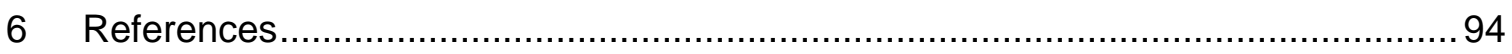

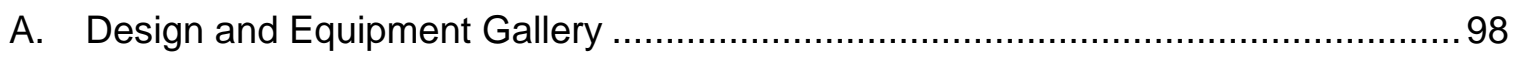




\section{List of Abbreviations and Acronyms}

\begin{tabular}{|c|c|c|}
\hline ADC & 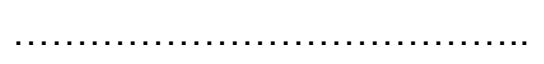 & Analog to Digital Converter \\
\hline ANC & & Active Noise Control \\
\hline CNT & & Carbon Nanotube \\
\hline FC01 & & Flexible Concept 01 \\
\hline FIFO & & First-in First-out Buffer \\
\hline FPGA & & Field Programmable Gate Array \\
\hline FRF & & Frequency Response Function \\
\hline FXLMS & & Filtered-X Least Mean Squares \\
\hline GLSV & & Great Lakes Sound and Vibration \\
\hline HCPUA & & Heat Capacity Per Unit Area \\
\hline HVAC & & Heating, Ventilation, and Air Conditioning \\
\hline IL & & Insertion Loss \\
\hline $\mathrm{RC} 01$ & & Rigid Concept 01 \\
\hline $\mathrm{RC03}$ & & Rigid Concept 03 \\
\hline SCTL & & Single-Cycle Timed Loop \\
\hline SNR & & Signal-to-Noise Ratio \\
\hline TE & & Thermoacoustic Effect \\
\hline TL & & Transmission Loss \\
\hline
\end{tabular}




\section{List of Figures}

Figure 1: CNT Thin Film Being Pulled From a Forest ................................................ 1

Figure 2: Example of Destructive Interference of Two Sound Waves in the Spatial

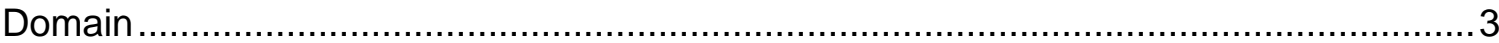

Figure 3: A Disturbance Pressure Wave Observed Over Time Will Appear to Move Across the Plot and Now Requires Tracking from a Reference Signal ...........................4

Figure 4: Interactions of 2D Sound Fields Sharing and not Sharing an Origin.................. 5

Figure 5: The HVAC System Subject to ANC System Integration in this Case Study ...... 6 Figure 6: Full HVAC Setup in MTU Anechoic Chamber With Free Field Microphone at the Standard Driver's Ear Location Used for Testing ................................................. 8

Figure 7: Installation of Blower Fan onto Partition: Fan Outlet in Anechoic Chamber ......9

Figure 8: Microphone Locations for Initial Fan Noise Benchmark Testing ......................10

Figure 9: Initial Measurements of Fan Spectra at Measurement Mic Location ...............11

Figure 10: Comparison Between the COP Spectrum of the Blower Fan to a Broadband

Output from the RC01 Thermophone....................................................................... 12

Figure 11: Thermocouple Placement For Temperature Measurements at Two Locations

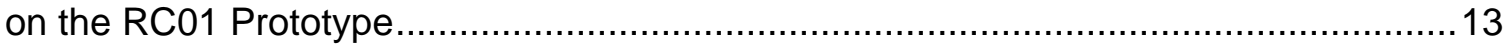

Figure 12: Steady-State Temperature of Blower Fan Plastic within CNT Chamber During

Operation of RC01 Thermophone ........................................................................13

Figure 13: Steady-State Temperature of Glass Panel Outside CNT Chamber During

Operation of RC01 Thermophone ..................................................................... 14

Figure 14: Microphone Placement for Measuring the HVAC Noise Spectra and Overall

Acoustic Output Level of the RC03 Prototype in the Full HVAC Setup..........................15

Figure 15: Acoustic Slot Required for Propagation of Sound into the HVAC from the CNT

Chamber of the RC03 Thermophone Prototype ..................................................15

Figure 16: Comparison of HVAC Noise Spectra With Two Fans Geometries Installed -

RC03 Prototype \& Nominal Fan Geometry ......................................................... 16

Figure 17: Comparison of HVAC Noise Spectra to the Broadband Output of the RC03

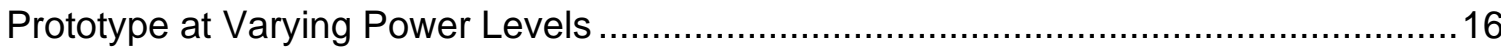

Figure 18: Highlighted Thermocouple Placements for RC03 Thermal Testing ...............17

Figure 19: Temperature at two Points on the RC03 Prototype Over Time - Varying

Power Levels and Fan Speeds .............................................................................18

Figure 20: Full HVAC Configuration with Free Field Measurement Microphone \&

Tachymeter Positioned for Tonal Cancellation Trials.

Figure 21: Comparison of Nominal HVAC Noise Spectra to Two Spectra Experiencing

Tonal Cancellation .......................................................................................... 19

Figure 22: Kapton Strip Flexible Thermophone Before and After Installation into Blower

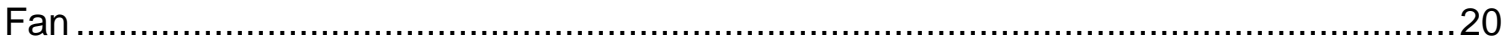

Figure 23: Temperature of Fan Surface Under Kapton Strip Flexible Thermophone

During Fan/Thermophone Operation ..............................................................21

Figure 24: Impedance Tube Used for Encapsulation Material Transmission Loss Testing

Figure 25: Setup for Measuring Output Level of Rigid Panel Thermophone for Pseudo-IL

Testing 23 
Figure 26: Rigid CNT Panel with and without Fiberglass Covering Installed 24 Figure 27: Flexible Thermophone - Rigid CNT Panel \& Flexible Thermophone Installed on ABS Panel. 25

Figure 28: Comparison of Glass Panel, Flexible on Glass, and Flexible on Plastic Thermophone Output Spectra ..........................................................................26

Figure 29: Thermocouple Location for FC01 Prototype Installation ..............................27

Figure 30: Temperature of Plastic in Blower Fan During FC01 Operation .....................28

Figure 31: Temperature of Plastic in Blower Fan During FC01 During Simulated Fan

Failure .28

Figure 32: Measurement Microphones Configured to Characterize Sources Inside the Blower Fan in the Partition-Mount Configuration 30 Figure 33: Measurement Microphones Configured to Characterize Sources Inside the Blower Fan in the Full HVAC Configuration 30

Figure 34: Detail Placement of Surface-Mount Microphone Location Used for Characterization of Sources Inside the Blower Fan in the Full HVAC Configuration ......30 Figure 35: Gain and Phase Between Responses of Mics. 2 \& 3 for Three Noise Sources in the Partition-Mount Setup...

Figure 36: Gain and Phase Between Responses of Mics. 2 \& 3 for Two Noise Sources in the Partition-Mount Setup 34

Figure 37: Comparison of FRF Calculation Methods for Different Noise Sources in the HVAC Setup. 35

Figure 38: Comparison of FRF Calculation Methods for Blower Fan Noise in the

Partition-Mount Setup 36

Figure 39: Comparison of Deterministic Magnitude Spectra to Autopower Spectra for Two Noise Sources in the Partition-Mount Setup 36

Figure 40: Comparison of Deterministic Magnitude Spectra to Autopower Spectra for Two Noise Sources in the Full HVAC Setup.... 37

Figure 41: Microphone, Disturbance, and Actuator Placement for the First Free-Field ANC Tests Performed with the Tiger WIFI-S Controller.

Figure 42: Microphone, Disturbance, and Actuator Placement for the First Free-Field ANC Tests Performed On Blower Fan Noise with the Tiger WIFI-S Controller. 39

Figure 43: Cancellation of Blower Fan Noise with Loudspeaker Actuator - Tiger WIFI-S

Cancellation Testing Phase 02

Figure 44: Microphone, Disturbance, and Loudspeaker Actuator Placement for Free-

Field ANC Tests Performed On Blower Fan Noise with the Tiger WIFI-S Controller......40 Figure 45: Microphone, Disturbance, and Thermophone Actuator Placement for FreeField ANC Tests Performed On Blower Fan Noise with the Tiger WIFI-S Controller......40 Figure 46: Pre and Post ANC Spectra Showing Cancellation of Fan Noise in the Free Field using a Loudspeaker Actuator and the Tiger WIFI-S Controller.

Figure 47: Sample Spectrum from Phase 03 ANC Testing - CNT Actuator

Figure 48: Tachymeter, Microphone, Disturbance, \& Actuator Configuration for Cancellation of HVAC Noise at a Free-Field Location

Figure 49: Detail of Loudspeaker Disturbance Placement on Blower Fan.

Figure 50: Comparison of Microphone Coherence Before and After Tuning Microphone

Spacing 
Figure 51: Configuration of Demo Ducted Setup for Cancellation Testing with a Loudspeaker Disturbance \& CNT Actuator ....

Figure 52: Configuration of Ducting Setup for Cancellation Testing with a Blower Fan Disturbance.

Figure 53: Best-Case Scenario for Broadband Cancellation in a Ducted Environment Reference Signal Acquired from a Direct Electrical Line to the Disturbance Loudspeaker

Figure 54: In-Duct Broadband Cancellation with Marginal Effectiveness -Reference Signal Acquired from a Flush-Mounted 1/4" Microphone Within the Ducting.... 46 Figure 55: Comparison of Microphone Coherence with Differing Mylar Film Applications and Blower Fan Speeds

Figure 56: Microphone, Disturbance, and Actuator Configuration for Cancellation of Loudspeaker-Generated Noise in the Free Field

Figure 57: Example of Free-Field Cancellation Stand with Two Reference/Error Microphone Spacings (20 \& $12 \mathrm{~cm})$ 48

Figure 58: Example of Cancellation Levels at the Error Microphone for the Free-Field Cancellation Stand with For $20 \mathrm{~cm}$ (right) and 12cm (left) Microphone Spacings.

Figure 59: Cancellation Levels Achieved using the Free Field Cancellation Stand at Various Microphone Spacings - Loudspeaker Disturbance

Figure 60: Free Field Cancellation Stand Placed to Cancel Noise from the Blower Fan in the Partition-Mount Configuration . 50

Figure 61: Noise Reduction Achieved with the Free Field Cancellation Stand for Two Trials with Different Levels of Microphone Coherence - Blower Fan Disturbance .........50 Figure 62: Microphone, Disturbance, and Actuator Configuration in a Ducted Setup for Coherence vs. Cancellation Experimental Trials

Figure 63: Best and Worst Case Results for Cancellation of White Noise in a Duct With

Differing Levels of Airflow.....

Figure 64: Plastic Film Covering the Microphone Flush Mount Hole to Improve Coherence.... .52

Figure 65: Microphone, Disturbance, and Actuator Configuration in the Ducted PartitionMount Test Setup for Cancelling Noise with the Annular Loudspeaker Array.....

Figure 66: Cancelling a Tonal Disturbance with the cRIO FPGA Controller and the Annular Aluminum Thermophone Actuator ....

Figure 67: Cancelling a Tritone Disturbance with the cRIO FPGA Controller and the Annular Aluminum Thermophone Actuator .

Figure 68: Cancellation of Broadband Noise with the cRIO FPGA Controller and the Annular Aluminum Thermophone Actuator....

Figure 69: Cancellation of an $800 \mathrm{~Hz}$ Tone Using the Dynamic Step Size Feature of the cRIO FPGA Controller and the Annular Aluminum Thermophone Actuator...

Figure 70: Cancellation of a Broadband Disturbance Using the cRIO FPGA Controller with the Annular Loudspeaker Array Actuator and a Microphone Providing the Reference

Figure 71: Cancellation of a Broadband Disturbance Using the cRIO FPGA Controller with the Annular Loudspeaker Array Actuator and a Reference Signal Acquired Directly from the Loudspeaker Disturbance 
Figure 72: Spectra at the Error Microphone Location Before and After the Application of ANC using the cRIO FPGA Controller and the Annular Loudspeaker Array Actuator -Coherence Between Error and Reference Microphones Before Application of ANC.....57 Figure 73: Cancellation of a Tonal Disturbance Played Over Fan Noise with the cRIO FPGA Controller \& the Annular Loudspeaker Array Actuator .... .58

Figure 74: Time-Domain FXLMS Algorithm with Feedback Neutralization .....................60 Figure 75: Sequential Filtering and Filter Update Operations of the Time-Domain FXLMS

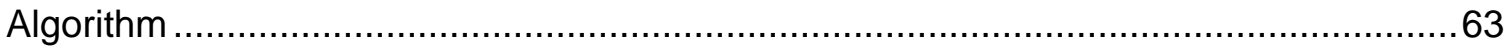

Figure 76: 16 Elements Sharing Block-Scoped Addresses are Read from a Vector ......65 Figure 77: Writing A Single Element to a Circular Buffer and Incrementing the Write Address.....

Figure 78: Converting the Block-Scoped Temporal Address into the Block-Scoped

Spatial Address 66

Figure 79: Rearranging the 16 Elements Retrieved from a Circular Buffer for GroupScoped Temporal Alignment.... 67

Figure 80: Overview of Mathematical Engine .... 68

Figure 81: Data Path for Dot Product Operation .....................................................68

Figure 82: Vector Element Retrieval and Mathematical Engine in a Pipelined Single-

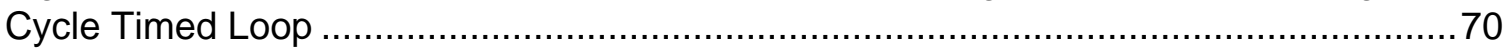

Figure 83: Controller Characterization Page ................................................................ 71

Figure 84: Controller Cancellation Page ................................................................... 72

Figure 85: Expected Results from a Causality-Centered Cancellation Experiment ........78

Figure 86: Example of The Relationship Between Coherence and Free-Field

Cancellation Levels for an Arbitrary Band ............................................................ 79

Figure 87: Example of The Relationship Between Coherence and in-Ducting Cancellation Levels for an Arbitrary Band....

Figure 88: Selection of Bands to be Targeted by Cancellation for Maximum Impact on the Overall SPL

Figure 89: Relationship between Coherence and Free-Field Cancellation Levels for two

Frequency Bands

Figure 90: Best-Case Coherence of Blower Fan Noise across Two Bands in a Ducted High-Airflow Setup

Figure 91: Direct and Reflected Waves Propagating Away from a Point Source in a Duct

Figure 92: Separation of Ducting Containing a Point Source into Two Zones Containing Different Wave Types.

Figure 93: Definition of Zones Relative to the Duct Exit Which Contain Either Cancellable or Non-Cancellable Point Sources 85 Figure 94: Annular Loudspeaker Array and Annular Aluminum Thermophone Installed in 4" PVC Ducting for Noise Cancellation 86 Figure 95: Cancellation Levels Achieved within a Ducted Environment for Disturbances which Varied in their pre-ANC SPL at $1 \mathrm{~m}$ from the duct outlet

Figure 96: Rigid CNT Panel Renderings - Callout and Exploded Views .98

Figure 97: A Four-Layer Composite CNT Thermophone Before Application of the Final Encapsulation Layer. 
Figure 98: Completed Four-Layer Composite CNT Thermophone using Kapton Film for Encapsulation

Figure 99: Four Different Representations of the RC01 Prototype Thermophone ........100 Figure 100: Renderings of the Initial RC03 Thermophone Prototype Geometry Integrated into the Blower Fan 101

Figure 101: Glass Rigid CNT Panel Installed onto RC03 Prototype Thermophone Using a 3D-Printed Adapter 102

Figure 102: FC01 Thermophone Prototype Before and After Installation into Blower Fan 103

Figure 103: Annular Teflon Speaker in Two Test Configurations.................................. 104

Figure 104: Close up of Annular Teflon Speaker Construction .................................... 105

Figure 105: Construction of the Annular Aluminum Thermophone ..............................106

Figure 106: Design of Annular Loudspeaker Array ................................................... 107

Figure 107: First Edition of the GLSV Order Based ANC Controller ............................109

Figure 108: cRIO 9031 Configured to Serve as an ANC Controller ..............................110 


\section{Acknowledgements}

Throughout this project, there were many people who helped me manage my stress, resources, time, and expectations. Without the differing support offered by all of them, this project certainly would not have been possible.

Of course, thank you to Marelli USA Inc. for sponsoring this project. Specifically, thanks to David, Tomasz, Ryan, and Aaron for being excellent partners on the case study, and for sitting through all of my confusing and long-winded tangents about math which sometimes I didn't even understand.

To my friends and colleagues Steff Vaglica, and Steven Senczyszyn; you two are the reason I got into this fun mess called grad school in the first place. Not sure if I should be thanking you for this now that I think about it again...

Dr. Barnard, thank you for never hesitating to tell me I did / said something stupid, but for always doing so in the nicest way possible and with a solution quick to follow.

Finally, thanks to my parents, who have been unwavering in their support of me pursuing my own mixed bag of interests in life, regardless of how random they might seem or where they have taken me. Thank you for encouraging my curiosity and providing me with the absolute freedom to determine my own purpose. 


\section{Abstract}

The goal of this project was to reduce the overall noise levels emitted by the HVAC components in a vehicle's cabin. More specifically, the feasibility of achieving this goal using two key technologies was investigated.

The first of these technologies, Active Noise Control (ANC), is a noise attenuation technique that relies on destructive interference that "cancels" unwanted noise. Typically used in situations where physical constraints prevent passive attenuation techniques from being used, ANC is known for its high size-to-effectiveness ratio. This benefit cannot be gained without a cost however; the complexity of ANC systems is significantly higher than their passive counterparts. This is due to the signal processing and actuator designs required. These actuators often take the form of moving-coil loudspeakers which, while effective, are often bulky. Because of this they are difficult to "drop in" to an existing system.

This is where the second technology comes in. Carbon Nanotube (CNT) Thermophones are solid-state speakers that operate by using rapid heat fluctuations to create sound. Called the "thermoacoustic effect," (TE) the theory of this operating principle dates to the turn of the $20^{\text {th }}$ century. Useful demonstration of TE did not occur until 2008, however, when researchers first developed the first CNT thermophones. The hallmark characteristics of these transducers are their small size and flexible nature. Compared to traditional loudspeakers they have a much smaller form factor and are more versatile in terms of where they can be placed in a cramped system.

The marriage of CNT transducers to ANC technology shows promise in improving the application space and ease of installation of ANC systems. Getting these two to cooperate, however, is not without challenges.

A case study for this union is presented here; the application space being the ducted environment of vehicle HVAC systems. 


\section{Introduction}

\subsection{A Primer on CNT}

Carbon Nanotubes (CNT) themselves are an amazing material with a myriad of incredible uses [1, 2]. To us as ANC system designers, and for the purposes of this report, however, they are considered in only one of their many forms. The thin-film variety of CNT appears as an incredibly delicate fabric. Imagine the sheerest, thinnest, black silk you can, one that is almost transparent, and that would float on air like a wisp of smoke. This is what CNT thin film is like (Figure 1).

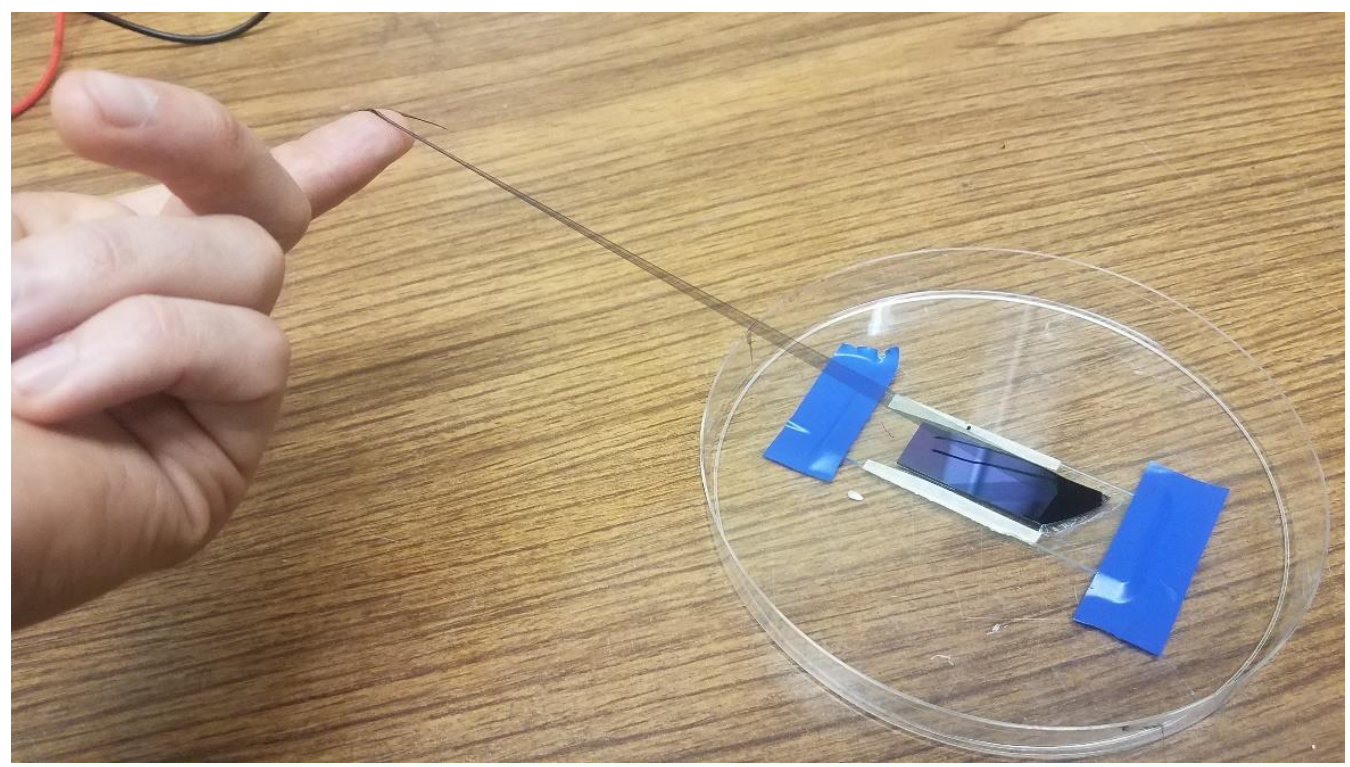

Figure 1: CNT Thin Film Being Pulled From a Forest

To produce these thin films, they are pulled from what is called a "forest." A forest is just a thicker version of the CNT film that is grown onto some substrate, typically a glass slide. Inside the forest, long chains of CNT stand on end and are interwoven. When the end of this forest is pulled, the chains of CNT get ripped away from the substrate in a way that aligns them end-to-end The result is a one nanotube thick mesh of carbon that we see as a thin film [3-5]. What makes this thin film so special though, and how can it be used to create sound?

Because of its physical makeup, the thin film is nearly massless when considered on a per unit area basis. Along with this incredibly low mass comes an incredibly low heat capacity per unit area (HCPUA) [6, 7]. What this means is that very little energy is required to change the temperature of the nanotube film. The energy requirements for temperature changes are so low in fact, that energy delivered via an alternating electrical current can fluctuate the film's temperature at frequencies in the range of hundreds of thousands of times per second. It is through these rapid fluctuations of surface temperature that sound is produced [8]. This phenomenon was first explored at the turn of the $20^{\text {th }}$ century, when 
it was discovered, and then used to create the first thermophone specifically meant to be an acoustic source $[9,10]$.

Consider a volume of air surrounding the CNT film while the film's temperature changes. Through conduction, the air particles in contact with the film also experience temperature fluctuations. According to the ideal gas law, changing the temperature of a gas will also yield a change in its density, and more importantly, in its pressure. These pressure fluctuations will then propagate through the air as sound [11].

It can be seen then, that electrical energy used to fluctuate the temperature of the CNT film is transferred into the air, converted into pressure, and can then be experienced as audible noise. Applying this electrical energy to the CNT film is simple. Since the film is pure carbon, electrically, it is essentially a pure resistor. Stretching the film physically between two electrodes is enough to make a speaker. When an alternating current is applied to the electrodes, the electrical energy is then delivered to the CNT film. Because the bare-minimum configuration for creating a speaker in this way is "a film touching two electrodes," very creative and irregular form factors can be realized.

The small and flexible transducer form factors that are possible when designing CNT are where the materials true advantages lie. This does not come for free, however. CNT has three disadvantages which must also be considered for design. The first is that thermophones, operating directly through heat, tend to generate extremely high temperatures at their surface [12]. The only saving grace in this respect is that thanks to their low HCPUA, their high temperatures do not translate into the storage of much thermal energy. Also owing to their production of much waste heat, thermophones are very inefficient at producing sound [13]. A thermophone will require much more electrical power than a traditional loudspeaker in order to produce the same sound.

Finally, thermophones also require special signal processing in order to produce intelligible sound [14]. The sound pressure produced by a loudspeaker is proportional to the voltage delivered across the speaker's armature. For CNT, the sound pressure produced is proportional to the power delivered to the film. For an AC signal across a pure resistor, power is delivered at twice the frequency of voltage or current. This can be seen easily by representing power as proportional to the square of an alternating current and then applying a trigonometric identity.

$$
P \propto I *(\sin (\omega t))^{2}=I * \frac{1}{2}(1-\cos (2 \omega t))
$$

So, CNT will double the frequency content of any signal sent to it.

\subsection{A Primer on Active Noise Control}

Active noise control, as its name suggests, is a technique used for reducing the overall levels of unwanted noise. Unlike passive noise control, which often relies on absorption or reflection of sound using acoustic materials, ANC relies on the destructive interference of pressure waves [15]. Because this technology circumvents the use of passive acoustic 
materials, it can be a very space-efficient noise control solution, especially at low frequencies. This advantage has made it attractive for a variety of applications where space is limited. A first obvious example of this are noise-cancelling headsets, which typically employ the technology using the existing loudspeakers in over-ear headphones [16]. Significant attention has also been given to ANC as a solution for noise-control problems in the passenger cabins of different types vehicles. Implementations have been investigated for yachts, turboprop airplanes, passenger cars, and helicopters [17-20]. Another application space which is highly relevant to this thesis and has been the subject of a lot of ANC research is in HVAC [21-23]. Under the right conditions, HVAC ducting can become a one-dimensional acoustics problem, making the application of ANC simple. Additionally, in many cases unwanted HVAC noise is also dominated by low frequencies or periodic noise, which are easily dealt with via ANC.

To demonstrate how ANC works, we'll walk through the simplest case possible. Imagine a single sine wave which represents acoustic pressure vs. distance. We'll call this sine "the disturbance." Picture it on a plot all by its lonesome, starting at the origin and extending some distance in time to the right or left. Now add a second sine wave, but for this one, make it a reflection of the first about the horizontal axis. This sine will be called "the control." If we were to add these two sine waves up, we would find that the result is zero at all points along the graph. In the context of the sines representing acoustic pressure, this demonstrates how ANC reduces noise levels (Figure 2).

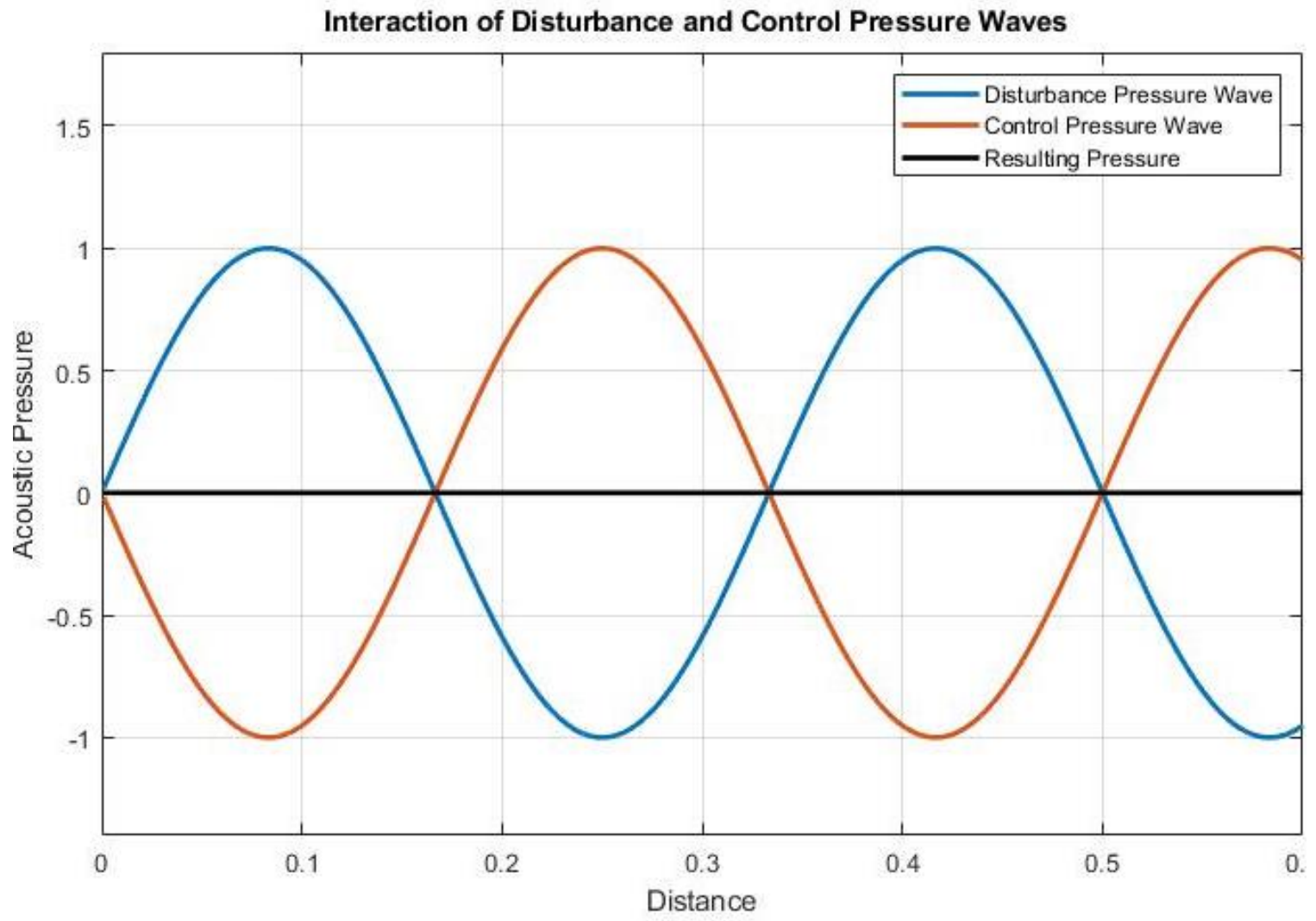

Figure 2: Example of Destructive Interference of Two Sound Waves in the Spatial Domain 
This exercise represents an extremely simplified case of any real acoustic environment, but it gets the ball rolling on understanding how ANC works, so let's stick with it for now barring a few changes. Go back to the plot with an isolated disturbance wave, but this time imagine what would happen if you observed the plot over some arbitrary time period. Pressure at a fixed location in a sound field changes with respect to time, so the disturbance would "scroll" across the plot or appear to fluctuate like a vibrating string (Figure 3).

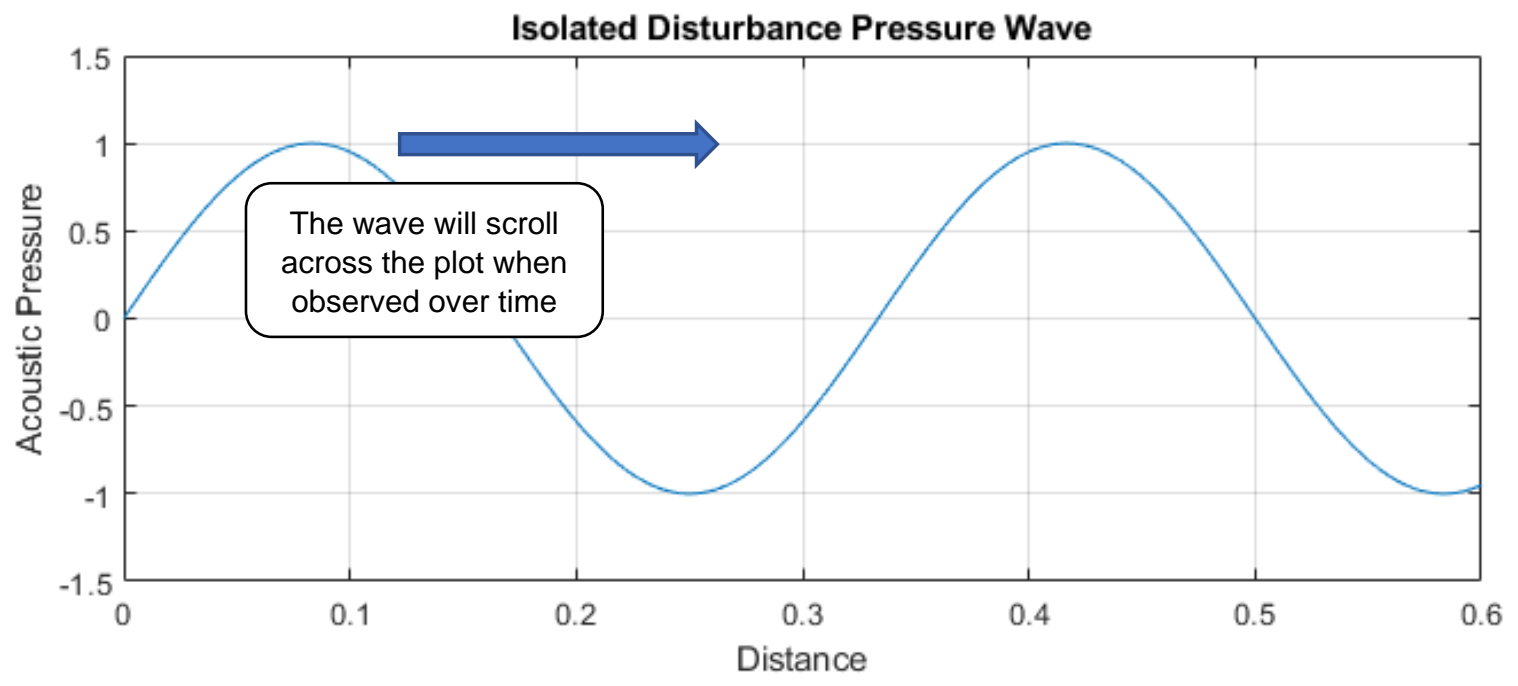

Figure 3: A Disturbance Pressure Wave Observed Over Time Will Appear to Move Across the Plot and Now Requires Tracking from a Reference Signal

Now that our disturbance is moving it is not so simple to produce a control that will destructively interfere with it; the control will need to move as well. In order to guide the movement of the control, you need to measure the movement of the disturbance. This is the job of the ANC controller.

An ANC algorithm constantly measures some portion of the disturbance and then outputs its own signal, the control. The control signal is synthesized by performing mathematical operations on the disturbance and is ultimately just a modified version of this disturbance. Algorithms start by making an initial guess as to how to best modify the disturbance in order to output a control. Based on the summation of these two signals, which may not destructively interfere perfectly at first, the controller then changes its guess as to how it should modify the disturbance. Eventually, the controller will converge to the correct modification and will lock on to producing a control that is the opposite of the disturbance.

Extend the concepts from the three prior paragraphs to a three-dimensional sound field and you have a very simple model of how an ANC system functions in a real application. The most common example of this by far are noise-cancelling headphones. In these headphones a disturbance sound wave travels from its source, through the headphones, and to your ear. As this noise travels, it is measured by a microphone, run through an ANC algorithm, and a control noise is played through the headphones to cancel it. Physically, you hear this as a reduction in unwanted noise when you turn on the ANC feature of your headphones [16]. 
Since the physical mechanisms behind ANC have been well understood for some time, most modern research has been devoted to improving the algorithms available to ANC system designers [24-27]. Of fundamental importance among these algorithms is the Filtered-X Least Mean Squares (FXLMS) algorithm [28].

With the exception of the FXLMS algorithm, minute details of these different algorithms will not be discussed in this report but it should be noted by the reader that many such algorithms exist, each with different benefits and drawbacks [29]. To learn more about the signal processing and math used in these different algorithms, consider reading Active Noise Control [30]. This textbook is extremely thorough in its derivations and explanations.

Hopefully this section has provided a good first look at how ANC works on paper. Before continuing, however, it seems pertinent to quickly consider how the addition of a third dimension affects ANC. The two-dimensional case is very simple, as it is essentially always possible to modify a signal to get it's opposite. The origins of the disturbance and control are not important if two dimensions are considered (assuming the sine waves are centered about the horizontal axis), and in fact it is strange to think of periodic signals as having an origin at all.

This changes drastically when a third dimension is added. To see why, think of a still body of water. Disturbing the surface with a falling water droplet will result in a circular wave that travels outward from a single point. If we wanted to cancel this completely how could it be done? A second circular wave must be generated obviously, but the location of the origin for this second wave is now critically important. In order to completely cancel the whole disturbance in this case, the control must share its origin point with the disturbance $[31,32]$. If this is not the case, only local cancellation at locations where the two wave fronts cross is attainable (Figure 4).
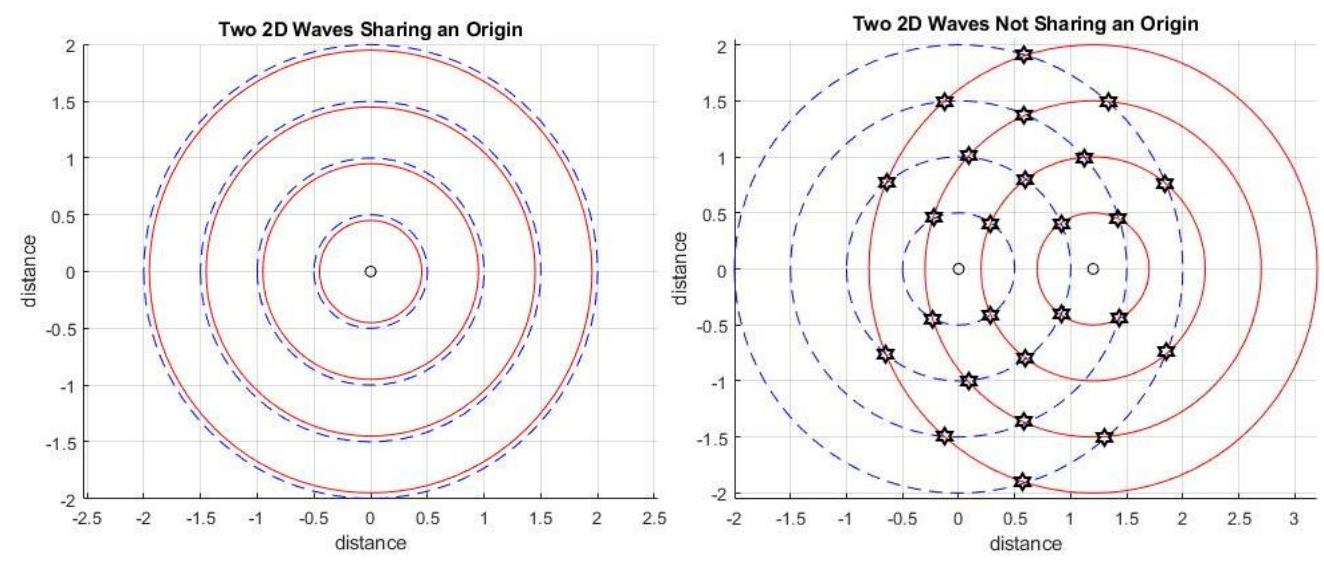

Figure 4: Interactions of 2D Sound Fields Sharing and not Sharing an Origin

This phenomenon carries serious ramifications for the design of ANC systems in three dimensions and is explored indirectly later. 
There are other specifics of ANC control and theory which would provide extra context to the reader, but whose explanations are beyond the scope of this report. I would again point the interested reader to "Active Noise Control" [30] as a starting point for deeper research into this topic.

\subsection{Description of the Application Space}

Now that a background understanding of the technology behind this project has been established, the application space can be defined. The environment set to have ANC technology integrated within it is the frontal HVAC system of a passenger vehicle. Provided by the project sponsor, the HVAC consists of many components but can be boiled down to three major groups (Figure 5). These component groups are: the Blower Fan, the evaporator / mixing chamber, and the ducting pieces.

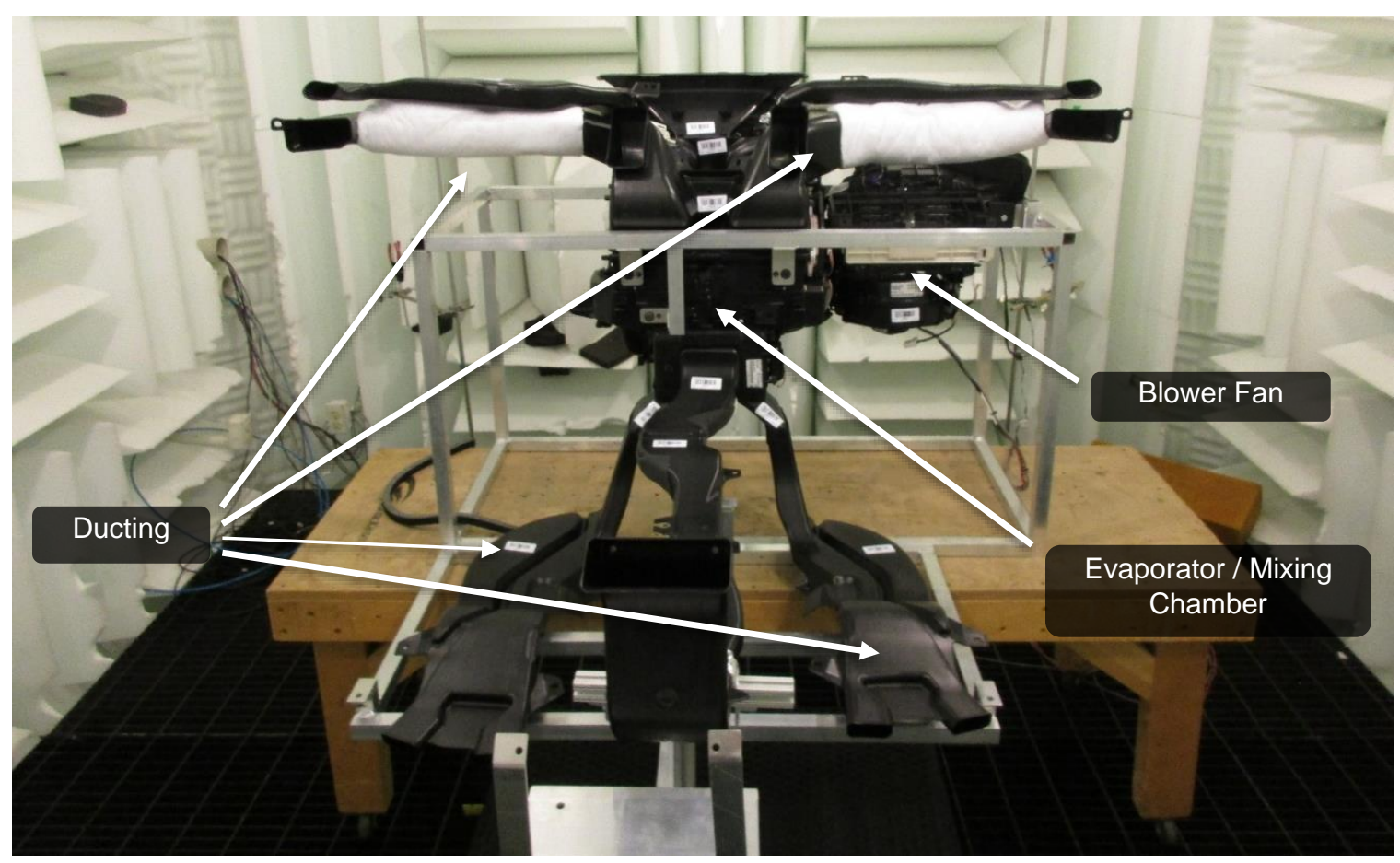

Figure 5: The HVAC System Subject to ANC System Integration in this Case Study (Photo Provided by Project Sponsor)

These pieces all fit under the dashboard of a passenger sedan and are responsible for the heating and cooling of the vehicle's cabin. The blower fan pushes air through the system, the evaporator / mixing chamber changes the temperature of the air and directs it, and the ducting delivers the air to the cabin.

Because it must meet interior comfort requirements of vehicle passengers regardless of wide-ranging exterior conditions, the system can produce very high airflow levels. In certain HVAC modes, the noise levels resulting from this extreme airflow are high when 
compared to other noises within the vehicle $[33,34]$. It is this noise, which is emitted from the HVAC system, that is the ultimate target for cancellation by the ANC system designed in this project.

\subsection{Project Goals}

After making it through the CNT, ANC, and application space the formal project statement can be stated explicitly:

The goal of this project is to investigate the feasibility of using carbon nanotube thermophones as the actuator for an active noise control system designed to reduce overall noise levels emitted from the front HVAC system of a passenger vehicle.

This motivation statement can be broken down further into two sub-goals. The first of these is to build an ANC system prototype for the project's sponsor. Secondly, the project also seeks to evaluate the performance of CNT thermophones as actuators for ANC.

To elaborate on the first sub-goal, the original design target for the ANC system was to reduce the overall SPL at the driver's ear location by $10 \mathrm{dBA}$. Additional design criteria were to: reduce the cost/size of the thermophones, characterize the thermal impacts thermophones might make on the HVAC, and optimize location for the thermophones to minimize the chances of CNT damage. As the case study progressed, the very broad initial design scope became more focused. Due to the complicated nature of the HVAC system, it was realized that the timeframe allotted could not yield an ANC system which meets all the above criteria. Instead, after some discussion, the design of an ANC demo in a limited test bench became the final project target. The test bench provides an analog to one of the subsystems the overall HVAC was split into later in the project, namely a high-airflow ducted environment.

Throughout the design of the HVAC system in this case study the effectiveness of CNT thermophones for use in ANC systems was judged. This judgement was broken down into several categories which are commented on in this report: acoustics, form factor, temperature, durability, \& cost/manufacturing.

In the acoustic evaluation, the harmonic distortion of CNT was considered from a sound quality perspective. The sound pressure levels attainable from various thermophone prototypes were also compared to the levels required of the actuator for HVAC ANC. Physical durability of different thermophone designs were evaluated from both an enclosure geometry and substrate material perspective. Temperature testing was performed to ensure that CNT actuators would be safe to use in a plastic HVAC environment. The advantages and disadvantages of the flexible CNT form factor were considered, specifically for their use in HVAC systems. And, finally, a brief explanation of the current status of CNT in terms of price and mass-manufacturing potential was covered. 


\section{Materials and Methods: A Chronological Account}

\subsection{Description of Important Test Setups}

Many test setups were used throughout the testing performed for this case study, but most of them can be described as variants of two essential setups. These are explained here; variations will be noted when necessary to describe setups for individual tests later.

\subsubsection{Full HVAC Setup}

This setup is essentially the entire HVAC system isolated within an anechoic environment. Its purpose is to be a close analog to the experience a passenger has when hearing HVAC in a vehicle cabin. Design for the setup is a replication of one of the test fixtures used at the sponsor facility (Figure 5). Components that make up the HVAC are suspended within an aluminum 8020 box in the same configuration as when installed in a vehicle (Figure 6).

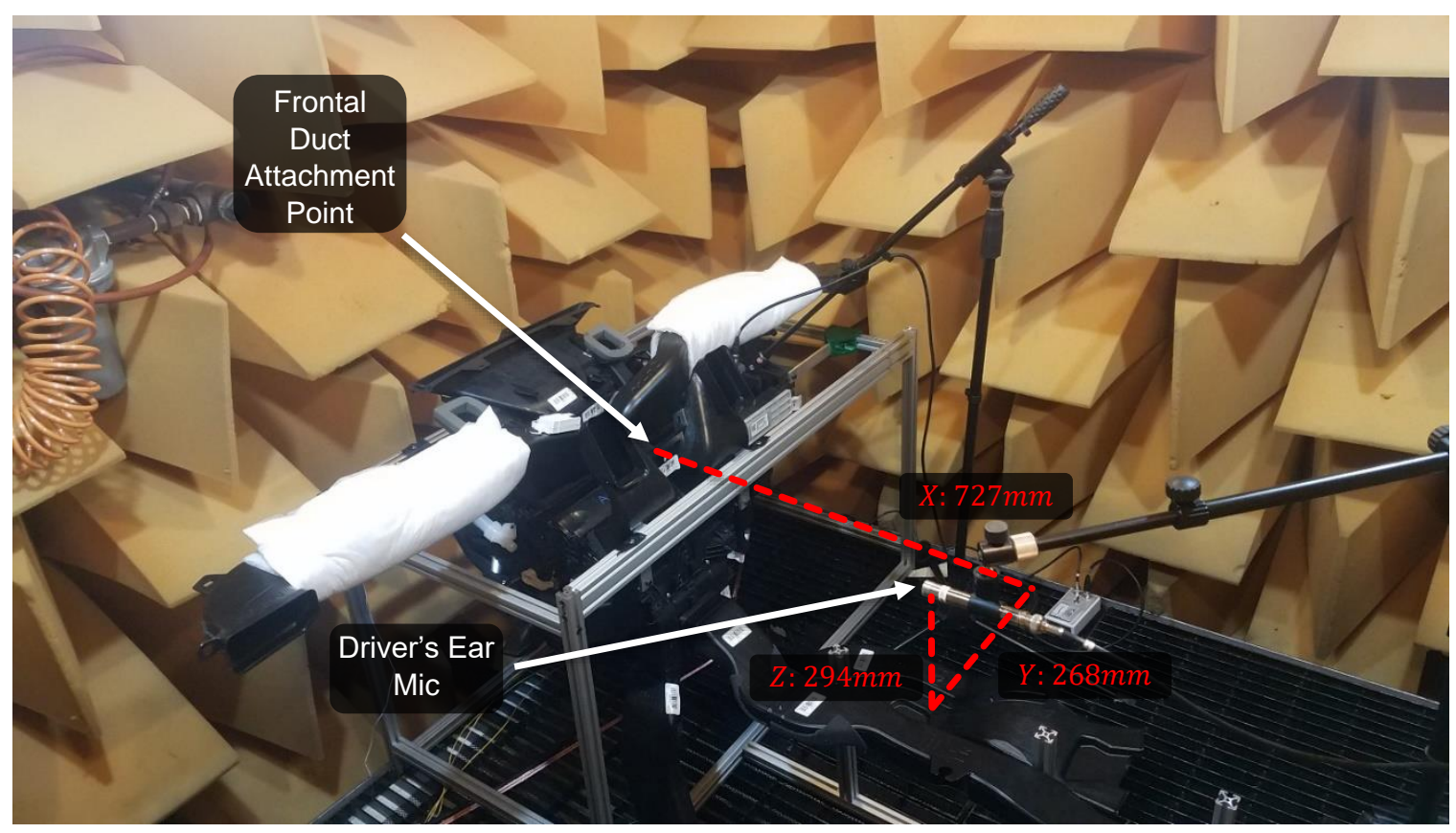

Figure 6: Full HVAC Setup in MTU Anechoic Chamber With Free Field Microphone at the Standard Driver's Ear Location Used for Testing

The evaporator / mixing chamber settings can be adjusted, but unless otherwise noted, recirculation mode was used for testing. Microphone placement varied across experiments as well, but consistently one microphone was placed $1 \mathrm{~m}$ from the HVAC center at the standard driver's ear location provided by the project sponsor (Figure 6). 


\subsubsection{Partition-Mount Setup}

The purposes for using the partition-mount setup for testing are twofold. First, this setup isolates the blower fan from the remainder of the HVAC system. Second, the partition isolates the fan's intake noise from its exhaust noise. To do this, the fan is mounted on a partition so that it blows air through a window meant to couple two isolated acoustic chambers. This isolation is important because for early designs of the ANC system it was required that the actuator be installed in the fan; in this configuration only noise generated in the fan can be cancelled. Because of this, the fan blows air into an anechoic chamber where free-field measurements of only this cancellable noise can be taken (Figure 7).
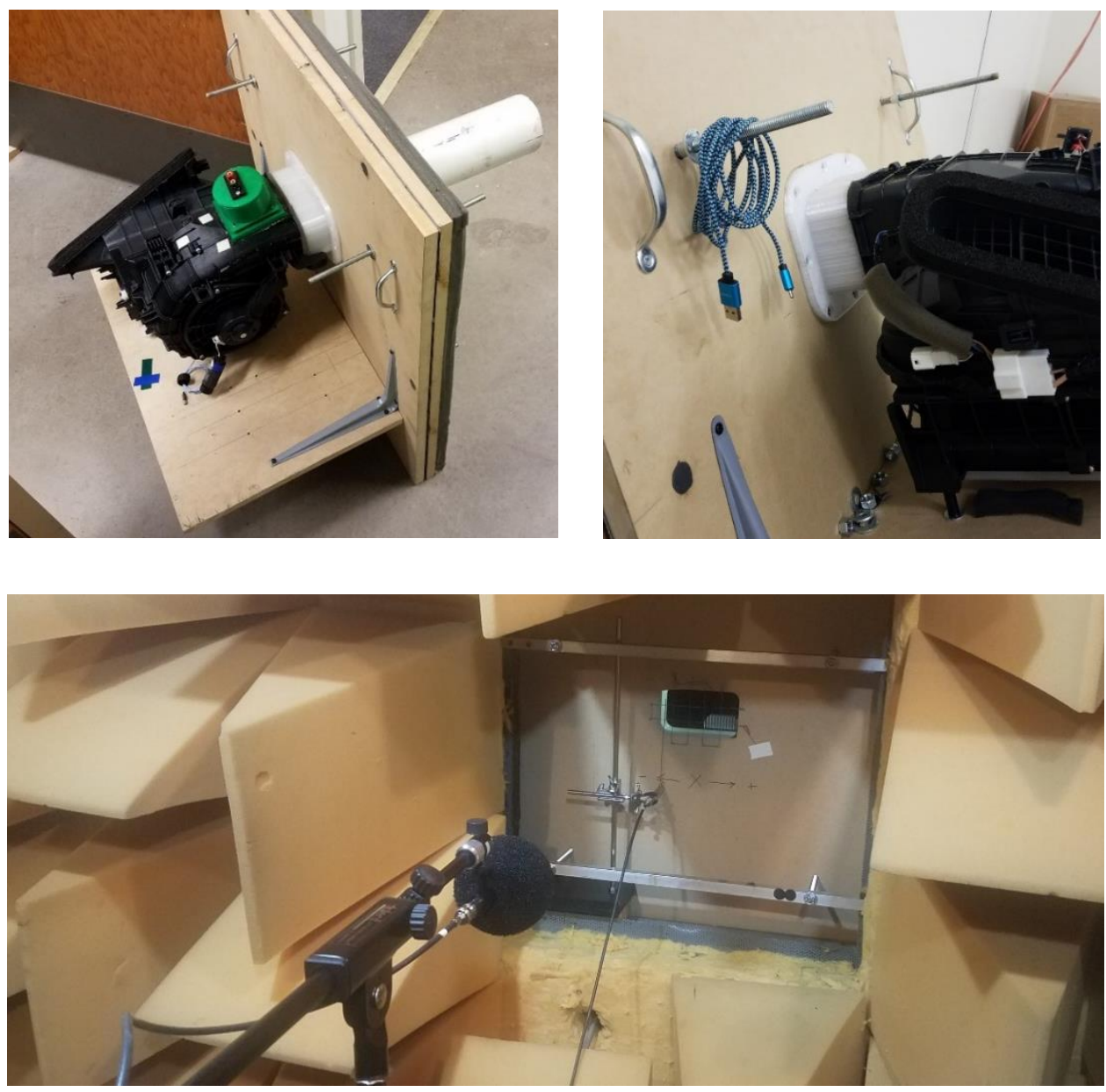

Figure 7: Installation of Blower Fan onto Partition: Fan Outlet in Anechoic Chamber

During the later phases of the case study an adapter was installed to the fan outlet so that a 4" PVC pipe could be attached to serve as a duct. Various microphone placements and disturbance sources were used across tests performed with this setup. These will be noted individually in the following experimental descriptions. The common thread across tests using this setup is that sound propagates from the fan outlet into the free-field environment of the anechoic chamber. 


\subsection{Initial Characterization of Fan Noise}

The first tests of the case study were aimed at figuring out how powerful the thermophone would need to be in order to effectively cancel fan noise in the HVAC system. Background info on ANC systems revealed that the actuator must be able to produce sound fields identical to those produced by the disturbance [30]. In addition to being able to match the acoustic field of the disturbance, this also means that the actuator should have at least an identical acoustic output level to the disturbance. To set a benchmark for this, the partitionmount was used. Initial measurements were made with a microphone placed $1 \mathrm{~m}$ from the front of the fan outlet within the anechoic chamber. It was found however, that the high airflow over the microphone caused corruption of the fan's noise spectrum. Sensor noise caused by airflow was reduced via the addition of a second microphone, placed out of the airflow (Figure 8). Using the two mic signals the coherent output power at the original microphone location was calculated, thus removing flow noise (Figure 9).

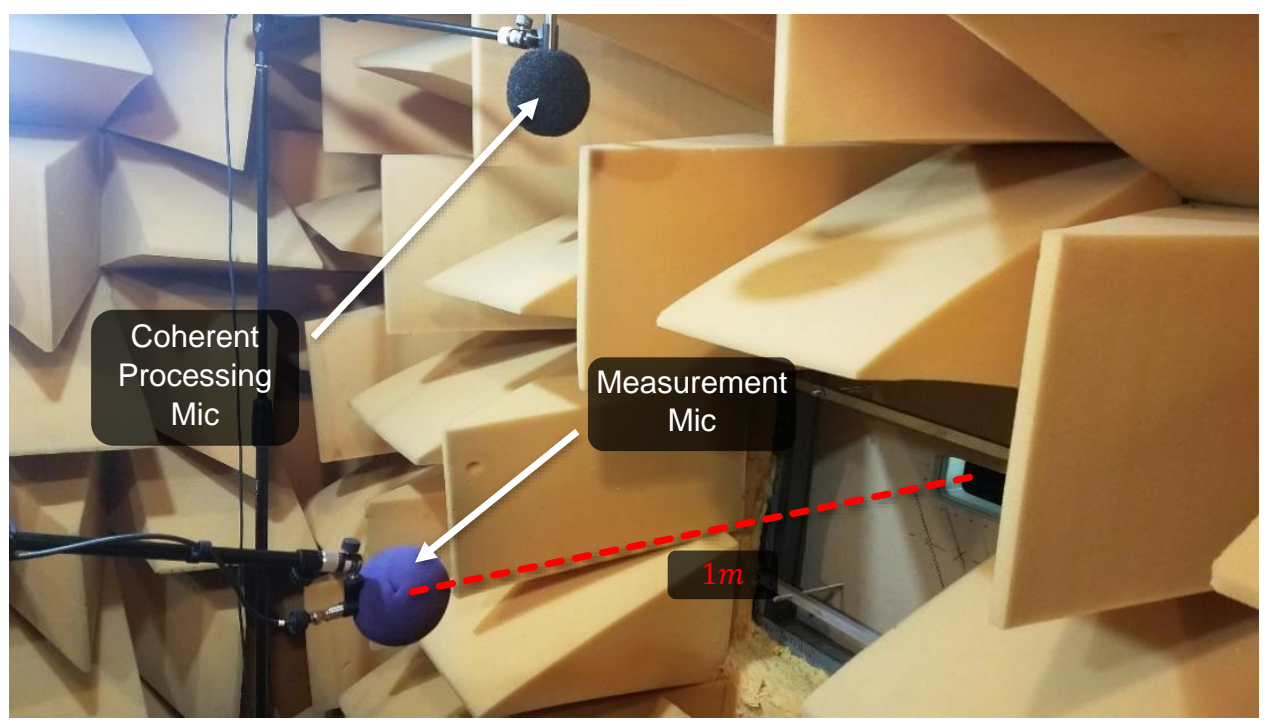

Figure 8: Microphone Locations for Initial Fan Noise Benchmark Testing 


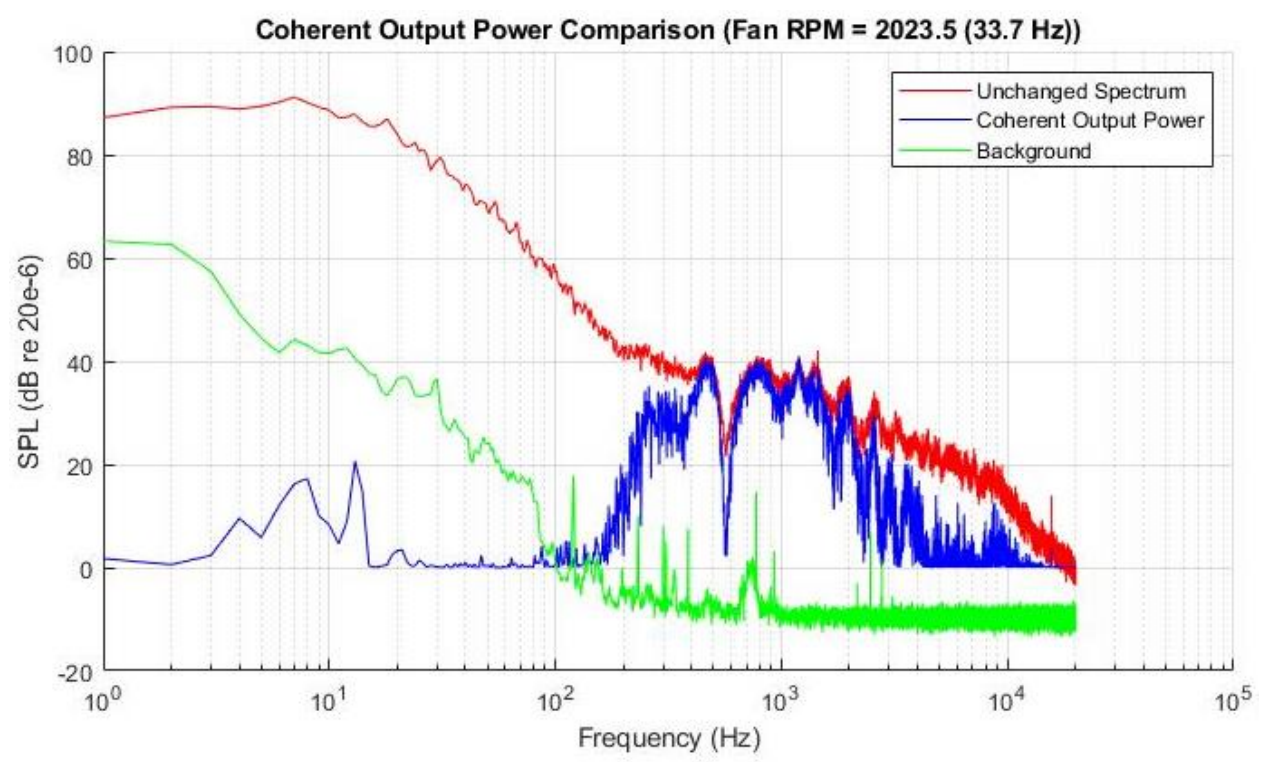

Figure 9: Initial Measurements of Fan Spectra at Measurement Mic Location

\subsection{Acoustic \& Temperature Performance Analysis of the RC01 Thermophone Prototype}

A simple acoustic test was performed to verify that the $\mathrm{RC} 01$ prototype could produce adequate acoustic output levels. Broadband noise with third-octave levels matching the blower fan noise was played through the thermophone at increasing power levels until the required overall SPL was met. To circumvent the nonlinearity of the thermophone, amplitude modulation was used to condition the signal. The partition-mount setup was used for this experimental suite, and the microphone placement was identical to placement in the initial characterization of fan noise (Figure 8). It was found that 58.8 watts of power were required for the RC01 prototype to match the overall level of the blower fan noise as computed from the coherent output spectrum at the microphone location (Figure 10). 


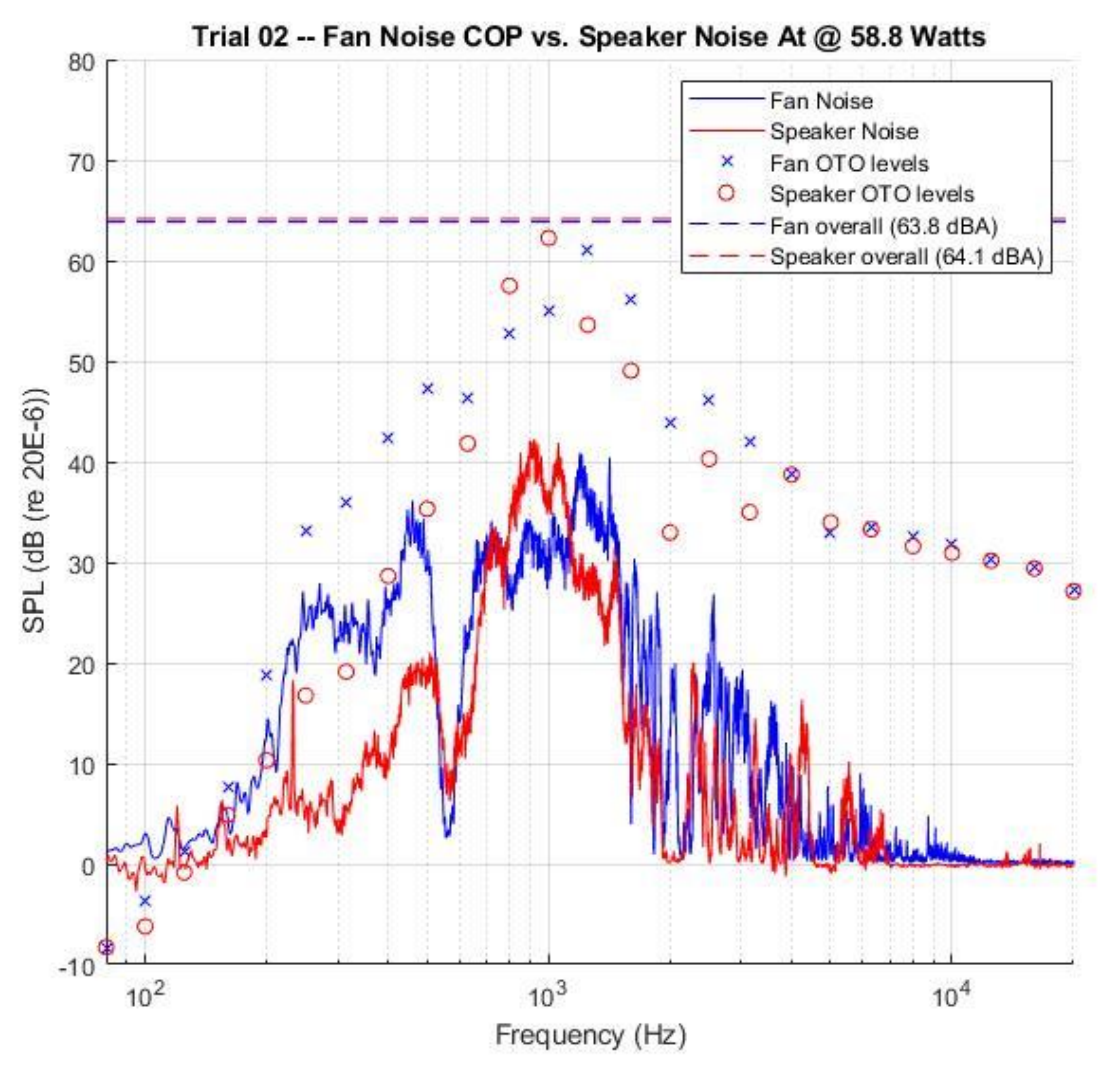

Figure 10: Comparison Between the COP Spectrum of the Blower Fan to a Broadband Output from the RC01 Thermophone

Temperature testing was also performed during this test suite. Broadband signals of varying power levels were played through the thermophone and temperatures were recorded by two thermocouples until steady state was reached (Figure 11). The blower fan was set to varying speeds across trials as well (Figure 12 \& Figure 13). 


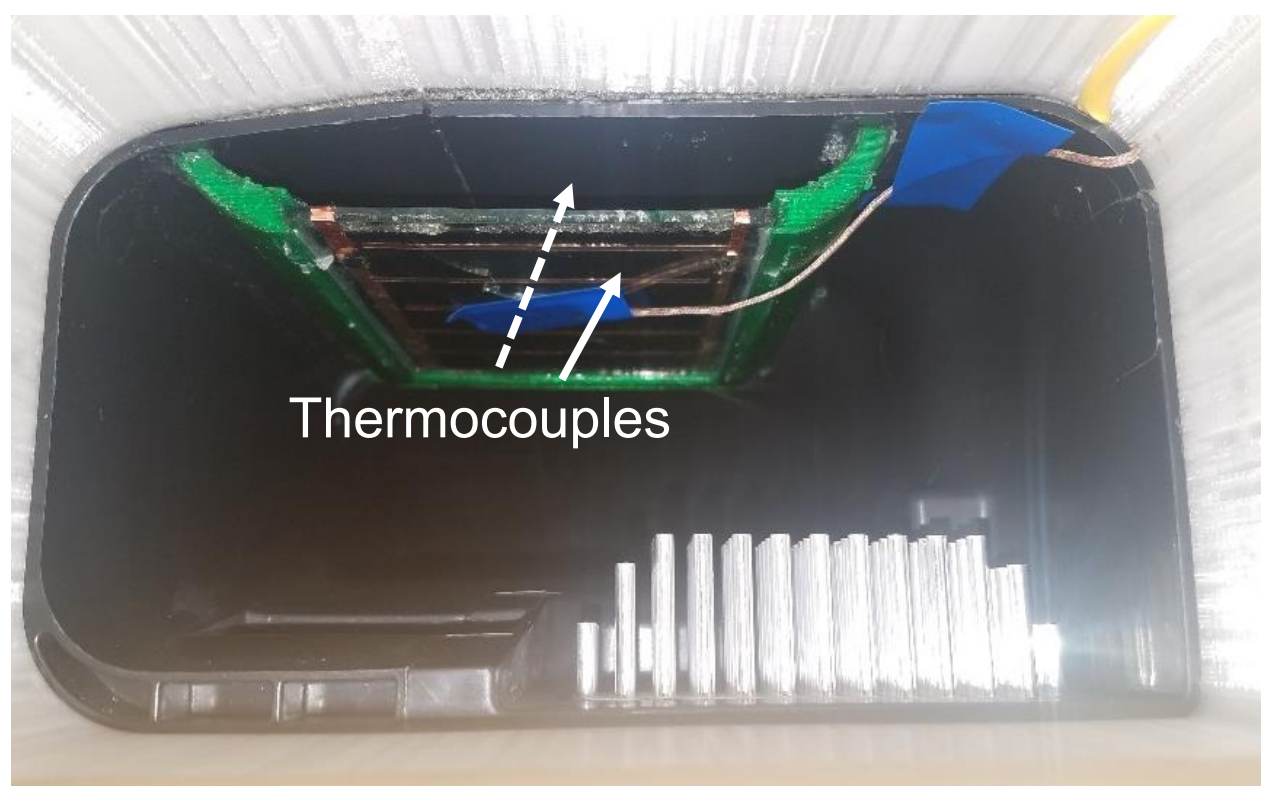

Figure 11: Thermocouple Placement For Temperature Measurements at Two Locations on the RC01 Prototype

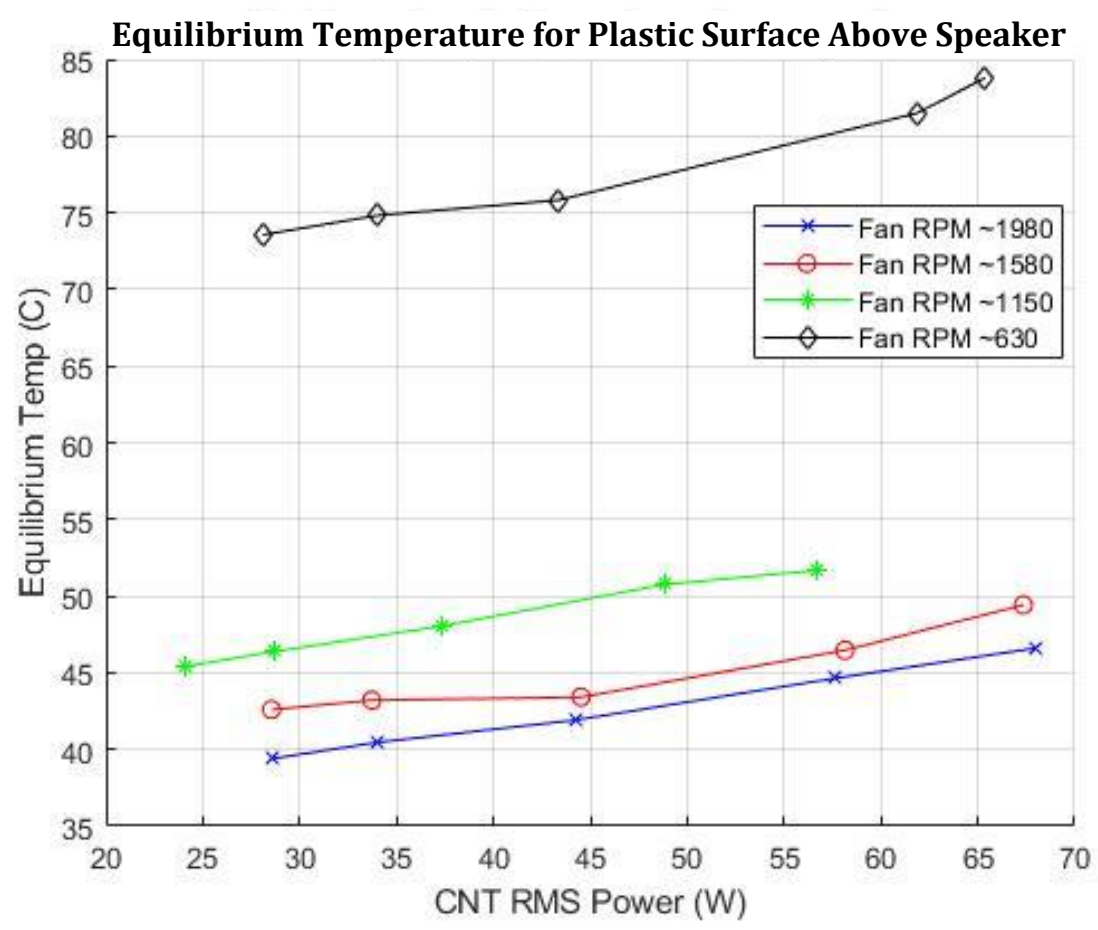

Figure 12: Steady-State Temperature of Blower Fan Plastic within CNT Chamber During Operation of RC01 Thermophone 


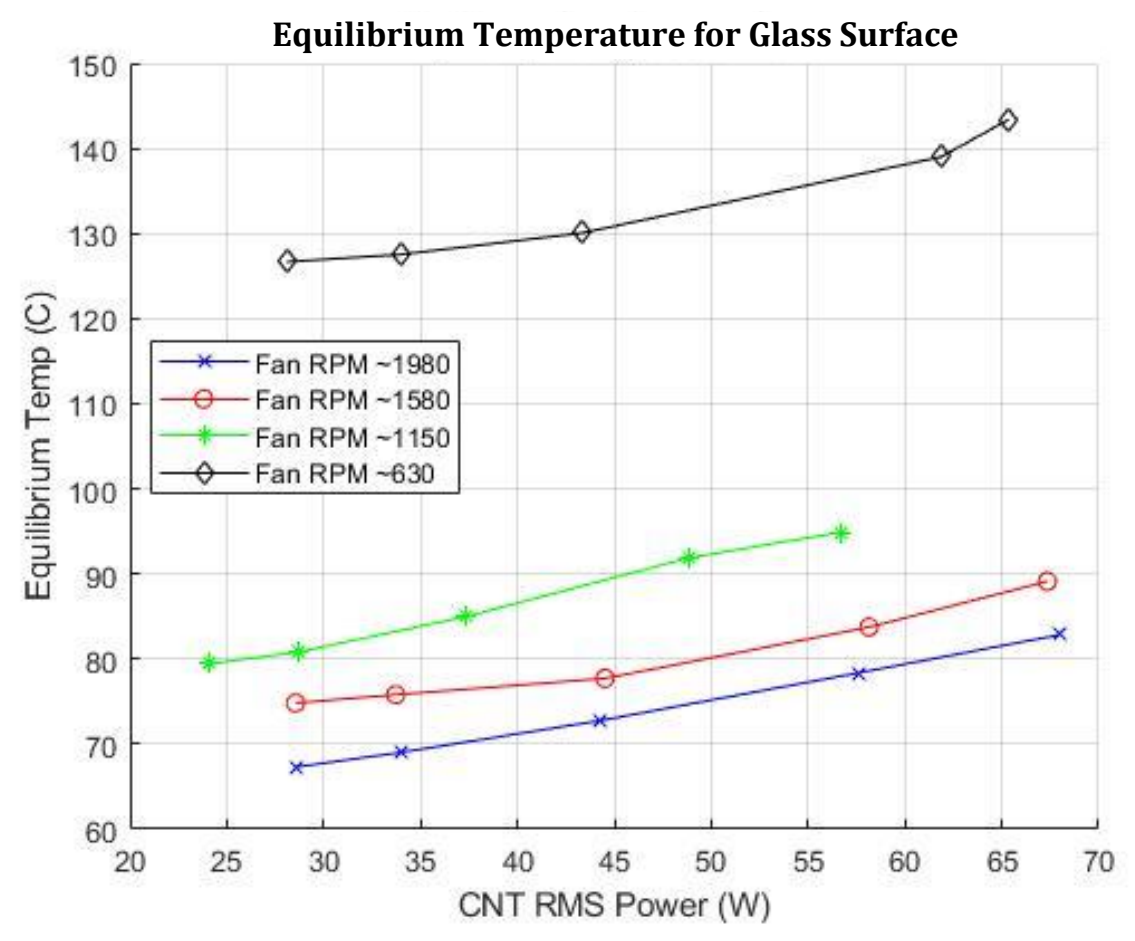

Figure 13: Steady-State Temperature of Glass Panel Outside CNT Chamber During Operation of RC01 Thermophone

\subsection{Initial Acoustic \& Temperature Performance Analysis of the RC03 Thermophone Prototype}

The second prototype ( $\mathrm{RCO3})$ to be installed into the blower fan also faced a series of tests meant to characterize it's performance. Since the RC03 prototype altered the actual geometry of the blower fan, the scope of this testing went beyond the RC01 suites and included investigations into how these fundamental geometry changes affected the noise of the fan. The full HVAC setup was used for these tests; a $1 / 2$ " free-field microphone set at the driver's ear location was used for measurements (Figure 14). 


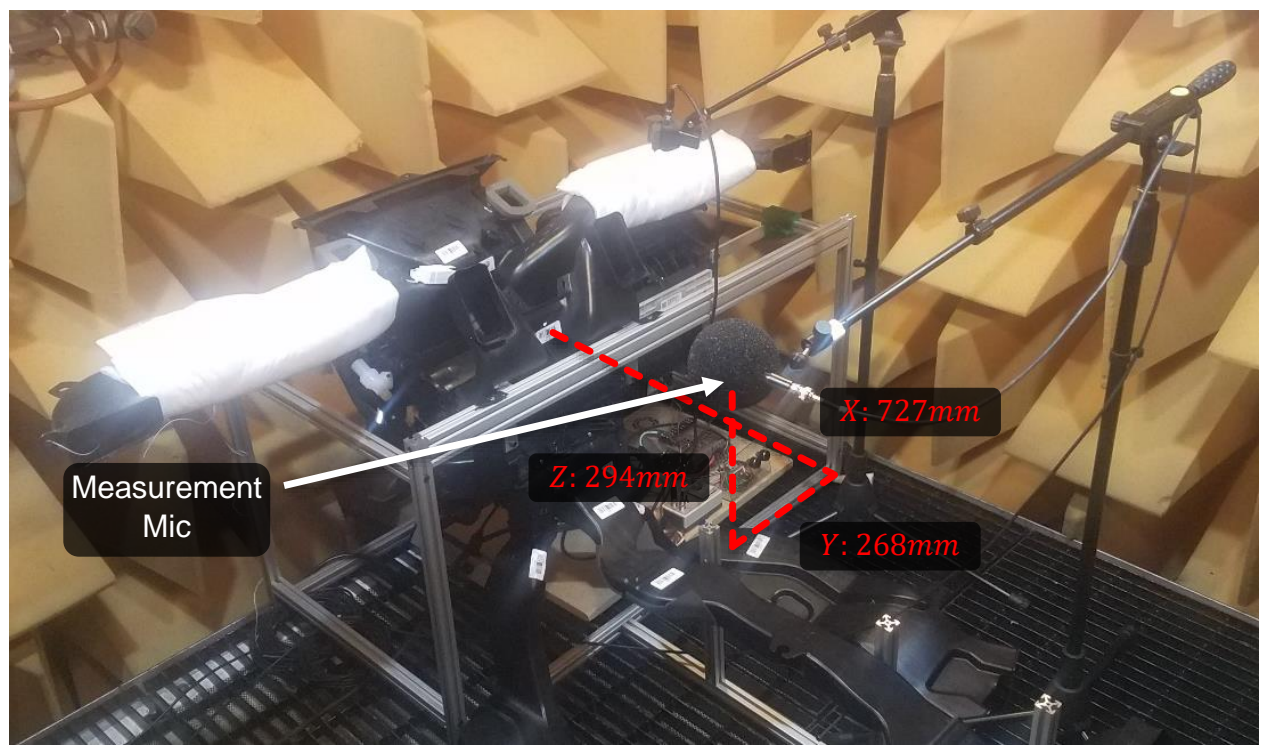

Figure 14: Microphone Placement for Measuring the HVAC Noise Spectra and Overall Acoustic Output Level of the RC03 Prototype in the Full HVAC Setup

Of all the geometry changes made to the blower fan for this prototype, the most significant was the addition of an "acoustic slot" connecting the inside of the fan's exhaust to the CNT chamber of the thermophone (Figure 15).

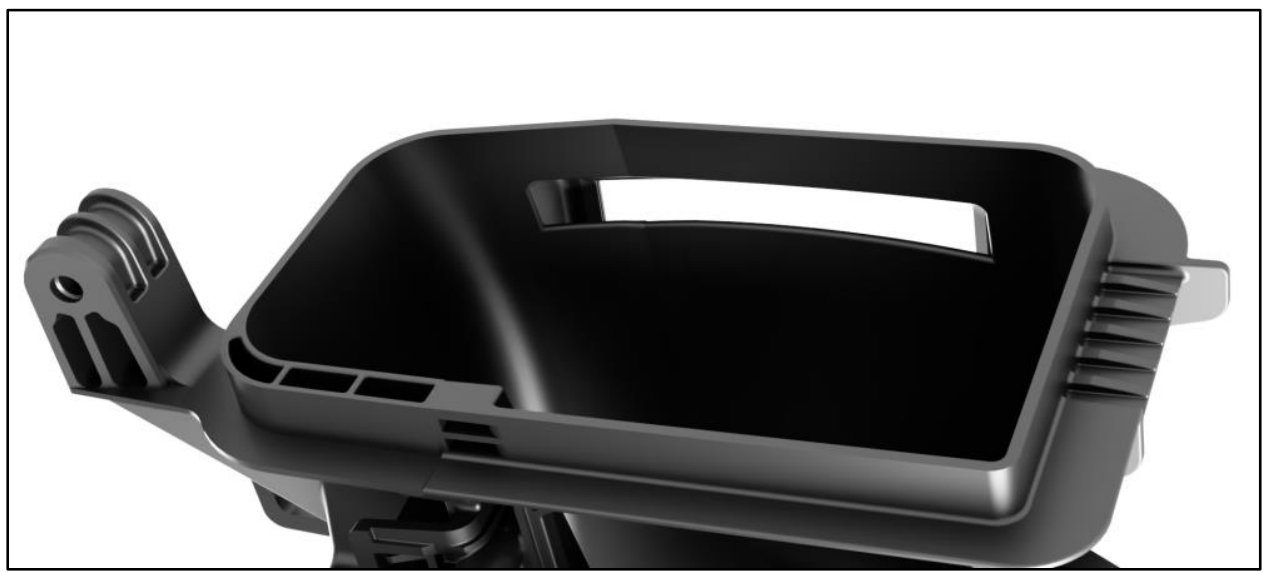

Figure 15: Acoustic Slot Required for Propagation of Sound into the HVAC from the CNT Chamber of the RC03 Thermophone Prototype

This slot was identified as a potential source of whistling by the project sponsor's engineering team. To ensure that the slot was not another source of noise during fan operation, acoustic measurements were taken of both the original and modified fan geometries during operation in the full HVAC setup (Figure 16). The RC01 prototype was removed from the original blower fan for this experiment; this served as the control. 


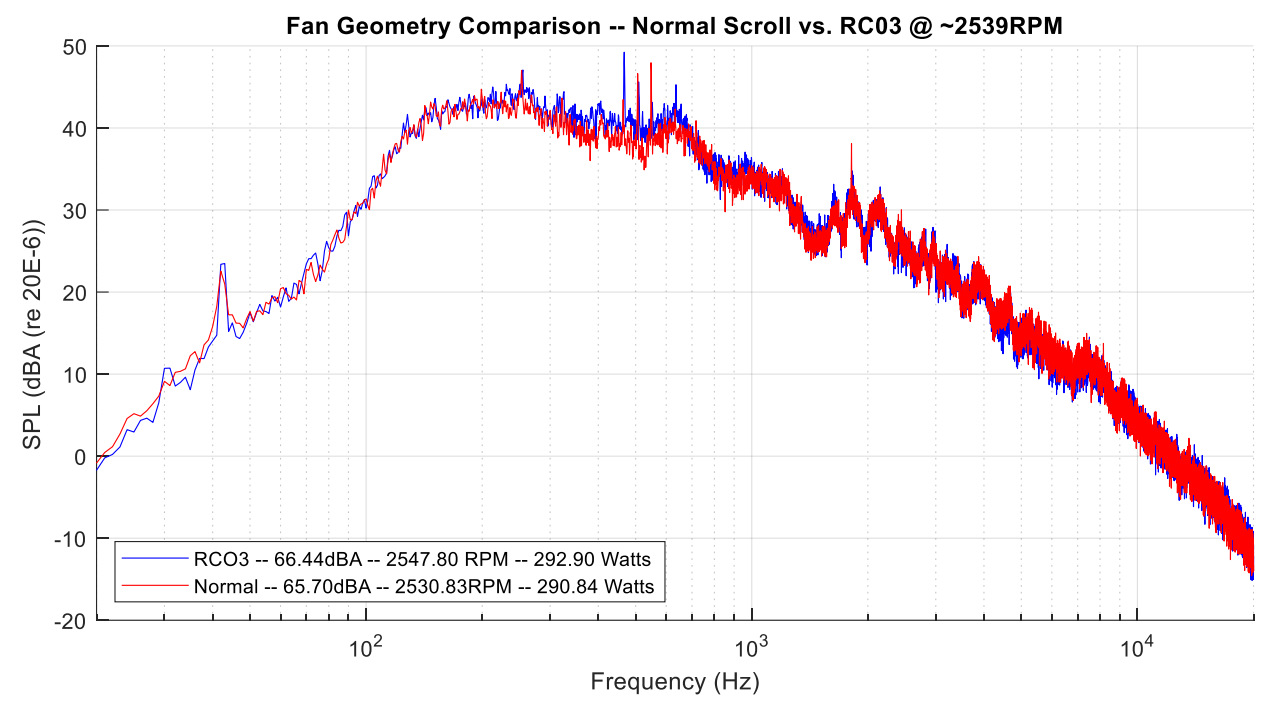

Figure 16: Comparison of HVAC Noise Spectra With Two Fans Geometries Installed - RC03 Prototype \& Nominal Fan Geometry

After determining that RC03's acoustic slot was not significantly altering the HVAC noise, trials to measure the performance of the RC03 thermophone began. Broadband noise with third octave levels identical to the blower fan spectrum (in the full HVAC setup) was created and used as the output of the thermophone. Output spectra were recorded at the driver's ear location while the thermophone power was increased incrementally until the thermophone's maximum power ${ }^{1}$ was reached (Figure 17).

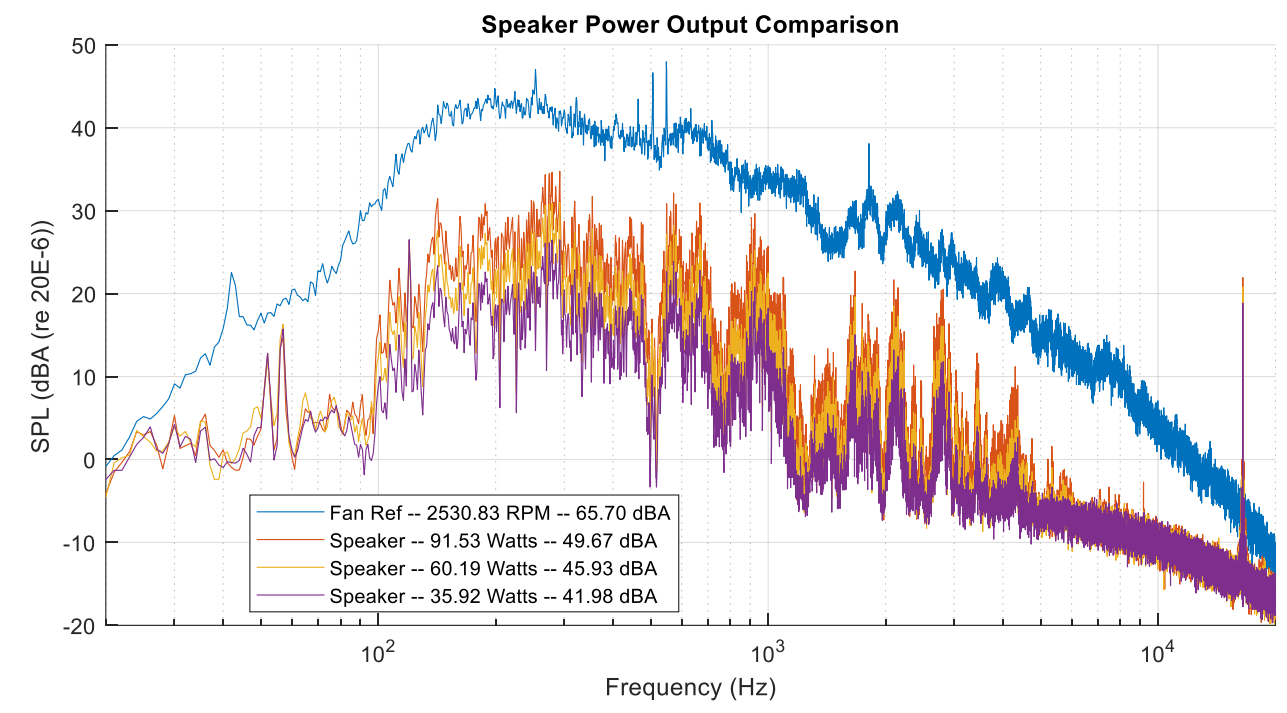

Figure 17: Comparison of HVAC Noise Spectra to the Broadband Output of the RC03 Prototype at Varying Power Levels

${ }^{1}$ Power input at which the CNT reaches full power density, around $2 \frac{\mathrm{W}}{\mathrm{cm}^{2}}$ for a 5 -layer thermophone. 
It was observed that increasing the power beyond $\sim 90 \mathrm{~W}$ was starting to introduce melting to plastic regions of the thermophone and fan geometry. Power levels were not increased enough to allow RC03's output spectra to match the fan spectra.

After viewing the results of the acoustic testing, it became clear that the power input to the RC03 thermophone would need to be large during cancellation trials. Thermal testing for this speaker seemed very important in this context. Reinforcing this importance were the poor heat transfer characteristics of the RC03 design. The CNT panel was moved away from the flowing air inside the fan exhaust outlet, so less cooling would occur, and it was theorized that the plastic surfaces making up the CNT chamber would get much hotter than in the RC01 prototype. Three thermocouples were installed onto the thermophone to explore this (Figure 18).
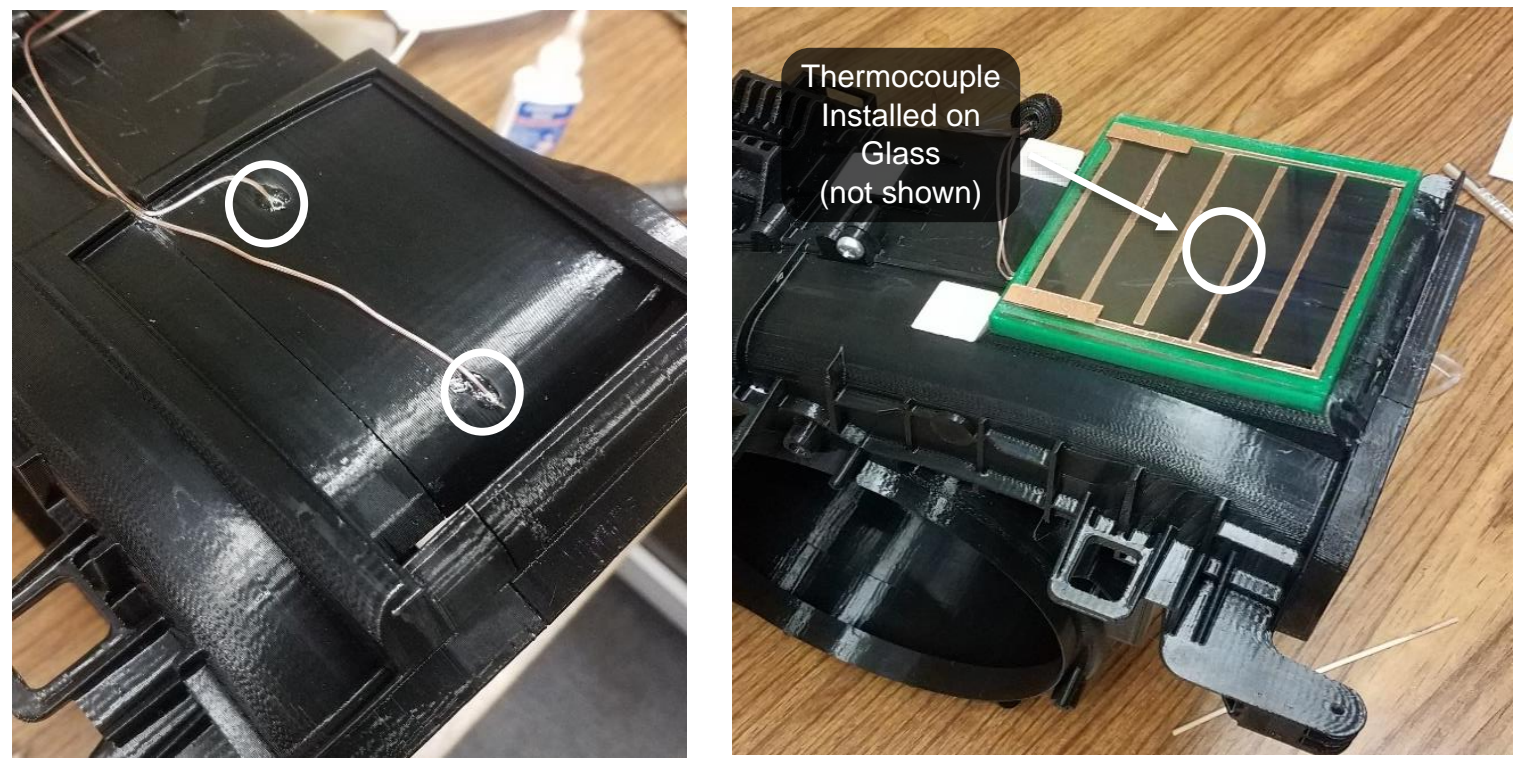

Figure 18: Highlighted Thermocouple Placements for RC03 Thermal Testing

Broadband signals at multiple power levels were input to the speaker and temperature readings were taken for 300 seconds. Measurements for each power level were taken twice; once with the fan completely off, and once with the fan spinning at $~ 2500 \mathrm{rpm}$. Readings from the thermocouple nearest the acoustic slot (middle thermocouple, Figure 18) were not reported since they were consistently lower than readings from the other thermocouple in the CNT chamber. Results of testing show that the RC03 prototype gets hotter than RC01 during operation (Figure 19). 

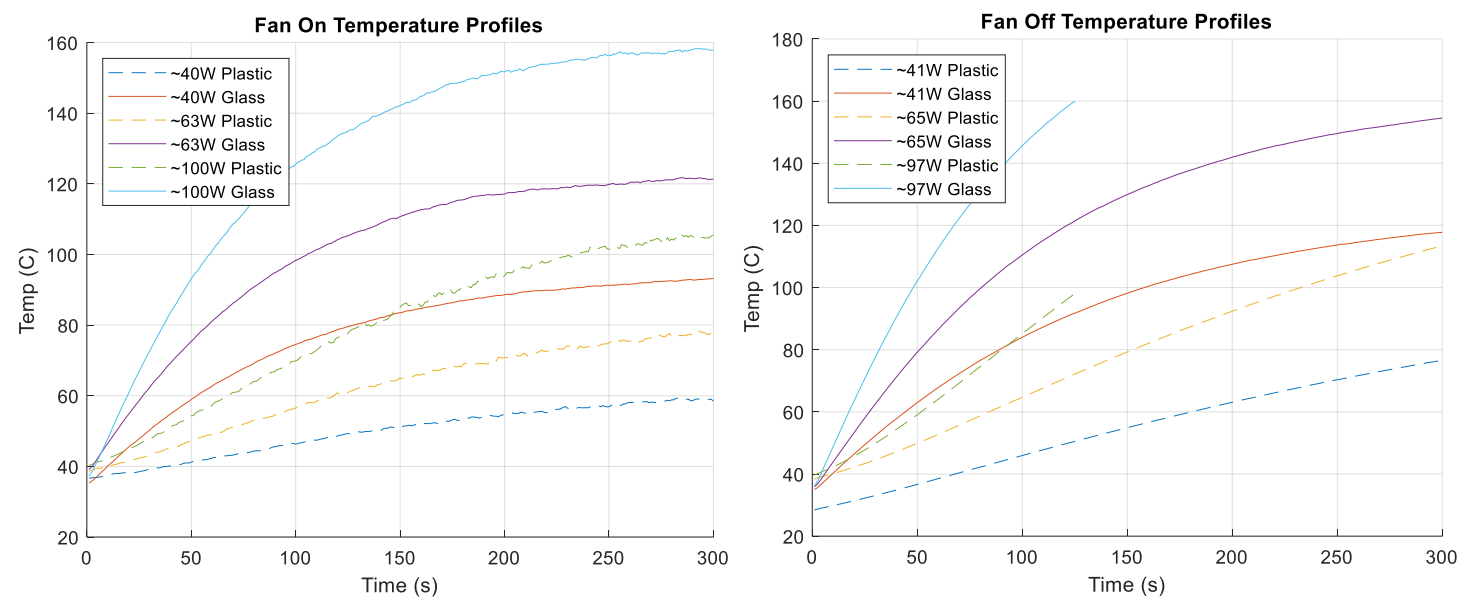

Figure 19: Temperature at two Points on the RC03 Prototype Over Time - Varying Power Levels and Fan Speeds

\subsection{GLSV ANC Controller Performance Testing \& ANC Demo}

It was seen from the last test suite that in the HVAC setup, the RC03 prototype was not able to match the overall levels produced by the blower fan at the driver's ear location. Despite this, it was still used alongside the GLSV ANC Controller in the case study's initial ANC trials. This decision resulted from the fact that the controller uses a tonal cancellation algorithm; this type of cancellation only requires that the actuator's output match the disturbance level at one tone.

The full HVAC setup was used for this experiment; one of the primary goals was to evaluate the overall effectiveness of tonal cancellation in the system. The secondary goal was to provide a technology demo for the project sponsor's engineering team. A laser tachymeter was used to provide a reference signal to the GLSV controller. Also serving as an input to the controller (error signal) was a $1 / 2$ " free-field microphone, located $1 \mathrm{~m}$ from the HVAC center at the standard driver's ear location (Figure 20). 


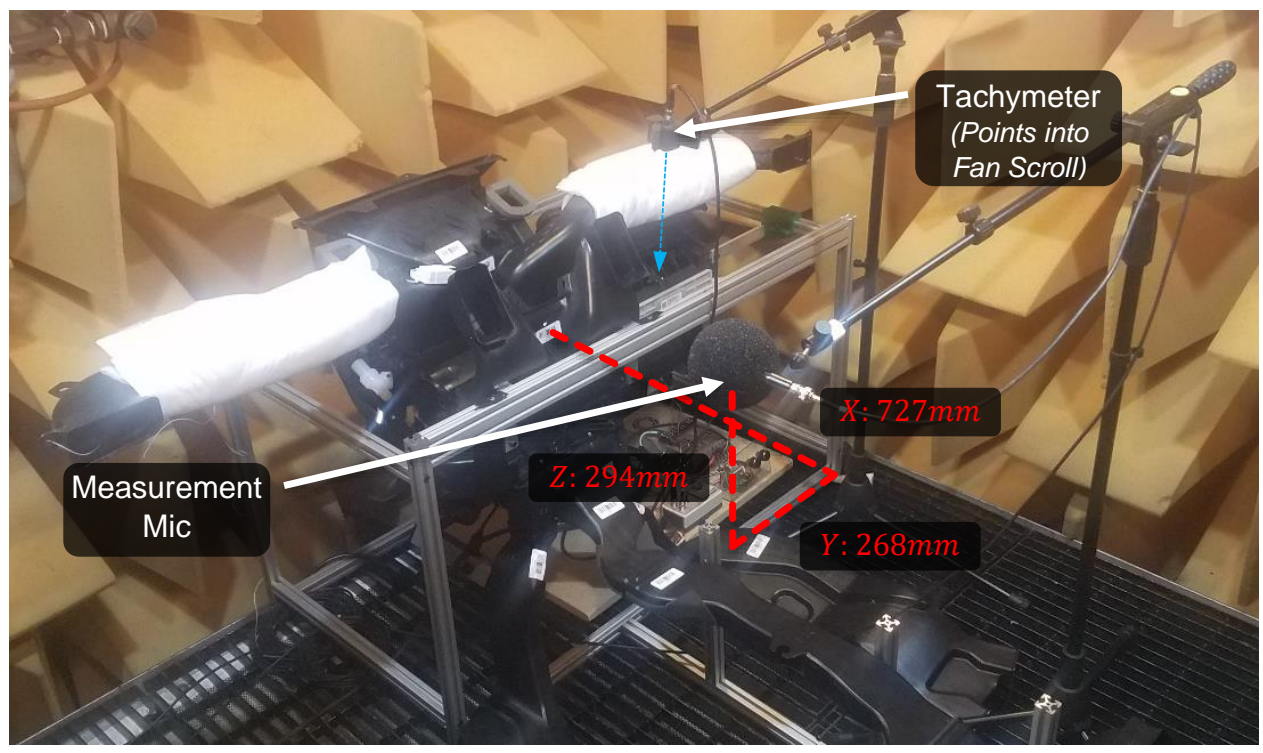

Figure 20: Full HVAC Configuration with Free Field Measurement Microphone \& Tachymeter Positioned for Tonal Cancellation Trials

All spectra reported for this experiment were taken using this microphone. An external amplitude modulator provided signal conditioning for the thermophone. After looking at the fan spectrums from the first RC03 testing suite, the $11^{\text {th }}$ and $13^{\text {th }}$ orders were chosen as cancellation targets since their elimination had the highest potential to impact overall sound levels of the HVAC (Figure 21).

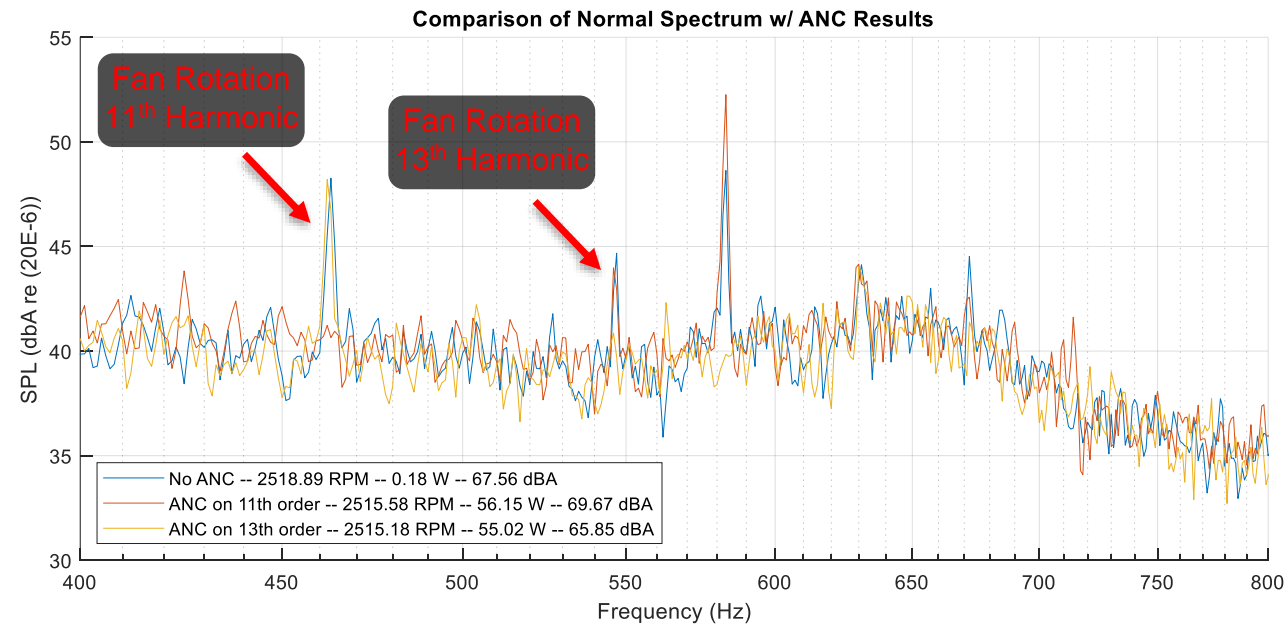

Figure 21: Comparison of Nominal HVAC Noise Spectra to Two Spectra Experiencing Tonal Cancellation

As can be seen in the plot above, spectra, overall SPLs, fan RPM, and speaker power were all recorded for trials throughout this experiment. While the GLSV controller was able to reduce the $11^{\text {th }}$ and $13^{\text {th }}$ order tones to the noise floor of the broadband spectra, this ultimately had little effect on the overall levels. Subjectively, a change in fan noise after the application of ANC at either order was not noticeable. 
Another odd result which should be mentioned is the presence of an intermittent tone across the three spectra at $\sim 585 \mathrm{~Hz}$. Despite only showing up in two trials, this tone was not subject to any cancellation. It was found to be intermittent across other fan noise recordings in the full HVAC setup. Interestingly enough, the presence/absence of this tone has more effect on the overall fan levels than the cancellation of the $11^{\text {th }}$ or $13^{\text {th }}$ order tones. This is the cause for overall level of the $11^{\text {th }}$ order cancellation actually being higher than the "no ANC" case.

\subsection{Temperature Testing of the Kapton Encapsulated Flexible Thermophone Design}

As concerns about the manufacturability and sound output of rigid thermophones continued to mount, more effort was focused on the testing and development of flexible thermophones. One such test was a simple proof of concept to check whether mounting thermophones directly on the plastic interior surfaces of the fan was feasible. The main concern surrounding this configuration was exposure of the plastic to the high temperatures present at the CNT surface. A small four-layer composite thermophone was constructed using Kapton film as the encapsulation material. The thermophone was then mounted near the exhaust outlet of the blower fan with a thermocouple underneath (Figure 22).
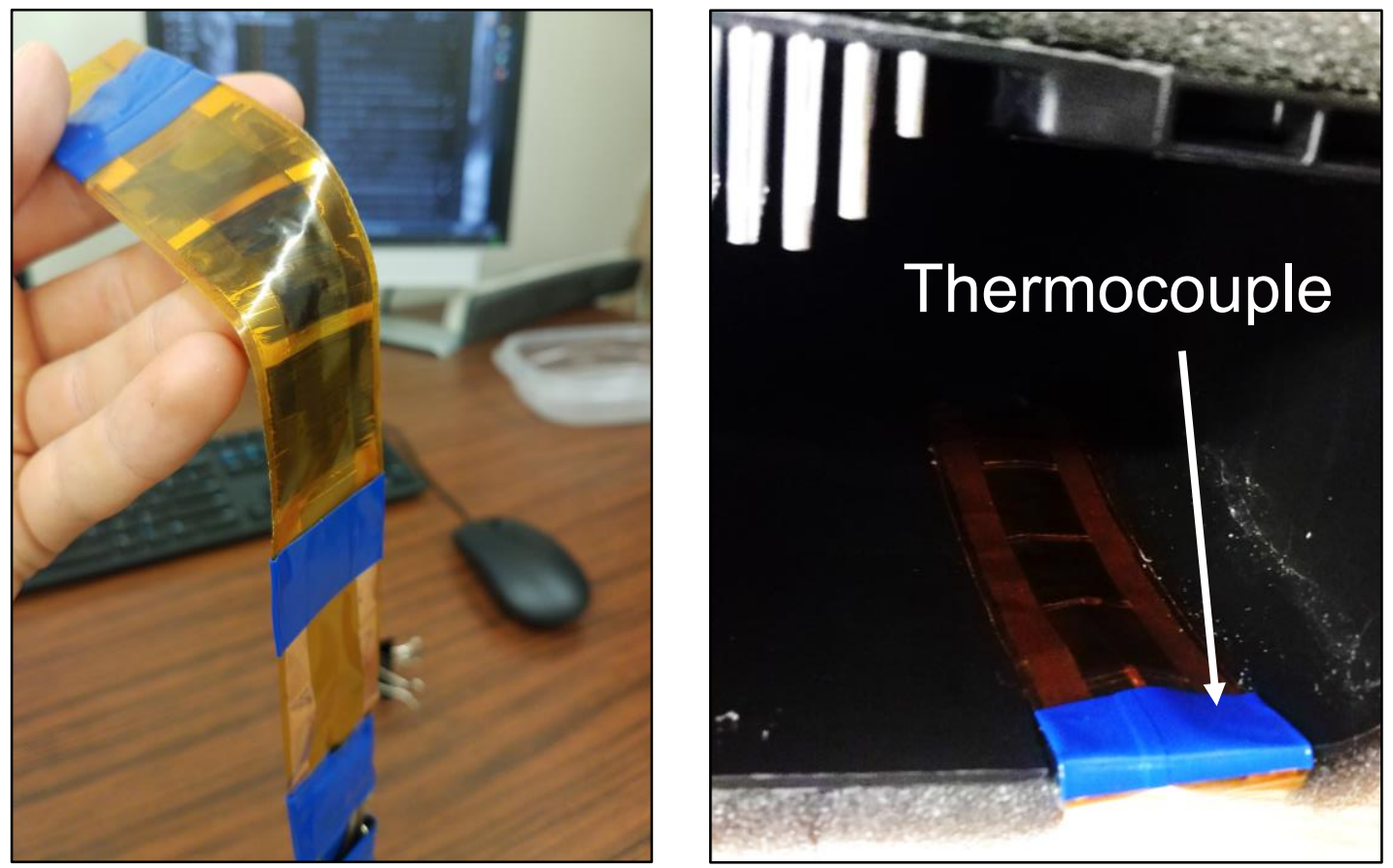

Figure 22: Kapton Strip Flexible Thermophone Before and After Installation into Blower Fan

In order to make the tests more scalable, the power density of the speaker was recorded in lieu of the actual power delivered to the CNT. Broadband signals of increasing power were played through the thermophone once it was installed in the blower fan. The 
temperature was then measured over time for a variety of fan speeds at the different CNT power densities (Figure 23).

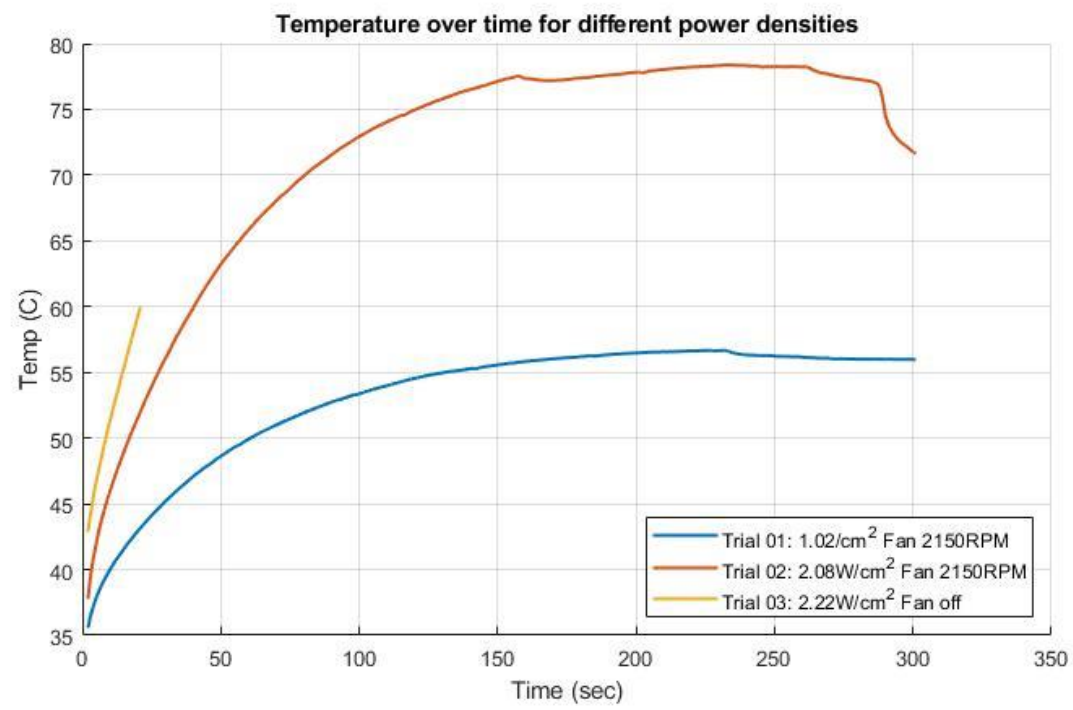

Figure 23: Temperature of Fan Surface Under Kapton Strip Flexible Thermophone During Fan/Thermophone Operation

\subsection{Testing to Identify the Ideal Material for Flexible Thermophone Encapsulation}

Initial temperature testing showed that mounting a flexible thermophone directly to the inner surfaces of the HVAC could be a legitimate design option. This, along with the versatility-derived advantages offered by flexibles, prompted a more rigorous design study to be performed on the four-layer composite design. A group of candidate film materials (Table 1) that could potentially make good encapsulation layers was selected. Acoustic and thermal properties of these films were investigated. To characterize them acoustically, classic transmission loss (TL) and insertion loss (IL) measurements were taken. Additionally, a special case of IL where the film directly touches the CNT film was measured. Thermal properties were judged on a pass/fail basis by constructing speakers using the materials and monitoring damage during operation.

Transmission loss measurements were made using four $1 / 4$ " array microphones inside a Brüel and Kjær Type 4206 impedance tube (Figure 24). Measurement procedures from ASTM E2611-19 were followed [35]. Three samples were tested for each candidate material, and the average transmission loss across samples was calculated (Table 1). 


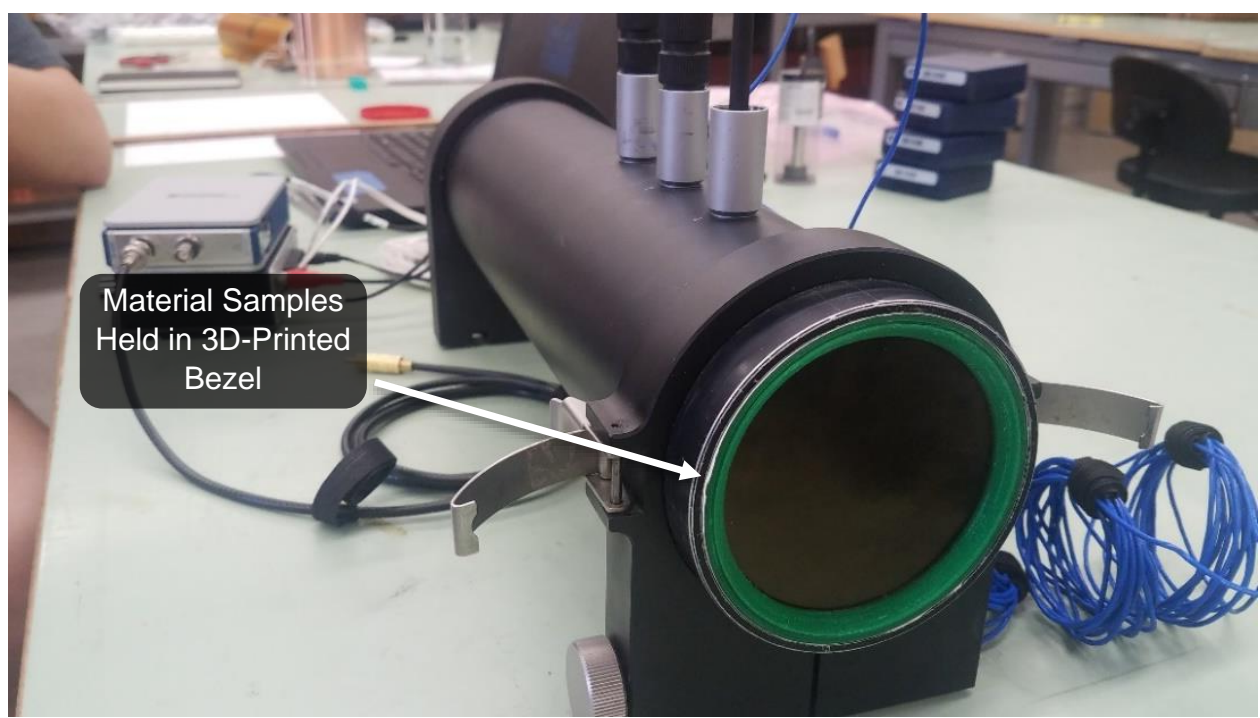

Figure 24: Impedance Tube Used for Encapsulation Material Transmission Loss Testing

Table 1: Transmission Loss of CNT Encapsulation Candidate Materials

\begin{tabular}{|c|c|}
\hline Material & $\begin{array}{c}\text { Transmission } \\
\text { Loss } \\
\text { Average (dB) }\end{array}$ \\
\hline Kapton $\frac{1}{3}$ Mil & 2.377 \\
\hline Kapton 1 Mil & 3.923 \\
\hline PEN 2 Mil & 2.812 \\
\hline PFA 1 Mil & 2.931 \\
\hline Fiberglass Weave & 0.299 \\
\hline
\end{tabular}

In addition to TL measurements on the candidate encapsulation materials, IL measurements were also made. This was done using part of the impedance tube (Figure 24 ) in the anechoic chamber. The impedance tube was left open, white noise was played through its speaker, and an overall SPL measurement was taken using a microphone near the tube exit. Material samples were then placed in the opening of the impedance tube and another measurement of the white noise level was taken. The difference between these two numbers was used as the IL (Table 7).

Table 2: Insertion Loss of CNT Encapsulation Candidate Materials

\begin{tabular}{|c|c|}
\hline Material & $\begin{array}{c}\text { Insertion Loss } \\
\text { Average (dB) }\end{array}$ \\
\hline Kapton $\frac{1}{3}$ Mil & 3.3 \\
\hline Kapton 1 Mil & 5.8 \\
\hline PEN 2 Mil & 7.7 \\
\hline PFA 1 Mil & 6.6 \\
\hline Fiberglass Weave & 1.6 \\
\hline
\end{tabular}


It was also theorized that attenuation due to stretching a film directly over a CNT thermophone could not be accurately predicted using IL alone. This is because of the thermoacoustic effect. A thermophone needs to change the temperature of the air around it to create sound; directly touching the CNT with a film could affect its ability to do this. To investigate this, a second round of modified IL testing was done. A rigid CNT panel was built using a ceramic-coated sheet as a substrate. The panel was then placed in the anechoic chamber with a microphone positioned above it (Figure 25).

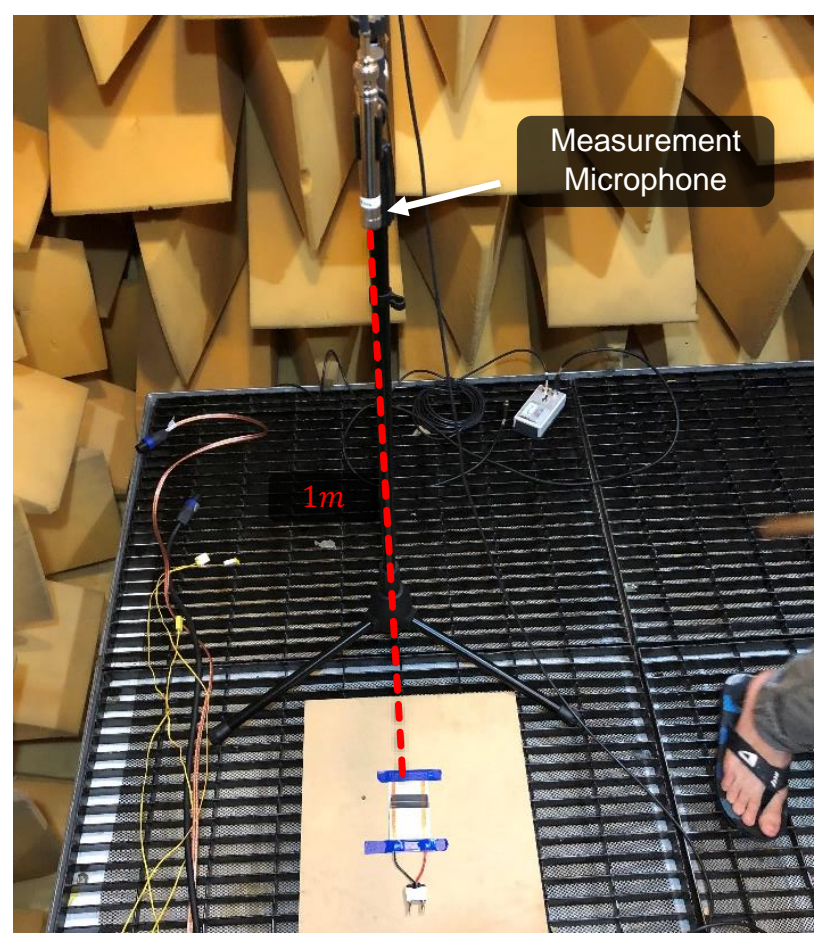

Figure 25: Setup for Measuring Output Level of Rigid Panel Thermophone for Pseudo-IL Testing

White noise was played through this speaker and the overall SPL was recorded. A candidate encapsulation film was stretched tightly over the CNT panel (Figure 26), and the volume of white noise played through the thermophone was measured again. Once measurements were taken the panel was cleaned and new CNT was applied for the next candidate material. 

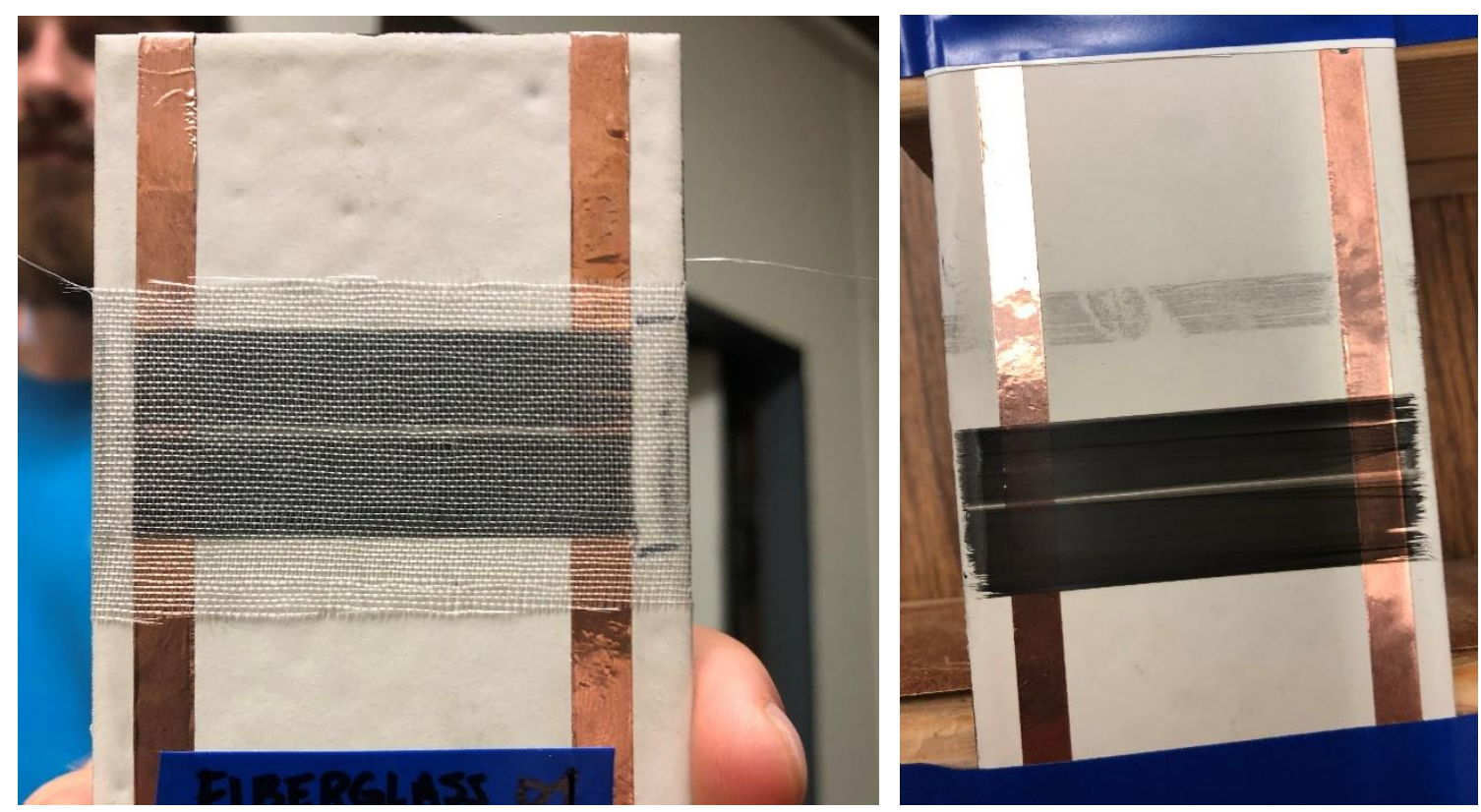

Figure 26: Rigid CNT Panel with and without Fiberglass Covering Installed

Performing this test twice for each sample showed high variation across test trials (Table 3).

Table 3: Film-on-CNT "Insertion Loss" Testing Results

\begin{tabular}{|c|c|c|}
\hline Material & $\begin{array}{c}\text { Insertion Loss } \\
\text { Trial 01 (dB) }\end{array}$ & $\begin{array}{c}\text { Insertion Loss } \\
\text { Trial 02 (dB) }\end{array}$ \\
\hline Kapton $\frac{1}{3}$ Mil & 14.4 & 7.1 \\
\hline Kapton 1 Mil & 11.1 & 13.3 \\
\hline PEN 2 Mil & 10.6 & 10.2 \\
\hline PFA 1 Mil & 16.6 & 19.2 \\
\hline Fiberglass Weave & 13.6 & 2.6 \\
\hline
\end{tabular}

\subsubsection{Acoustic and Temperature Testing for Fiberglass Encapsulated Flexible Thermophones}

Despite large variation across "film-on-CNT" IL test trials from the prior experiment, observations still pointed towards fiberglass weave to be the most effective encapsulation material tested. More experimentation was done using the fiberglass. There were three main goals behind this test suite: to get more repeatable results for the "film-on-CNT" tests, to explore the maximum power density of the four-layer composite thermophone when fiberglass is used as the encapsulation material, and to check thermal performance of the fiberglass flexibles when installed in the fan. 
For the first testing goal, two thermophones were built. A rigid CNT panel was made using glass as the substrate Then, a four-layer composite thermophone with identical CNT area and electrode configuration was built using fiberglass as the encapsulation material. The output of the rigid CNT panel was compared with the output of the flexible thermophone in two configurations. In both configurations the flexible thermophone was tightly stretched across a rigid panel, glass in one case, and ABS plastic in the other (Figure 27). These thermophones were placed in the anechoic chamber independently, at identical locations relative to a $1 / 2$ " free-field microphone (Figure 25). Band-limited white noise $(200-2000 \mathrm{~Hz})$ was played through the thermophones \& two acoustic measurements were made for all three thermophone configurations (Figure 28). The two measurement spectra (per configuration) were then averaged together, and an overall level within the 200 to $2000 \mathrm{~Hz}$ band was calculated (Table 4).
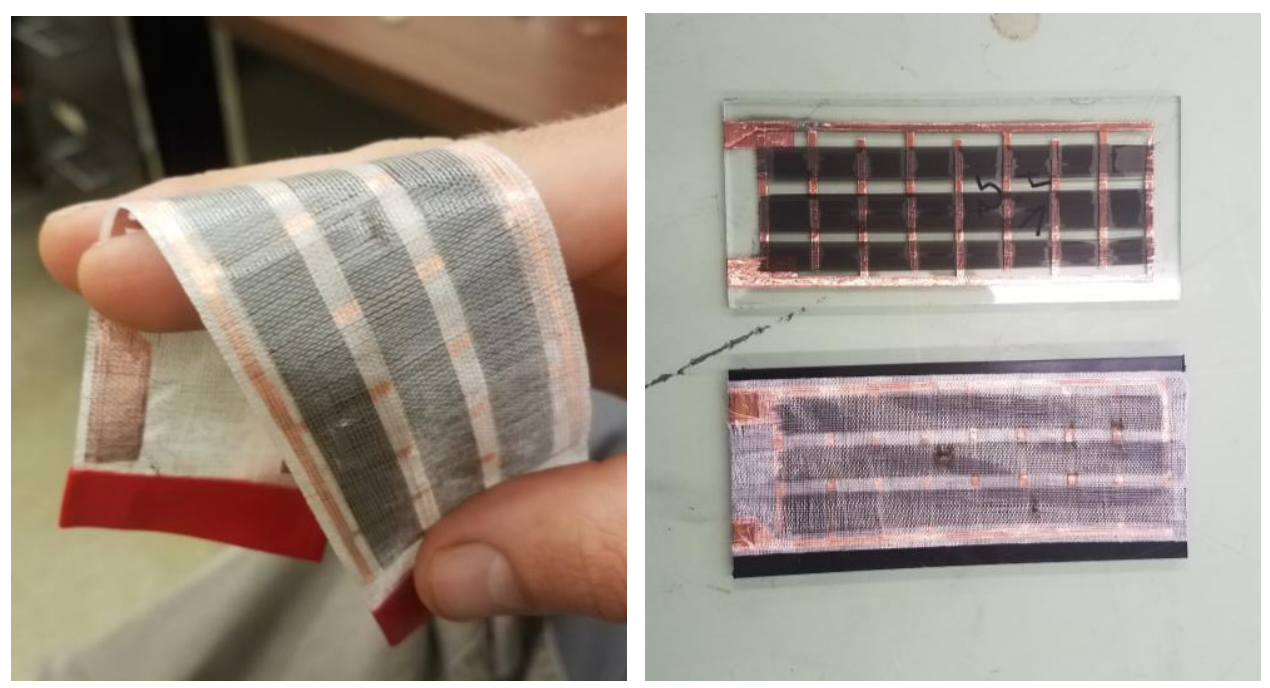

Figure 27: Flexible Thermophone - Rigid CNT Panel \& Flexible Thermophone Installed on ABS Panel 


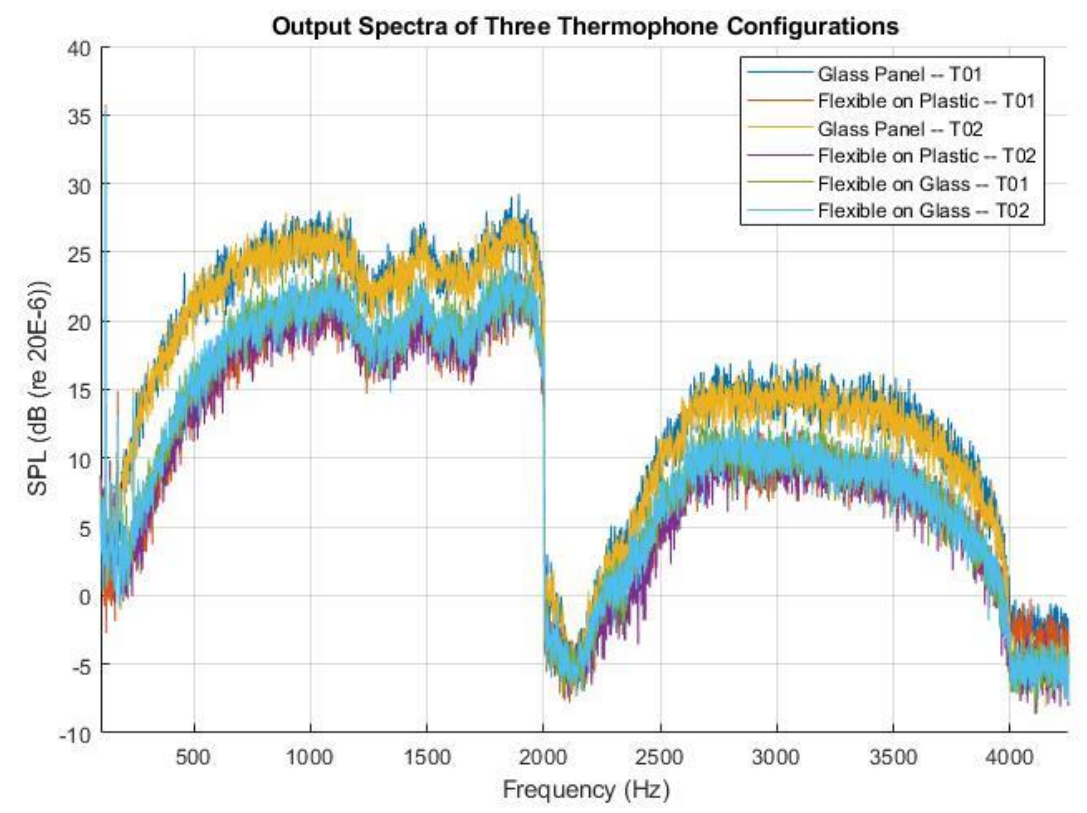

Figure 28: Comparison of Glass Panel, Flexible on Glass, and Flexible on Plastic Thermophone Output Spectra

Table 4: SPL within the $200-2000 \mathrm{~Hz}$ Band for Three Different Thermophone Configurations

\begin{tabular}{|c|c|}
\hline Thermophone & $\begin{array}{c}\text { RMS SPL } \\
(\mathbf{2 0 0}-\mathbf{2 0 0 0 H z}) \mathbf{~ d B}\end{array}$ \\
\hline Rigid Panel on Glass & 23.2 \\
\hline Flexible on Plastic & 17.8 \\
\hline Flexible on Glass & 18.7 \\
\hline
\end{tabular}

To investigate the power density limitations of the fiberglass weave encapsulation material, simple subjective tests were performed. A flexible fiberglass thermophone with a CNT area of $\sim 300 \mathrm{~cm}^{2}$ was constructed. The thermophone was then hung from a microphone stand, and signals of increasing power were played through it. Power was increased from $120 \mathrm{~W}$ until visible degradation of the thermophone occurred. 
Table 5: Behavior of Flexible Thermophone at Different Power Densities

\begin{tabular}{|c|c|c|}
\hline $\begin{array}{c}\text { Electrical Power } \\
(\boldsymbol{W})\end{array}$ & $\begin{array}{c}\text { Thermophone Power } \\
\text { Density } \\
\left(\frac{\boldsymbol{W}}{\mathbf{c m}^{2}}\right)\end{array}$ & $\begin{array}{c}\text { Thermophone } \\
\text { Behavior }\end{array}$ \\
\hline 120 & 0.4 & Nominal \\
\hline 210 & 0.7 & Nominal \\
\hline 300 & 1.0 & Nominal \\
\hline 411 & 1.4 & Nominal \\
\hline 500 & 1.7 & $\begin{array}{c}\text { Started to Smoke and } \\
\text { Emit Chemical Odor }\end{array}$ \\
\hline 470 & 1.6 & Nominal \\
\hline
\end{tabular}

Fiberglass thermophones were also subject to temperature testing like what was done for the Kapton strip thermophone. When the FC01 prototype was installed in the blower fan, a thermocouple was placed between it and the plastic of the fan (Figure 29). Signals of varying power were played through the thermophone and 300 seconds of temperature data was recorded (Figure 30).

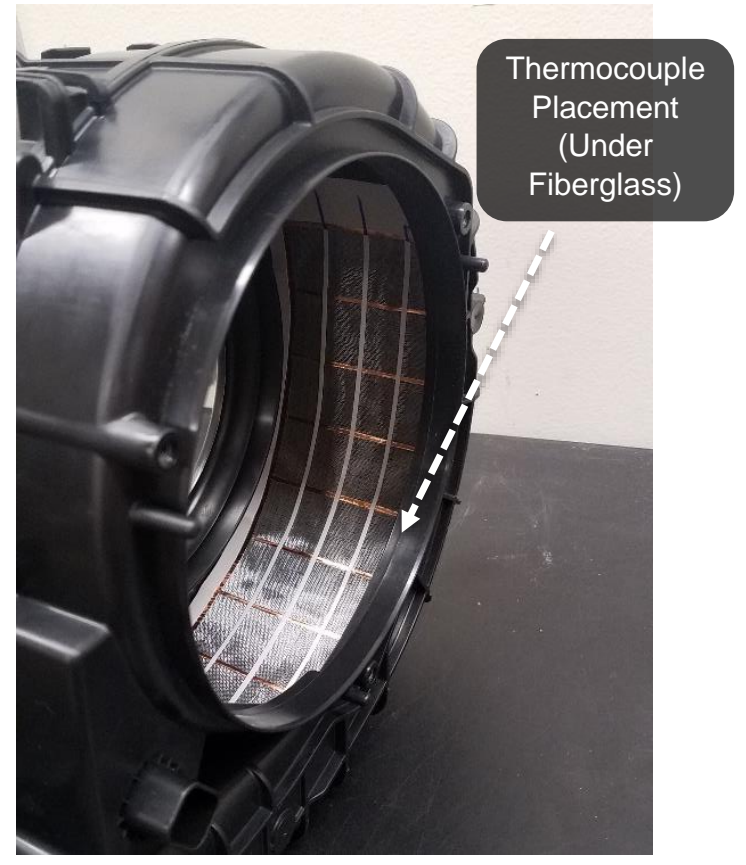

Figure 29: Thermocouple Location for FC01 Prototype Installation 


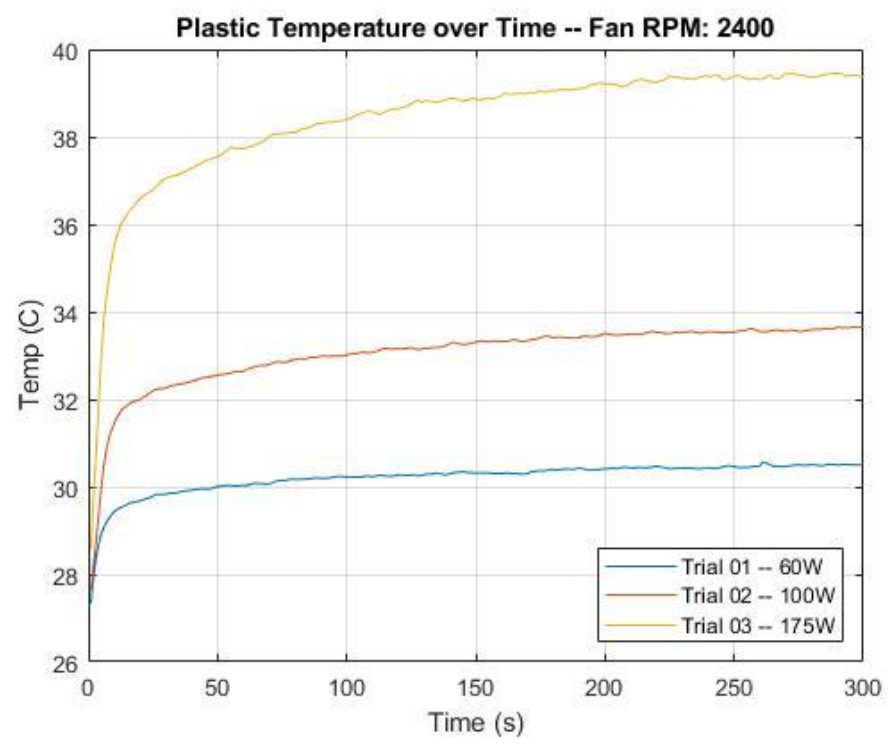

Figure 30: Temperature of Plastic in Blower Fan During FC01 Operation

The plastic temperature was well within limits even while a high amount of power was delivered to the thermophone, provided air is flowing in the fan. If the airflow is stopped however, the temperature of the plastic quickly reaches its safety threshold (Figure 31).

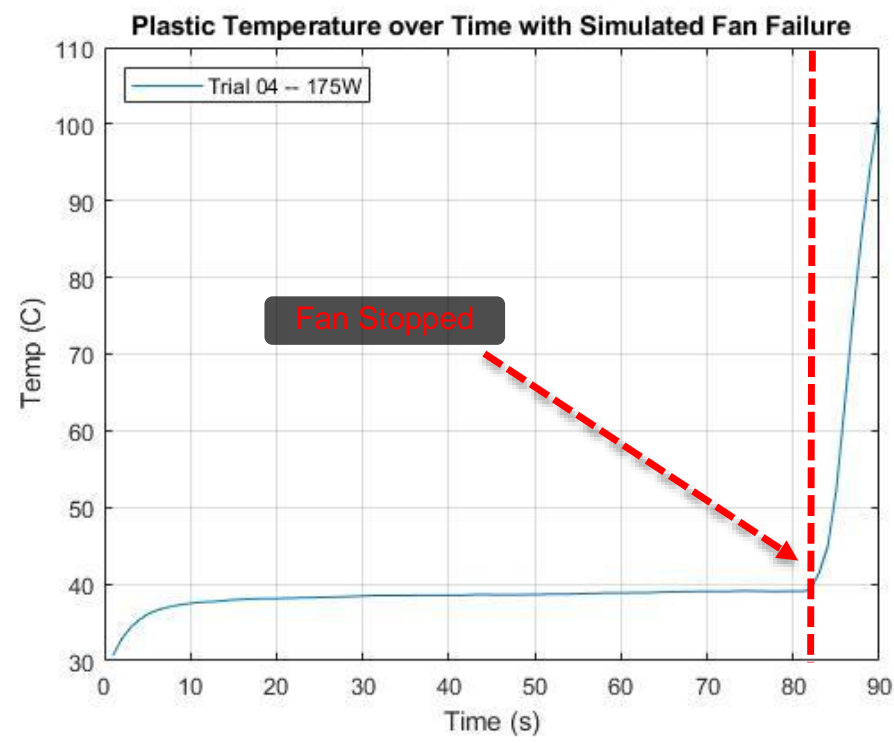

Figure 31: Temperature of Plastic in Blower Fan During FC01 During Simulated Fan Failure

\subsection{Acoustic Path Comparison Experiment}

Until this point, most experiments performed in this case study were aimed at characterizing the performance of a design or piece of equipment. This experiment was 
mean to investigate an acoustic theory, and because of this, its entry is formatted slightly differently than others in this section. Much more background is given, and an important set of calculations is laid out.

\subsubsection{Overview}

Many of the previous attempts to predict whether the CNT actuators would be acoustically powerful enough to cancel a significant amount of the fan noise ended inconclusively. After looking back through old test logs and observing strange results when comparing measurements across test setups, a new investigation into speaker characterization was launched.

When the partition-mount setup was used, the thermophones installed in the blower fan seemed to have no trouble matching the overall levels of the fan spectrum (Figure 10). For the full HVAC setup, however, the same was not true. The RC03 prototype failed to get anywhere near the overall levels of the blower fan in this configuration (Figure 17). Because of this, it was theorized that the two setups affected the fan and speaker noise in different ways, hence the name of the experiment. The full HVAC setup was thought to attenuate noise emitted by the speaker more quickly than noise caused by the fan.

In order to test this, blower fan noise and thermophone noise were measured at several locations for varying configurations of both the full HVAC and partition-mount setups. This data was then analyzed to investigate the relationship between noises recorded at different positions throughout the setups. Originally this analysis was intended to confirm the theory laid out in the last sentence of the prior paragraph.

As a disclaimer before too much time is invested in this section, the theory was discredited as violating the laws of physics. However, the analysis performed on the data did uncover a different, and important, way of thinking about the myriad of different noises related to ANC within the HVAC system.

\subsubsection{Test Configuration}

Data was collected for four system configurations. The variables which changed across the configurations were: the setup (partition vs. HVAC mount), and the noise source (RC03 prototype, FC01 prototype, or blower fan). For each measurement configuration, three microphones were placed in the free field to measure the noise source. Two of the three microphones were placed in the airflow of the blower fan or the HVAC system, while one was left outside of the airflow (Figure 32, Figure 33, Figure 34). 


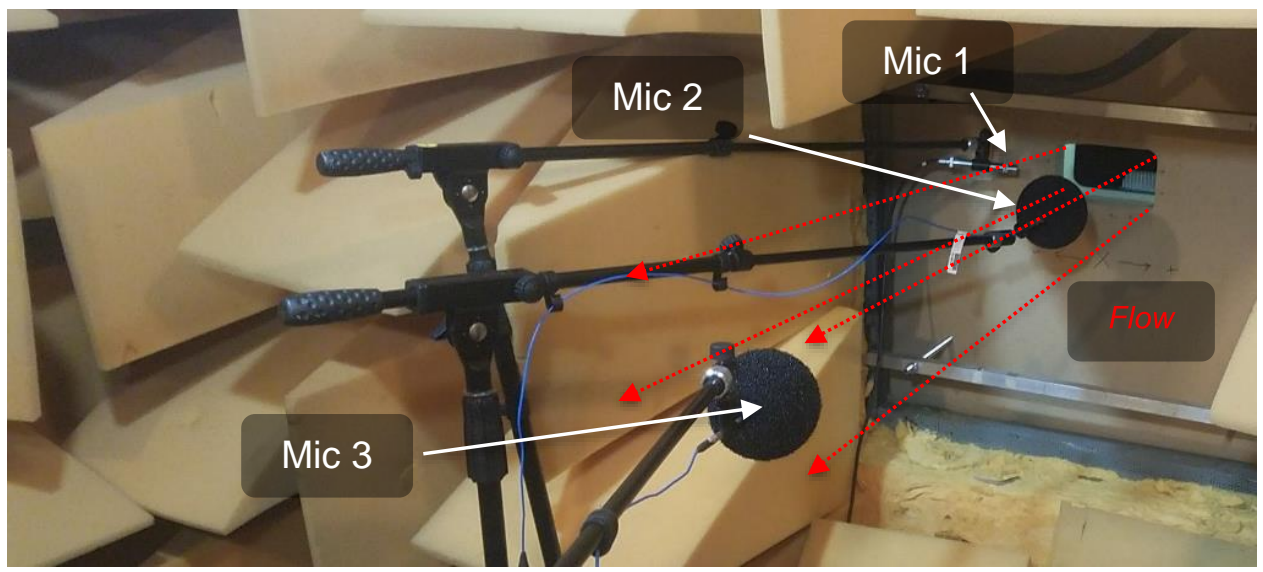

Figure 32: Measurement Microphones Configured to Characterize Sources Inside the Blower Fan in the Partition-Mount Configuration

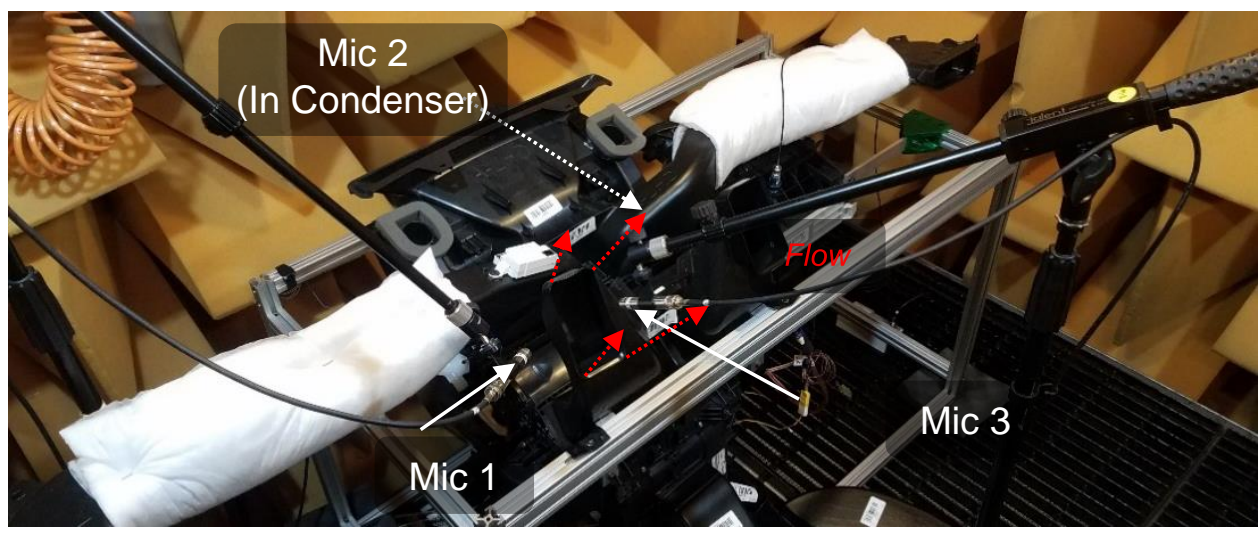

Figure 33: Measurement Microphones Configured to Characterize Sources Inside the Blower Fan in the Full HVAC Configuration

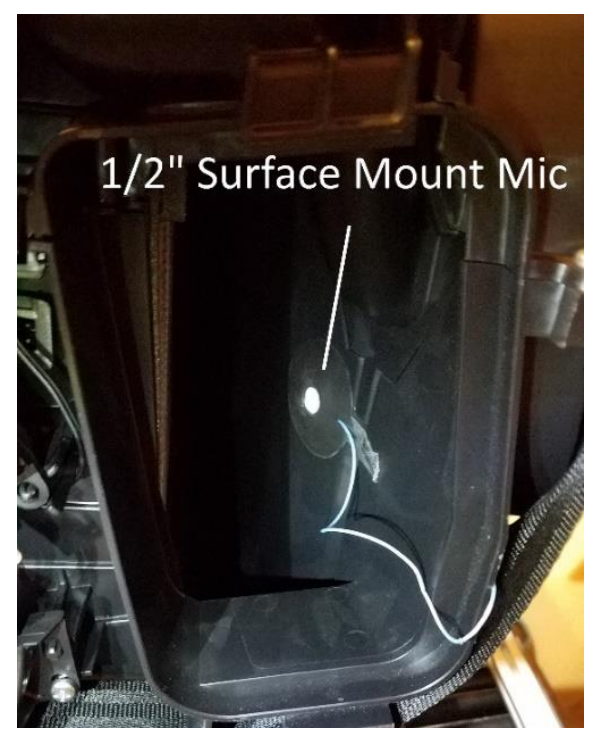

Figure 34: Detail Placement of Surface-Mount Microphone Location Used for Characterization of Sources Inside the Blower Fan in the Full HVAC Configuration 
In general across these two setups: Microphone 1 serves as a reference mic for calculations and is outside of the ducting system as well as out of the fan airflow. Microphone 3 is positioned outside the ducting system but within the airflow, and microphone 2 is positioned just past the fan outlet within the airflow. For each test setup, 20 seconds of continuous time data at $51200 \mathrm{~Hz}$ was collected from each microphone under several operating conditions, each having a different acoustic source:

Table 6: Operating Conditions for Datasets

\begin{tabular}{|c|c|c|}
\hline Trial \# & Acoustic Source -- Partition Setup & Acoustic Source -- HVAC Setup \\
\hline Trial 01 & Background Measurement & Background Measurement \\
\hline Trial 02 & RC03 Fan Noise & FC01 Fan Noise \\
\hline Trial 03 & RC03 55W White Noise & FC01 58W White Noise \\
\hline Trial 04 & RC03 100W White Noise & FC01 110W White Noise \\
\hline Trial 05 & FC01 55W White Noise & RC03 Fan Noise \\
\hline Trial 06 & FC01 105W White Noise & RC03 58W White Noise \\
\hline Trial 07 & FC01 Fan Noise & RC03 104W White Noise \\
\hline
\end{tabular}

The noise played through the speaker was band-limited white noise excited within the 200 to $800 \mathrm{~Hz}$ range. This band was chosen as it is the primary excitation range of the Aweighted blower fan spectrum from the full HVAC configuration (Figure 17).

\subsubsection{Calculations}

To show the relationship between noises measured at different locations in the system, FRF calculations were performed. The technique used for these calculations will be laid out below as a series of assertions and equations:

Linear single-sided spectra recorded by the microphones will be a combination of several different signals:

$$
\begin{gathered}
G_{1}^{\prime}=G_{1}+G_{l} \\
G_{2}^{\prime}=G_{2}+G_{m}+G_{f 2} \\
G_{3}^{\prime}=G_{3}+G_{n}+G_{f 3}
\end{gathered}
$$

Where $\mathrm{G}_{1: 3}^{\prime}$ are the signal recorded by the DAQ for respective mics, $\mathrm{G}_{1: 3}$ is the deterministic fan noise recorded for each microphone, $\mathrm{G}_{\mathrm{l}: \mathrm{m}}$ are sensor noise, \& $\mathrm{G}_{\mathrm{f} 2: 3}$ is random flow noise picked up by the microphones within the airflow. It is assumed that the deterministic fan noise will be correlated across all microphones since the test takes place in an anechoic chamber.

Performing a traditional FRF calculation ( $\mathrm{H} 1$ or $\mathrm{H} 2)$ between microphones 2 \& 3 cuts out uncorrelated noise (flow noise plus sensor noise) on only one channel. In the case where this noise is not dominant, this does not present a large issue. In the case of the blower fan noise, however, the flow noise is dominant. In this case the magnitude of a traditional FRF will have little meaning since the majority of one of the spectra used in the calculation is random. Phase results of the FRF will only depend on the relative phase of the 
deterministic components in the signals (i.e. $G_{2} \& G_{3}$ ) \& do not present a problem concerning the method of FRF calculation.

An extra microphone is used to fix this problem. An FRF consists of two components: gain and phase. The desired result for this experiment is the ratio of $G_{2} \& G_{3}$. By taking the three possible crosspowers ${ }^{2}$ in the system this ratio can be determined.

$$
\begin{gathered}
G_{12}^{\prime}=\left[G_{1}+G_{t}\right] \times\left[G_{2}+G_{m}+G_{f 2} f\right]^{*} \\
G_{13}^{\prime}=\left[G_{1}+G_{t}\right] \times\left[G_{3}+G_{n}+G_{f 3}\right]^{*} \\
G_{23}^{\prime}=\left[G_{2}+G_{m}+G_{f 2}\right] \times\left[G_{3}+G_{n}+G_{f 3}\right]^{*}
\end{gathered}
$$

Taking advantage of the complex nature of the recorded spectra, the influence of the crossed-out terms in the equation above will trend towards a value of zero with averaging. The equations above simplify to:

$$
\begin{aligned}
G_{12}^{\prime} & =G_{1} \times G_{2}{ }^{*} \\
G_{13}^{\prime} & =G_{1} \times G_{3}{ }^{*} \\
G_{23}^{\prime} & =G_{2} \times G_{3}{ }^{*}
\end{aligned}
$$

And the magnitudes of the crosspowers are as follows:

$$
\begin{aligned}
& \left|G_{12}^{\prime}\right|=\left|G_{1}\right| \times\left|G_{2}\right| \\
& \left|G_{13}^{\prime}\right|=\left|G_{1}\right| \times\left|G_{3}\right| \\
& \left|G_{23}^{\prime}\right|=\left|G_{2}\right| \times\left|G_{3}\right|
\end{aligned}
$$

Since $\left|G_{12}^{\prime}\right|,\left|G_{13}^{\prime}\right|, \&\left|G_{23}^{\prime}\right|$ are all known quantities, the three equations above represent a simple 3-equation 3-unknown system. This allows for the calculation of the exact magnitudes of the deterministic noise $G_{1: 3}$ from Eqn. Set 02 . It should be noted that similar calculations could be used to find the absolute phase of all three deterministic signals; this is not done here since only the relative phase between $G_{2} \& G_{3}$ is of importance.

After eliminating the random sensor and flow noise components with averaging, the ratio of $\left|G_{2}\right| \&\left|G_{3}\right|$ computed from Eqn. Set 05 can be used as the gain of the FRF. The phase of the FRF is simply the angle of complex-valued $G_{23}^{\prime}$.

\subsubsection{Results}

Before the results are laid out some context for ongoing use of the term "FRF" within this experiment should be given. It is used in a purely mathematical sense. Typically, an FRF

${ }^{2}$ Crosspower and autopower spectra computed using a Hanning window and the energy correction factor 
represents the relationship between the input of a system and its output. In the case here, nowhere near enough measurements are being made such that microphone signals could represent inputs or outputs to an acoustic model of the HVAC system. FRF calculations are performed for this experiment for comparative, rather than quantitative analysis.

FRF calculations (new formulation) were done for all three noise sources, for each setup configuration (Figure 35 \& Figure 36). Results for blower fan noise were omitted from Figure 36 due to extremely high levels of noise, which were obscuring the plot.
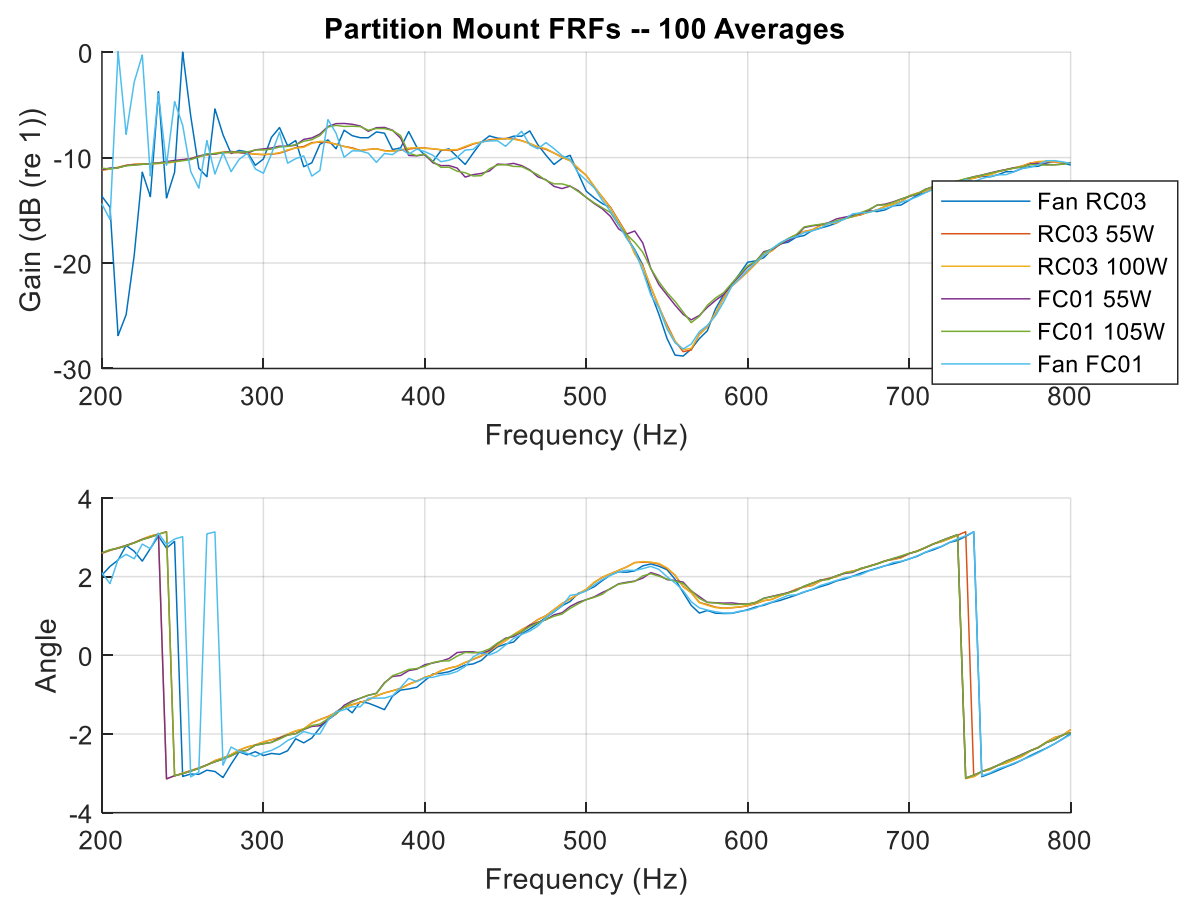

Figure 35: Gain and Phase Between Responses of Mics. 2 \& 3 for Three Noise Sources in the PartitionMount Setup 

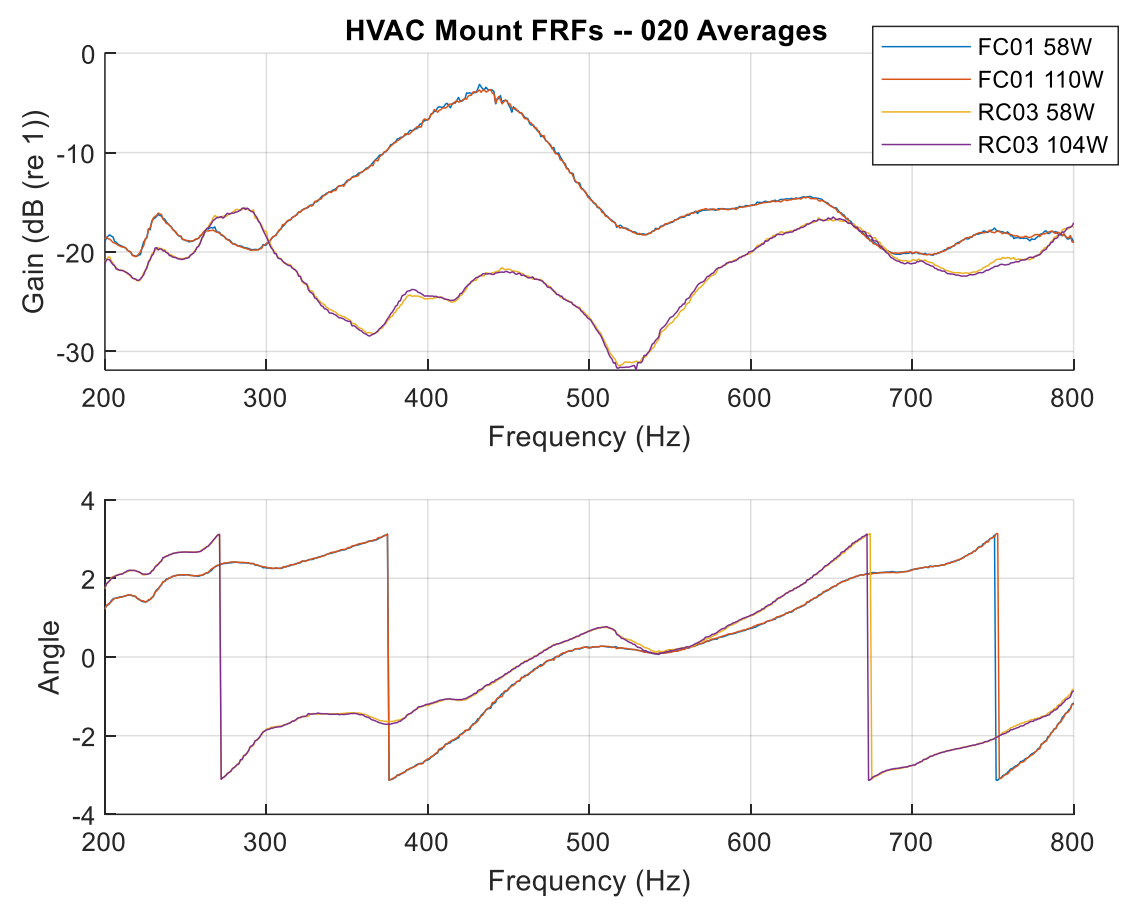

Figure 36: Gain and Phase Between Responses of Mics. 2 \& 3 for Two Noise Sources in the Partition-Mount Setup

All datasets collected were also analyzed using traditional FRF calculations $(\mathrm{H} 1$ formulation). Having results from both formulations is advantageous since they differ in their sensitivity to randomness within the microphone spectrums. Agreement across calculation methods indicates that the spectra are dominated by deterministic components. Discrepancy across calculation methods indicates that the spectra are dominated by random components. Comparison of results from the two FRF calculation methods for all sources across the HVAC setup trials, and for the fan in the partition-mount setup trials, can be seen below (Figure 37 \& Figure 38 ). 

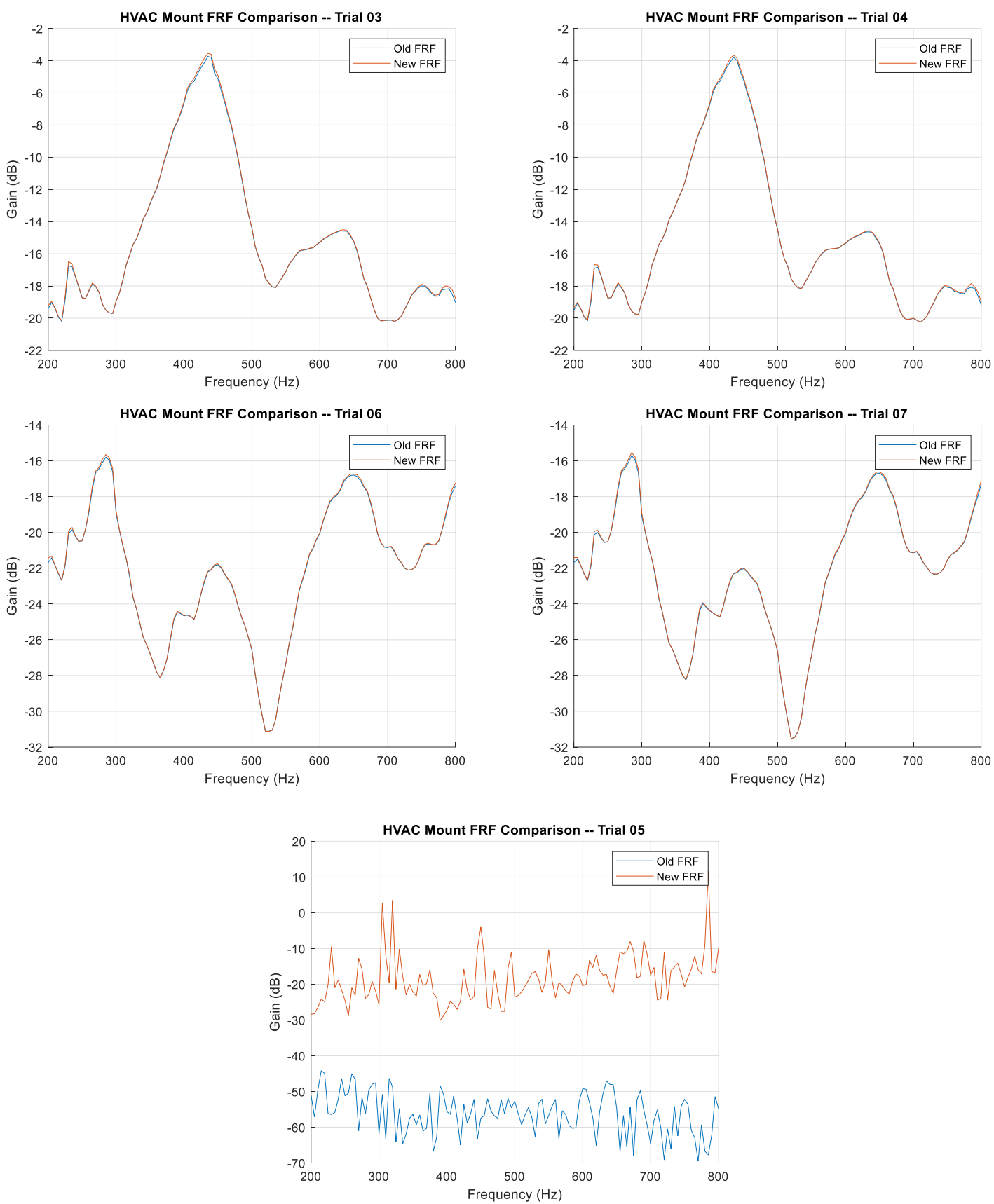

Figure 37: Comparison of FRF Calculation Methods for Different Noise Sources in the HVAC Setup 


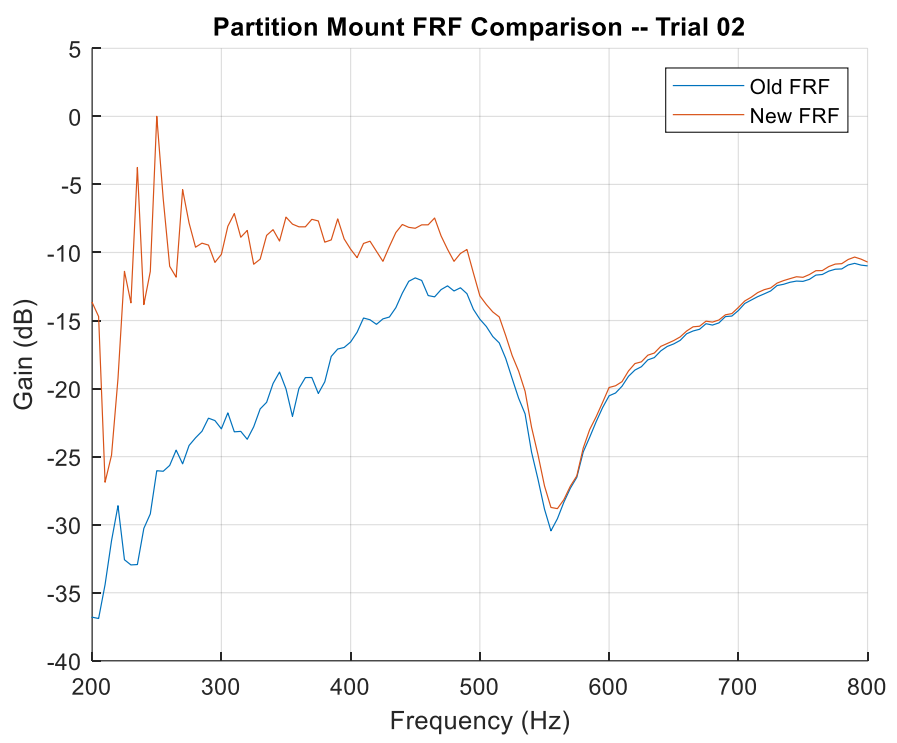

Figure 38: Comparison of FRF Calculation Methods for Blower Fan Noise in the Partition-Mount Setup

Eqn. Set 05 was used to find the amplitude of the deterministic portion of various autopower spectra measured throughout testing. These magnitude spectra were then overlaid with traditional autopower spectra. Comparisons of such results for different noise sources (blower fan vs. arbitrary thermophone) were made for each setup (Figure 39 \& Figure 40)
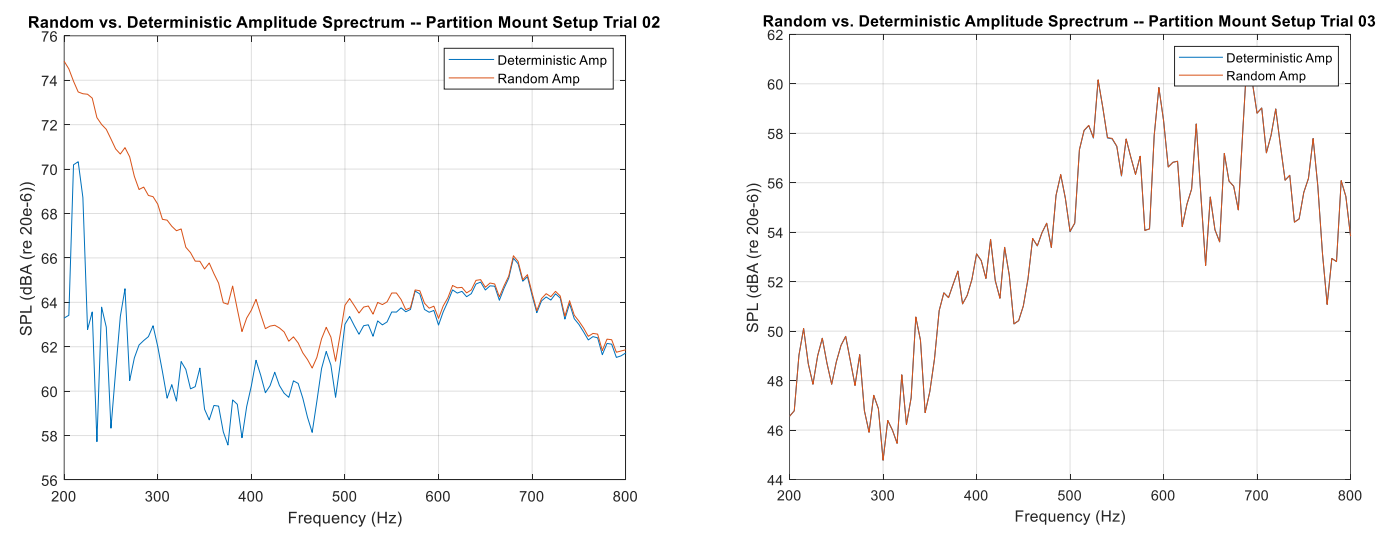

Figure 39: Comparison of Deterministic Magnitude Spectra to Autopower Spectra for Two Noise Sources in the Partition-Mount Setup 

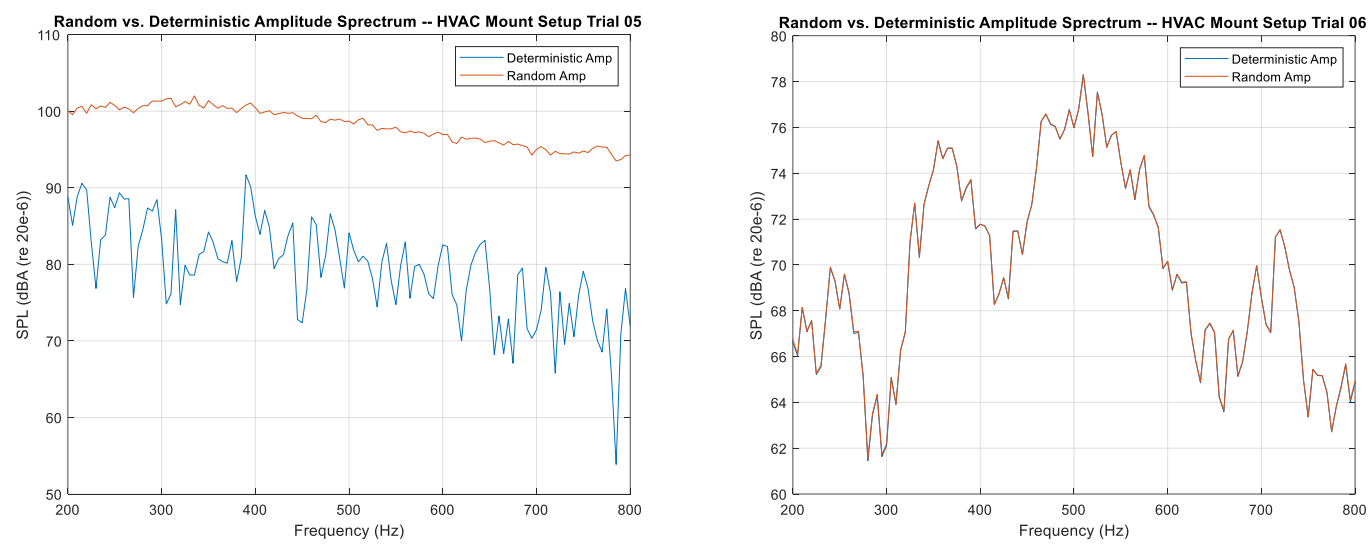

Figure 40: Comparison of Deterministic Magnitude Spectra to Autopower Spectra for Two Noise Sources in the Full HVAC Setup

\subsection{Initial Cancellation Testing with the Tiger WIFI-S Controller}

The test trials to characterize the performance of the Tiger WIFI-S had a very broad scope. For increased clarity, the ANC testing done with this controller will be split up into four chronological rounds.

\subsubsection{First Testing Phase}

The first round of testing was aimed at getting preliminary experience using the Tiger Controller. For most of these tests a unique test setup was used. One disturbance loudspeaker and two actuators (only one was used at a time) were placed in the anechoic chamber, along with two $1 / 2$ " measurement microphones. The actuator varied between a loudspeaker and a CNT thermophone (Figure 41). 


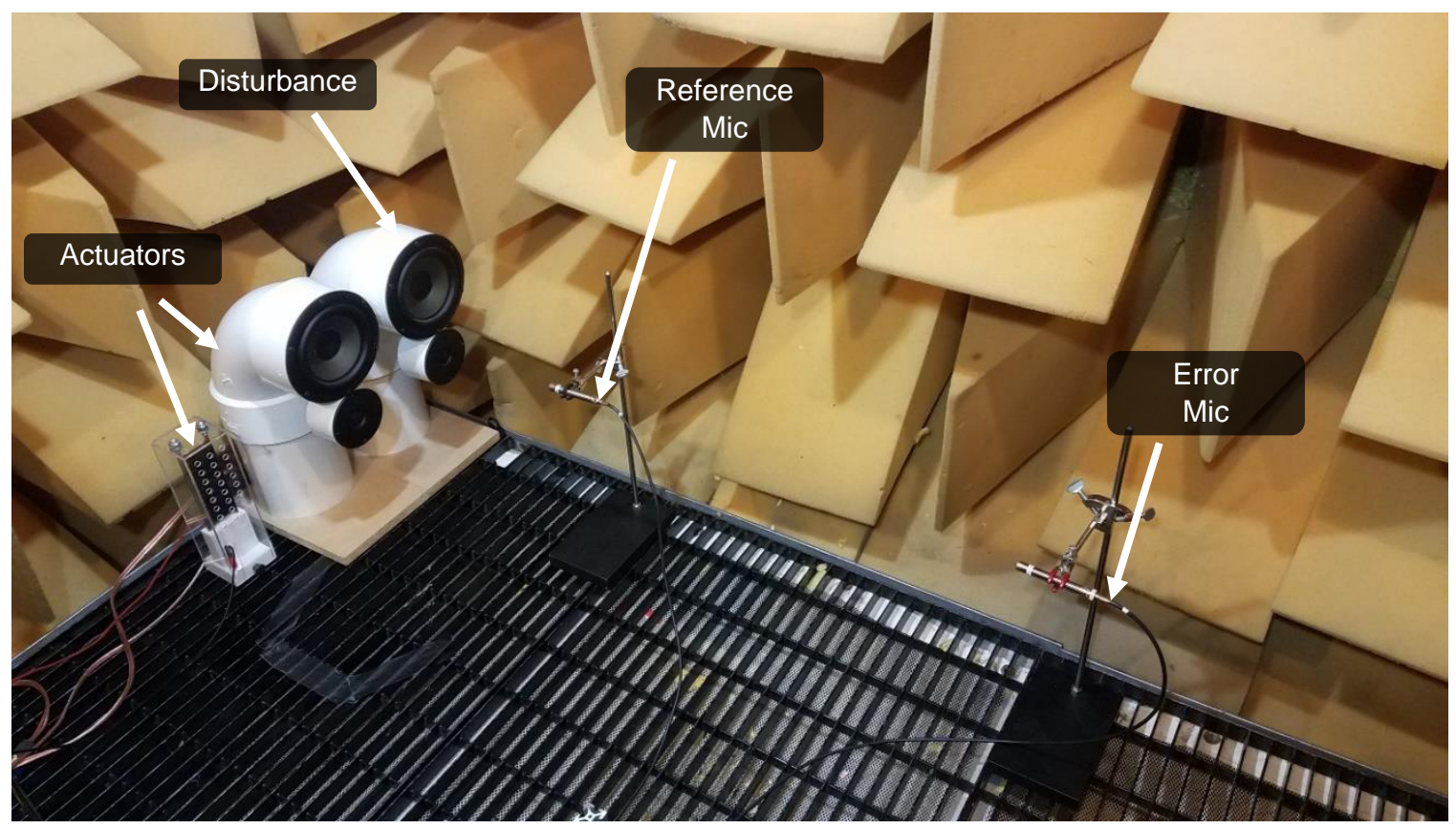

Figure 41: Microphone, Disturbance, and Actuator Placement for the First Free-Field ANC Tests Performed with the Tiger WIFI-S Controller

Disturbance noises across the trials varied, including pure tones at different frequencies and broadband white noise signals. Many issues with the equipment used for the CNT thermophone were discovered during this testing phase. The tests run here were more important for getting the actual ANC configuration working than for gauging performance, so data will not be reported. At the conclusion of this testing phase, both tonal and broadband noises were cancellable using a loudspeaker as an actuator; CNT was not yet working as the actuator.

\subsubsection{Second Testing Phase}

After getting comfortable with the simple test setup explored in phase 01, a more realistic test setup that incorporated fan noise was used. The anechoic chamber was reconfigured into the partition-mount setup. Placement of the $1 / 2$ " error and reference microphones, as well as the actuator (3" loudspeaker) was recorded (Figure 42). Care was taken to ensure that the measurement microphones did not experience airflow in any configuration. 


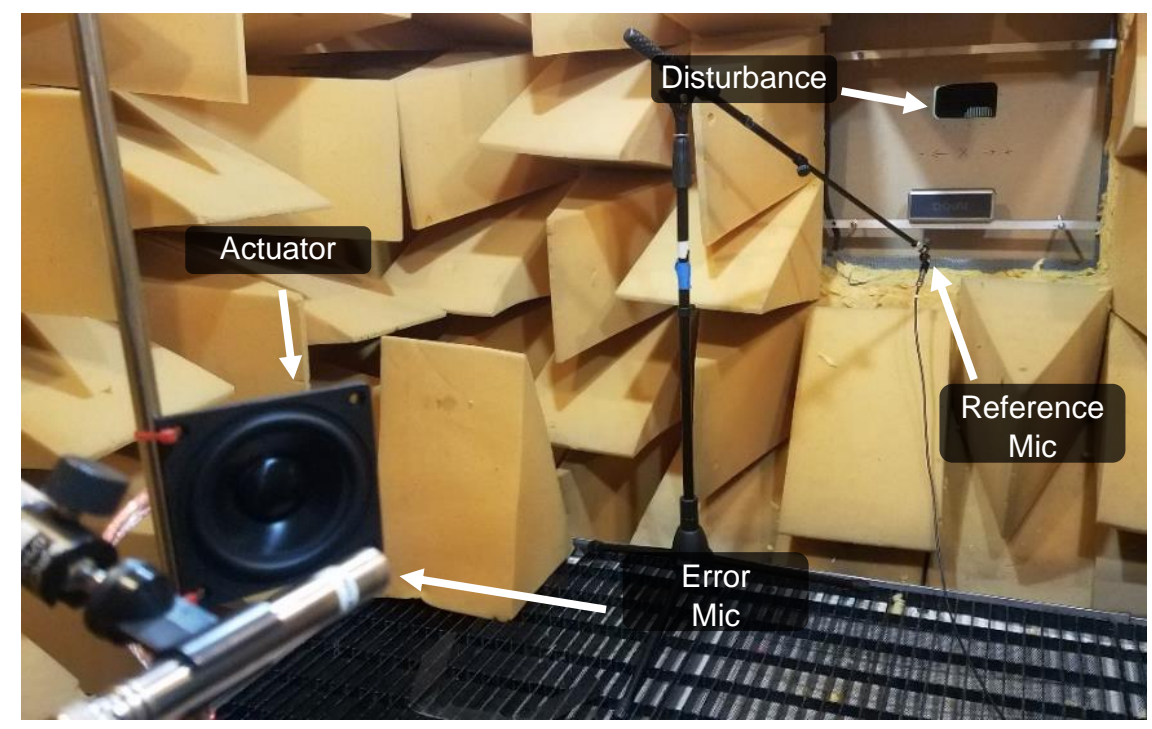

Figure 42: Microphone, Disturbance, and Actuator Placement for the First Free-Field ANC Tests Performed On Blower Fan Noise with the Tiger WIFI-S Controller

The system was tuned for cancellation using a Bluetooth speaker as a disturbance, but cancellation trials were also performed using fan noise as the disturbance. The Tiger WIFI$S$ was found to be able to reduce the overall SPL of blower fan noise at the error microphone location by $6.8 \mathrm{dBA}$ (Figure 43).

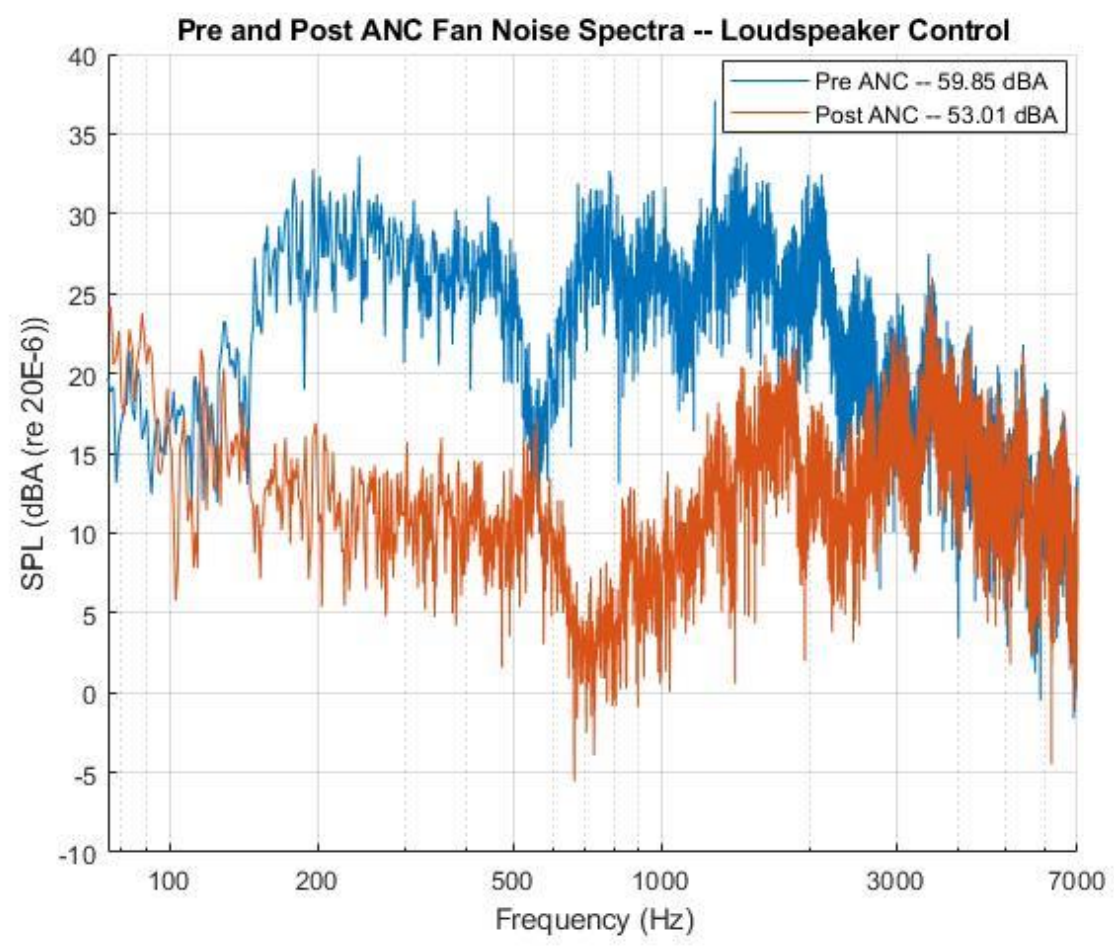

Figure 43: Cancellation of Blower Fan Noise with Loudspeaker Actuator - Tiger WIFI-S Cancellation Testing Phase 02 


\subsubsection{Third Testing Phase}

At this point in time a new amplitude modulator board had been developed, so ANC trials using CNT actuators became possible. Testing to explore this was performed using a setup very similar to that of the previous experiment (Figure 42). The actuator and error microphone ( $1 / 2$ " free field) were positioned in an anechoic chamber, with the blower fan in the partition-mount setup. The reference microphone $(1 / 2$ " free field) was placed directly underneath the opening of the partition to be as close to the opening as possible without experiencing airflow from the fan (Figure 44 \& Figure 45).

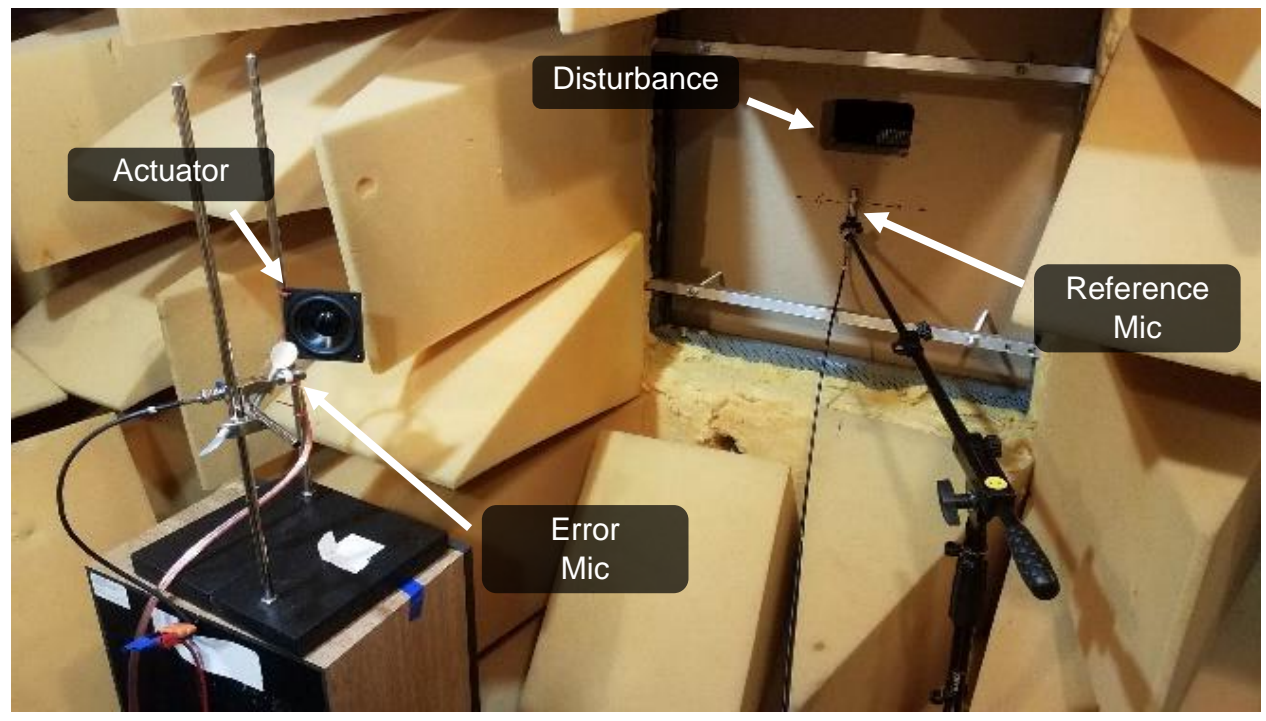

Figure 44: Microphone, Disturbance, and Loudspeaker Actuator Placement for Free-Field ANC Tests Performed On Blower Fan Noise with the Tiger WIFI-S Controller

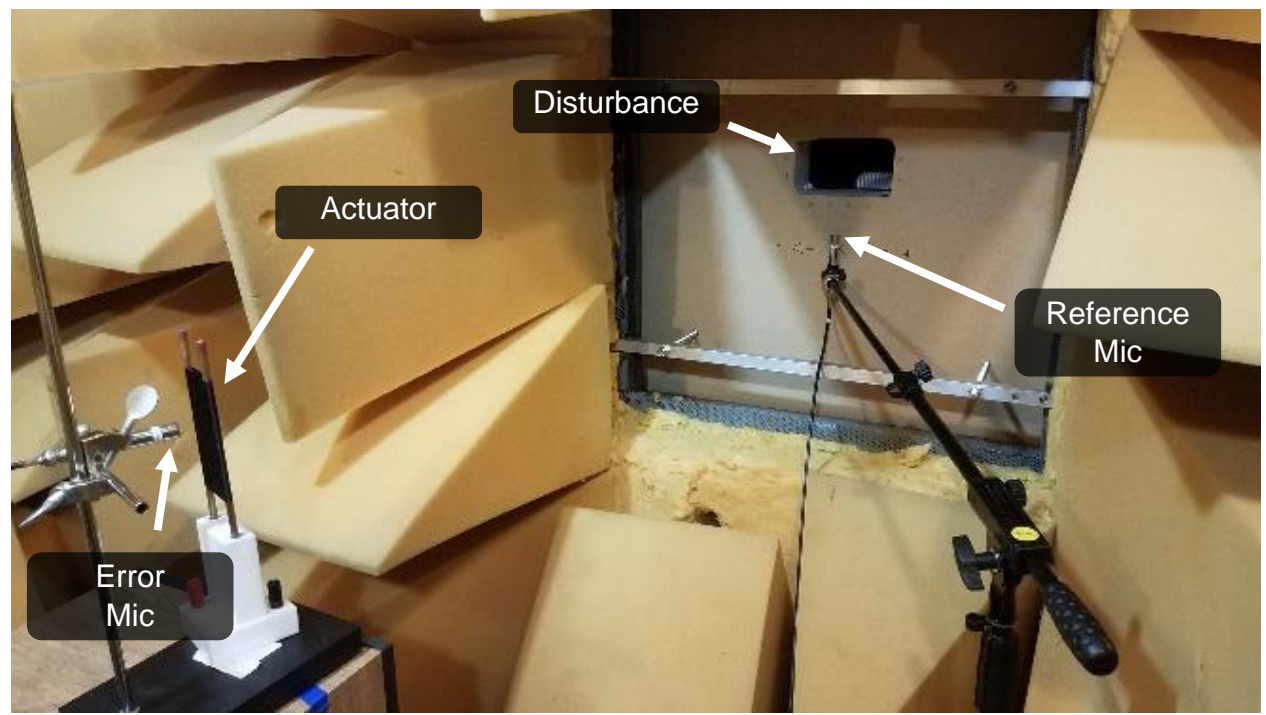

Figure 45: Microphone, Disturbance, and Thermophone Actuator Placement for Free-Field ANC Tests Performed On Blower Fan Noise with the Tiger WIFI-S Controller 
In these two configurations several things were tested. First, ANC performance was compared across the two different actuators. Second, the actuators and error microphone ${ }^{3}$ were moved incrementally closer to the reference microphone in order to find the minimum spacing required between the two microphones in the system. Finally, the error microphone was moved incrementally into the airflow in order to investigate the relationship between microphone coherence and cancellation results.

The results of three ANC trials for each actuator were recorded and compared (Table 7, Figure 46, \& Figure 47).

Table 7: Comparison of Blower Fan Noise Cancellation in the Free Field using CNT and Loudspeaker actuators

\begin{tabular}{|c|c|c|}
\hline Trial Number & $\begin{array}{c}\text { Loudspeaker Control } \\
\text { Cancellation (dBA) }\end{array}$ & $\begin{array}{c}\text { CNT Control } \\
\text { Cancellation (dBA) }\end{array}$ \\
\hline 01 & 4.6 & 3.9 \\
\hline 02 & 4.8 & 3.7 \\
\hline 03 & 4.9 & 3.4 \\
\hline
\end{tabular}

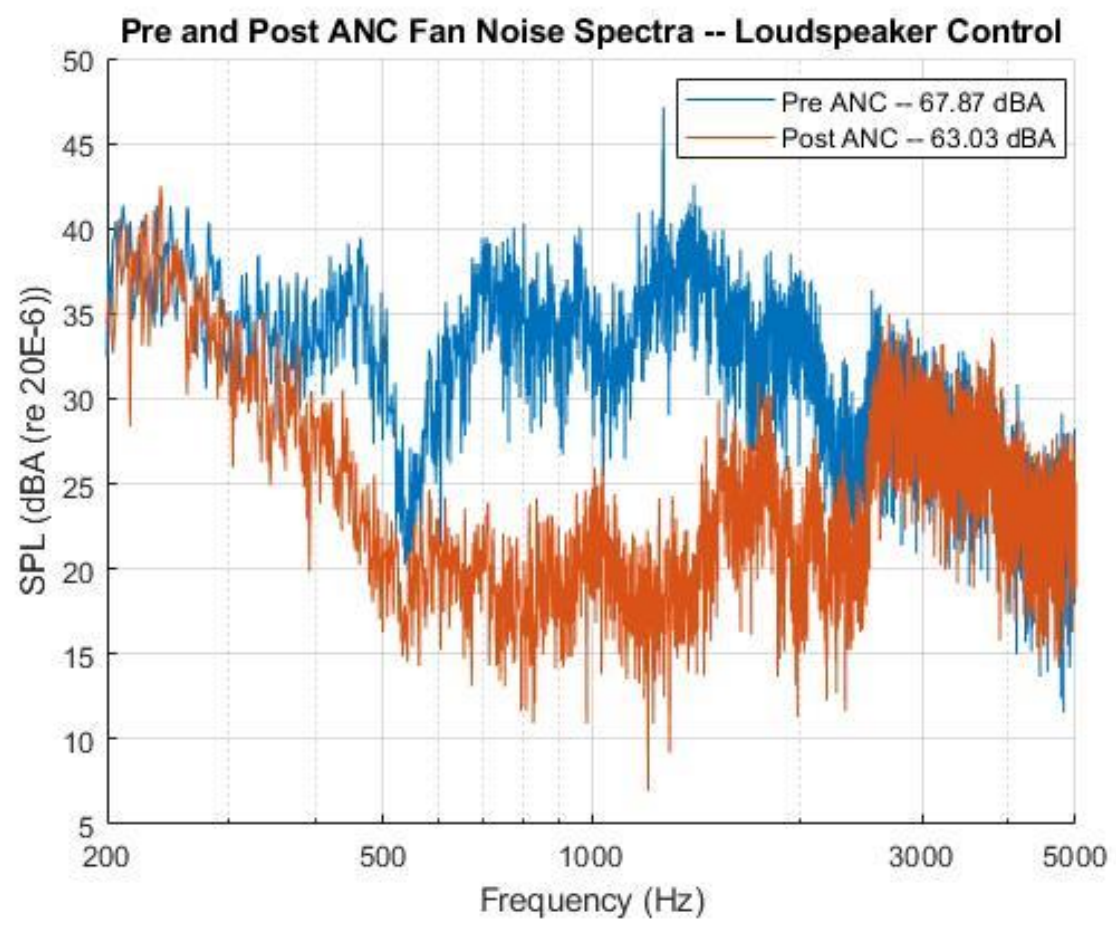

Figure 46: Pre and Post ANC Spectra Showing Cancellation of Fan Noise in the Free Field using a Loudspeaker Actuator and the Tiger WIFI-S Controller

${ }^{3}$ The positions of the error microphone and actuator were kept constant relative to one another 


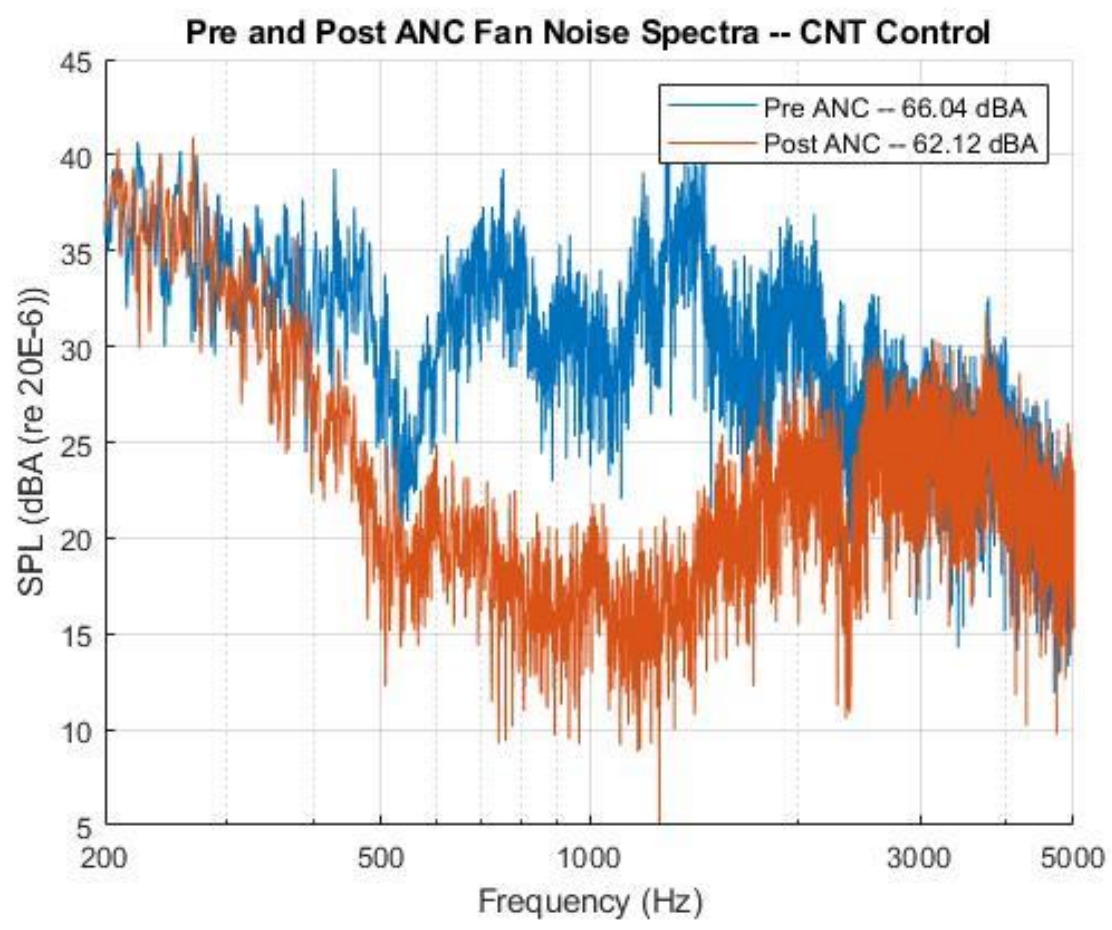

Figure 47: Sample Spectrum from Phase 03 ANC Testing - CNT Actuator

It was found that the minim microphone spacing of $90 \mathrm{~cm}$ which could be achieved using this test setup (Figure 44 \& Figure 45) was not enough to impact cancellation performance in a meaningful way. More tests using a different setup that allowed for closer microphone spacing was done later to determine effects of causality on ANC performance.

\subsubsection{Fourth Testing Phase}

Once broadband cancellation was achieved in the free field using the partition-mount setup, it seemed logical to check whether the Tiger WIFI-S could also be used on the full HVAC configuration. The final phase of the Tiger WIFI-S initial cancellation testing was an attempt to do this. Ultimately, all trials in this phase proved unsuccessful, and no cancellation of the fan noise or broadband white noise was achieved. It is still worth mentioning some of the troubleshooting done on this setup, however, to show what did not work.

Two $1 / 2$ " measurement microphones and the loudspeaker actuator were all placed in the free field for these tests (Figure 48). Two disturbances were used for these cancellation trials, the fan noise itself, and broadband noise played through a loudspeaker mounted onto the RC03 prototype CNT chamber (Figure 49). 


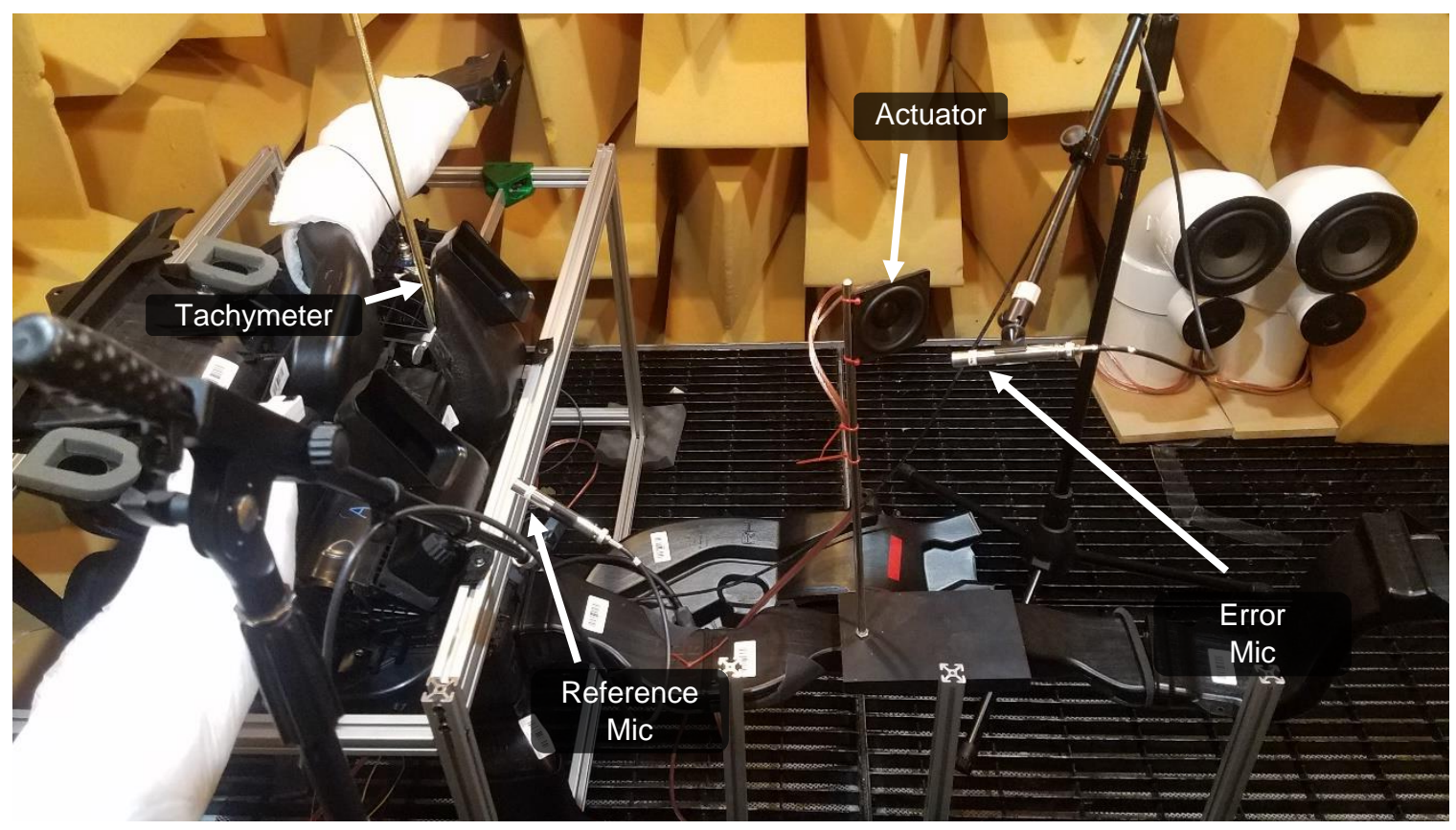

Figure 48: Tachymeter, Microphone, Disturbance, \& Actuator Configuration for Cancellation of HVAC Noise at a Free-Field Location

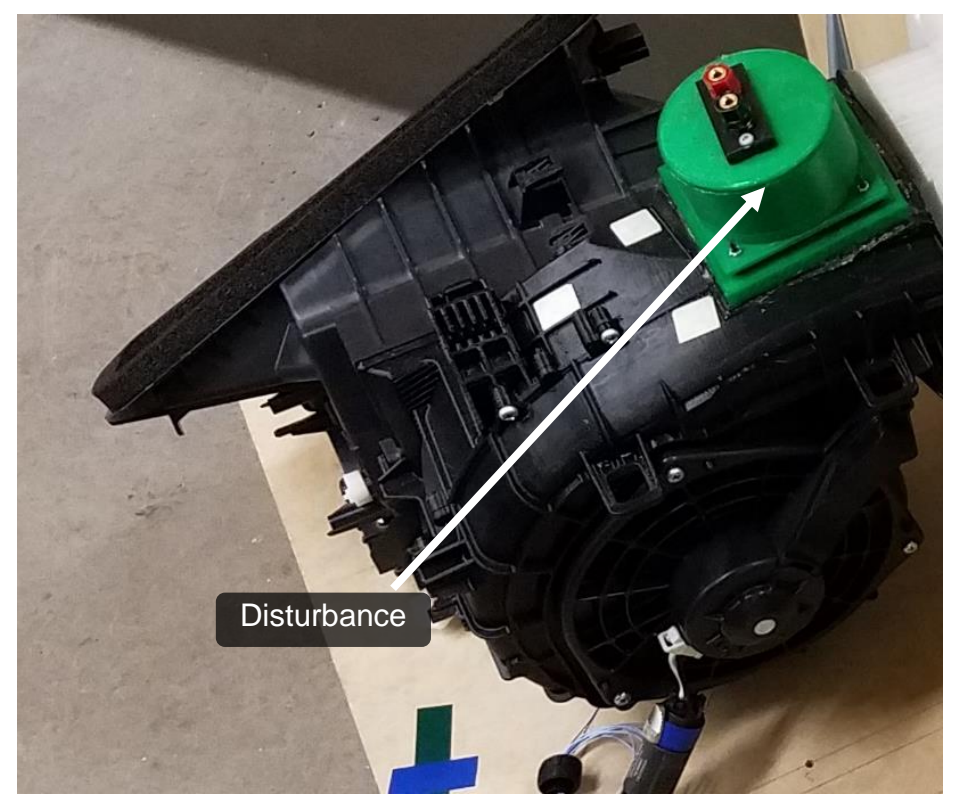

Figure 49: Detail of Loudspeaker Disturbance Placement on Blower Fan

The first cancellation trials were done using the blower fan noise as the disturbance source. Several transducers were used to supply the reference signal, a $1 / 2$ " mic in the free field (Figure 48), a laser tachymeter measuring fan speed, and a surface-mount microphone mounted in the evaporator near the blower fan exhaust (Figure 34). No transducer produced good results. 
It was noted at this point that the coherence between all these transducers and the error microphone was poor. Best of the three reference transducers in this sense was the free field mic, so this transducer was used exclusively going forward. Troubleshooting efforts were then focused on improving the coherence between the two microphones. Reducing the spacing between the microphones had a positive effect on coherence (Figure 50), but ultimately did not yield any better cancellation results.

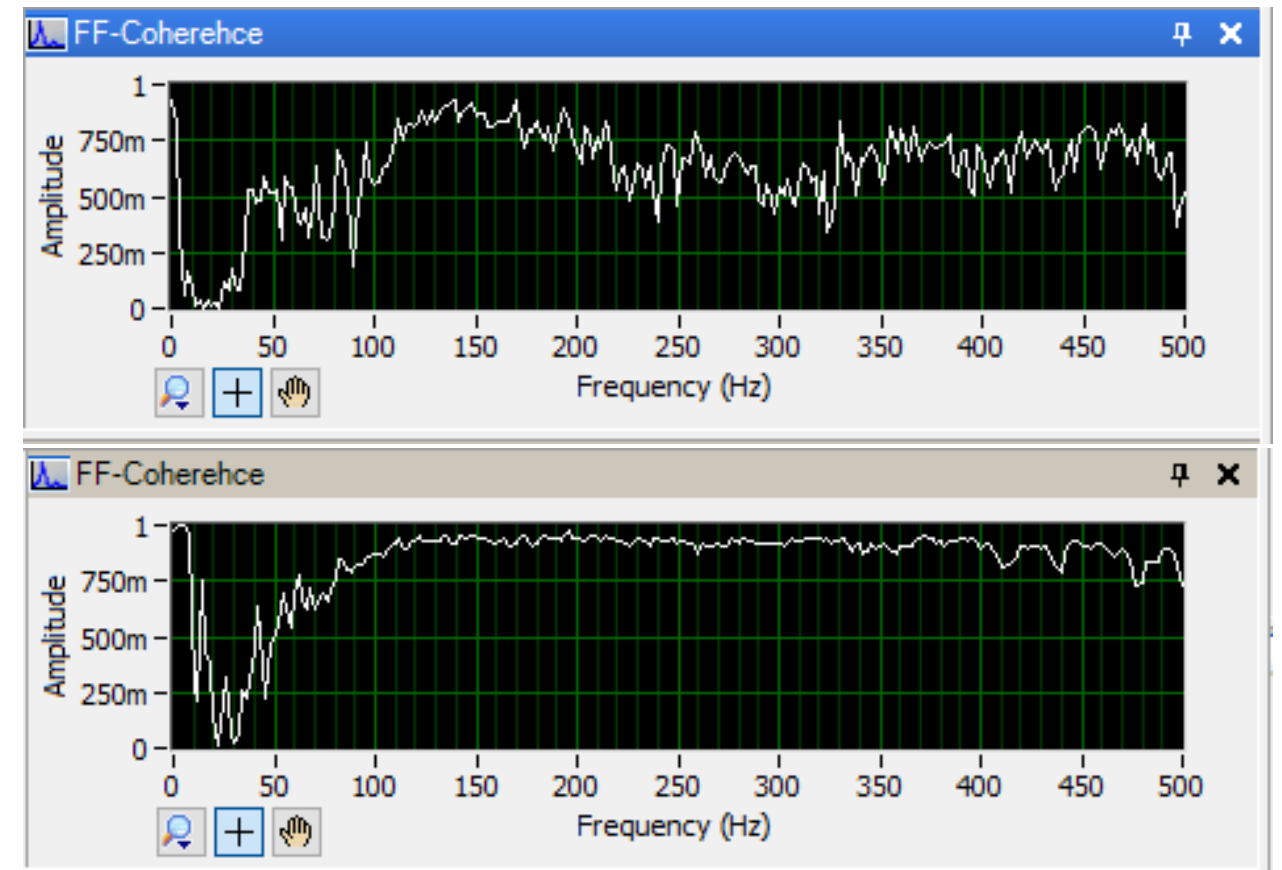

Figure 50: Comparison of Microphone Coherence Before and After Tuning Microphone Spacing

Finally, cancellation was tried using the same configuration, except with a loudspeaker serving as the disturbance. This too was found to be ineffective. More comments and discussion on the ineffectiveness of these test trials can be found later in the report.

\subsection{In-Duct Cancellation Testing with the Tiger WIFI-S}

After getting comfortable with the Tiger WIFI-S in the previous experiment, it was shown that the controller could be effective at attenuating broadband noise. However, it was found to be ineffective when applied to the full HVAC setup. In the interest of reducing the complexity of the ANC system from an acoustics and airflow perspective, experimentation was moved to a simpler test setup for this test suite. Broadband noise propagating through one length of ducting, 4" PVC pipe in this case, was the target for cancellation. The disturbance was provided by either a disturbance loudspeaker or the blower fan for different trials (Figure 51 \& Figure 52). The test setup developed for this suite was also used for an ANC technology demo at the project sponsor's facility. 


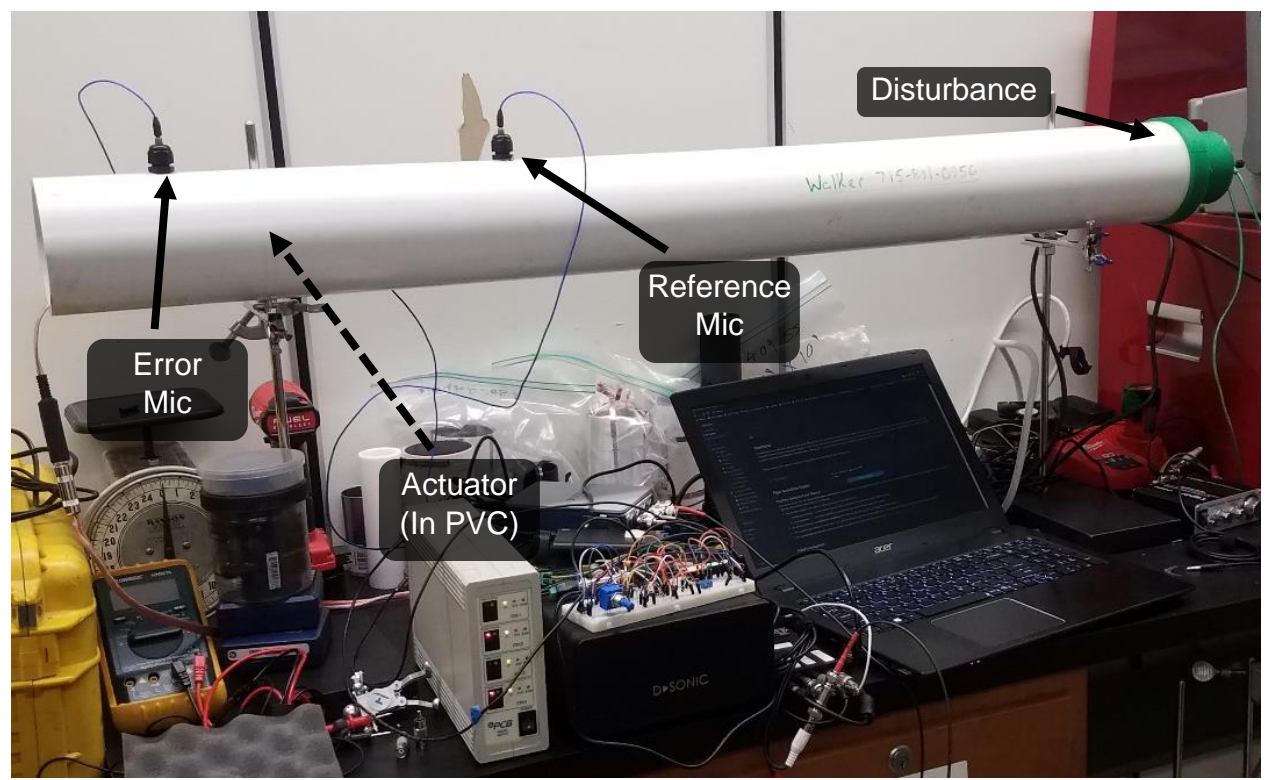

Figure 51: Configuration of Demo Ducted Setup for Cancellation Testing with a Loudspeaker Disturbance \& CNT Actuator

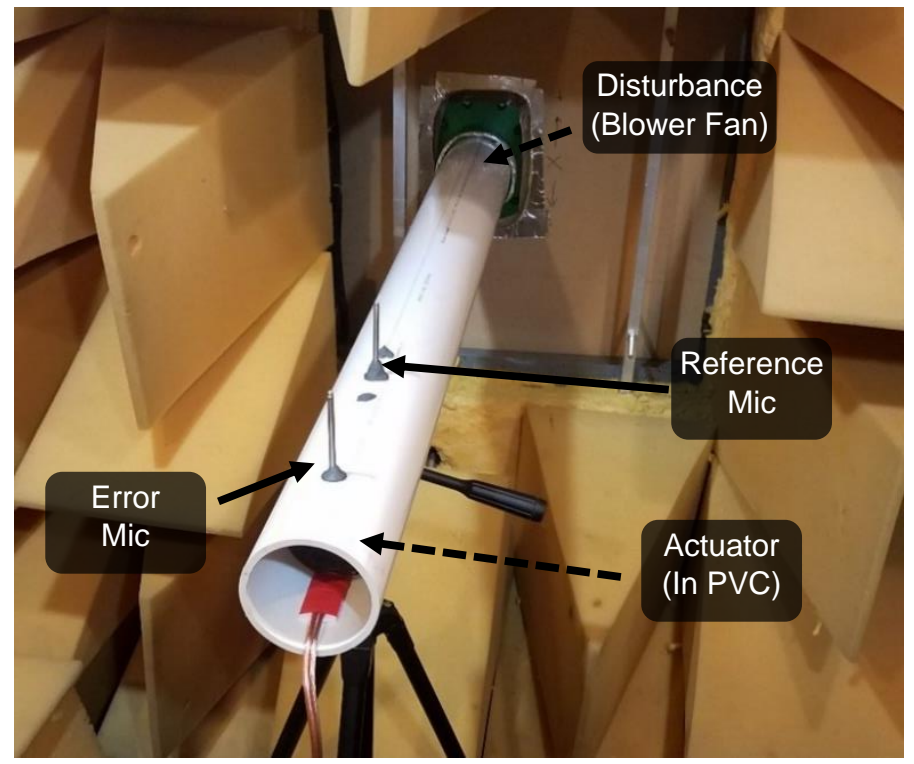

Figure 52: Configuration of Ducting Setup for Cancellation Testing with a Blower Fan Disturbance

Two 1/4" microphones were flush-mounted to the inside of the PVC pipe to serve as reference and error transducers. The annular Teflon thermophone was used as the actuator.

Controller parameters and disturbance signal content were varied until a subjective "best case" for cancellation was found. Broadband pink noise played through the loudspeaker was used as the disturbance, the Tiger WIFI-S was set to sample at $16000 \mathrm{~Hz}$, and 4096 model and filter taps were used for ANC. It is important to note that for this best-case 
scenario the reference signal was acquired by measuring directly from the disturbance signal, not by using the microphone (Figure 53).

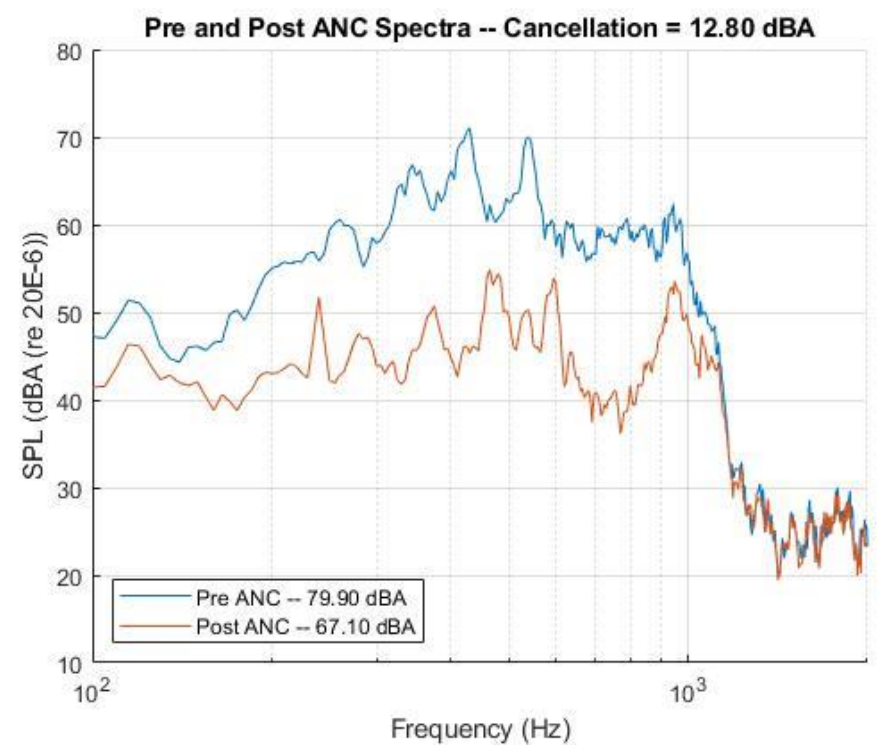

Figure 53: Best-Case Scenario for Broadband Cancellation in a Ducted Environment-Reference Signal Acquired from a Direct Electrical Line to the Disturbance Loudspeaker

Tests performed using a microphone as the reference signal were not as effective as when using a direct electrical connection to the disturbance loudspeaker to provide the reference. It was observed that reduction in the spectrum was limited to areas where pipe modes were present (Figure 54).

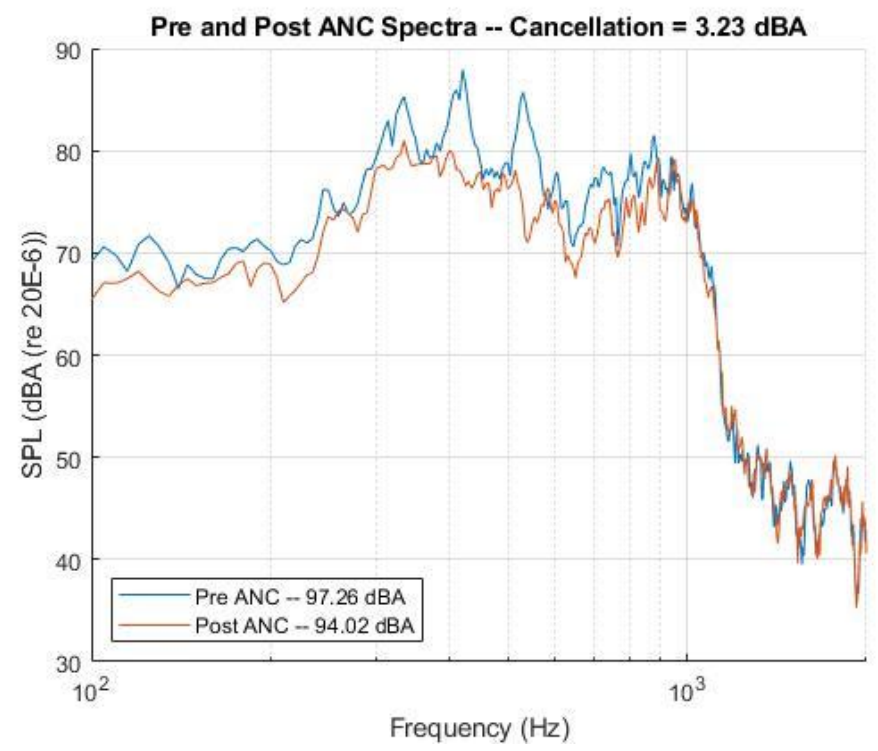

Figure 54: In-Duct Broadband Cancellation with Marginal Effectiveness -Reference Signal Acquired from a Flush-Mounted 1/4" Microphone Within the Ducting 
Cancellation trials using the blower fan noise as a disturbance were also attempted, but no reduction in noise levels was achieved in this setup configuration (Figure 52). Data recorded for these trials was used instead to compute the coherence between the two microphones. The blower fan was set to varying speeds, and at each speed different treatments to the microphone flush-mount recessions were applied. Layers of mylar film were applied to these recessions to gauge their effects on microphone coherence (Figure $55)$.
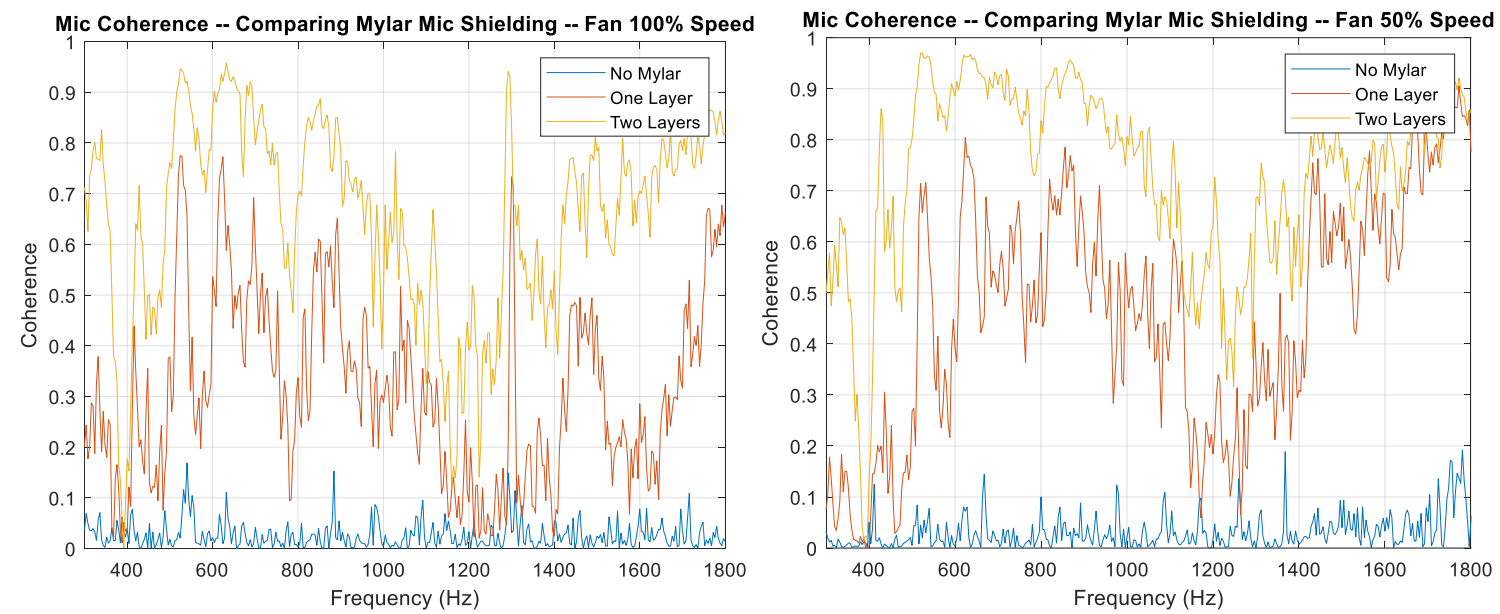

Figure 55: Comparison of Microphone Coherence with Differing Mylar Film Applications and Blower Fan Speeds

\subsection{Testing the Effects of Causality, Coherence, and Feedback on ANC Performance}

This test suite was motivated by observing the limited effectiveness of the Tiger WIFI-S in controlling broadband noise in a high-airflow ducted environment. Until this point all trials within the 4" PVC duct setup using the blower fan as the disturbance (Figure 52) were unsuccessful in reducing the noise level at the duct exit. It was theorized that two characteristics of the ANC/ducting system were behind this; microphone coherence and system causality. To test this, the relationships between cancellation performance and these two system characteristics were explored.

A new test stand was constructed so that the setup and configuration changes across these experiments would be faster. Two 1/2" microphones and the annular Teflon thermophone were attached to an aluminum beam using clips that are free to slide along the length of the beam. The beam is pointed at a disturbance source; using the Tiger WIFI$S$ controller, noise travelling past the first (reference) microphone is cancelled by the thermophone at the second (error) microphone. For the first set of experiments, the beam was mounted on top of a camera tripod; this ANC configuration will be referred to as the "free-field cancellation stand" throughout. For the first set of tests in this experimental 
suite, the free-field cancellation stand was aimed at loudspeakers which provided the disturbance noise. (Figure 56).

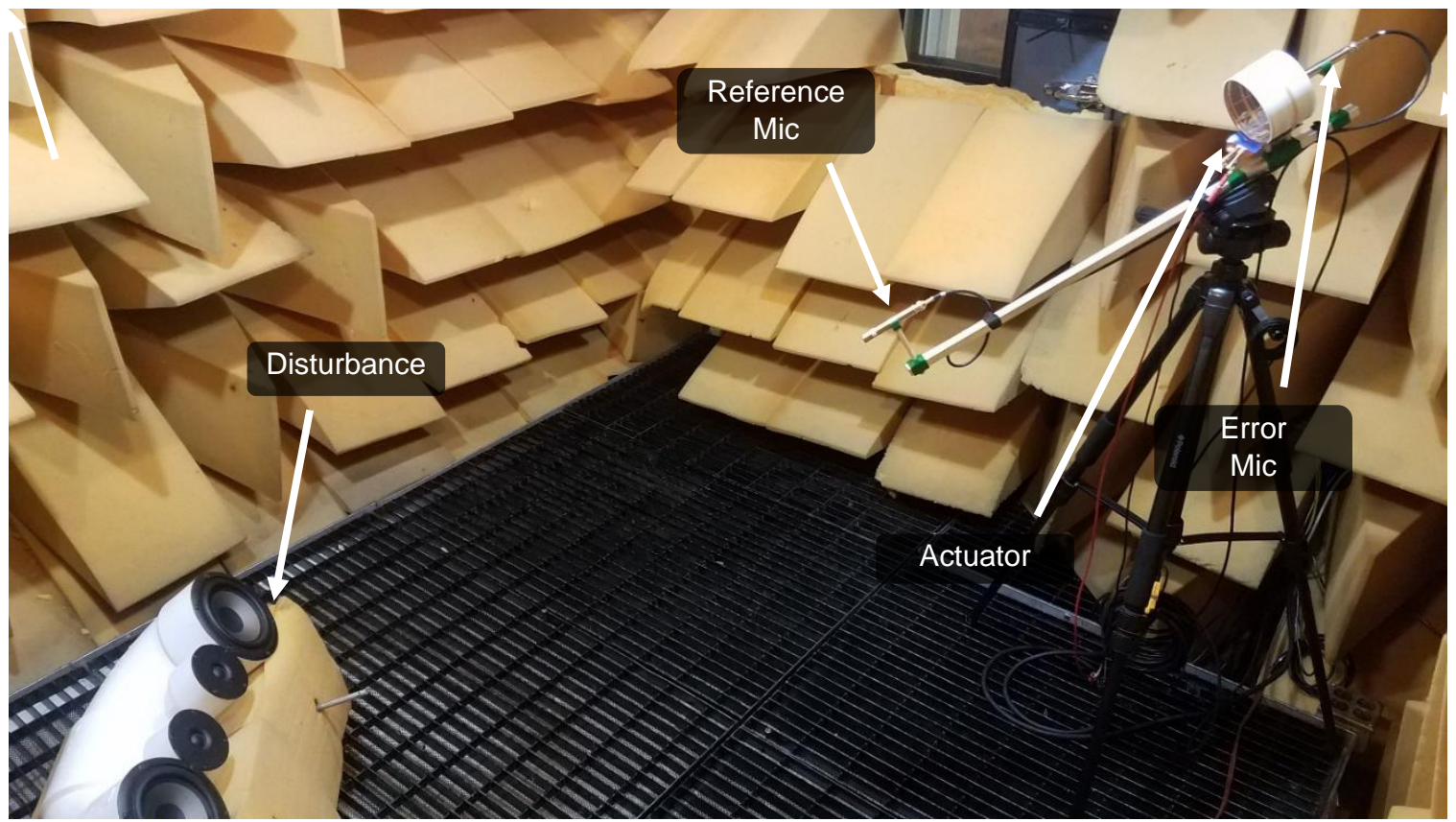

Figure 56: Microphone, Disturbance, and Actuator Configuration for Cancellation of Loudspeaker-Generated Noise in the Free Field

The first set of tests aimed at investigating system causality, so the reference microphone was moved incrementally towards the error microphone across trials (Figure 57). Broadband white noise between 200 and $2000 \mathrm{~Hz}$ was played from the disturbance and the thermophone was set to cancel noise at the error microphone using the Tiger WIFI-S controller. Distance between microphones was recorded alongside overall cancellation achieved (Figure 58 \& Figure 59).
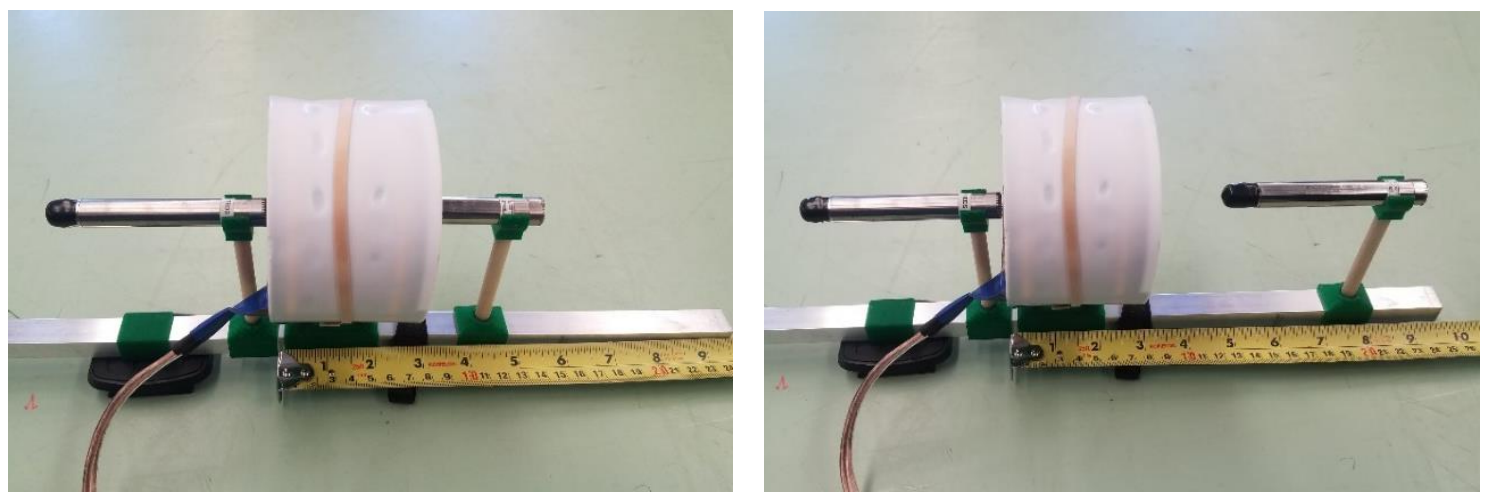

Figure 57: Example of Free-Field Cancellation Stand with Two Reference/Error Microphone Spacings (20 \& $12 \mathrm{~cm}$ ) 

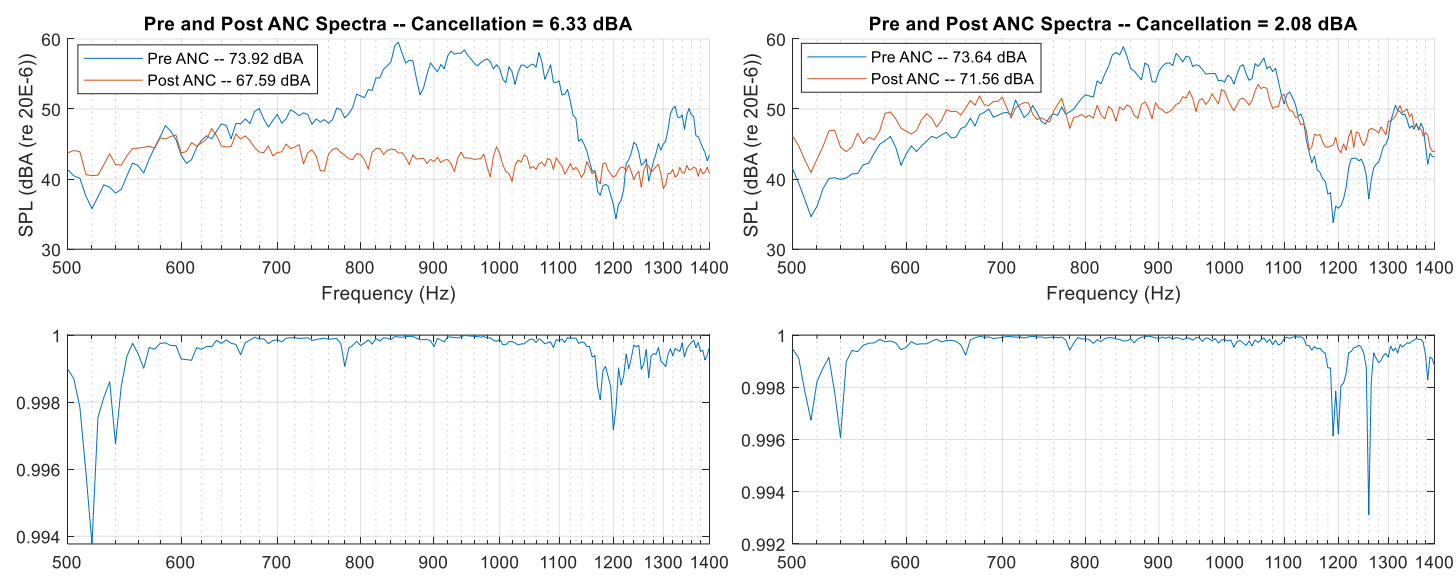

Figure 58: Example of Cancellation Levels at the Error Microphone for the Free-Field Cancellation Stand with For $20 \mathrm{~cm}$ (right) and $12 \mathrm{~cm}$ (left) Microphone Spacings

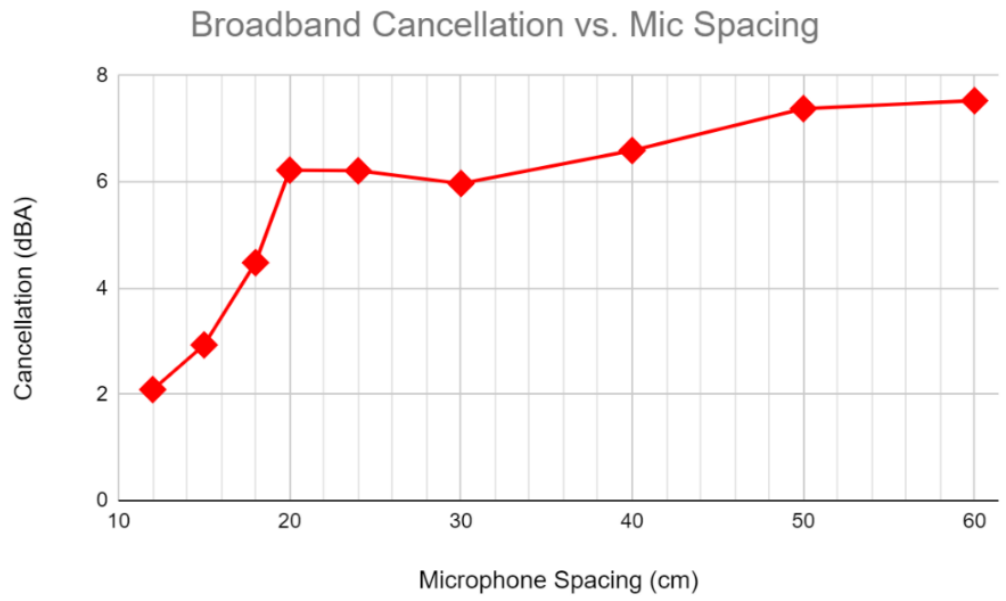

Figure 59: Cancellation Levels Achieved using the Free Field Cancellation Stand at Various Microphone Spacings - Loudspeaker Disturbance

Next, the free field cancellation stand was moved into a new setup where the blower fan in the partition-mount setup was used as the disturbance (Figure 60). Cancellation produced by the free-field cancellation stand at the error microphone location was once again measured. This was very similar to the testing performed in the free field Tiger WIFIS experimental suite - phases 02 and 03 , but with a different goal in mind. 


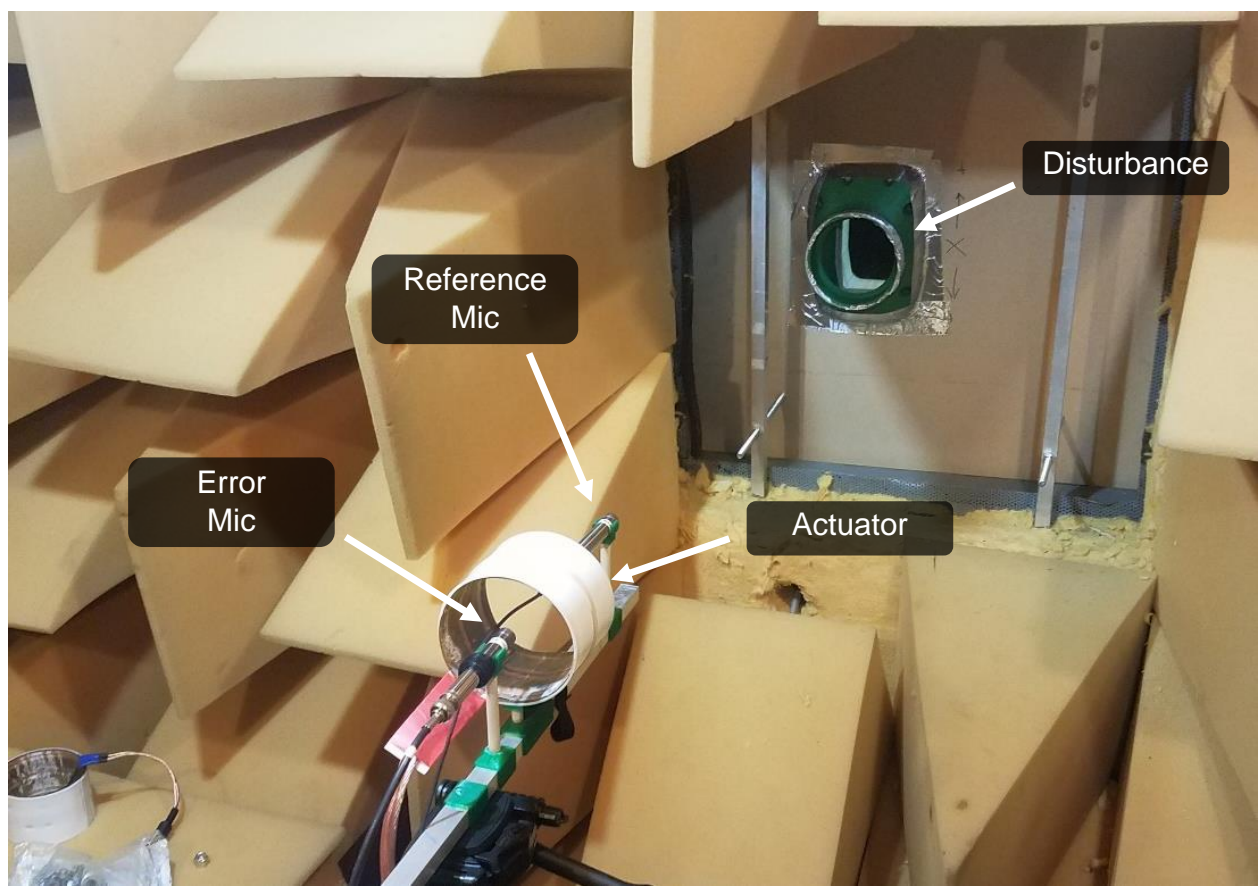

Figure 60: Free Field Cancellation Stand Placed to Cancel Noise from the Blower Fan in the Partition-Mount Configuration

Across trials, the free-field cancellation stand was moved incrementally into the airflow from the blower fan to reduce the coherence between the microphones (Figure 61).
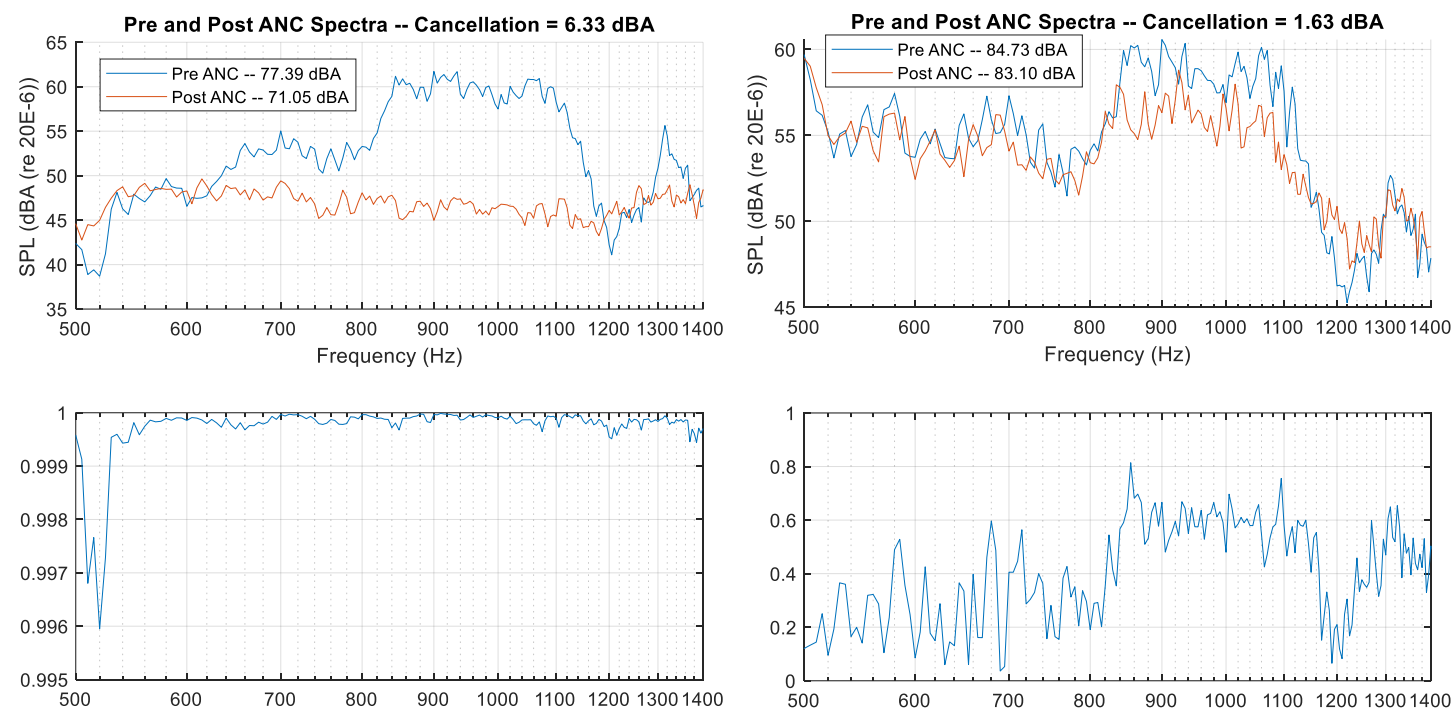

Figure 61: Noise Reduction Achieved with the Free Field Cancellation Stand for Two Trials with Different Levels of Microphone Coherence - Blower Fan Disturbance

Similar testing was performed using a ducted setup (Figure 62). White noise played through a loudspeaker installed on the blower fan (Figure 49) served as the disturbance noise, and the annular Teflon thermophone was installed in the ducting as the actuator. 
The error microphone was a $1 / 4 "$ microphone nearest the ducting outlet, and the reference microphone was provided by a surface-mount microphone inside the blower fan. The other $1 / 4$ " microphone in the ducting (typical reference transducer) was not used since it receives high levels of acoustic feedback from the actuator in a ducted setup.

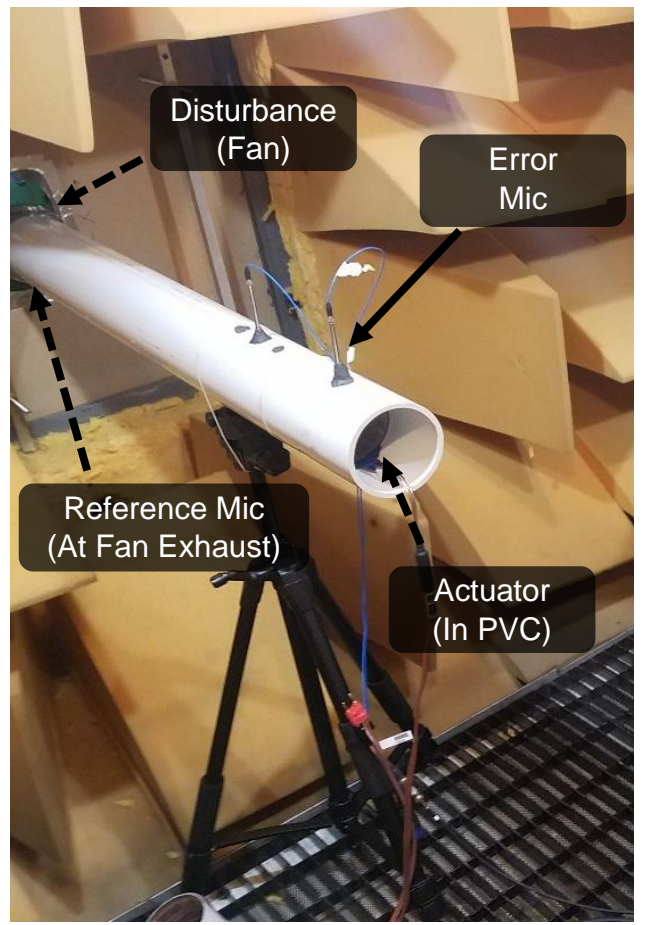

Figure 62: Microphone, Disturbance, and Actuator Configuration in a Ducted Setup for Coherence vs. Cancellation Experimental Trials

Coherence across the two microphones in the system was changed by increasing the speed of the blower fan, introducing airflow across the microphones (Figure 63).
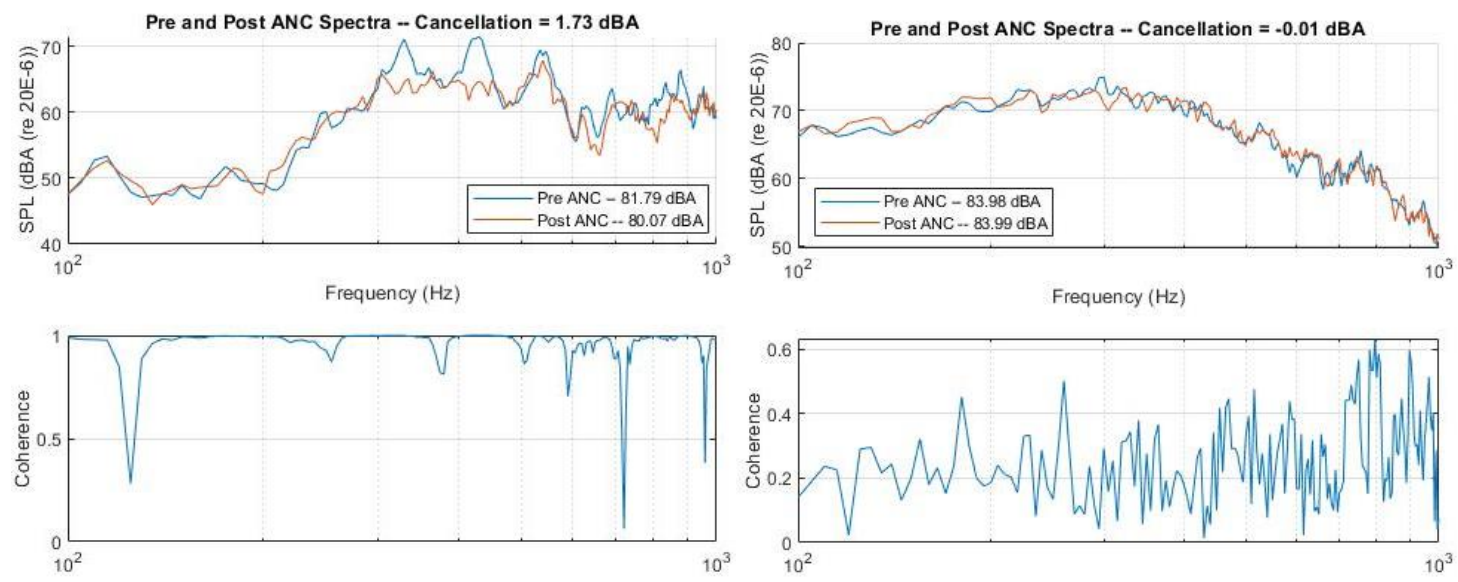

Figure 63: Best and Worst Case Results for Cancellation of White Noise in a Duct With Differing Levels of Airflow 


\subsection{In-Duct ANC Testing with The cRIO FPGA Controller}

This test suite aimed to measure the performance of an ANC system which aligns with the optimized model proposed later in this thesis. Because of this, it is very similar to the testing performed for the in-duct cancellation testing with the Tiger WIFI-S, the only major differences between the two suites being the test setups.

The setup for this suite once again used a 4" PVC pipe to simulate ducting in both the partition-mount and ANC demo configuration; various noises propagating through this ducting were subject to cancellation. Disturbances varied from trial to trial, being provided either by a loudspeaker at the end of the duct, by the blower fan itself, or by a combination of the two. Two $1 / 4$ " microphones served as reference and error transducers for these test trials. The microphones were flush-mounted with their front surfaces slightly recessed from the inside surface of the PVC pipe, and the microphone flush mount holes were then covered with a thin film of plastic tape to improve coherence (Figure 64).

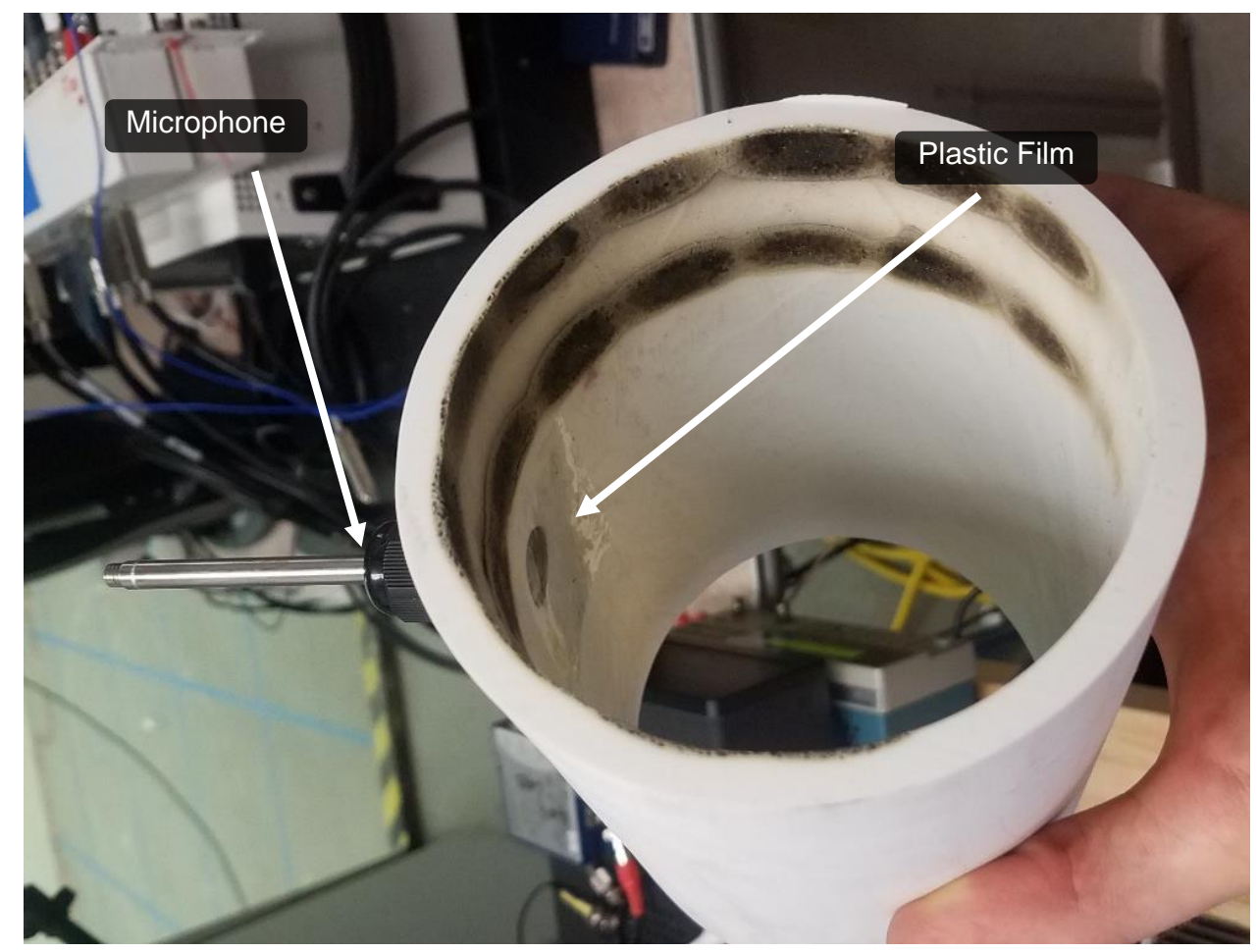

Figure 64: Plastic Film Covering the Microphone Flush Mount Hole to Improve Coherence

Two different sources served as the actuator for trials in this experiment. Initial tonal and broadband trials were performed with the Annular Aluminum Thermophone in the ANC Demo setup, but later trials were performed using the Annular Loudspeaker Array in the partition-mount setup (Figure 65). This switch was made to reduce the complexity of the system since a custom controller with new features was being tested. Finally, the custom cRIO FPGA controller ran the ANC algorithm for these test trials. 


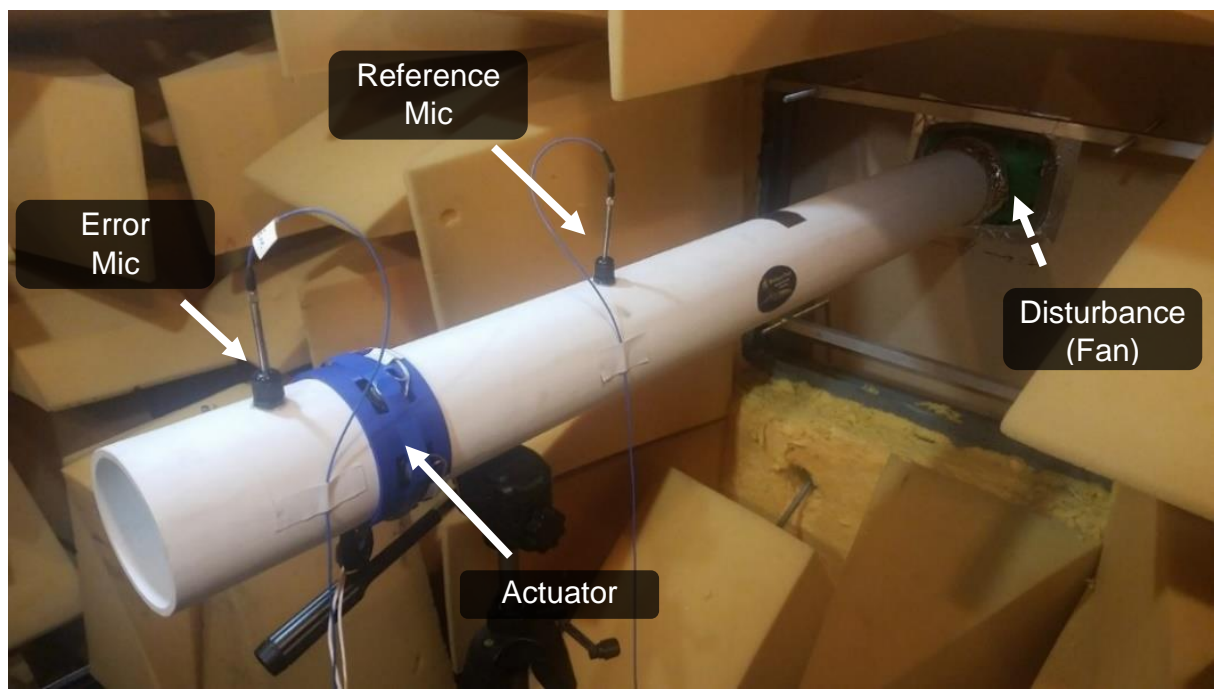

Figure 65: Microphone, Disturbance, and Actuator Configuration in the Ducted Partition-Mount Test Setup for Cancelling Noise with the Annular Loudspeaker Array

Different phases of this test suite can be organized according to the disturbance type. First, cancellation trials with tonal disturbances were attempted, then the disturbance was switched to broadband noise played from a loudspeaker and output from the fan, and finally, a tonal disturbance played over the broadband fan noise was used as the disturbance.

Tonal tests performed using the ANC demo configuration and the Annular Aluminum Thermophone showed good results. Single tone trials showed cancellation of up to 59.4 dBA overall, with $\sim 67 \mathrm{~dB}$ at the peak (Figure 66). Multitone experiments also yielded good cancellation results, with overall reductions of $\sim 21 \mathrm{dBA}$ possible (Figure 67 ).

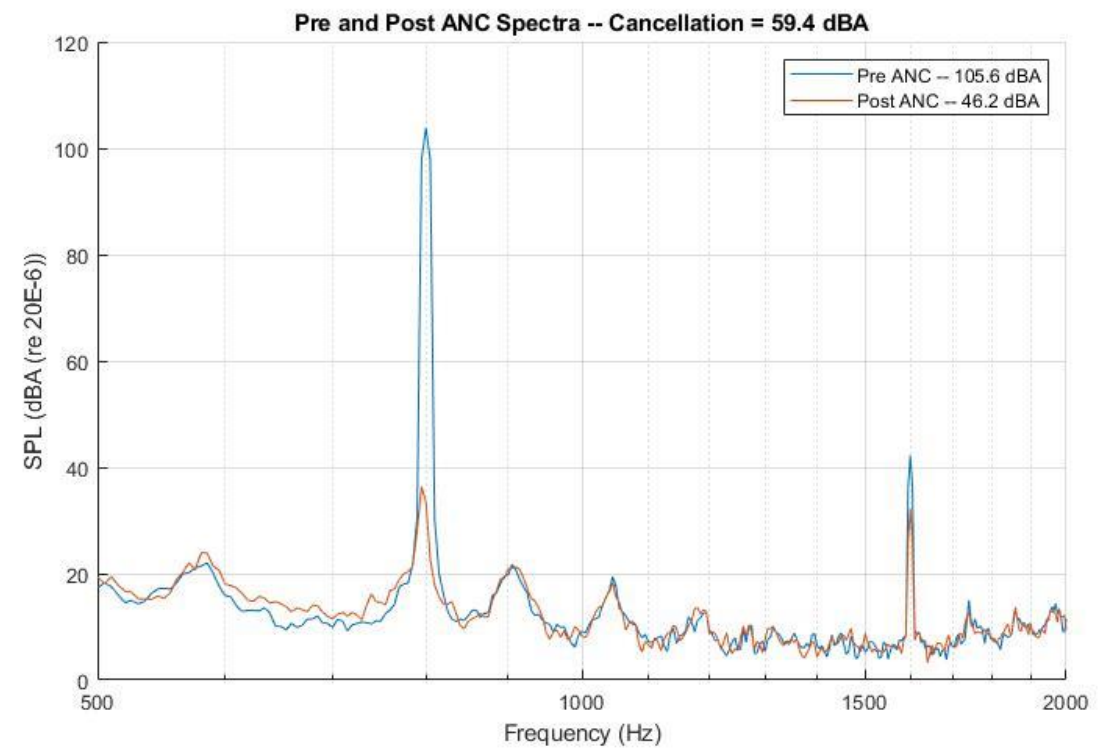

Figure 66: Cancelling a Tonal Disturbance with the cRIO FPGA Controller and the Annular Aluminum Thermophone Actuator 


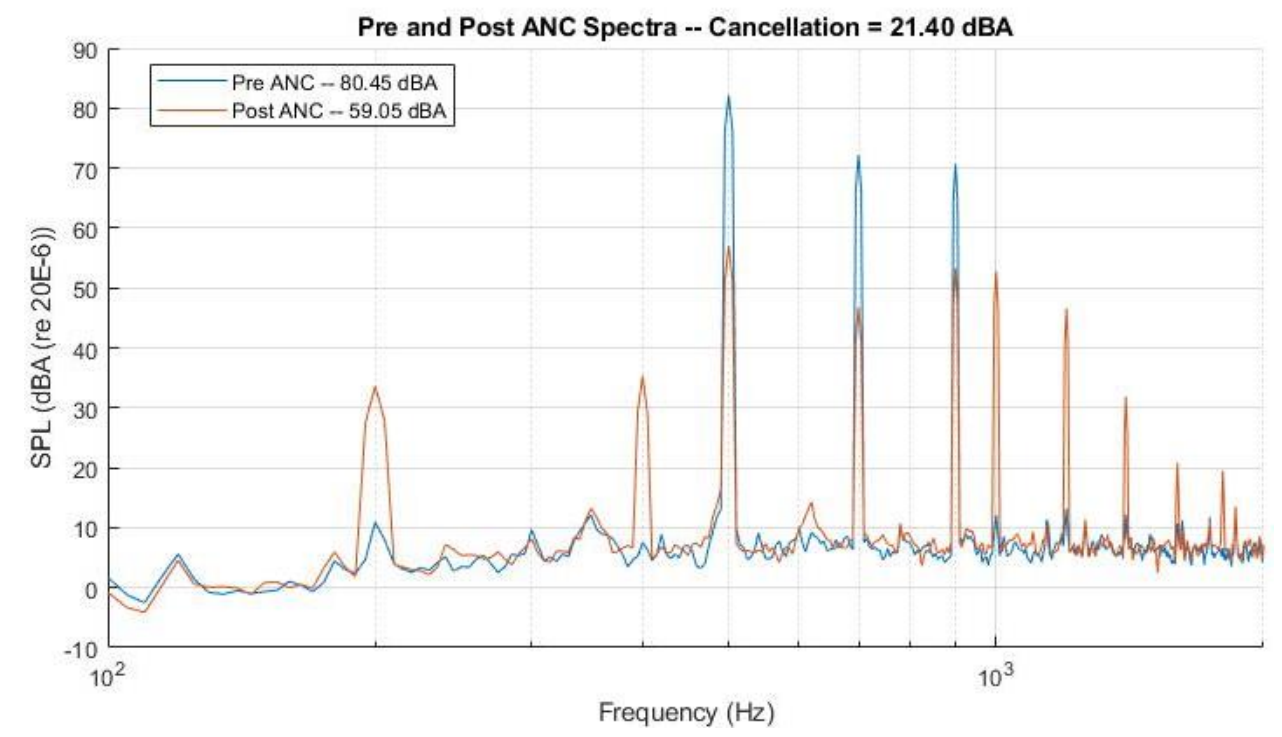

Figure 67: Cancelling a Tritone Disturbance with the cRIO FPGA Controller and the Annular Aluminum Thermophone Actuator

Broadband tests were also performed with the ANC demo configuration using the Annular Aluminum Thermophone. After some trial and error changes to the disturbance source done to maximize the overall broadband reduction possible, the excitation range of the disturbance was chosen to be $700-800 \mathrm{~Hz}$. An observant reader will note that the broadband disturbance used for this test is much narrower than in previous test; more comments on this will follow. Within this band the level was reduced anywhere between $\sim 4-16 \mathrm{dBA}$, resulting in an overall level change of 11.7dBA (Figure 68).

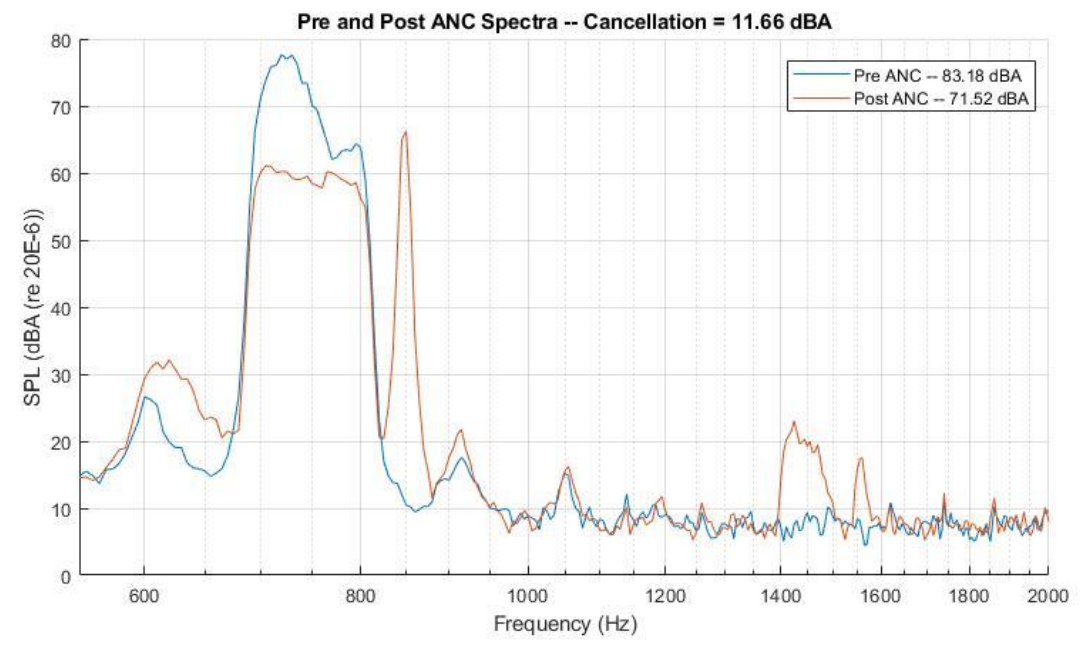

Figure 68: Cancellation of Broadband Noise with the cRIO FPGA Controller and the Annular Aluminum Thermophone Actuator

After tonal and broadband tests were performed using the ANC demo configuration, the test setup was swapped to the partition-mount configuration with the Annular Loudspeaker Array serving as reference transducer. 
The first trial in this configuration was the cancellation of an $800 \mathrm{~Hz}$ tone played through the loudspeaker installed in the fan. Since the tonal performance of the controller had already been demonstrated, this was mainly done as a formality to ensure that the dynamic step size feature of the controller, and the Annular Loudspeaker Array were functioning properly. Cancellation achieved in this configuration was $\sim 36 \mathrm{dBA}$ (Figure 69 ).

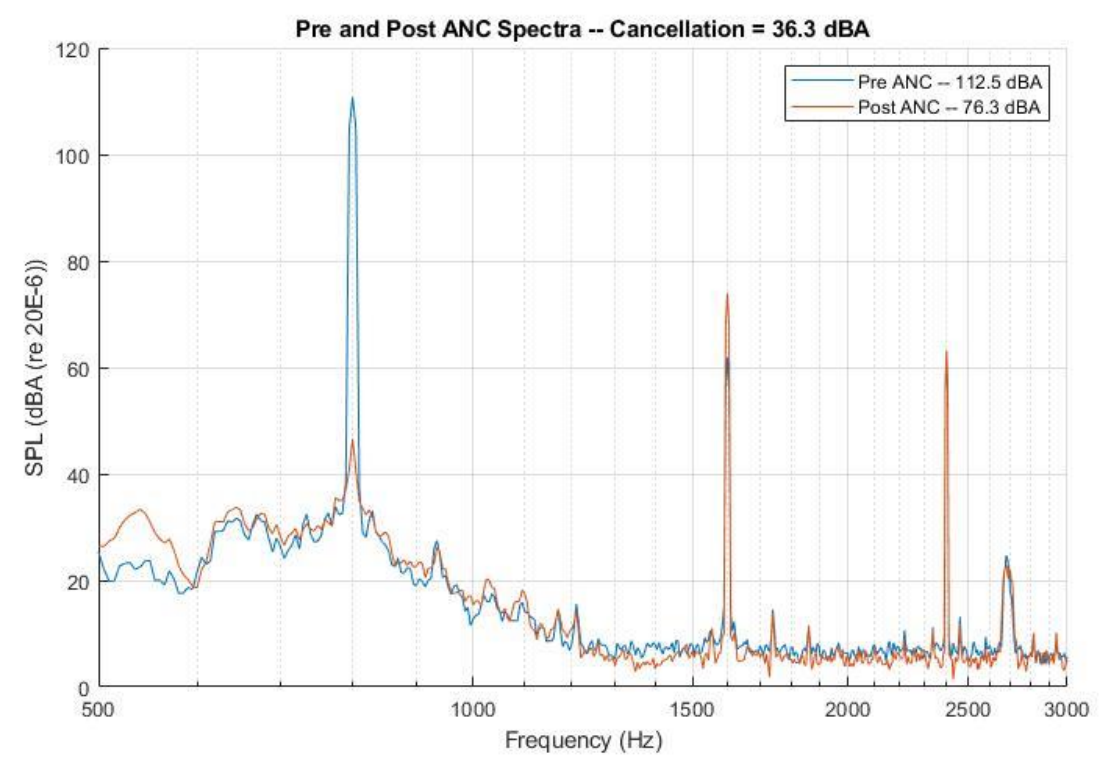

Figure 69: Cancellation of an $800 \mathrm{~Hz}$ Tone Using the Dynamic Step Size Feature of the cRIO FPGA Controller and the Annular Aluminum Thermophone Actuator

Curiosity about the narrow frequency limits required for successful broadband cancellation using the thermophones (Figure 68) prompted more broadband testing in the new configuration as comparison of results across test setups could possibly yield useful information. A wider band was used in this case; the excitation range of the white noise disturbance used was increased to $850-1500 \mathrm{~Hz}$. Cancellation results were found to be small relative to expectations, with an overall level reduction of only $3.8 \mathrm{dBA}$ when a microphone was used as the reference transducer (Figure 70). 


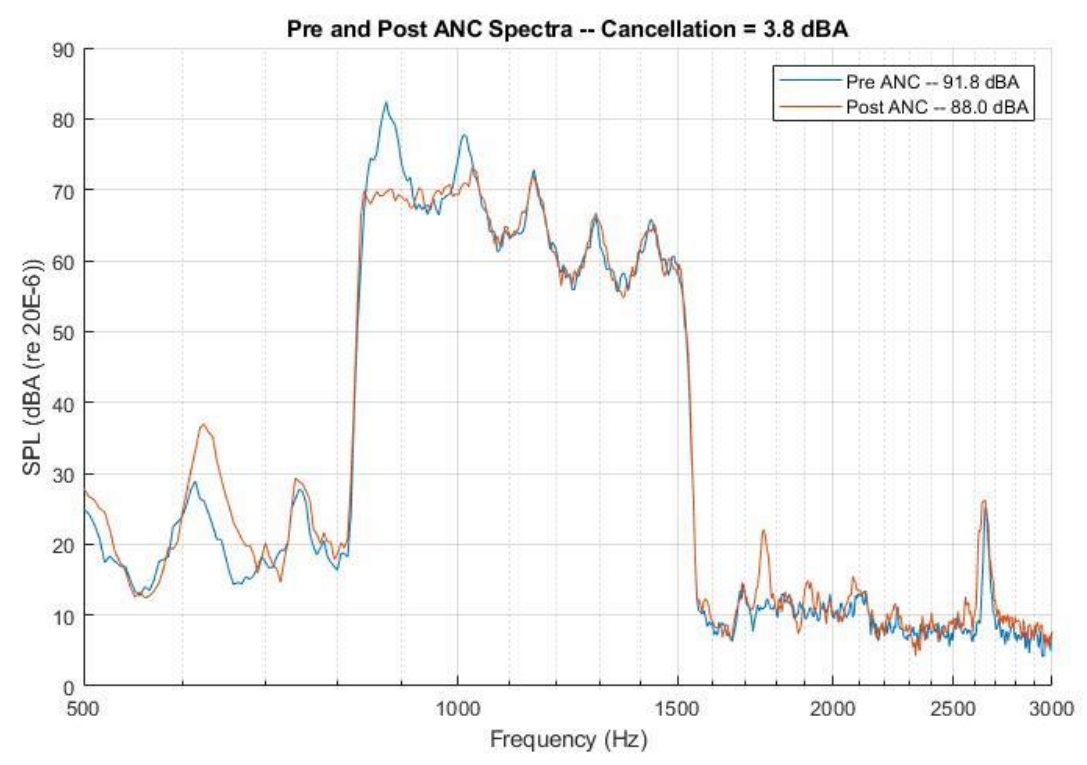

Figure 70: Cancellation of a Broadband Disturbance Using the cRIO FPGA Controller with the Annular Loudspeaker Array Actuator and a Microphone Providing the Reference

Another trial was run with a nearly identical disturbance and setup, the one exception being a change in the acquisition of the reference signal. As in previous testing, in this trial the reference signal input was connected directly to the disturbance signal. In this configuration the overall cancellation levels were increased significantly over the last test, with the reduction being as high as $14.4 \mathrm{dBA}$ (Figure 71).

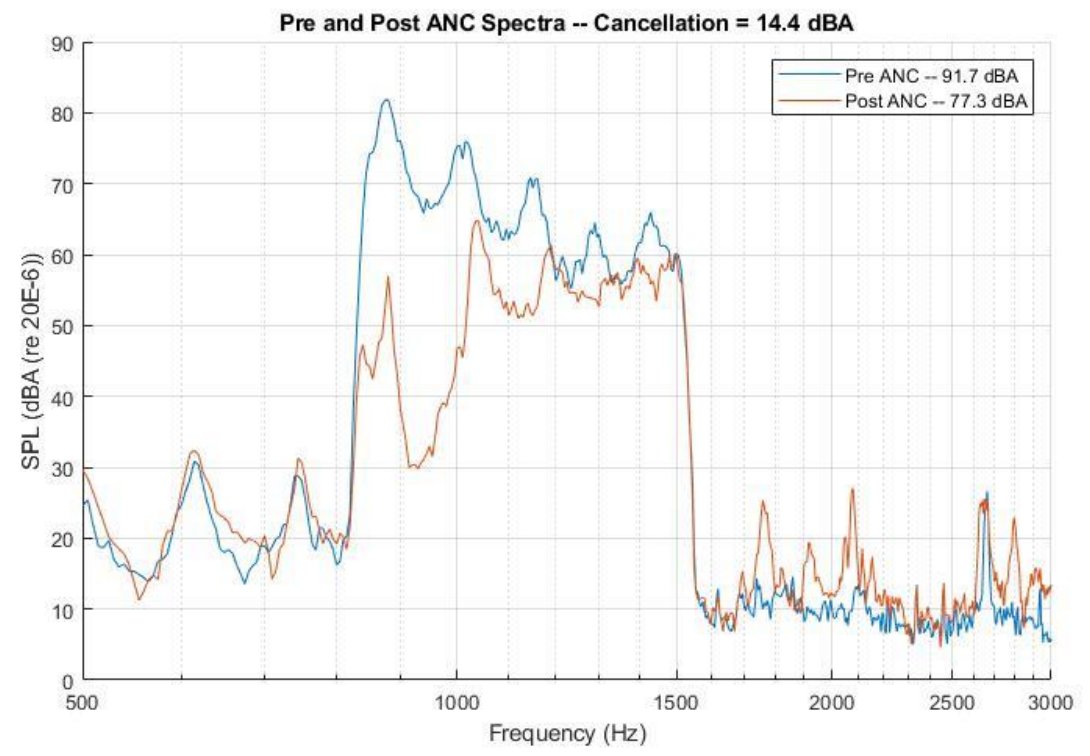

Figure 71: Cancellation of a Broadband Disturbance Using the cRIO FPGA Controller with the Annular Loudspeaker Array Actuator and a Reference Signal Acquired Directly from the Loudspeaker Disturbance 
In order to test another broadband disturbance which does not suffer from the presence of pipe modes in the spectra, fan noise cancellation was attempted in the duct. Multiple trials were attempted, but no cancellation was possible in any case. Spectra at the error microphone location were recorded before and after the application of ANC, along with the coherence between the error and reference microphone (Figure 72).
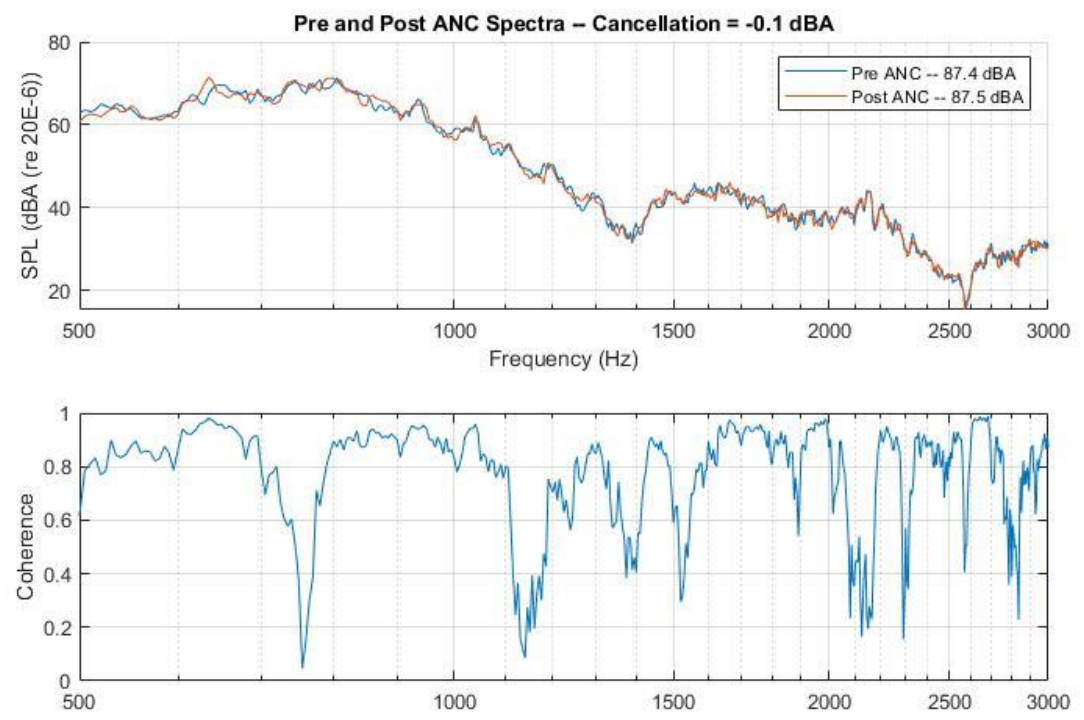

Figure 72: Spectra at the Error Microphone Location Before and After the Application of ANC using the cRIO FPGA Controller and the Annular Loudspeaker Array Actuator -- Coherence Between Error and Reference Microphones Before Application of ANC

Finally, the last trials run in the partition-mount configuration attempted to cancel a tonal disturbance which was played over the fan noise. In these trials the tone was completely eliminated, which led to high overall level reductions, but it should be noted that broadband noise was not affected. 


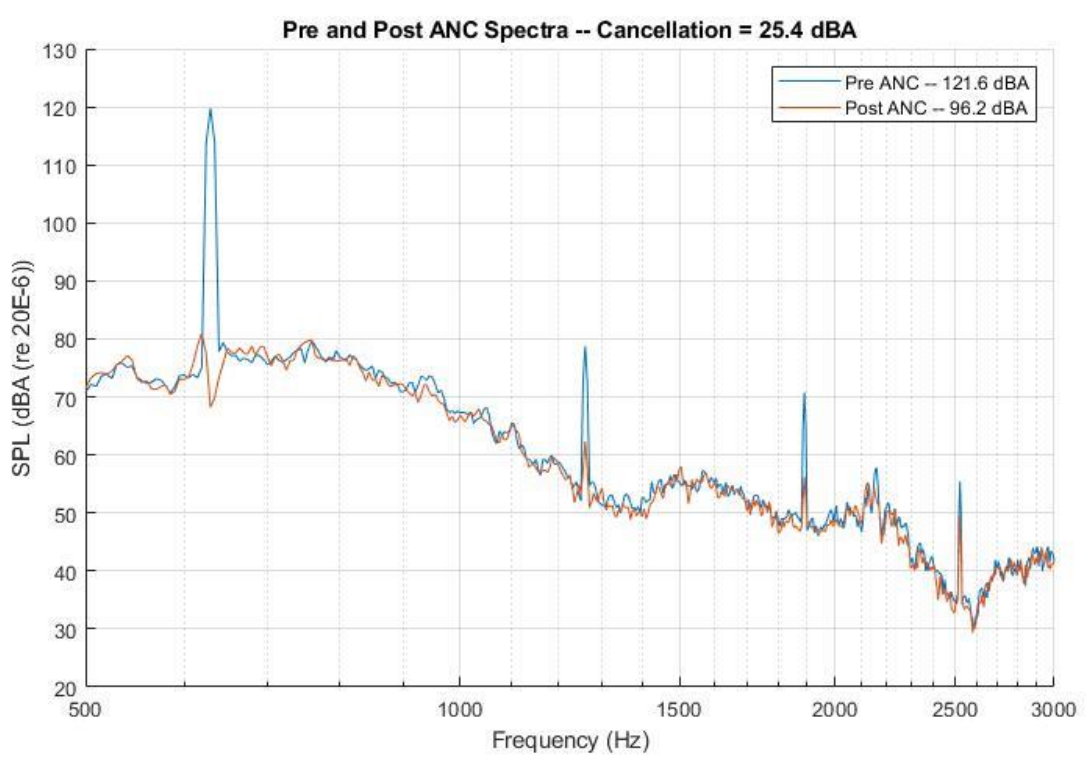

Figure 73: Cancellation of a Tonal Disturbance Played Over Fan Noise with the CRIO FPGA Controller \& the Annular Loudspeaker Array Actuator 


\section{Development of an FPGA-Based ANC Controller}

\subsection{Motivation \& Overview}

The inclusion of this section without requiring some circular navigation on the reader's part is unavoidable, so it is placed here. To more fully understand the motivation behind this controller it would be useful to read the first few bits of the Testing Results and Interpretations Section. This lays out in detail the findings from early testing that led to the development of a custom controller; they will be summarized in brief here.

Before June 2020 all of the ANC testing done in this case study was performed with either the GLSV Order-Based controller or the Tiger WIFI-S controller. Throughout the testing and system development many test setup specific factors that limited ANC performance were found. Ultimately, trends across these factors were analyzed and a generalized set of system characteristics limiting performance was theorized. Two of these characteristics, causality, and coherence, were potentially solvable using hardware already owned by Michigan Tech's Dynamic Systems Lab. Acoustic feedback neutralization, however, was not.

Simply put, acoustic feedback happens when the output of the ANC system's control loudspeaker is measured at the reference input of the system. This creates a feedback loop which can impact system performance or cause system instability. A typical strategy for remedying this is as follows: the response of the control loudspeaker at the reference input location is calculated in real time and removed from the incoming reference signal. This is a task which needs to be done digitally by the controller using an extra filtering step in the ANC algorithm. Since neither the GLSV controller nor the Tiger WIFI-S sport this feature in their algorithms, the decision was made to develop a new controller which would be capable of this.

\subsection{Background of Algorithm}

As was stated in the ANC primer section of this thesis, many ANC algorithms exist [30, $36,37]$. For this controller, the time-domain FXLMS algorithm was chosen due to its simplicity and readily available documentation. Specifically, the time-domain FXLMS algorithm with feedback neutralization was used (Figure 74) [38-40]. At its core, the timedomain FXLMS algorithm is an iterative gradient descent algorithm. Using an adaptive filter, a reference signal is used to synthesize a control signal which interacts destructively with unwanted noise. A relationship between the vector representing the taps of this adaptive filter and the signal from an error transducer is formulated. This relationship is commonly represented as a "surface" called the error surface. This surface exists in a vector space whose number of dimensions is equal to the number of taps in the adaptive filter. The gradient of this surface is then calculated, and with every iteration of the algorithm the adaptive filter tap vector "moves" along the surface in a direction opposite to the gradient of the surface. In this way, the filter tap vector eventually converges to the location which produces the minimum error. Since the error signal represents acoustic 
measurements at a microphone, this results in the sound being controlled at this location. A brief derivation of this algorithm is laid out here which relies on basic information about generalized ANC systems. For an explanation of the basic components of an ANC system, see the introduction.

\section{Time Domain FXLMS With Feedback Neutralization}

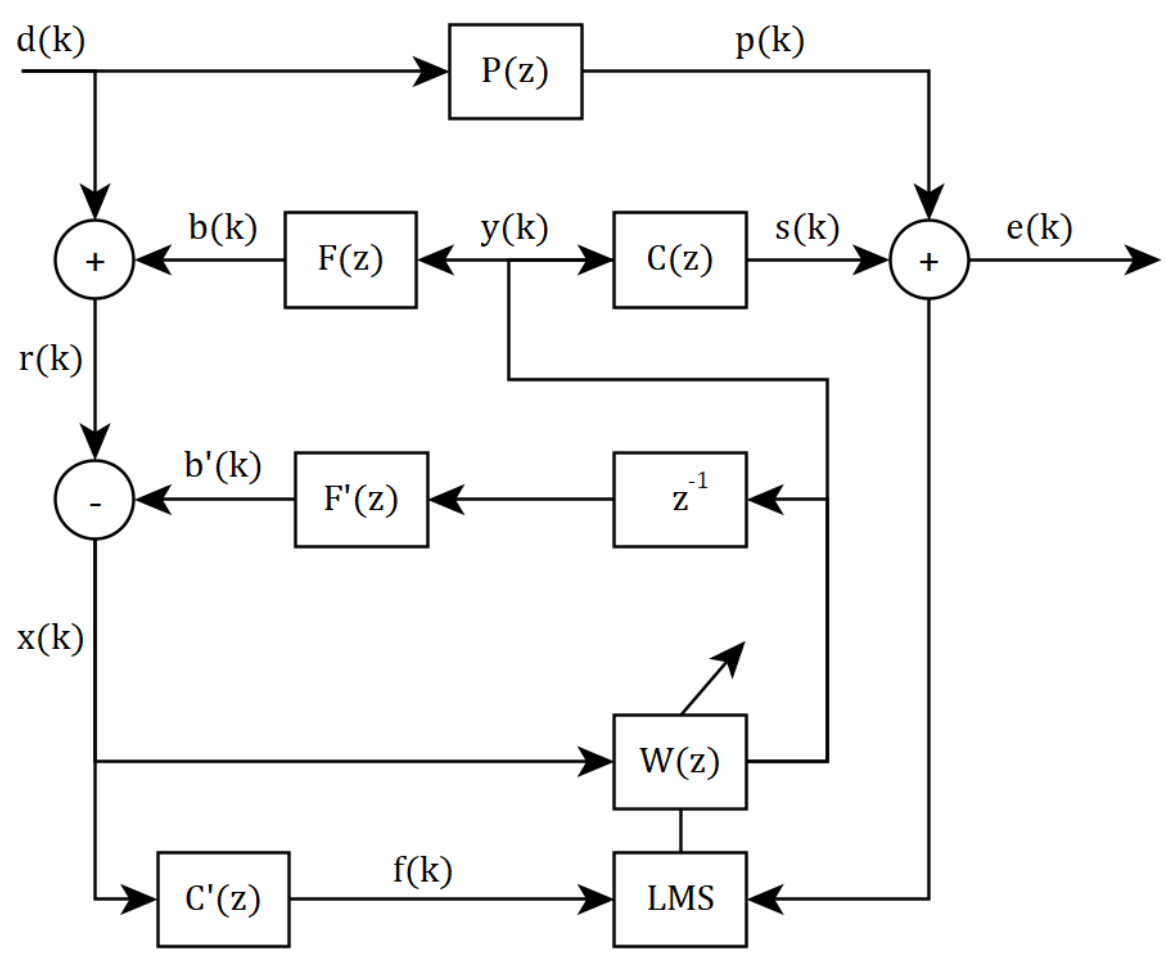

Figure 74: Time-Domain FXLMS Algorithm with Feedback Neutralization

Consider the above flowchart, starting with the disturbance noise, $d(k)$, the primary noise which is to be cancelled at time $k$. In the physical world it travels through the ANC system to the error transducer, being subject to the transfer function $P(z)$, the primary path, between reference and error transducer locations. At the reference transducer location samples are taken which include contributions from not only the disturbance noise, but from acoustic feedback noise as well. This feedback propagates back through the physical system from the actuator, experiencing transfer function $F(z)$, the feedback path, to become $b(k)$. The combination of the primary noise and the feedback noise is $r(k)$, the signal measured at the reference location. A digital estimate of the contribution from acoustic feedback, $b^{\prime}(k)$, is calculated using the delayed control signal $y(k-1)$, and an estimate of the feedback path, $F^{\prime}(z)$. The result of this calculation is then subtracted from $r(k)$ to produce signal $x(k)$, an estimate of the disturbance noise at the reference transducer location in the absence of acoustic feedback. This signal is then used as the input to the FXLMS filter, $W(z)$, to produce the control signal $y(k)$ which is played as noise through the actuator. As the control noise travels through the physical system from the 
actuator to the error transducer it is subject to transfer function $C(z)$, the secondary path. Finally, a sample at the error transducer is taken, $e(k)$, which contains contributions from both the disturbance noise at the error location, $p(k)$, and the control noise at the error location, $s(k)$. Depending on $W(z)$, these contributions may interact differently, obviously with destructive interference representing the desired result.

If the optimal control filter taps for achieving this result were known ahead of time, this would be all that is required for noise cancellation. The ability to adaptively change control filter behavior to accommodate different disturbance noises and changing environments, however, is of great importance. The signal $x(k)$ is filtered through the estimate of the secondary path transfer function, $C^{\prime}(z)$ to produce signal $f(k)$. Within the vector space containing the error surface, $f(k)$ points in the direction of the gradient of the error surface. It is used to update the filter, being scaled and then subtracted from the current filter tap vector in order to produce a new $W(z)$ using the following equation:

$$
\boldsymbol{W}(z)(k+1)=\alpha * \boldsymbol{W}(z)(k)-\mu * e(k) * \boldsymbol{f}(k) \quad \text { Eqn.6 }
$$

Where $\alpha$ and $\mu$ are the leakage factor, and the step size, respectively. The step size determines how far the filter tap vector will "move" along the gradient surface. In general, using a larger step size will mean that the algorithm converges more quickly, but a smaller step size will result in the convergence being closer to the ideal solution. The leakage factor is some coefficient between 0 and 1 that is used to limit the magnitude of the filter tap vector; the magnitude of the filter taps is reduced slightly with every iteration of the update equation. This is especially important in a digital environment where the precision of computations is limited.

At this point a short discussion about the effects of digitization of the above algorithm is necessary. In an analog world, the signals and transfer functions from Figure 74 are continuous, and the relationships between them takes physical or analytical form. To run on a computer, however, this must be digitized. In doing so, everything is converted to discrete representations using vectors and linear algebra. Going forward (as well as in Eqn. 06), signals at time $k$ and filters are represented by a vector ${ }^{4}$ when written in bold, and by their most recent discrete value otherwise.

\subsection{Hardware and Implementation Details}

\subsubsection{Hardware Used}

Because it was already owned by the Michigan Tech's Dynamic Systems Lab, a National Instruments cRIO 9031 real-time / FPGA embedded controller was chosen for use as the ANC controller. This controller features a Kintex-7 70T FPGA, which runs the FXLMS algorithm, as well as a $1.33 \mathrm{GHz}$ dual-core real-time processor which monitors and controls the algorithm. The I/O cards used in the controller were the NI 9234 for analog input, and the NI 9260 for analog output.

\footnotetext{
${ }^{4}$ In the case of signal, the vector contains the $n$ most recent discrete values of the signal
} 


\subsubsection{FPGA Implementation}

\subsubsection{Bridging the Gap: Flowchart to Fixed-Point}

Studying the time-domain FXLMS algorithm will reveal that almost all the mathematical operations it contains are digital filtering operations, the one exception being the updating of the FXLMS filter coefficients. In the time domain, digital filtering is mathematically equivalent to convolution [41]. In post-processing, convolution can produce an entire output signal in one go, requiring the calculation of many successive dot products to produce samples which make up a complete time trace. In real-time, however, dot products between a signal delay chain vector and a filter tap vector are calculated pointby-point every time a new sample is introduced to the delay chain. The result of these individual calculations is the newest sample in the digitally filtered output signal.

$$
y(k)=\sum_{i=0}^{n} \boldsymbol{x}(i)(k) * \boldsymbol{W}(i)(k)
$$

To implement the FXLMS algorithm, three filter tap vectors and three signal delay chain vectors are used:

- $\boldsymbol{F}^{\prime}(z)$ : $\quad$ Feedback Path Filter Taps

- $\boldsymbol{C}^{\prime}(z)$ : Cancellation Path Filter Taps

- $\boldsymbol{W}(z)$ : FXLMS Filter Taps

- $\boldsymbol{x}(k)$ : Reference Signal Delay Chain

- $\boldsymbol{f}(k)$ : Filtered-X Signal Delay Chain

- $\boldsymbol{y}(k)$ : Control Signal Delay Chain

Each time a new sample, $r(k)$, arrives from the analog to digital converter (ADC), several dot product operations are performed sequentially, the end result being the generation of a new output sample, $y(k)$, which is sent to the actuator (Figure 75): 


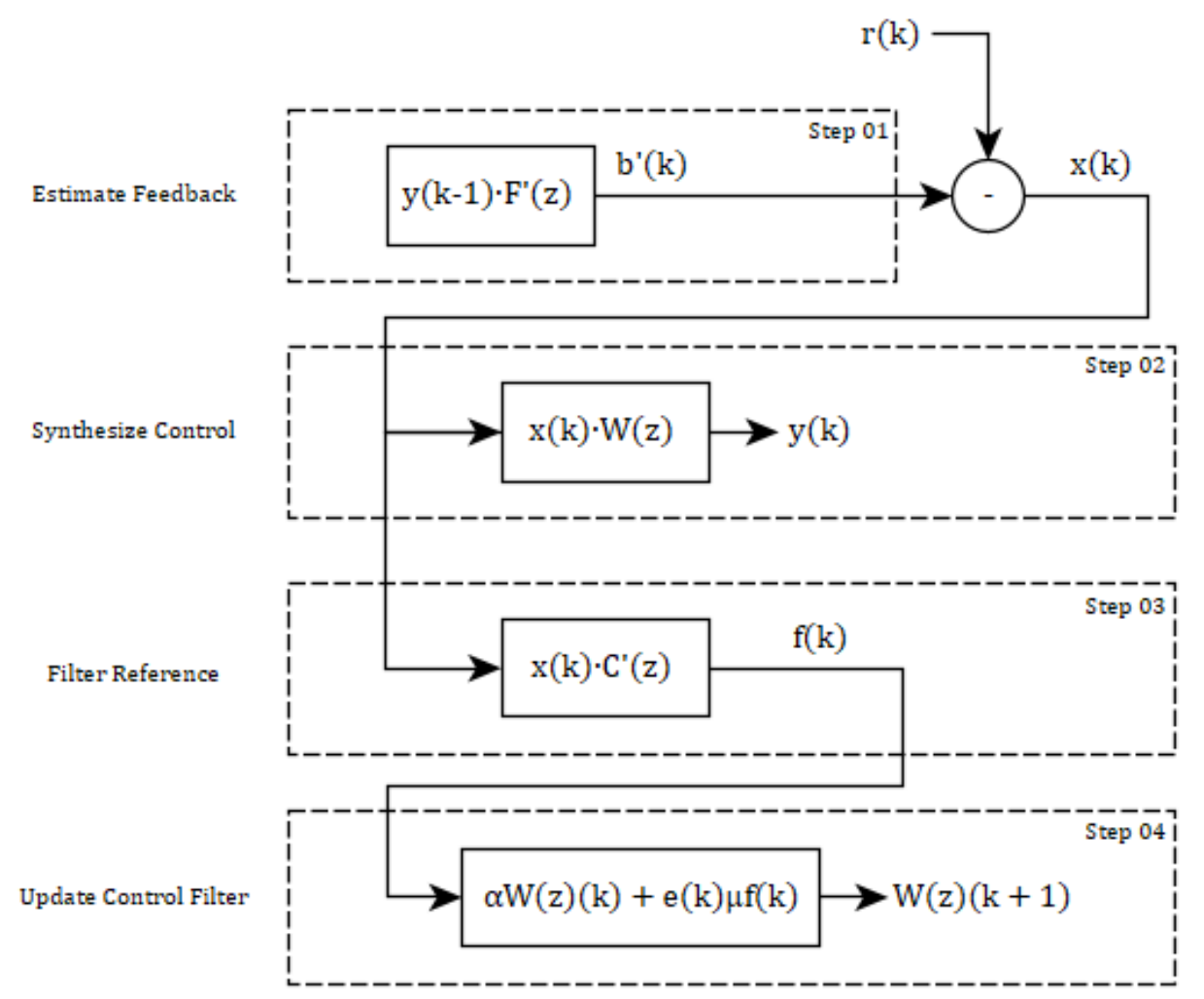

Figure 75: Sequential Filtering and Filter Update Operations of the Time-Domain FXLMS Algorithm

As can be seen above, the result of one dot product operation is used as the newest sample in the signal delay chain required for the next dot product operation. In addition to synthesizing a new output sample, the FXLMS filter tap vector must also be updated every time a new sample is taken using (Eqn. 06). Ideally, all dot product operations and the filter update operation should be finished before the next sample is read from the ADC. By doing this the controller only takes one sample to respond to an input, allowing for the minimum microphone spacing ${ }^{5}$ whilst still maintaining system causality. This is of great importance for broadband control.

Because the inner workings of the FXLMS algorithm rely so heavily digital filtering operations, the core of the architecture in this controller is built around executing them efficiently. This can be broken down further into two separate tasks: storage and reading/writing of vectors (signal delay chains and filter taps), and performing elementwise math on these vectors. All signal delay chain and filter tap vectors have their own unique locations in block random-access memory (BRAM), but there is only one central mathematical engine which processes them, performing either a dot product operation or a filter update operation when called. Throughout the execution of the algorithm different vector pairs are used sequentially as inputs to this engine ${ }^{6}$. By using one centralized

${ }^{5}$ Minimum microphone spacing will be inversely proportional to sampling frequency in this scheme

${ }^{6}$ In Figure 75, the four "steps" represent operations performed by the mathematical engine. 
engine, the fixed-point precision of the algorithm could be greatly increased, albeit at the cost of potential computation speed. The algorithm retains an acceptably high speed, however, thanks to parallel implementation of operations performed by the mathematical engine. For any such operation, all vector read/write and multiplication/addition operations happen 16 at a time, meaning that the number of processor ticks required to calculate a result is equal to $1 / 16^{\text {th }}$ the number of elements in the vector plus a small fixed number representing pipelining windup.

\subsubsection{Storage and Read/Write Strategies for Signal Delay Chain and Filter Tap Vectors}

As stated before, filter tap / signal delay chain vectors are stored in BRAM on the FPGA. Elements of all vectors are configured as signed fixed-point numbers having 47 bits and four integer bits $< \pm 47,4>$. In order to be accessed in parallel, elements of each vector are distributed across a group of 16 separately configured BRAM blocks, each block having a size of 256 elements. Hence, each vector contains 4096 elements. A 12-bit fixed point number, called the spatial address ${ }^{7}$ since it identifies the physical location in the memory, serves as the address for individual elements within a group. The spatial address can be broken down further into its first four bits, the group-scoped bits, and its last 8 bits, the block-scoped bits.

The group-scoped address identifies a BRAM block in the group while the block-scoped address identifies the position within that block. VI's were developed for each vector to read all 16 elements which share identical block-scoped bits in parallel. When two vectors are used as inputs to the math engine, their elements are retrieved 16 at a time in this fashion (Figure 76).

${ }^{7}$ Don't let the terms group, block, and spatial address get away from you, they are important going forward. 


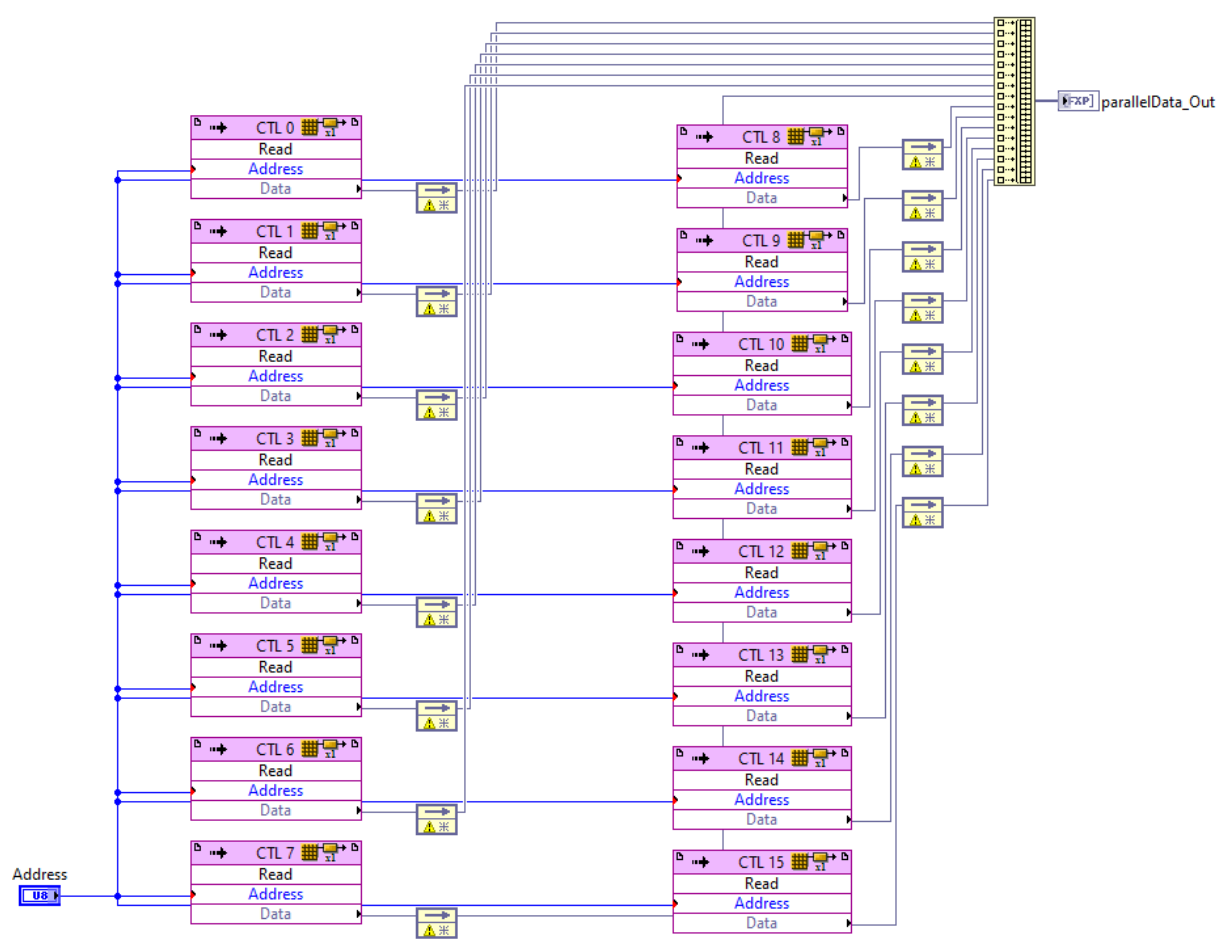

Figure 76: 16 Elements Sharing Block-Scoped Addresses are Read from a Vector

At this point an important distinction between the memory structure of filter tap vectors and signal delay chain vectors must be made. Filter tap vectors are either not updated during the execution of the algorithm, as is the case for the cancellation and feedback paths, or have all of their elements updated simultaneously as is the case with the FXLMS coefficients. Signal delay chains, however, must act as a buffer whose elements correspond to different discrete delays. When a signal delay chain is updated, three things happen: the oldest sample is removed from the vector, individual elements must have their corresponding delay in the signal chain changed, and the newest sample is added to the vector.

To do this efficiently, these groups are configured as circular buffers; all three of these steps can be achieved in one operation in this case [42]. The first time a sample is written to a signal delay chain it is written to address number 4095 . The write address of the next sample is then 4094, and the following sample's write address is 4093 etc. The write address continues to decrement until it reaches zero at which point it loops back to 4095, provided the carry out bit of the bitwise subtractor which indexes the write address is ignored (Figure 77). 


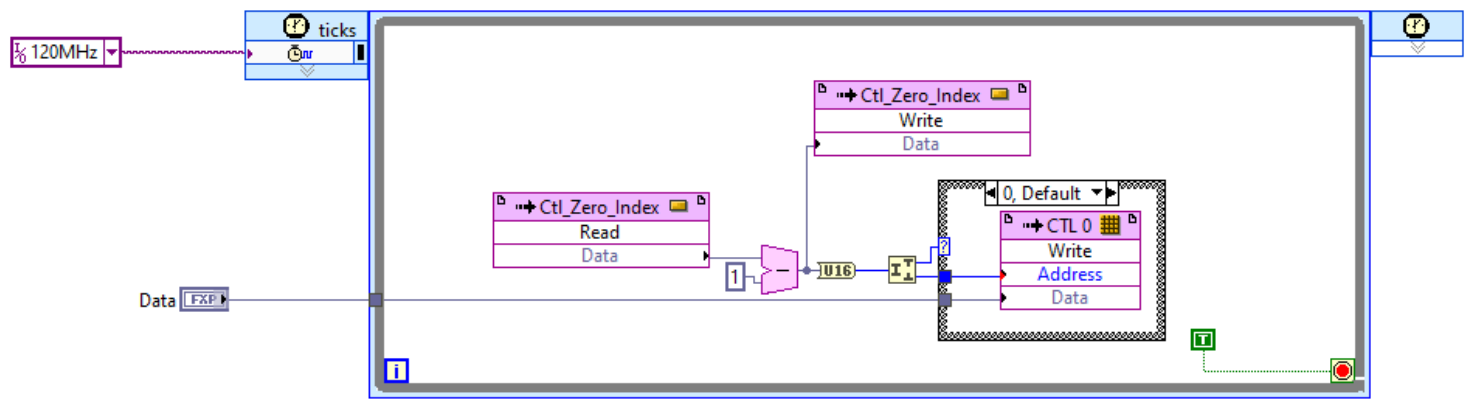

Figure 77: Writing A Single Element to a Circular Buffer and Incrementing the Write Address

One drawback of using a circular buffer is that the spatial address of the most recent sample in the signal delay chain is constantly changing. It is important to distinguish between this spatial address and a new address called the temporal address, which represents the current delay of any given element. The most recent sample has a temporal address of 0 and the least-recent sample has a temporal address of 4095 . For filter tap vectors, the spatial and temporal addresses will always be equal, but this is not the case for signal delay chain vectors. To ensure that datapoints read from both vectors have identical temporal addresses, as is required for real time convolution, the block-scoped bits of the read address sent to the filter tap vector must be modified before they can be used to read from the corresponding signal delay chain vector. Luckily, this modification is simple and can be achieved using a simple eight-bit adder.

$$
l_{s d}=l_{f t}+o
$$

Where $l_{s d}$ are the block-scoped bits from the desired read spatial address of the signal delay chain vector, $l_{f t}$ are the block-scoped bits from the read spatial address of the filter tap vector, and $o$ are the block-scoped bits from the most recent write address of the signal delay chain vector. This calculation must occur with wrapping about 255; the most efficient way to do this is to use a bitwise adder and to ignore the carry bit (Figure 78).

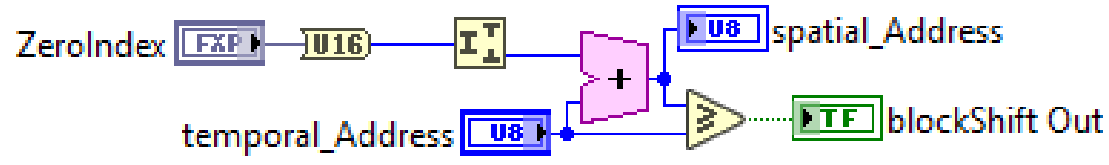

\section{Figure 78: Converting the Block-Scoped Temporal Address into the Block-Scoped Spatial Address}

Sixteen elements from each vector have been retrieved, and it has been shown that the block-scoped bits of their temporal addresses all match. One further alignment step may be required, however. It is not guaranteed that the temporal addresses are aligned at the group-scoped level. Data from the first block within the signal delay chain group may not align with data from the first block within the filter tap group. To remove this misalignment the 16 elements retrieved from the signal delay chain vector must be rotated by one of two numbers, depending on whether or not wrapping occurred at the adder in the blockscoped alignment step (Figure 78). If no wrapping occurred, the array is rotated by a 
number equaling the group-scoped portion of the circular buffer's most recent write address. If wrapping did occur, the array is rotated by this same amount plus one (Figure 79).

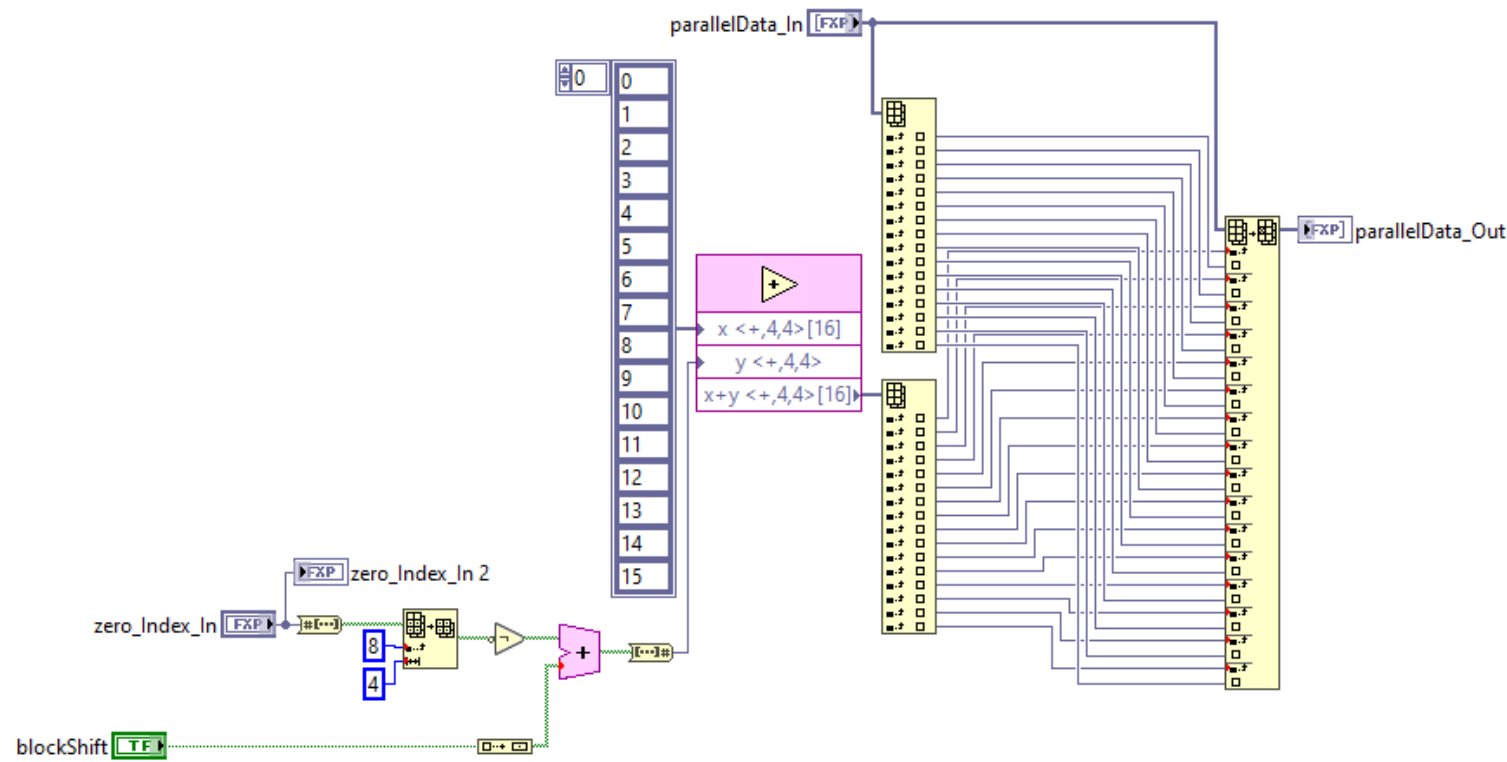

Figure 79: Rearranging the 16 Elements Retrieved from a Circular Buffer for Group-Scoped Temporal Alignment

All these steps are performed within a single-cycle timed loop such that 16 temporally aligned datapoints from two vectors can be retrieved every clock cycle, with a latency of four clock ticks. These datapoints are then ready for use as inputs to the algorithm's mathematical engine.

\subsubsection{Details of The Mathematical Engine}

The mathematical engine takes in two arrays of sixteen elements each as inputs. Corresponding pairs of array elements are then sent to sixteen sub VI's which perform multiplication and addition operations in parallel. Within each sub VI, three multipliers and one adder can be found. Depending on whether the engine is performing a dot product operation or a filter update operation, the input data will take different paths through the multipliers and adders. Care was taken to minimize the errors associated with loss of fixedpoint precision as much as possible (Figure 80). 


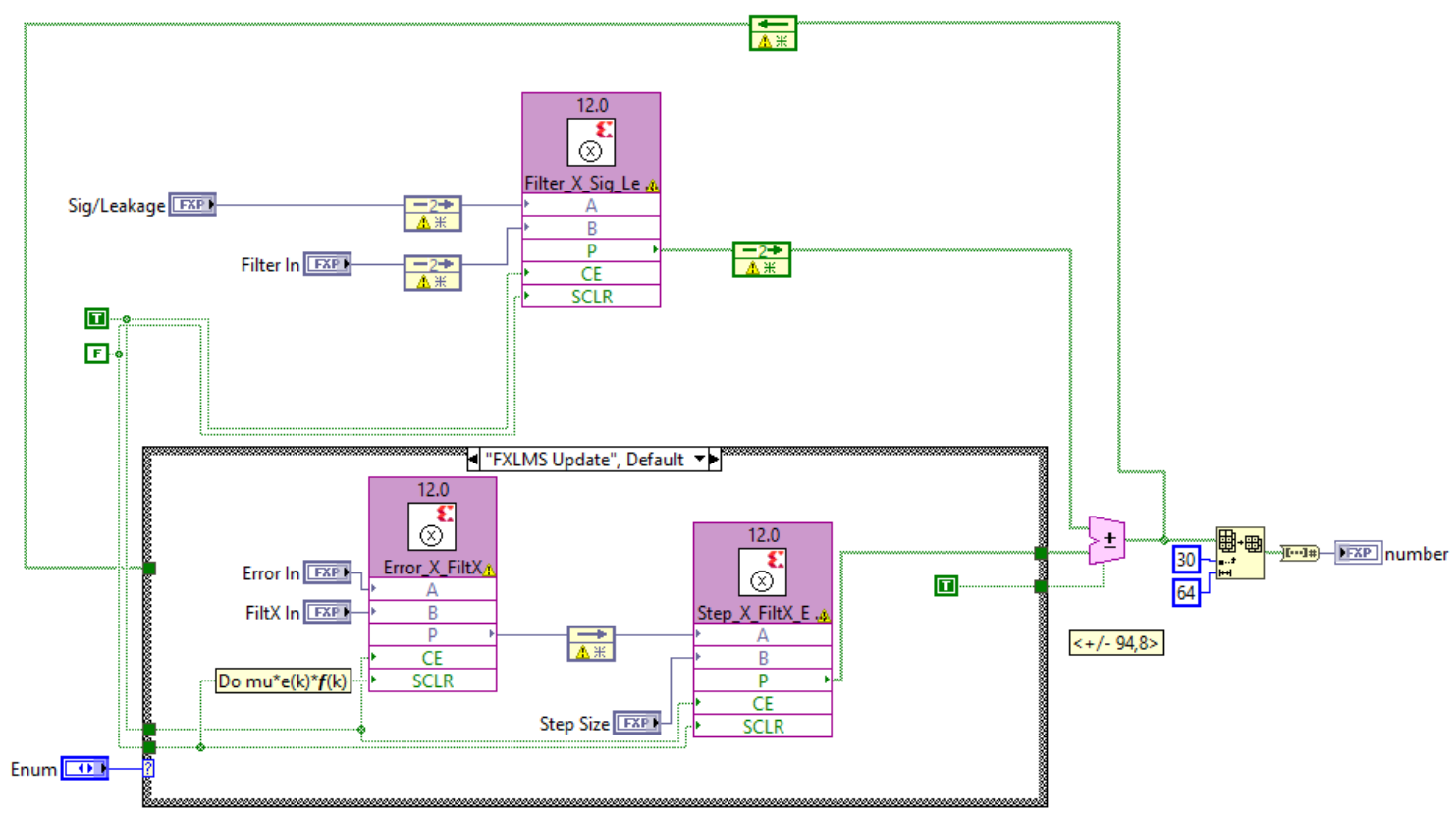

Figure 80: Overview of Mathematical Engine

For the dot product operation, only one multiplier and one adder are used, and data loss is only possible from the adder (Figure 81).

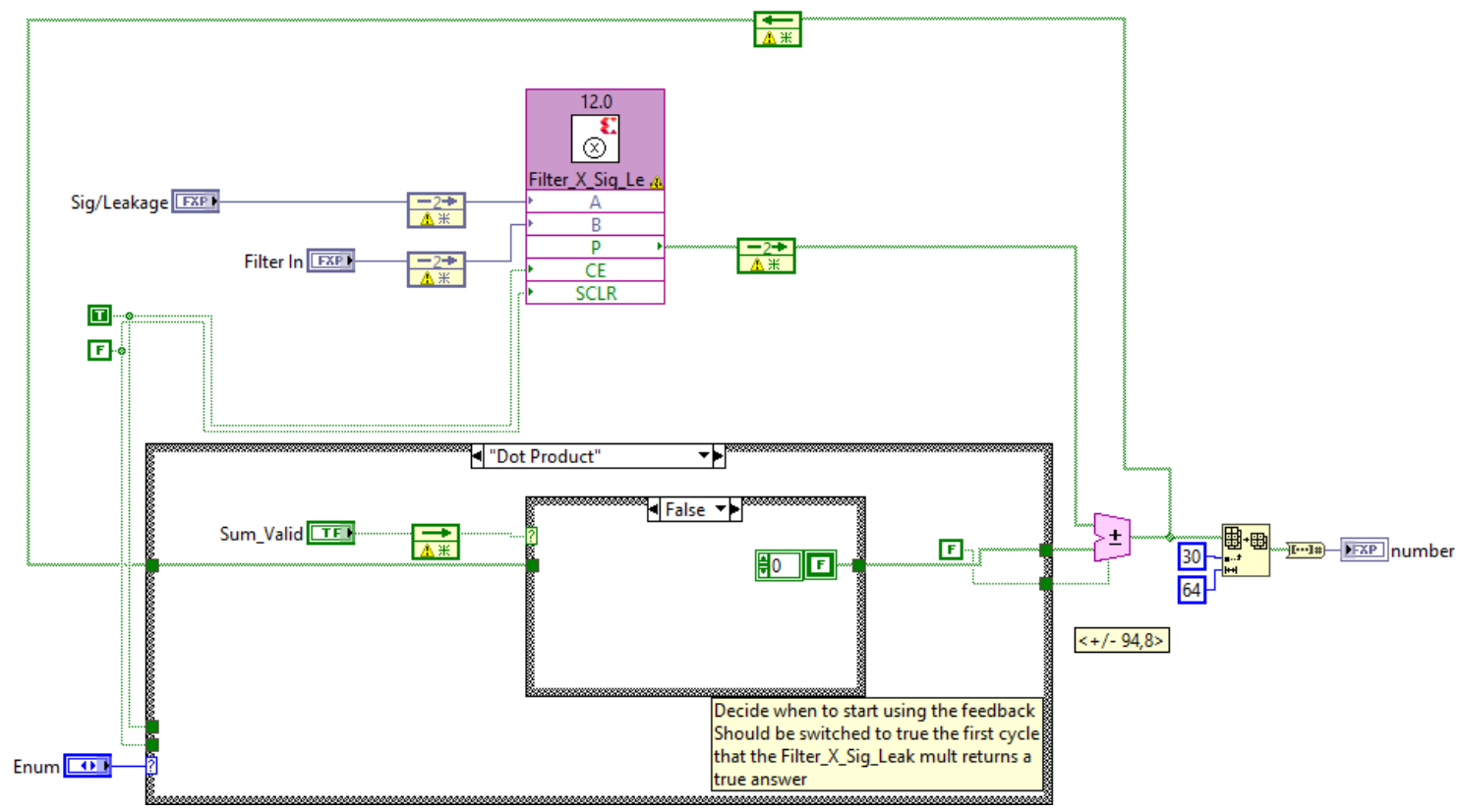

Figure 81: Data Path for Dot Product Operation

As stated earlier the fixed-point precision of signal delay chain and filter tap vectors is $< \pm 47,4>$. Because of this, the multiplier was configured to return a result with type $<$ $\pm 94,8>$, meaning that no bits will be lost. The adder also returns data of the same fixedpoint type meaning that there is the possibility of losing one integer bit at this step. This 
risk is small however, since values are expected to be below one throughout the operation. In order to compute a dot product using this data path the VI is executed in a loop. Each loop will calculate the product of two elements from the signal delay chain and filter taps vectors, and then add this product to a running total using a feedback node. A true/false selector starts this running summation only after the latency of the multiplier stage (14 clock cycles) has been overcome and the results of multiplication are valid. After finishing the dot product operation, the result is truncated to fixed point form $< \pm 64,8>$ so that it can be used more easily throughout the rest of the program.

For the filter update operation all three multipliers and the adder are used (Figure 80) to generate the new FXLMS filter coefficients. The mathematical engine takes five inputs in this case, executing using Eqn. 06 (included below for reference).

$$
\boldsymbol{W}(z)(n+1)=\alpha * \boldsymbol{W}(z)(n)-\mu * e(n) * \boldsymbol{f}(n)
$$

Where $\alpha$ is the leakage coefficient, $\mu$ is the step size, and $e(n)$ is the most recent sample from the error transducer. The first product from Eqn. 06 uses the same multiplier as the dot product operation, returning a result with type $\langle \pm 94,8\rangle$. The second compound product uses two multipliers, the first of which computes the product of $e(k)$ and an element of $\boldsymbol{f}(k)$. Incoming samples from the error transducer are of type $< \pm 24,4>$ since this is the precision of the ADC on the NI9234 I/O card. This multiplier returns a type of $< \pm 59,6>$. While this does allow for some data loss since a full precision result would be type $< \pm 71,8>$, this sacrifice was necessary in order to allow for the step size to have the precision required in the FXLMS algorithm.

The step size, $\mu$, is configured to be type $\langle \pm 35,2\rangle$, meaning that the final multiplier, responsible for computing $\mu * e(n) * \boldsymbol{f}(n)$, will return a result of $\langle \pm 94,8>$. The adder then performs the subtraction from Eqn. 06. It should be noted that unlike the dot product operation, no running total is calculated in the filter update operation. After this final subtraction, results are ready to be written to the FXLMS filter tap vector.

While neither the dot product nor filter update operation cut out fixed-point data loss completely, they still retain a high degree of precision. The dot product operation retains 64 bits of precision while the filter update operation retains 59 bits of precision. When combined, the 16 parallel instances of the sub VIs making up the mathematical engine take up almost all the math resources present on the FPGA. It is difficult to imagine a scenario where the fixed-point precision of the ANC controller could be increased without changing either the hardware or the ANC algorithm.

\subsubsection{Interaction of Vector Element Retrieval and the Mathematical Engine}

Now that the two major building blocks of the algorithm implementation has been laid out, the interaction between these building blocks can be explained. One non-reentrant VI is configured to either compute the dot product between an arbitrary pair of vectors or to update the FXLMS coefficient vector. Several case statements control which memory groups data is retrieved from, determine the correct circular buffer write address to use for address conversion, dictate which operation the mathematical engine will perform, as well 
as other miscellaneous tasks. First, the leakage and step size coefficients are either passed into the $\mathrm{VI}$, or set to zero if the control vector is not being updated. The signal delay chain vector chosen for input then has it's most recent write address read from a register. The single-cycle timed loop (SCTL) then begins to run, and completes 272 iterations. Each iteration, datapoints from two vectors are read, temporally aligned, and operated upon by the mathematical engine. In the case of a filter update operation, the results of this operation are also rounded and written to the FXLMS filter tap vector memory group once per clock cycle. For the dot product operation, the SCTL returns an array of 16 elements, corresponding to the result of 16 dot products between vectors of 256 elements; these elements are then summed in a sequential secondary loop to obtain the final result of the 4096 element dot product (Figure 82).

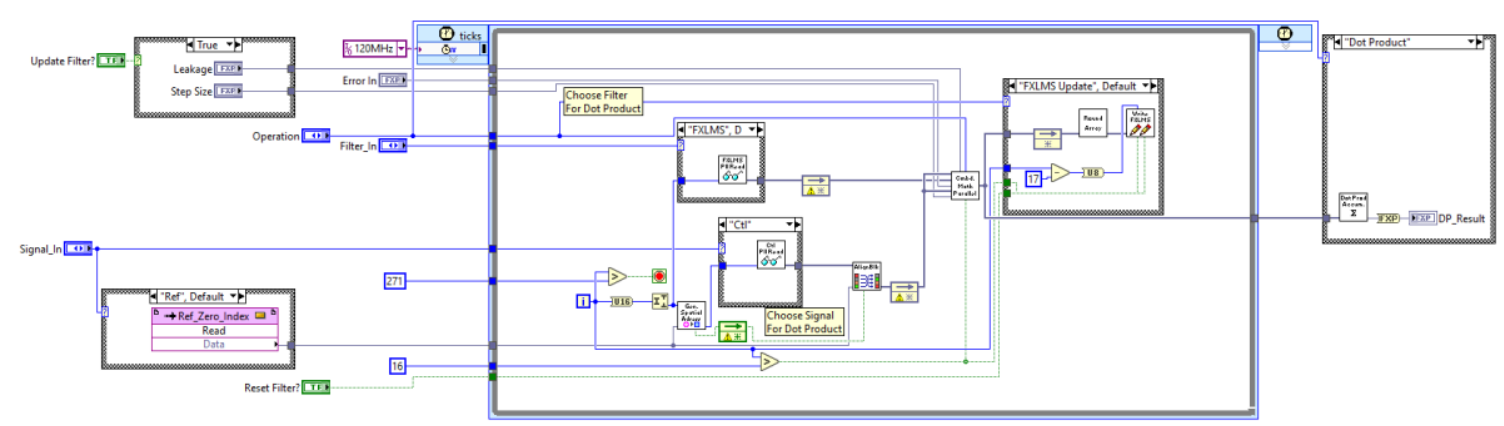

Figure 82: Vector Element Retrieval and Mathematical Engine in a Pipelined Single-Cycle Timed Loop

The SCTL is configured to run at a clock speed of $120 \mathrm{MHz}$, and its throughput is 16 element-wise operations (dot product or filter update) per cycle, with a total pipelining latency of 16 cycles. A dot product operation requires 289 clock ticks to execute while a filter update operation requires 273 clock ticks to execute.

In the FXLMS algorithm this VI performs three dot product operations and one filter update operation (Figure 75), requiring 1156 total clock ticks, between incoming ADC samples. At $120 \mathrm{MHz}$, this equates to an elapsed computation time of $9.63 \times 10^{-6} \mathrm{~s}$. The maximum sampling frequency of the $\mathrm{NI} 9234 \mathrm{I} / \mathrm{O}$ card is $51200 \mathrm{kHz}$, equating to a minimum time between samples of $1.95 \times 10^{-5} \mathrm{~s}$, therefore, the full time-domain FXLMS algorithm is able to respond to an arbitrary input with only one sample of latency.

\subsubsection{PC and Real-Time Components of Controller}

The software and hardware of the cRIO controller exists on three separate levels. The lowest level FPGA software provides the cancellation functionality of the controller and has been explained, so some time will now be dedicated to a shorter description of the remaining two layers. The highest level of the software runs on a PC \& provides a user interface for the controller. This user interface is bridged to the cancellation algorithm using real-time software. The real-time VI mainly serves as a data buffer, but handles some light calculations concerning estimation of the impulse responses for the secondary and feedback paths, as well as the optimal step size. 
On the PC software the user will find a tabbed display with two panes. The first pane has readouts and controls concerning the signal processing parameters and the estimation of the secondary and feedback paths. The number of filter taps to be used in the FXLMS filter can vary between 256 and 4096 in increments of 256. For the secondary and feedback path filters this number can be any integer between 1 and 4096. Sampling frequency can be set to any rate allowed by the NI9234 module. A list of available frequencies is defined by the following function where $n$ is any integer [43]:

$$
f_{s}=\frac{13.1072 \mathrm{MHz} \div 256}{n}
$$

After choosing sampling parameters, the software is started the user must characterize the secondary and feedback paths, which is done by playing white noise through the actuator and measuring the responses at the reference and error transducers. These data streams are run through an LMS algorithm to estimate the impulse responses between the actuator and the two microphones in real time (Figure 83). These calculations are performed on the real-time controller, but live results are visible on the PC. Once the user is satisfied with the estimate of the secondary and feedback path filter taps, cancellation can be started.

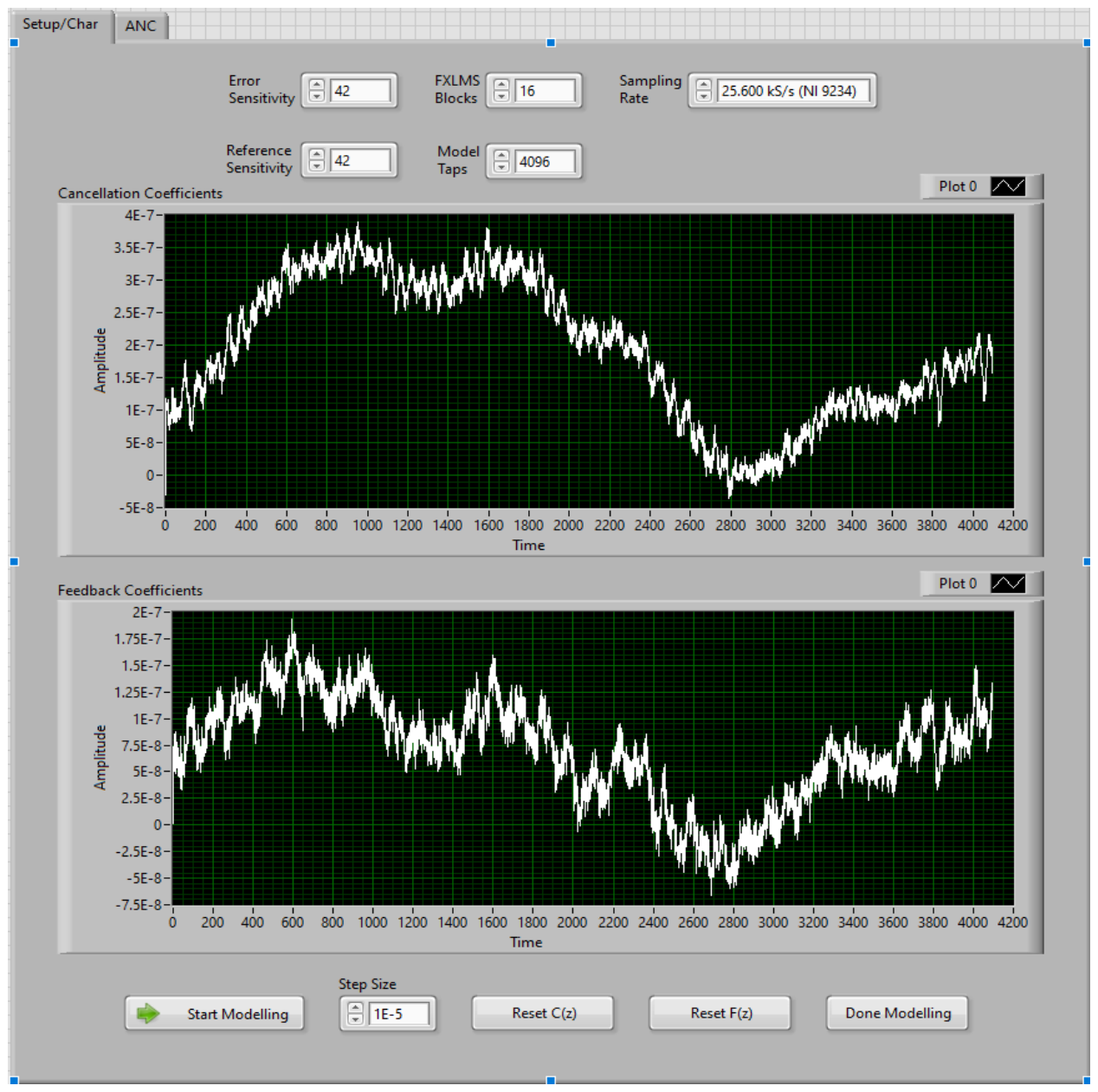

Figure 83: Controller Characterization Page 
On the next pane of the $\mathrm{UI}$ (Figure 84), the user will see time and frequency domain representations of the error, reference, and control signals in real time. A chart which shows the FXLMS filter taps is also visible. Finally, controls are available which allow the user to: turn the control signal on/off, reset the FXLMS filter taps, choose whether or not to update the FXLMS filter, manually tune step size and leakage coefficients, record data, or automatically control the step size of the algorithm.

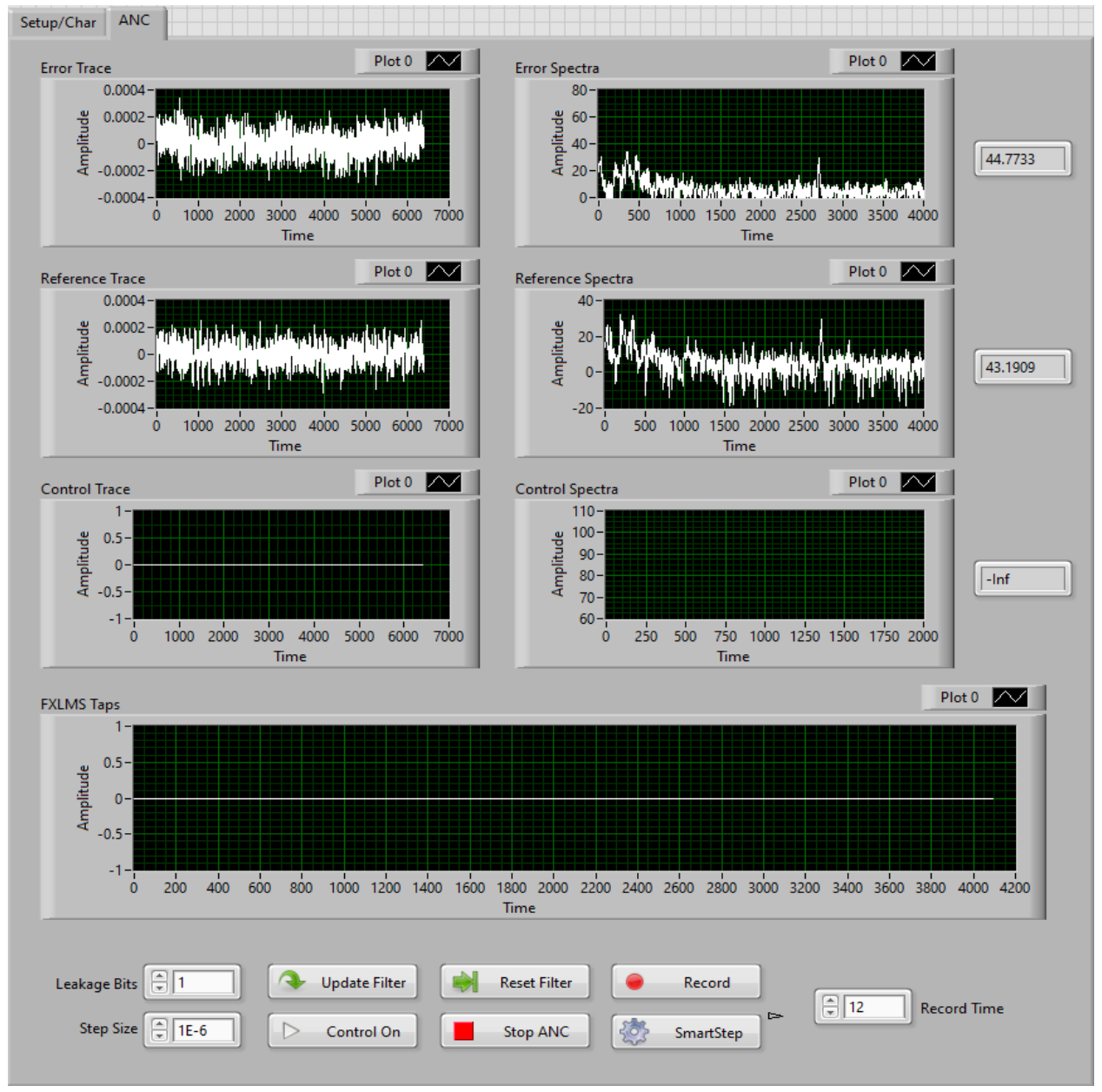

Figure 84: Controller Cancellation Page

In order to get an uninterrupted data stream from the FPGA to the PC for readouts, realtime software is used. Two DMA FIFOs connect the FPGA and the RTC. On one FIFO continuously sends a stream of samples taken by and written from the two $\mathrm{I} / \mathrm{O}$ cards in the system. The next sends the most recent set of FXLMS filter taps every quarter second. The data extracted from these FIFOs is sent to the PC for display but can also be used for calculations concerning the dynamic selection of optimal convergence step size, $\mu$.

This concept of automatically updating FXLMS algorithm step size has been a topic of many papers in the ANC world [44-47]. For this controller, the strategy laid out in this paper was followed [48]. An exhaustive explanation of this control scheme will not be given here as the aforementioned paper does this already. In brief, the controller uses a fuzzy logic 
controller which compares the current error signal to previous error signals and scales the step size relative to the calculated maximum step size.

First, the maximum step size is calculated by inverting the mean square power of the reference signal and dividing by three [9]. The system is allowed to converge towards a solution while the step size remains constant at some arbitrary fraction of this maximum step size. During this convergence, the error signal, and the change between successive samples in the error signal are recorded in two large buffers. The elements in the buffers corresponding to one another in time are then considered as a cloud of points in 2D space, the convex hull of which is used to fit an ellipse. From the matrix representation of this ellipse, a matrix is calculated which will normalize datapoints within the ellipse. The normalization strategy effectively measures to the ratio of the distance between the point and the ellipse center, and the distance between the ellipse center and the edge of the ellipse along a line which passes through the point. As the controller continues to operate, a buffer of these normalized values begins to fill, and the change between successive values is calculated. Both the normalized error values and the change between samples are used as inputs to the fuzzy logic system. The fuzzy controller outputs a value between zero and one which is then multiplied by the most recent estimate of the highest allowable step size for the system. 


\section{Testing Results and Interpretation: Key Findings}

This section of the report will provide fuller detail for the key decision points and findings of the case study, and is complementary to the research path overview portion of the introduction. Go back to that section to fill in any chronological or narrative gaps needed to connect the subsections below. Refer to the chronological account of testing for more details concerning experimental procedures, test setups, and data that was gathered.

\subsection{A Fundamental Shift in Cancellation Strategy}

While a large amount of testing and thermophone development was done in the months leading up to the first ANC demo for the project sponsor's engineering team, it was this demo that stood out as the first truly memorable and significant event of the case study. Not to make light of prior work, it should be noted that important progress was still made before the demo (comments on thermophone development will be presented later). Pursuits during this time period felt almost disconnected, however, and it was not until the demo was developed that the individual pieces really felt like a cohesive system. This can be seen by analyzing the results of this test suite from two different perspectives.

In Figure 21, reduction of two fan-order tones at the error microphone location via ANC is shown. The GLSV controller and RC03 prototype successfully attenuated the $11^{\text {th }}$ order tone and the $13^{\text {th }}$ order tone in the blower fan noise spectra by $8 \mathrm{~dB}$ and $5 \mathrm{~dB}$, respectively. In both cases this was enough for complete elimination of the tone into the broadband floor. The thermophone and controller both functioned perfectly and actually achieved good results if they are considered only as components.

Once perspective shifts and they are viewed as a noise reduction system however, the quality of the results also shifts. Consider the overall sound levels recorded in the three trials: $67.6,69.7, \& 65.9 \mathrm{dBA}$ for the nominal, $11^{\text {th }}$ order cancellation, and $13^{\text {th }}$ order cancellation cases, respectively. After accounting for a third tone that was intermittent across the trials, it is clear that overall levels were not changed in any way that is significant to human hearing. When the components are considered together as a system, the results are poor.

Determining whether this subjective shift ultimately resulted from a change in perspective used to view the components, or from a change in the definition of system success, can be a bit muddy. One thing that that is clear as a result of this, and the testing, however, is that lessons can be learned in both cases. The definition of a cohesive system from the individual components being developed/tested guides the strategy for the remainder of the case study. It was also clear that system success criteria needed to be updated, moving away from a focus on tonal cancellation to a focus on broadband cancellation as the method for achieving overall noise reduction. 


\subsection{First Analysis of Noises within the HVAC System}

The second "aha" moment throughout this case study shares similar themes with the first and provides context for evaluating components within the scope of the system, rather than individually. Specifically, it has to do with the many attempts throughout the project to set targets for thermophone noise output levels.

Preliminary experiments did this using a guided guess and check method. First, the overall level of the blower fan noise in the partition-mount configuration was taken in an anechoic chamber. A lumped parameter model found from CNT literature [11] was then used to predict the amount of CNT required to produce an identical overall SPL. Thermophones were designed around this, then installed into the blower fan. Their performance was verified by playing recreated blower fan noise through the thermophones ( $\mathrm{RC} 01$ iterations) and comparing the resulting spectra to that of the original fan noise. At the time, it was believed that the thermophones met the requirements of the ANC system since they produced a nearly identical overall level to that of the fan (Figure 10).

Unfortunately, this approach suffers from the same problems that were laid out concerning the GLSV controller ANC demo. While level matching the thermophones to the fan in this way is technically sound from an ANC design perspective, it is irrelevant due to the configuration of the blower fan in the experiment not matching that of the actual system. This was not realized at the time since these were among the first experiments performed in this case study.

This became obvious soon after, however, when the same level matching procedure was done using RC03 in the full HVAC configuration. The RC03 thermophone has nearly identical acoustic performance to RC01, and so it was expected that the actuator would match the level of the fan noise in this experiment. It fell well short of this metric instead, producing only $50 \mathrm{dBA}$ at the free-field measurement location compared to the $67 \mathrm{dBA}$ of the blower fan noise (Figure 17).

While exploring this discrepancy seemed important, other threads of the project demanded greater attention at this point in time, so the investigation was put on hold until the integration of the GLSV controller within the HVAC system was complete. The window of time after this, during which a new ANC controller was being purchased, saw the investigation resume.

This investigation was built around validating / invalidating a theory to explain the discrepancy across level-matching techniques. Summarizing the context for this theory: the overall SPL produced by the blower fan at a set distance from either the full HVAC setup or the partition-mount setup was roughly the same, whereas this was not the case for an identical thermophone producing noise in either configuration. Hence, it was originally theorized that the explanation could be found in the propagation of sound through the two systems. Specifically, the noise from a thermophone was thought to attenuate more rapidly than noise caused by the blower fan as both sources propagated through the full HVAC setup. 
Physically this is impossible. While this was not shown directly by the measurements taken during this experiment, dismissing this original theory was still one of the conclusions of this experimental suite. Luckily however, a different explanation was also among the conclusions.

So, identical noise sources ${ }^{8}$ inside the blower fan, whether they be the fan itself or the thermophone prototypes, will all be attenuated in the same way as they travel through the HVAC system. Exclusively in the case of the blower fan noise, however, measurements taken also contain sound contributed by an additional source. The turbulent boundary layer of fast-moving airflow interacting with the interior surfaces of the HVAC acts as a noise source distributed throughout the system [49-51]. Think of this like a mesh surface coating the inside of the HVAC that emits air rush noise continuously across its entirety. This will be called the "continuum of point sources." This separation of noise sources within the HVAC will become very significant to the future of the project.

This insight is already valuable, but more can potentially be gained by looking at results from this experiment. Before we continue it should be noted, however, that the following analysis has not been rigorously cross-examined with acoustic theory. It is a hunch that could explain system behavior which seems to align with literature about HVAC noise and has not been proven yet. It is up to the reader to choose whether to consider the remaining paragraphs in this section (barring the final, summary paragraph) when making decisions for the design of ANC systems.

Two paragraphs prior, the blower fan noise originating from inside the fan, and the two prototype thermophones were considered as "identical" noise sources. Consider Figure 35. The "FRFs" between the two microphones are nearly the same for the three sources in the partition-mount configuration. Don't forget the limitations placed on these calculations however, and that they are not true system input / system output comparisons. Despite this, these results do not eliminate the possibility that the acoustic fields independently generated by the three sources could be identical outside the blower fan exhaust in the far field. This is most likely to be true at frequencies less than the cut on frequency of the ducting, where plane waves form [52-54]; most sources indicate this to be unlikely but possible at higher frequencies. Where this is the case however, the thermophones should be well equipped to achieve global attenuation on the blower fan noise in this configuration.

Why then, does the full HVAC system counterpart to Figure 35, Figure 36, indicate that the relationship between "FRFs" is different for the two thermophone sources? Surely if the acoustic fields were "identical" this would not be the case. Well, it depends on where observation of the acoustic fields takes place. At some point in either setup the noises propagating from the sources transition from being "in the fan" or "in the HVAC" to being "in the anechoic chamber." If the sensitivity relating the acoustic field shapes before and after this transition were different across the two cases, this could explain why Figure 35 indicates the three sources to be "identical" and Figure 36 does not.

\footnotetext{
${ }^{8}$ Continue reading for a potential explanation as to why these sources might be considered
} "identical" 
Specifically, it is suspected that when the acoustic field transitions from being contained within a duct to the free field, the aforementioned sensitivity is very low. Effectively this states that a complicated field within a duct will result in a very simple field in the free field. In the case of the partition-mount trials, both microphones are in the free field, on the same side of this transition. In the HVAC setup, one microphone is in the complicated field contained by the ducting, and the other is in the simplistic free field. The two microphones being located on either side of this transition causes the discrepancies across sources seen in Figure 36. If this analysis is true, consider the ramifications of this that affect an ANC system designed for the full HVAC setup. Inside the ducting it is likely that cancellation will not occur since the fields will not be identical. Outside of the ducting, past the transition to the free field, the opposite should be true.

Even so, prior analysis of this section shows that successful cancellation of only blower fan noise originating in the fan itself will not change overall levels outside the full HVAC setup significantly since the spectrum at this location is not dominated by this noise source, but instead by the "continuum of sources" caused by the turbulent boundary layer (Figure 17). Building on this, none of the analysis in this section considered the secondary path that blower fan noise originating in the blower will take to the vehicle's occupant. In order to achieve significant reduction, both noise sources in the HVAC system, and all paths that these sources can travel through to the receiver, must be considered.

\subsection{Why the Tiger WIFI-S both Works and Doesn't Work}

It was stated earlier that the results of testing with the Tiger WIFI-S were mixed. The controller has been demonstrated to be successful in cancelling tonal and broadband noise in both free-field and ducted setups using a variety of disturbance sources (Figure $47 \&$ Figure 53). Changing the configuration of these setups can drastically impact results, however. The most noteworthy example of this is the transition to a blower fan disturbance noise source in the ducted setup, a change which has made significant cancellation unattainable to date. Because of this contrast it is obvious that there is some set of limitations placed on the ANC setup, which must be met in order for cancellation to be achieved.

The usage of "limitations" in the prior paragraph is intentionally vague. For most of the case study, an exact definition of these limitations which could be useful for ANC system design had not been laid out. After hitting a wall with testing the Tiger WIFI-S, an effort to do this began. Doing so would not only explain why the Tiger WIFI-S works in some scenarios and not others, but could also bring to light as a set of constraints useful for future designs of an HVAC ANC system.

Based on background research into ANC and observations from past tests, a minimum viable set of system characteristics linked to these limitations was theorized. The set was comprised of microphone coherence, and system causality [30] [55]. Put simply, the spirit of this theory is that a system with good microphone coherence and system causality should always yield cancellation results, whereas a system failing to meet these criteria should not. This was put to the test in the experiments described here. 
Figure 59 shows the relationship between reference/error microphone spacing on cancellation performance across test trials. While causality-centered testing was initially thought to be mostly a formality (it is well documented in the literature that system causality is required for broadband cancellation), an interesting observation was made from the results. Since causality describes the ability of an ANC system to meet specific timing thresholds, it is a binary criterion. This being the case, it follows that in an experiment where causality is dictated by the independent variable, results for the dependent variable should also be binary. Either cancellation would occur, or it would not; the magnitude of cancellation should not be affected. Expected results (with an arbitrary microphone spacing, $13 \mathrm{~cm}$, being the cutoff for system causality) would look like Figure 85:

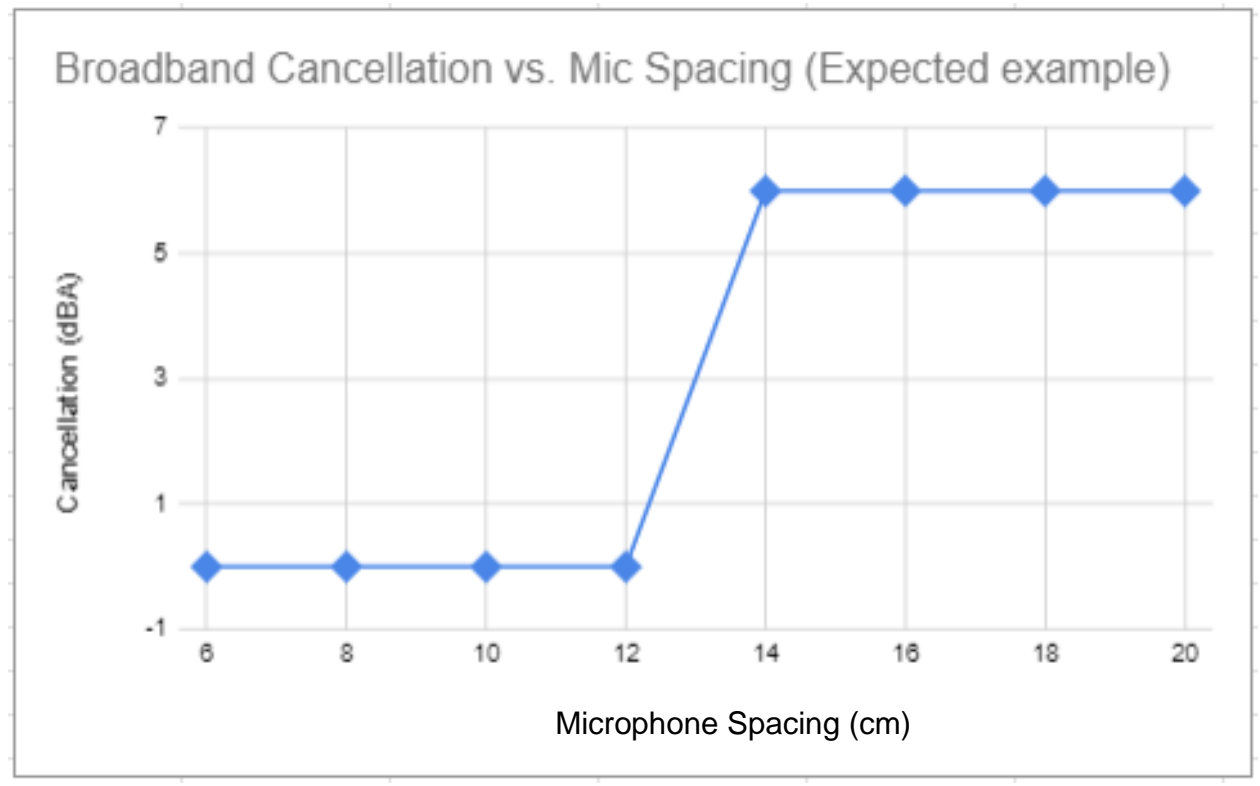

Figure 85: Expected Results from a Causality-Centered Cancellation Experiment

Seeing the deviation from expected results actually reveals another limitation to be added to the theorized minimum viable set described earlier. Acoustic feedback from the actuator to the reference microphone would cause the behavior seen in Figure 59 [56]. Additionally, it explains why cancellation performance is degraded in a ducted setup when the transition is made from the disturbance signal to a microphone as the origin of the reference signal.

So, with the length of a minimum viable set of "limitations" now expanded from two to three, more consideration will be given to the results from this testing suite. Coherence between microphones was also found to influence cancellation levels in the free-field (Figure 61).

For all of the free-field cancellation trials, the coherence and cancellation levels within an arbitrary bandwidth were calculated. These points were used to fit a regression that defines the relationship between coherence and cancellation for that band (Figure 86). For now, an example band is shown, but one of greater significance will be touched on later. Even from the example band, however, it is clear that there is a relationship between coherence and cancellation level achievable in the free field. 


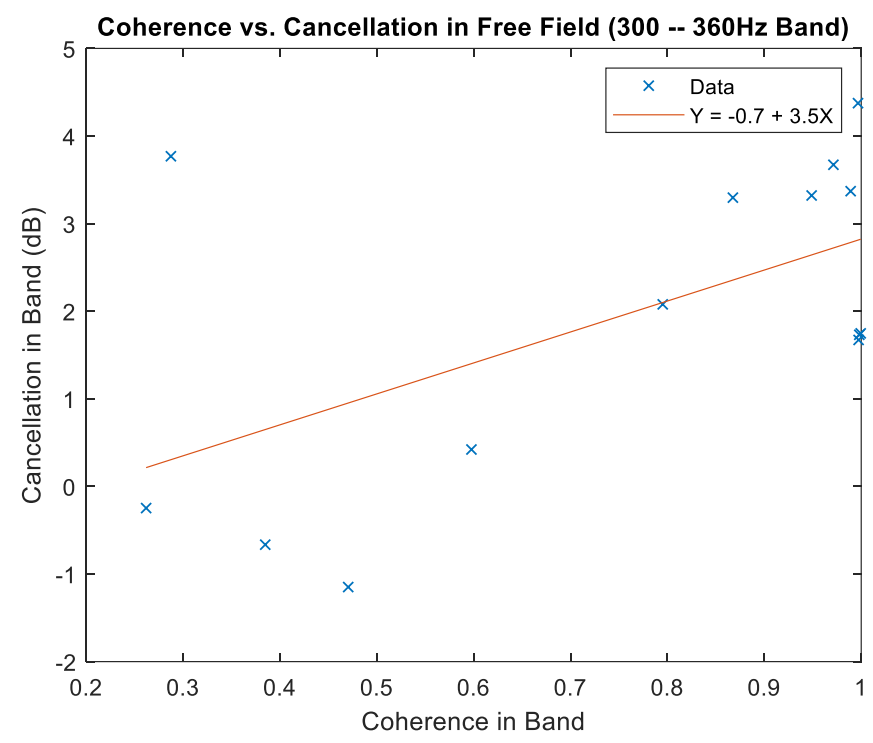

Figure 86: Example of The Relationship Between Coherence and Free-Field Cancellation Levels for an Arbitrary Band

In a ducted system it is strongly suspected that this is the case, but experimentation could not validate this. The level of cancellation achieved with near perfect coherence was very low in a ducted setup (Figure 63). As coherence was decreased so too was the cancellation, but when the results are analyzed in a similar way to Figure 86 a good regression fit is not possible (Figure 87). All the datapoints are too close together, and for most trials, levels of the pre and post ANC spectra are too close to ensure that any observed "cancellation" is not just normal measurement variation (Figure 63).

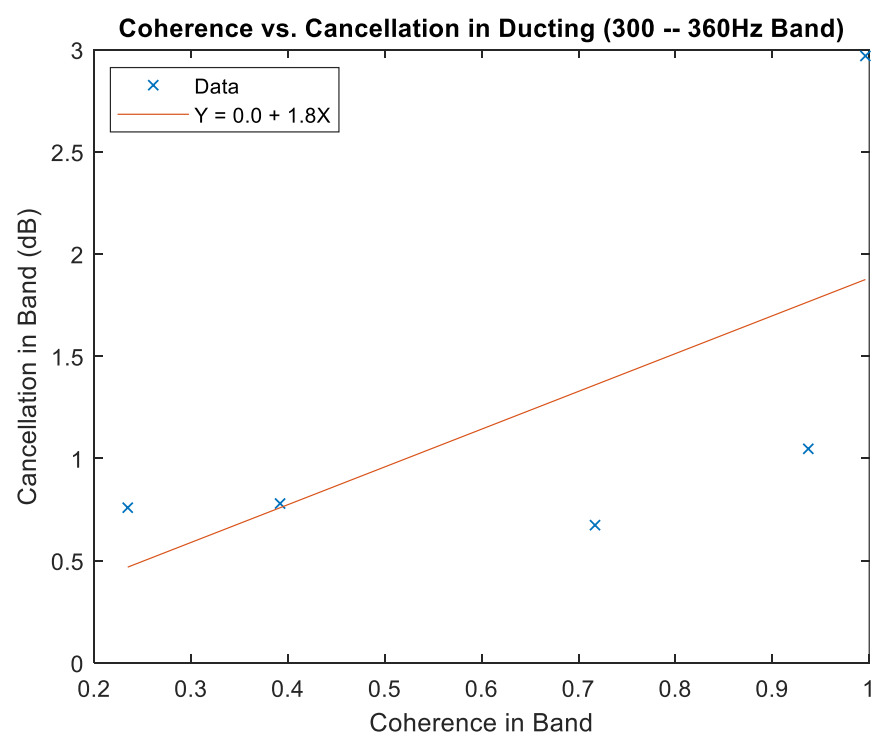

Figure 87: Example of The Relationship Between Coherence and in-Ducting Cancellation Levels for an Arbitrary Band 
In summation of this section, evidence has been shown that three theorized system characteristics, microphone coherence, system causality, and acoustic feedback, all impose limitations on the system that must be met for cancellation to occur. It is important to note here that the experiments performed do not offer rigorous proof of this theory. Nor do the experiments preclude the existence of other, still undiscovered, "limitations" that might be added to the minimum viable set in the future. However, this theory does explain all of the behaviors noted during testing with the Tiger WIFI-S, specifically, why it performs well in one setup and poorly in another.

\subsection{Can What Prevents the Tiger WIFI-S From Cancelling be Solved?}

After all of that work testing and analyzing the Tiger WIFI-S, a theory outlining a minimum viable set of "limitations" that dictate controller performance in an ANC system has been proposed. In the interest of continued analysis, assume that the theory is valid for now. Credence is also lent to this assumption by a variety of sources throughout this report [30, 55-58]. If that is the case, what does that mean for the future development of this ANC system? Currently a ducted, high-airflow environment falls outside the realm of these limitations, but can this be remedied?

To see if such an environment can be molded to fit within the proposed limitations, each limitation is approached independently. First to be considered is causality. This one is easy, as ANC setups using the Tiger WIFI-S have already demonstrated causality at microphone spacings as short as $12 \mathrm{~cm}$ (Figure 59). This spacing is already quite low, and two microphones with this spacing fit comfortably within the geometry of the HVAC ducting at many locations. This minimum spacing also stands to be decreased with the development of an FPGA-based ANC controller [59]. In short, causality does not seem to pose a serious problem for the HVAC ANC system.

Coherence, on the other hand, does. Looking back at the relationship between coherence and cancellation can reveal more about this. Initially this relationship was developed for an arbitrary band as an example of the calculation technique (Figure 86). What band should this analysis be focused on, however? The spectra for blower fan noise in the partition-mount configuration ${ }^{9}$ can be used to choose bands of particular interest for targeting with cancellation (Figure 88). Choice is subjective based off what regions in the spectra are highest, as reduction here should impact overall level change the most.

\footnotetext{
${ }^{9}$ Is this the same mistake as here? At this point in the case study all efforts were being focused on getting cancellation within the partition-mount PVC duct demo configuration, so no, if results are interpreted within the context of this system.
} 


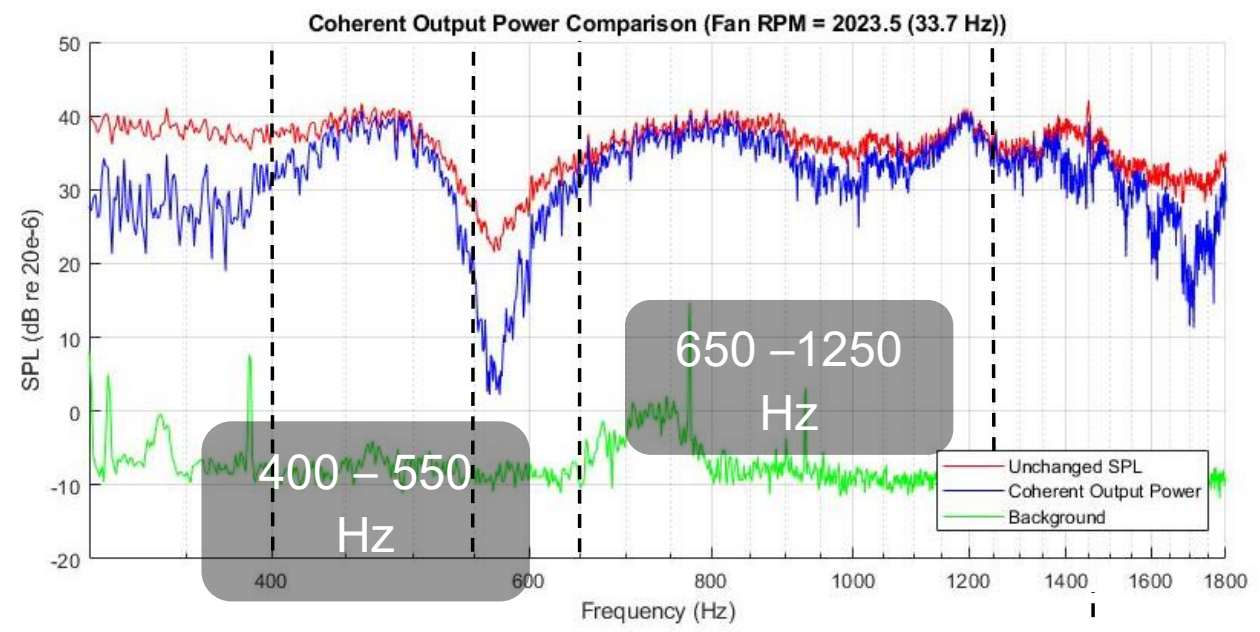

Figure 88: Selection of Bands to be Targeted by Cancellation for Maximum Impact on the Overall SPL

Within these bands, the same analysis as that done to produce Figure 88 was performed, once again using data from the free-field trials. In both cases a plausible relationship between coherence and cancellation was found (Figure 89).
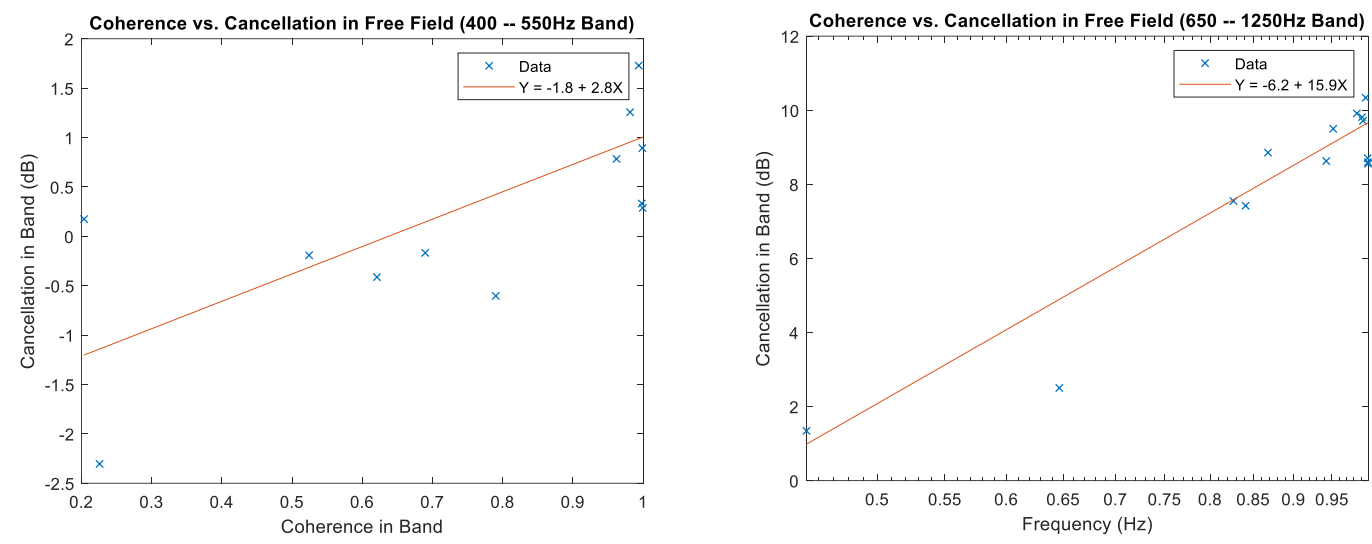

Figure 89: Relationship between Coherence and Free-Field Cancellation Levels for two Frequency Bands

Resulting from the two regressions performed are the following equations, that describe the coherence/cancellation relationships for the $400-550 \mathrm{~Hz}$ band and $650-1250 \mathrm{~Hz}$ band, respectively.

$$
\begin{aligned}
& \text { Cancellation }=-1.8+2.8 * \text { Coherence } \\
& \text { Cancellation }=-6.2+15.9 * \text { Coherence }
\end{aligned}
$$

Eqn. 8

Eqn. 9

Where cancellation is the level of attenuation achieved in that band $(\mathrm{dB})$. With these equations the pieces of the coherence puzzle are almost ready to be put together, but first one more figure must be considered. At the project sponsor's facility, testing was done on a technique used to improve microphone coherence in high-airflow ducting. These equations will be applied to the coherence of blower fan noise across the microphones shown in Figure 55 (Figure 90). 


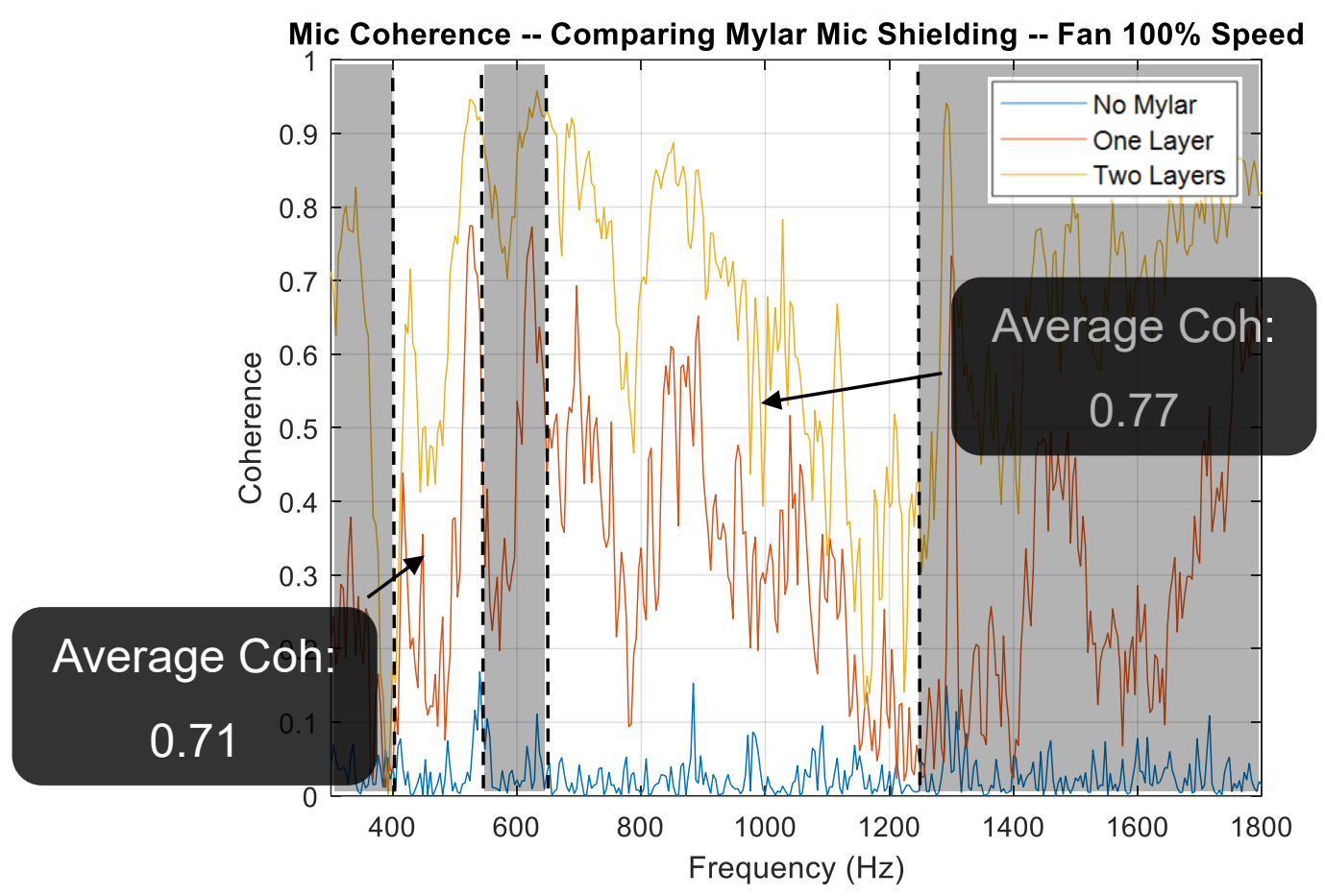

Figure 90: Best-Case Coherence of Blower Fan Noise across Two Bands in a Ducted High-Airflow Setup

Using the "Two Layers" curve from Figure 90, average coherences across the $400-550$ $\mathrm{Hz}$ and $650-1250 \mathrm{~Hz}$ bands are 0.71 and 0.77 respectively. Using equations $5 \& 6$, the levels of cancellation attainable for these bands are predicted to be $0.2 \mathrm{~dB}$ and $6.0 \mathrm{~dB}$, again, respectively.

What is the impact of these predictions? Reduction of noise in a band by $0.2 \mathrm{~dB}$ will not alter the overall level of the fan spectra significantly. A $6 \mathrm{~dB}$ reduction over the second, wider, band, is more promising however. Based on the current fan spectra (Figure 88), an overall level change resulting from this will still likely be no more than a few $\mathrm{dB}$, but it is a good start. If microphone coherence can continue to be improved through other means, cancellation results should improve as well [58]. Whether or not this is possible in this is left up to the judgement of the reader as asserting one way or another at this point would be speculation. Keep in mind, however, that the 15-minute exercise of taping two layers of mylar sheet over the microphone's flush mount holes has already made a drastic positive impact on the coherence between microphones in high-airflow. Other researchers have also documented improving ANC performance in a ducted environment with flow, albeit within a low frequency range and with relatively low flow speeds $[60,61]$.

These findings about the coherence might lead one to wonder why it is that in ducted highairflow testing, cancellation to some degree was not seen across the bands of interest discussed above after coherence was improved using mylar. The likely, yet somewhat unsatisfying answer is that acoustic feedback causes this [56, 57]. The exact nature of how feedback affects this system has continued to be unclear and a relationship to cancellation like that found for coherence has yet to be uncovered. 
It was because of this obscurity that the decision was made to focus on eliminating the feedback neutralization problem before coherence was tackled. Consider two different ducted configurations in which cancellation is attempted. The first configuration uses a loudspeaker as the disturbance and has no airflow, the second uses the blower fan for the disturbance and has airflow. Coherence only presents a problem in one setup, whereas feedback presents a problem in both, assuming a reference microphone is used in either case. Because of this, no incremental gains in performance would be made by improving coherence without first fixing feedback neutralization problems.

In summation of this section, some predictions concerning potential for ANC system performance increases were made. Causality does not seem to present a problem for the system. Coherence does, however. Evidence suggests that improving coherence levels will lead to increases in cancellation performance ${ }^{10}[60,61]$; predictions of future improvements to coherence are speculative, but optimistic [58]. Finally, the exact effects of acoustic feedback on cancellation levels have yet to be discovered, but it seems that this issue must be solved before system performance stands to improve by any other means.

\subsection{A Proposed Model for Use in Ducted ANC Systems Design \& What it Can Tell Us}

So far two major threads have been discussed in this results section: what is the noise coming from the HVAC really like, and why are cancellation results low for the HVAC system? After introducing a simple model to be used for designing ANC systems going forward, these threads will be merged in a short description of an optimal ducted ANC system.

One disclaimer before this is started. This model has not been run through the rigors of cross-analysis with the acoustic wave equation and is not intended to be used quantitatively. Instead, it is a practical, qualitative model based off testing observations that is intended to guide the direction of ongoing research at a high level. There is a possibility that this model is incorrect at some granular acoustic level, but so far it aligns with what has been observed from testing the system as well as with simple duct acoustics. With that out of the way, here is a description of the model.

In analysis on noise within the HVAC laid out earlier, an argument was made that the "blower fan noise" heard by an observer outside the HVAC is actually a combination of contributions from two different noise sources. The first source ${ }^{11}$ is noise from inside the fan itself. The second is the "continuum of point sources" which represents air rush noise in the HVAC. Their relative contributions to the overall noise will be ignored for now.

\footnotetext{
${ }^{10}$ Evaluate this claim within the context that this section only considers a one-duct system, and that in the real HVAC system, additional noise sources and paths to the receiver are present. ${ }^{11}$ Really this is many sources, but it will be considered as one for now. Read the last few paragraphs of this section for more clarity on this.
} 
For the time being, think about their propagation within a duct. Within ducting, a sound wave generated by a point source will transform into a plane wave only after reaching a specific distance from the source (Figure 91) [62].

\section{Point Source}

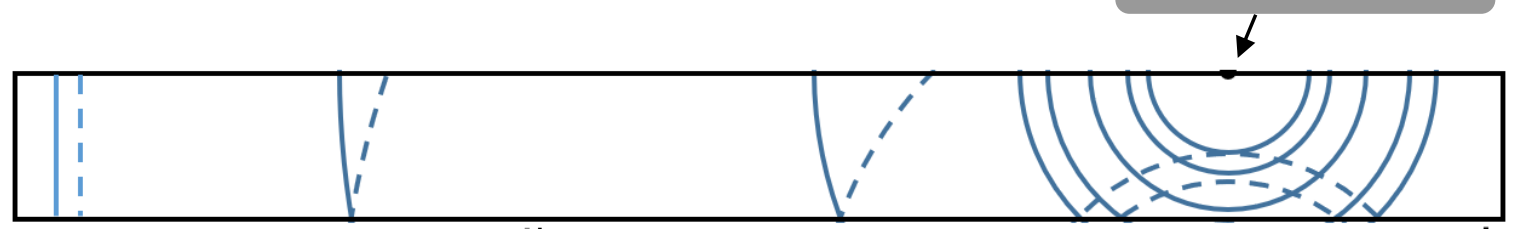

Figure 91: Direct and Reflected Waves Propagating Away from a Point Source in a Duct

With a little help from your imagination, Figure 91 shows a side section view of a duct with an acoustic point source stuck to its inside wall. Solid lines represent direct waves, and dashed lines represent waves that are reflected from the wall opposite the point source. Near the point source, the two wave fields have very complicated interactions, especially if a third dimension is considered. As one moves to the left and away from the point source however, the two wave fronts become more and more parallel. Eventually the result is a plane wave.

So, for any point source, two zones relative to the position of the point source can be defined. One zone contains plane waves, and the other zone does not (Figure 92).

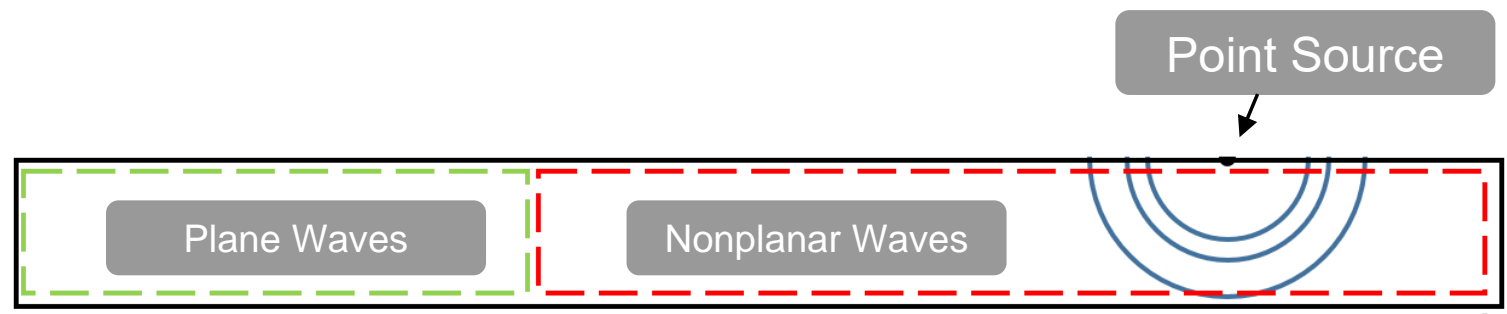

Figure 92: Separation of Ducting Containing a Point Source into Two Zones Containing Different Wave Types

According to conventional ANC theory, only plane waves are controllable within ducting if a single channel ANC system is used [63]. Thus, if both microphones and the actuator are placed within the green "plane wave zone," noise from this point source should be cancellable using a single-channel controller. Placing these components in the red "nonplanar wave zone," however, will result in cancellation being impossible. For this incredibly simple example duct, this presents no problem.

When a continuum of point sources is considered things start to get more complicated. This is important since air rush noise is represented as such in this model. With infinitely many point sources now occupying the walls of the ducting, it no longer makes sense to define two zones relative to each point source. Instead, zones are defined relative to the exit of the ducting. The first zone will contain point sources which are far enough away such that sound originating from them will have developed into a plane wave by reaching the duct exit. The second zone contains point sources fitting the opposite case. Point sources within the first zone should be cancellable while those in the second should not 
(Figure 93). It is assumed for simplicity that the "blower fan noise" falls into the first zone since the blower fan is opposite the duct exit.

\section{Point Sources}

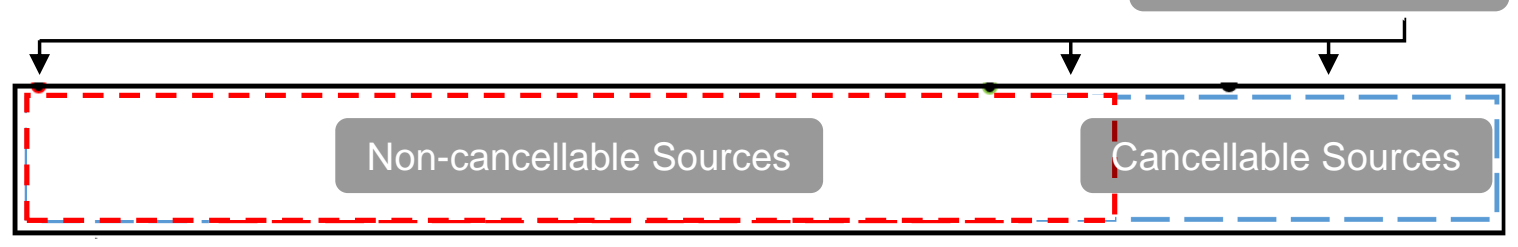

Figure 93: Definition of Zones Relative to the Duct Exit Which Contain Either Cancellable or NonCancellable Point Sources

Now consider what would happen if the reference for defining these zones was taken to be the ANC transducer group (actuator \& error/reference transducers) instead of the duct exit. If the transducer group were moved within the ducting, the zones would also move accordingly. Specifically, the red "non-cancellable" zone always extends the same distance from the transducer group in both directions. In order to maximize the size of the "cancellable" zone within the ducting then, the ideal location for the actuator group is in fact at the duct exit.

This model suggests that the ANC transducer group should be placed as close as possible to the duct exit, but this is not the only conclusion that can be drawn from it. The same zones from Figure 93 also show that minimizing the spacing between the reference and error transducers should be a design goal. Since any given sound must be a plane wave at both the error and reference transducer, the reference transducer specifically (not the whole transducer group, as before) becomes the origin of the two zones. Now the reference transducer must be as close as possible to the duct exit, and therefore the error microphone as well, if the size of the cancellation zone is to be maximized.

To summarize, the model for noise sources inside a ducted system subject to a blower fan disturbance suggests minimizing the spacing across the ANC transducer group, and that the transducer group be placed as close to the duct exit as possible. Doing so should maximize the subset of noise sources within the duct which are cancellable. In order to most effectively execute cancellation on this subset, the transducers must have good coherence, acoustic feedback must be neutralized, and system causality must be maintained.

All evidence suggests that an ANC system which follows these guidelines will provide the best possible cancellation performance for the single duct case considered here. Unfortunately, a concrete prediction as to overall reduction levels such a system could attain would be speculation given the information collected in this case study. 


\subsection{Realization of The Proposed Optimal Ducted ANC System}

Based on the model proposed in the previous subsection, a new ducted ANC test setup was built. Since the general physical configuration of the proposed model only requires that the transducers be closely spaced and near the duct outlet, the form factor of the realized optimal system has not changed much relative to previous in-duct cancellation setups. It still uses the 4" PVC which replicates a section of HVAC ducting and houses the transducer group. The transducer group consists of two $1 / 4$ " array microphones which serve as reference and error transducers; two different actuators were used throughout testing. Both the annular aluminum thermophone and the annular loudspeaker array served this purpose (Figure 94).
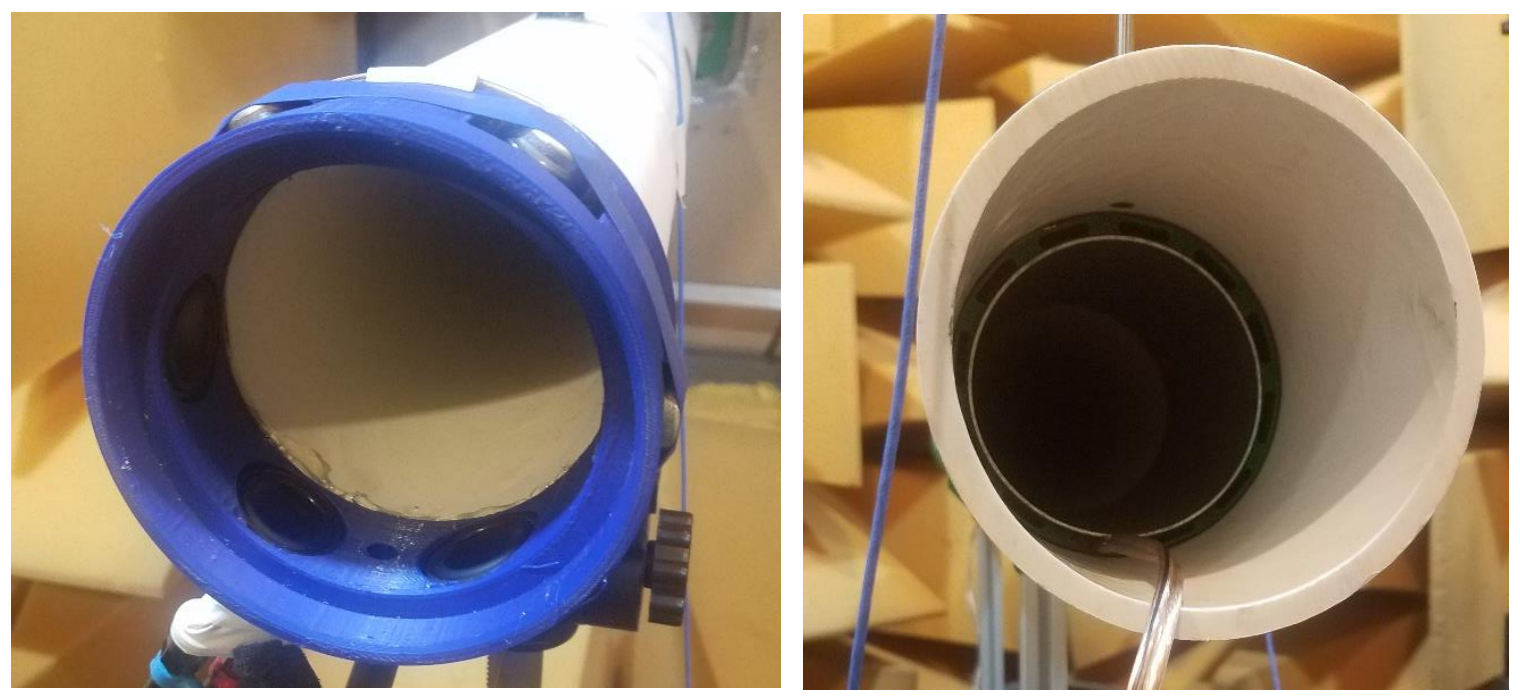

Figure 94: Annular Loudspeaker Array and Annular Aluminum Thermophone Installed in 4" PVC Ducting for Noise Cancellation

One important non-physical constraint placed on the proposed optimal model is that the reference and error transducer signals be highly coherent. As was shown in prior testing, coherence between the microphones can be increased using a special mounting technique. The microphones are flush mounted inside the PVC pipe, albeit with their heads slightly recessed from the inner surface of the pipe. The microphone mount holes were then covered with a thin film of plastic tape to reduce air rush noise at the microphones (Figure 64). The final constraint placed on the proposed optimal model is that is must have some mechanism to attenuate acoustic feedback. In the realized system this is achieved via the use of an ANC controller which removes feedback from the reference signal digitally as part of the noise control algorithm.

Since the purpose of creating an ANC system conforming to the proposed optimal model was to validate the correctness of the model, several rounds of testing were performed using this penultimate setup. Observing Figure 67 - Figure 73, one can see the results of this testing. Initial tonal results look very strong, with the controller being able to eliminate pure tones completely using both CNT and loudspeaker actuators. Broadband results, however, are not as good. 
In the case of the annular aluminum thermophone actuator, the overall level of a broadband disturbance was attenuated significantly, up to $11.6 \mathrm{dBA}$. However, the width of the excitation band for the disturbance is only $100 \mathrm{~Hz}$; after trial and error this was found to be the only "broadband" disturbance which was controllable. So while the overall reduction level is good, the results are still not meaningful if the disturbance excitation range cannot be increased. This already doesn't bode well for the proposed model since in the absence of fan noise, all broadband disturbances within the duct have near perfect coherence. This, plus the addition of an acoustic feedback feature to the controller means that some other factor is limiting the performance of the controller.

To verify that this unknown limitation was not being imposed by the CNT, the actuator was changed to be the annular loudspeaker array at this point. This actuator is much more simple acoustically. Broadband trials were continued, this time with a wider excitation band (Figure 70). It was found from these trials that the change to a simpler actuator was not able to significantly increase the broadband performance of the controller. In fact, the cancellation results from this trial using the new system look quite similar to the results from the older system which uses the Tiger WIFI-S controller (Figure 63). In both cases, only the noise caused by pipe modes was able to be cancelled.

Another unexpected similarity between testing with the proposed optimal system and the Tiger WIFI-S system was the broadband cancellation behavior change when the disturbance signal was substituted for the microphone as the reference signal. In the proposed optimal system the broadband cancellation was also found to be greatly increased should this be the case (Figure 71). While this does not logically disprove the theory that acoustic feedback limited the performance of the Tiger WIFI-S system, it also does not prove it to be the case, and in fact suggests that there is another limitation which is yet to be discovered.

One theory about this comes from [30] and has to do with the autocorrelation matrix of the reference signal. Pp. $405-406$ show that the convergence of the FXLMS algorithm is greatly dependent on the autocorrelation matrix of the reference signal. Specifically, the speed of convergence is inversely proportional to the ratio between the magnitude of the largest and smallest eigenvalues of this matrix. This is due to the partial gradients of the error surface along its principal axes being proportional to the eigenvalues themselves. Essentially this means that the algorithm will converge faster in some "directions" along the error surface than others. As is also stated in the text, for signals containing large tonal components this eigenvalue magnitude ratio is typically very high. This, coupled with the data losses associated with fixed point computations, could mean that along the principal axes corresponding to very small autocorrelation matrix eigenvalues, the matrix cannot converge due to truncation error; the amount of distance covered in that direction could be less than the minimum precision of the system.

Considering this potential explanation alongside the observation that broadband excitation with a loudspeaker through the PVC pipe is prone to producing tonal pipe modes, cancellation of a different source was attempted. Fan noise was chosen as this final source since it does not excite pipe modes and is the disturbance which this whole case study seeks to cancel in the first place. Unfortunately no cancellation was achieved, despite several regions along the spectra having coherence at or above 0.9 (Figure 72). 
Overall, this test suite showed decisively that the model used to infer the proposed optimal ANC system does not characterize the HVAC system in a way that is useful for ANC system design. The test suite also showed that the cRIO FPGA controller performs at a level at least as high as a commercial component, and that the dynamic step size feature is working as intended. Observing that the addition of acoustic feedback neutralization did not remove the performance discrepancies across systems using different reference signal acquisition methods (microphone vs. tapped disturbance line) was another unexpected and interesting result of this test suite. More investigation into explaining this discrepancy would make an excellent continuation of this work.

\subsection{Comments on the Viability of the Thermophone in this Case Study}

It may seem odd to the reader that despite the huge role played by thermophones in this case study, their analysis is the last to be mentioned. This decision was made so that the full context of all challenges faced in other parts of the ANC system could be introduced first. Knowing this will help better define the requirements of the actuator as a system component. Only then can the viability of the thermophone be judged.

\subsubsection{Acoustics - Replication of the Acoustic Field From Two Sources}

Consideration will be given to the acoustic viability of the thermophones first. In order to do this, a summarization of the understanding of the full HVAC system now that all the results and analysis surrounding this have been laid out is forthcoming. The vehicle's occupant hears HVAC noise. This HVAC noise is a combination of two different sources within the HVAC system: the blower fan noise (originating in the blower fan), and the air rush noise which is generated continuously throughout the HVAC. Additionally, the blower fan noise takes two paths to get to the occupant: through the evaporator/mixing chamber and out the ducts, and through the fan's intake and out from underneath the passengerside dashboard. Overall, there are three source/path combinations, all of which must be addressed to get maximum cancellation performance out of the system. Most of the work done in this case study focuses on the two sources and their paths through the HVAC, not out the fan intake. For one of these source/path pairs, the thermophones still present themselves to be potentially viable acoustically.

Concerning the blower fan noise originating in the fan itself, evidence shows that thermophones with acceptable sizes are powerful enough to cancel this noise (Figure 10). Furthermore, if the it is likely that these thermophones will be able to achieve global cancellation of this particular source outside the ducting.

Moving on to the air rush noise within the ducting, the viability analysis becomes less clear. According to the model laid out in the previous section, the transducers must be as close to the duct exit as possible, and only air rush noise deeper within the ducting by some threshold will be cancellable. Because of this, it makes sense to consider each duct independently. This would mean placing a transducer group and controller at each duct 
outlet. In this case, the independent actuators would each be responsible for cancelling only the portion of the total air rush noise that corresponds to its own "cancellation zone" (Figure 93). Determining exactly how much sound these cancellation zones generate if considered independently is of critical importance. Consider the data collected from test trials of this experiment (Figure 95).

Broadband Cancellation vs. Disturbance Level (Ducting)

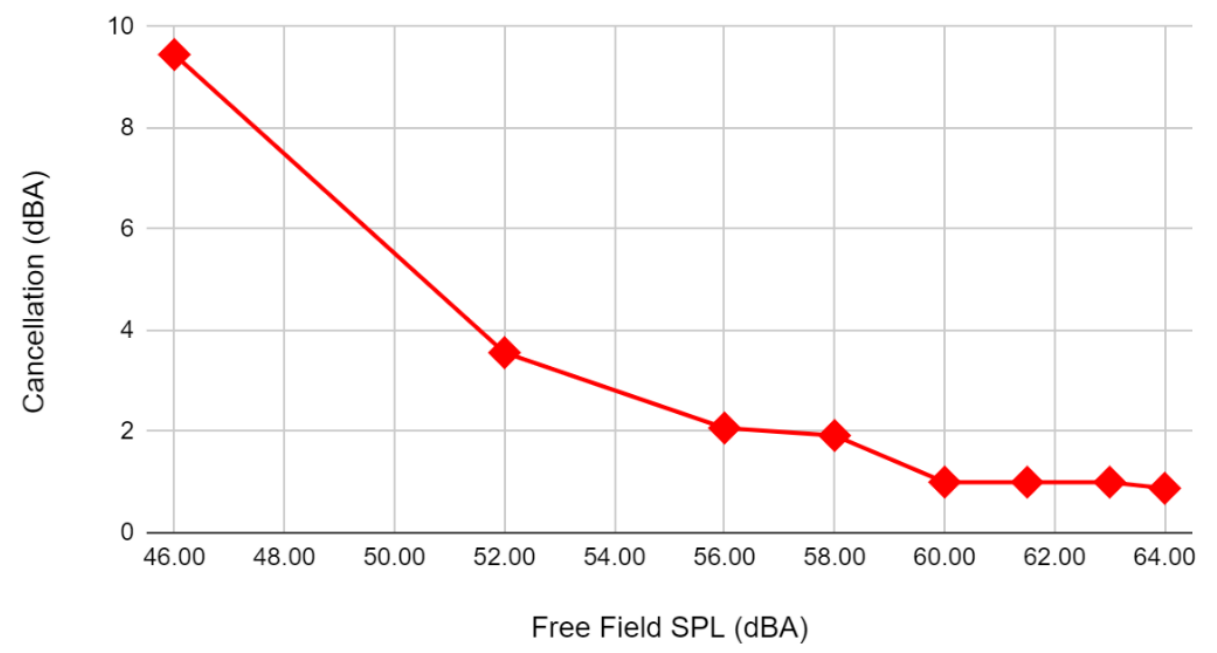

Figure 95: Cancellation Levels Achieved within a Ducted Environment for Disturbances which Varied in their pre-ANC SPL at $1 \mathrm{~m}$ from the duct outlet

Disturbances which only differed in their overall level before cancellation were generated with a loudspeaker in the ducted partition-mount setup (Figure 62). The actuator was supplied a constant power of $\sim 50 \mathrm{~W}$, and cancellation was attempted for all the trials using the Tiger WIFI-S. Cancellation was high where the overall disturbance level is low, and vice versa.

Using this, predictions as to the effectiveness of a $50 \mathrm{~W}$ thermophone for controlling air rush noise one of the independent ducts of the HVAC system are potentially possible. To do this, however, the level at which the occupant of a vehicle would experience noise emitted from just one ducting branch would need to be found. If this level were to be found, Figure 95 could be used to estimate the effectiveness of this actuator arrangement in cancelling sound from the first two source/path combinations.

In summation, from an acoustic output level perspective, thermophones seem to be viable as candidates for actuators which target globally coherent fan noise or air rush noise. For air rush noise it is necessary to place actuators at the end of every duct outlet, and it is likely that these actuators will be large in surface are. If a single duct from the HVAC could be characterized acoustically, it would be possible to determine the size of a CNT actuator which would be suitable for cancellation of air rush noise. 


\subsubsection{Acoustics - Sound Quality}

Based on the testing in this case study, CNT seems to perform marginally worse than traditional loudspeakers when used for ANC. For fan noise trials, CNT was expected to cancel roughly $1 \mathrm{~dB}$ less than loudspeakers. This is likely due to the amplitude modulation equipment used throughout the case study, which was constructed on a breadboard and not optimized for noise rejection.

In trials with non-fan disturbances it was found that the harmonic distortion of the thermophones had large potential to reduce cancellation performance. In tonal trials this harmonic distortion often caused a new tone to be introduced to the noise heard by an observer after ANC was applied. The same was true for broadband noise, but in some cases where the frequency range of the broadband noise overlapped with its own harmonics, the distortion was hidden by the original disturbance and remained unnoticed.

One strategy to reduce the overall levels of harmonics present in the output spectrum of the CNT is to decrease the modulation index of the amplitude modulation signal conditioning. Doing so, however, will reduce the efficiency of the thermophones. In other words, applying this strategy will mean a reduction in harmonics at the cost of thermophone efficiency.

It has been shown in the case study that CNT actuators can work almost as well as loudspeaker actuators, but that the system designer needs to pay careful attention to the presence of harmonic distortion in the output spectra of the thermophones, and come up for a plan to deal with them in a way that suits the system.

\subsubsection{Form Factor}

Since this has been touted as one of the main advantages of CNT thermophones, even before this case study began, it is no surprise that the thermophones earn high marks here. Specifically, the ability of the thermophones to be contained within the duct geometry, such as in the case of FC01 or the annular Teflon thermophone, is beneficial according to the model from the prior section. Installing traditional loudspeakers in ducts for ANC purposes typically relies on a separate branch of ducting which is added to the system specifically for this purpose. The elimination of this extra branch simplifies the ducting system, and decreases the microphone spacing required for a causal system since sound must travel through this section to get to the error microphone no longer. As shown in the paragraphs surrounding Figure 93, this is beneficial in maximizing the theorized "cancellable zone" of this model.

\subsubsection{Temperature}

Due to the fundamental inefficiency of the thermoacoustic effect, all thermophones produce a large amount of waste heat. In an HVAC environment that is almost all plastic, this can pose serious problems if not managed properly. Luckily, fast moving air is also 
abundantly present in HVAC systems. Testing throughout the case study showed that thermophones installed into the blower fan would not bring the plastic fan material above safe working temperatures, provided the fan was running (Figure 12, Figure 13, Figure 19, Figure 23, \& Figure 30). If for whatever reason, the thermophone continues to operate while the fan does not, temperatures will exceed safe working limits very quickly (Figure 31).

To conclude, the integration of thermophones into the HVAC system seems feasible and has been demonstrated as working from a temperature perspective. Great care must be taken, however, to create, a robust failsafe which prevents the thermophones from operating while the HVAC system is off. A graveyard of melted 3D printed thermophone parts in the MTU acoustics lab will attest to this.

\subsubsection{Durability}

Described in the CNT primer as "a wisp of smoke," CNT thin film in its raw form is extremely delicate. Luckily, thermophone designs can work around this, and protection of the fragile film is possible, even in very high airflow. Both rigid CNT panel and flexible designs demonstrated this in the HVAC system, granted, under laboratory conditions. Not much else can be reported in terms of CNT durability viability other than observations that some thermophone designs ( $\mathrm{RC01}$ especially) protected the CNT excellently throughout all tests performed.

\subsubsection{Cost and Manufacturability}

These metrics for CNT are the most unknown. Factories producing the type of CNT used to make thermophones at commercial scales do not exist. Currently MTU purchases its CNT from a lab at the University of Cincinnati at a price that is well above what would be economically feasible for the automotive supply market. However, the materials used to produce CNT are abundant and easy to acquire, and the production process is scalable [64] [65]. Until demand for CNT reaches sufficient levels, true cost predictions cannot be made accurately, at least by MTU, so no further comments will be given.

Mass manufacturability of thermophones also falls into this category. The material is fragile and difficult to work with by hand. Devising a manufacturing line which could solve these problems and produce thermophones at a high rate is well out of the wheelhouse of the authors of this report. That does not mean, however, that it cannot be done. Additional studies concerning this would need to be performed before further comments can be made. 


\section{Conclusions \& Future Work}

This case study investigated the union of ANC and CNT thermophone technology for use in reducing overall noise levels from an automotive HVAC. Throughout the 18 months of research dedicated to this, many victories, defeats, stagnation points, and breakthroughs were experienced by the development team. Like any research project which starts out hoping to determine the feasibility of fledgling technologies in a new environment, the scope of the case study became more and more focused as time progressed. Initial hopes of producing a functioning ANC system which reduced broadband HVAC noise by $10 \mathrm{~dB}$ overall at the end of the project period were ultimately drowned out by the complexity of the system. That is not to say the case study was not valuable, however.

The broad, system-wide goals of the case study onset were replaced by more focused ones that targeted individual system pieces. In this respect, much progress was made. First, a clear division of the overall system into three source/path pairs which should be considered from an ANC design perspective was proposed. Details of the acoustic nature of two of these were also investigated, and a theory to explain their behavior in the HVAC system was laid out.

Of equal importance to the theoretical work done in this study were the design and testing threads. Several prototypes of thermophone configurations which could be installed in ducting/HVAC systems were built. Analysis of the performance of these thermophones was also done, and evidence shows that CNT thermophones could be viable ANC actuators for use in reducing the levels of one of the three proposed source/path pairs in an auto HVAC. Both the RC01 and RC03 thermophone prototypes were shown to be able to match the level of the globally-coherent fan noise propagating through the ducting in the system, meaning that they could potentially cancel this source for the passengers of the vehicle.

Unfortunately, it was found that this source was not the dominant source in the overall noise heard by the passengers. Air rush noise propagating through the ducting dominated instead. The design of an ANC system with the best chance at cancelling air rush was developed and realized. Once built, the system showed that at its current level of development, ANC in general is not ready to reduce the overall level of air rush noise propagating through ducting. In an HVAC where this is the dominant source heard by an observer, this also means that overall level changes in the HVAC noise are not possible through the application of ANC. In a different HVAC, however, with a dominating bladepass frequency or fan rumble, ANC could provide a good noise control solution.

With the proposed optimal system proven not to be functional enough for use in ducted ANC design, future work would do well to focus on improving this system. The main subject of investigations should be finding the difference between techniques for acquiring the reference signal. Broadband performance sees significant increases when a direct line to the disturbance source is used as the reference instead of a microphone. There is some difference between the characteristics of these two signals which allows the algorithm to find a solution in one case but not the other. Once this question is answered, a solution 
can be determined which may allow a microphone to serve as an equally viable reference transducer. 


\section{References}

[1] K. Jiang, J. Wang, Q. Li, L. Liu, C. Liu, and S. Fan, "Superaligned Carbon Nanotube Arrays, Films, and Yarns: A Road to Applications," vol. 23, no. 9, pp. 1154-1161, 2011, doi: doi:10.1002/adma.201003989.

[2] M. Zhang et al., "Strong, transparent, multifunctional, carbon nanotube sheets," Science (New York, N.Y.), vol. 309, no. 5738, p. 1215, 2005, doi: 10.1126/science.1115311.

[3] K. Jiang, Q. Li, and S. Fan, "Nanotechnology: spinning continuous carbon nanotube yarns," (in eng), Nature, vol. 419, no. 6909, p. 801, Oct 24 2002, doi: 10.1038/419801a.

[4] Y. Wei, L. Liu, P. Liu, L. Xiao, K. Jiang, and S. Fan, "Scaled fabrication of singlenanotube-tipped ends from carbon nanotube micro-yarns and their field emission applications," Nanotechnology, vol. 19, no. 47, p. 475707, 2008/10/30 2008, doi: 10.1088/0957-4484/19/47/475707.

[5] P. Liu, Y. Wei, L. Liu, K. Jiang, and S. Fan, "Formation of free-standing carbon nanotube array on super-aligned carbon nanotube film and its field emission properties," Nano Research, vol. 5, no. 6, pp. 421-426, 2012/06/01 2012, doi: 10.1007/s12274-012-0224-3.

[6] G. A. Mansoori, T. F. George, L. Assoufid, and G. Zhang, Thermal Properties of Carbon Nanotubes (Topics in Applied Physics). New York, NY: Springer New York, 2007, pp. 154-187.

[7] W. Yi, L. Lu, Z. Dian-lin, Z. W. Pan, and S. S. Xie, "Linear specific heat of carbon nanotubes," Physical Review B, vol. 59, no. 14, pp. R9015-R9018, 04/01/1999, doi: 10.1103/PhysRevB.59.R9015.

[8] L. Xiao et al., "Flexible, Stretchable, Transparent Carbon Nanotube Thin Film Loudspeakers," Nano Letters, vol. 8, no. 12, pp. 4539-4545, 2008/12/10 2008, doi: $10.1021 / \mathrm{nl} 802750 z$.

[9] F. Braun, Ann der Physik, vol. 65, 1898.

[10] H. D. Arnold and I. B. Crandall, "The Thermophone as a Precision Source of Sound," Physical Review, vol. 10, no. 1, pp. 22-38, 07/01/ 1917, doi: 10.1103/PhysRev.10.22.

[11] C. Lim, L. Tong, Y. J. J. o. S. Li, and Vibration, "Theory of suspended carbon nanotube thinfilm as a thermal-acoustic source," vol. 332, no. 21, pp. 5451-5461, 2013.

[12] A. E. Aliev et al., "Thermal management of thermoacoustic sound projectors using a free-standing carbon nanotube aerogel sheet as a heat source," vol. 25, no. 40, p. 405704, 2014. [Online]. Available: http://iopscience.iop.org/article/10.1088/0957-4484/25/40/405704/pdf.

[13] T. M. Bouman, A. R. Barnard, and M. Asgarisabet, "Experimental quantification of the true efficiency of carbon nanotube thin-film thermophones," The Journal of the Acoustical Society of America, vol. 139, no. 3, pp. 1353-1363, 2016/03/01 2016, doi: $10.1121 / 1.4944688$.

[14] T. Bouman, "DRIVE SIGNAL DEVELOPMENT FOR THE THERMOACOUSTIC LOUDSPEAKER," Michigan Technological University, Houghton, Michigan, 2016.

[15] M. E. Hawley, "Acoustic Interference for Noise Control," Noise Control, vol. 2, no. 2, pp. 61-94, 1956/03/01 1956, doi: 10.1121/1.2369191. 
[16] B. Pinter, "Active noise-cancelling headphones," ed: Google Patents, 2020.

[17] Y. Xing, "Helicopter cockpit active noise control system," ed, 2018.

[18] P. Loiseau, P. Chevrel, M. Yagoubi, and J.-M. Duffal, "Robust active noise control in a car cabin: Evaluation of achievable performances with a feedback control scheme," Control Engineering Practice, vol. 81, pp. 172-182, 2018, doi: 10.1016/j.conengprac.2018.09.015.

[19] J. Cheer and S. J. Elliott, "Active noise control of a diesel generator in a luxury yacht," Applied Acoustics, vol. 105, pp. 209-214, 2016, doi:

10.1016/j.apacoust.2015.12.007.

[20] J. Romeu Garbí, J. Palacios, A. Balastegui Manso, and T. Pàmies Gómez, "Optimization of the active control of turboprop cabin noise," ed, 2015.

[21] J. Wang, L. Huang, and L. Cheng, "A study of active tonal noise control for a small axial flow fan," The Journal of the Acoustical Society of America, vol. 117, no. 2, pp. 734-743, 2005/02/01 2005, doi: 10.1121/1.1848072.

[22] N. Devineni, I. Panahi, and P. Kasbekar, "Predictive multi-channel Feedback active noise control for HVAC systems," in 2011 IEEE INTERNATIONAL CONFERENCE ON ELECTRO/INFORMATION TECHNOLOGY, 15-17 May 2011 2011, pp. 1-5, doi: 10.1109/EIT.2011.5978581.

[23] P. Kasbekar, A. Wisler, and I. M. S. Panahi, "A two-channel feedback active noise control system with delayless subband architecture to reduce compressor noise of an enclosed heating, ventilation and air conditioning unit," Journal of Vibration and Control, vol. 21, no. 7, pp. 1430-1442, 2015, doi: 10.1177/1077546313499058.

[24] A. P. Patwardhan, R. Patidar, and N. V. George, "Dynamic nonlinear active noise control: A multi-objective evolutionary computing approach," in Nature-Inspired Methods for Metaheuristics Optimization: Springer, 2020, pp. 421-439.

[25] P. Li, X. Bai, and Y. Ma, "Two kinds of active impulsive noise control algorithms based on sigmoid transformation," in Seventh International Conference on Electronics and Information Engineering, 2017, vol. 10322: International Society for Optics and Photonics, p. 103220R.

[26] J. Zhang, T. D. Abhayapala, W. Zhang, P. N. Samarasinghe, and S. Jiang, "Active noise control over space: A wave domain approach," IEEE/ACM Transactions on audio, speech, and language processing, vol. 26, no. 4, pp. 774786, 2018.

[27] K. Chen and J. Lu, "Kalman filter based active noise control algorithm with simultaneous transfer function modeling," in INTER-NOISE and NOISE-CON Congress and Conference Proceedings, 2018, vol. 258, no. 5: Institute of Noise Control Engineering, pp. 2112-2120.

[28] D. R. Morgan, "History, applications, and subsequent development of the FXLMS Algorithm [DSP History]," IEEE Signal Processing Magazine, vol. 30, no. 3, pp. 172-176, 2013, doi: 10.1109/MSP.2013.2242394.

[29] S. M. Kuo and D. R. Morgan, "Review of DSP algorithms for active noise control," ed: IEEE, 2000, pp. 243-248.

[30] C. Hansen, S. Snyder, X. Qiu, L. Brooks, and D. Moreau, Active Control of Noise and Vibration. 2012.

[31] S. G. Hill, N. Tanaka, and H. Iwamoto, "Local sensing/control or global error sensing/control in structural acoustics: A comparison of two techniques influence over sound power," Applied Acoustics, vol. 71, no. 10, pp. 965-978, 2010, doi: 10.1016/j.apacoust.2010.06.008. 
[32] J. Pan, "Local or global control - The application active noise control strategy in enclosed environments," INTER-NOISE and NOISE-CON Congress and Conference Proceedings, vol. 2002, no. 7, pp. 93-105, 2002.

[33] S. P. Mavuri, S. Watkins, X. Wang, S. St. Hill, and D. Weymouth, "An Investigation of Vehicle HVAC Cabin Noise," ed, 2008.

[34] N. Humbad, "Automotive HVAC Noise Measurements and Analysis," Chinese Journal of Automotive Engineering, vol. 1, no. 4, pp. 299-309, 2011, doi: 10.3969/j.issn.2095-1469.2011.04.007.

[35] "ASTM E2611-19, Standard Test Method for Normal Incidence Determination of Porous Material Acoustical Properties Based on the Transfer Matrix Method," ASTM International, West Conshohocken, PA, 2019, doi: 10.1520/E2611-19.

[36] S. M. Kuo and D. R. Morgan, "Active Noise Control Systems: Algorithms and DSP Implementations," 1996.

[37] S. Kuo, M. Nadeski, T. Horner, J. Chyan, and I. Panahi, "Fixed-point DSP implementation of active noise control systems," The 1994 National Conference on Noise Control Engineering, Fort Lauderdale, FL, USA, 05/01-04/94, 1994.

[38] M. Kuo Sen, "Active noise control system and method for on-line feedback path modeling," 7 ed, 2002.

[39] M. T. Akhtar, M. Abe, and M. Kawamata, "On Active Noise Control Systems With Online Acoustic Feedback Path Modeling," IEEE Transactions on Audio, Speech, and Language Processing, vol. 15, no. 2, pp. 593-600, 2007, doi:

10.1109/TASL.2006.876749.

[40] M. Akhtar, M. Tufail, M. Abe, and M. Kawamata, "Acoustic feedback neutralization in active noise control systems," leice Electronics Express, vol. 4, no. 7, pp. 221-226, 2007, doi: 10.1587/elex.4.221.

[41] G. J. Miao, Signal processing in digital communications (Artech House signal processing library). Boston, MA: Artech House, 2007.

[42] A. Aguilar-González, M. Arias-Estrada, M. Pérez-Patricio, and J. CamasAnzueto, "An FPGA 2D-convolution unit based on the CAPH language," Journal of Real-Time Image Processing, vol. 16, no. 2, pp. 305-319, 2019, doi: 10.1007/s11554-015-0535-1.

[43] "NI 9234 Datasheet," N. Instruments, Ed., ed: National Instruments, 2015.

[44] P. Babu and A. Krishnan, "A New Variable Threshold and Dynamic Step Size Based Active Noise Control System for Improving Performance," arXiv.org, 2010.

[45] N. Thai, X. Wu, J. Na, Y. Guo, and P. Le, "Adaptive active noise control with online secondary path modelling and variable step-size learning," International Journal of Modelling, Identification and Control, vol. 25, no. 2, pp. 71-84, 2016, doi: 10.1504/IJMIC.2016.075278.

[46] W.-S. Gan, "Fuzzy step-size adjustment for the LMS algorithm," Signal Processing, vol. 49, no. 2, pp. 145-149, 1996, doi: 10.1016/0165-1684(96)000394.

[47] H. Boyan, X. Yegui, S. Jinwei, and W. Guo, "A Variable Step-Size FXLMS Algorithm for Narrowband Active Noise Control," IEEE Transactions on Audio, Speech, and Language Processing, vol. 21, no. 2, pp. 301-312, 2013, doi: 10.1109/TASL.2012.2223673.

[48] N. kunchakoori, A. Routray, and D. P. Das, "An Energy Function Based Fuzzy Variable Step Size FxLMS Algorithm for Active Noise Control," in 2008 IEEE Region 10 and the Third international Conference on Industrial and Information Systems, 8-10 Dec. 2008 2008, pp. 1-7, doi: 10.1109/ICIINFS.2008.4798476. 
[49] V. Madani, "Noise Sources Characterization of Automotive HVAC Systems," MS Mechanical Engineering, McMaster University, 2002.

[50] S. Bennouna, T. Matharan, and O. Cheriaux, "Automotive HVAC Noise Reduction," ed, 2018.

[51] S. Bennouna, S. Naji, O. Cheriaux, S. Moreau, B. Ouedraogo, and J. M. Ville, "Aeroacoustic prediction methods of automotive HVAC noise," vol. 2015-, ed: SAE International, 2015.

[52] A. McAlpine, A. P. Daymond-King, and A. Kempton, "Sound radiation from a flanged inclined duct," Journal Of The Acoustical Society Of America, vol. 132, no. 6, pp. 3637-3646, 2012, doi: 10.1121/1.4764883.

[53] K. Tanaka, Y. Tsubakishita, and K. Hayashi, "Acoustic field around an exit of flanged cylindrical duct with a coaxial cavity," Jsme International Journal Series B-Fluids And Thermal Engineering, vol. 41, no. 3, pp. 618-623, 1998.

[54] R. Kirby and W. Duan, "Sound radiation from the open end of pipes and ducts in the presence of mean flow," 2017.

[55] L. Zhang and X. Qiu, "Causality study on a feedforward active noise control headset with different noise coming directions in free field," Appl. Acoust., vol. 80, p. 36, 2014.

[56] J. Ma, H. Zou, and X. Qiu, "Performance analysis of feedforward active noise control systems with acoustic feedback," Shengxue Xuebao/Acta Acustica, vol. 41, no. 5, pp. 686-693, 2016.

[57] K.-C. Chen, C.-Y. Chang, and S. M. Kuo, "Active noise control in a duct to cancel broadband noise," IOP Conference Series: Materials Science and Engineering, vol. 237, p. 012015, 2017/09 2017, doi: 10.1088/1757-899x/237/1/012015.

[58] M. Larsson, "Active Noise Control in Ventilation Systems: Practical Implementation Aspects," ed. Karlskrona: Blekinge Institute of Technology, 2008.

[59] S. G. Boroujeny and M. Eshghi, "FPGA implementation of a modular active noise control system," in 2010 18th Iranian Conference on Electrical Engineering, 11-13 May 2010 2010, pp. 658-661, doi: 10.1109/IRANIANCEE.2010.5506991. [Online]. Available: https://ieeexplore.ieee.org/document/5506991/

[60] M. Larsson, S. Johansson, L. Håkansson, and I. Claesson, "Microphone Windscreens for Turbulent Noise Suppression when Applying Active Noise Control to Ducts," ed. Lisbon, Portugal: International Institute of Acoustics and Vibration, 2005.

[61] R. McGuinn, G. Lauchle, and D. Swanson, "Low flow-noise microphone for active noise control applications," AIAA Journal, vol. 35, no. 1, pp. 29-34, 1997, doi: 10.2514/2.83.

[62] F. Ducret, "Studies of sound generation and propagation in flow ducts," ed, 2006.

[63] M. Larsson, K. Nilsson, S. Johansson, I. Claesson, and L. Håkansson, "An Active Noise Control Approach for Attenuating Noise Above the Plane Wave Region in Ducts," ed. Rio de Janeiro, Brazil: International Institute of Acoustics and Vibration (IIAV), 2011.

[64] J. Logeswari, A. Pandurangan, and D. Sangeetha, "An Efficient Catalyst for the Large Scale Production of Multi-Walled Carbon Nanotubes," Industrial \& Engineering Chemistry Research, vol. 50, no. 23, pp. 13347-13354, 2011/12/07 2011, doi: 10.1021/ie102525u.

[65] A. Morançais et al., "A parametric study of the large scale production of multiwalled carbon nanotubes by fluidized bed catalytic chemical vapor deposition," Carbon, vol. 45, no. 3, pp. 624-635, 2007/03/01/ 2007, doi:

https://doi.org/10.1016/i.carbon.2006.10.009. 


\section{A. Design and Equipment Gallery}

\section{Thermophones \& Loudspeakers}

\section{Rigid CNT Panel}

This isn't really a very useful thermophone in itself, but it makes an appearance at many stages throughout the project in some way shape or form. A rigid CNT panel is constructed with three components: a rigid substrate, an electrode pair, and CNT thin film (Figure 96).
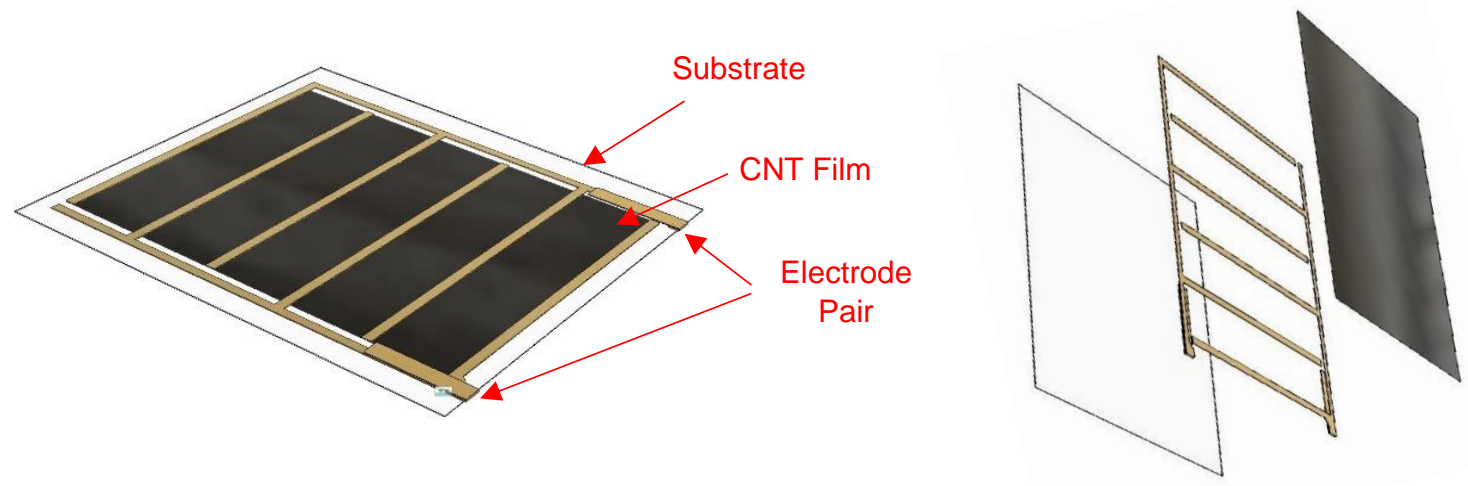

Figure 96: Rigid CNT Panel Renderings - Callout and Exploded Views

The electrode pair is cut from a conductive material to the desired pattern. Typically, copper tape is used and is cut into two comb patterns so that the "teeth" of the two electrodes interleave. By changing the width and spacing of the electrodes the resistance of the speaker can be tuned to match an amplifier. The electrode pair is then adhered to the substrate material. This substrate must be highly heat resistant and electrically insulating. Typical materials include ceramic plates, glass, and select Teflon and fiberglass plates. The CNT is then pulled over the electrodes, perpendicular to the direction of the individual electrode "teeth." Any number of layers of CNT can be applied, but 5 is typically a good number 


\section{Flexible CNT Composite}

The flexible thermophone variants all take their core design from the composite described here. The layer makeup is very similar to that of the rigid CNT panel with two key differences: the substrate must be flexible here, and an additional substrate layer is added. First a layer of substrate has electrode patterns adhered to it. Then, CNT is stretched over the substrate / electrodes. Strips of adhesive are then applied as close to the CNT as possible. It is useful to divide the CNT into "cells" so that the adhesive strips aren't spaced very far apart. Finally, the last layer of substrate is stretched over the whole composite, sealing the CNT into the middle of a multilayer composite. The image below shows a composite that is complete except for the application of the final substrate layer.

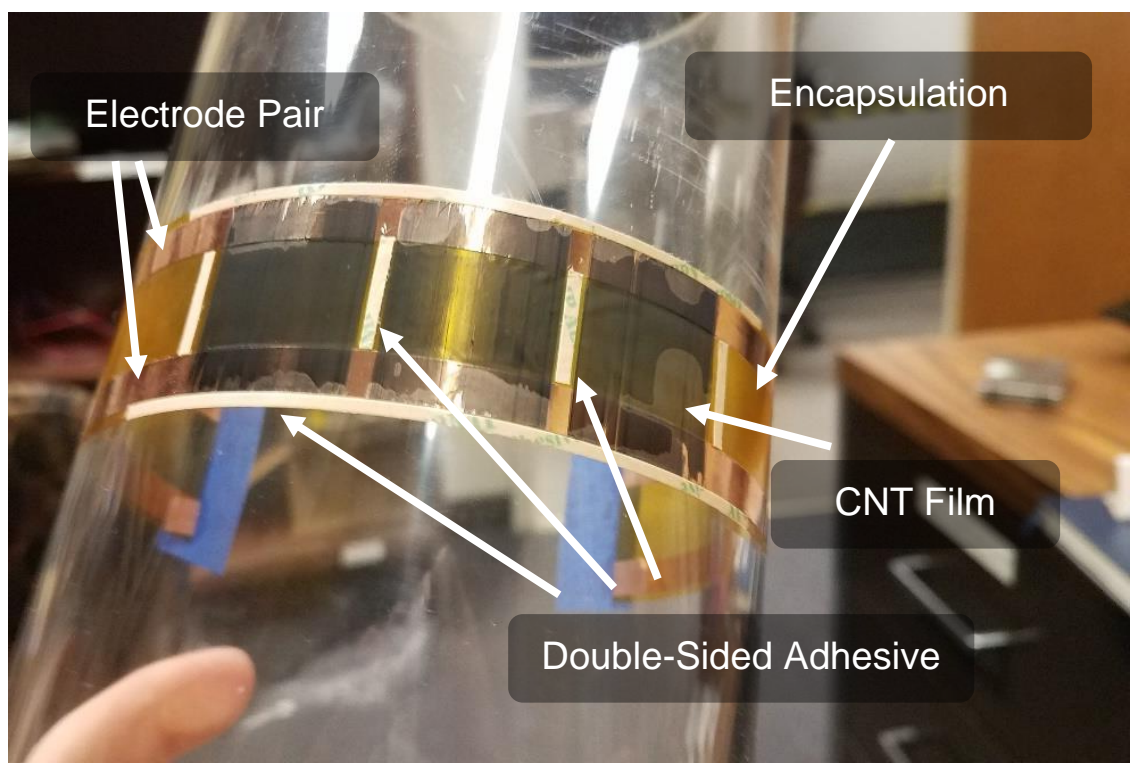

Figure 97: A Four-Layer Composite CNT Thermophone Before Application of the Final Encapsulation Layer
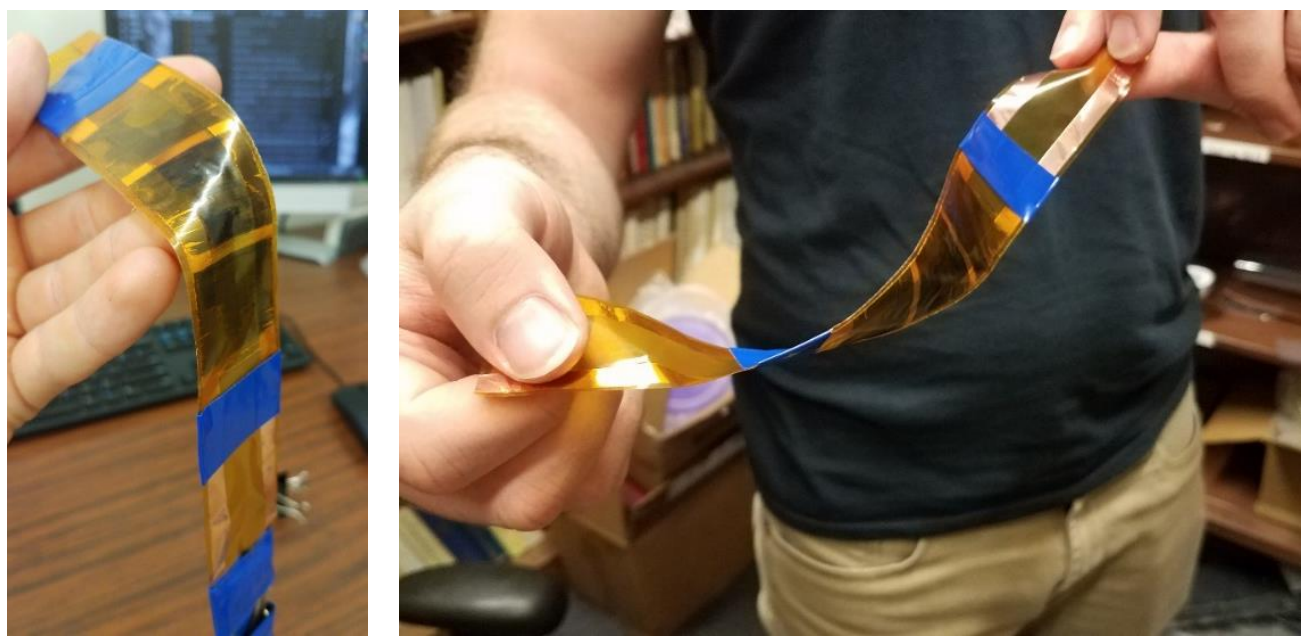

Figure 98: Completed Four-Layer Composite CNT Thermophone using Kapton Film for Encapsulation 


\section{Rigid Concept 01 (RC01) Prototype}

This prototype was the first attempt at installing a CNT speaker into the HVAC system. It took the form of a cowling that was glued into place on the interior surface of the blower fan, near the exhaust port. With a closed geometry on the upstream side and an opening downstream, the cowling allowed the CNT to be protected from airflow while still emitting sound out into the exhaust of the fan. Physically the cowling was made of a 3D printed PETG plastic, into which a rigid CNT panel made from glass was installed (Figure 99).
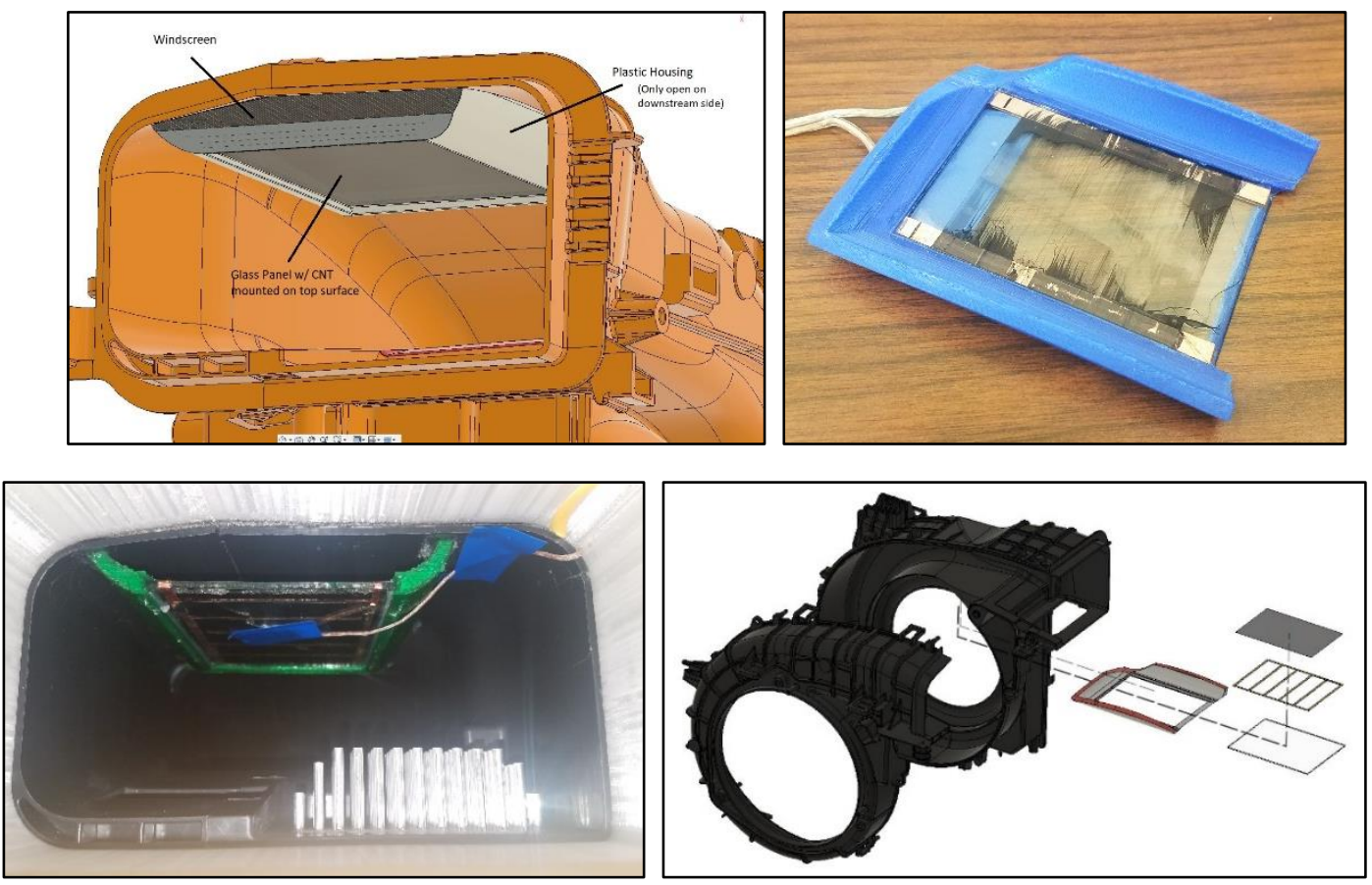

Figure 99: Four Different Representations of the RC01 Prototype Thermophone

The rigid CNT panel had an overall CNT area (5 layers) of $42 \mathrm{~cm}^{2}$ and was designed for a resistance of $8 \Omega$. While RC01 was very successful in terms of its CNT protection, acoustic performance, and thermal characteristics, it was ultimately scrapped. Because it caused a reduction in exhaust outlet cross-sectional area the design was deemed unacceptable from an airflow perspective early in the project. Not much beyond simple acoustic and thermal testing was performed on this design. 


\section{Rigid Concept 03 (RC03) Prototype}

RC03 was the second rigid prototype concept to be realized and installed inside the blower fan. It is similar to the RC01 speaker, the main difference between the two being the location of the cowling geometry. Where the cowling of RC01 protruded into the airflow path significantly, RC03 was effectively mirrored about the exhaust's boundary surface. The chamber housing the CNT was outside the fan, and sound was emitted into the airstream through a small "acoustic slot" that connected the chamber to the airflow path (Figure 100).
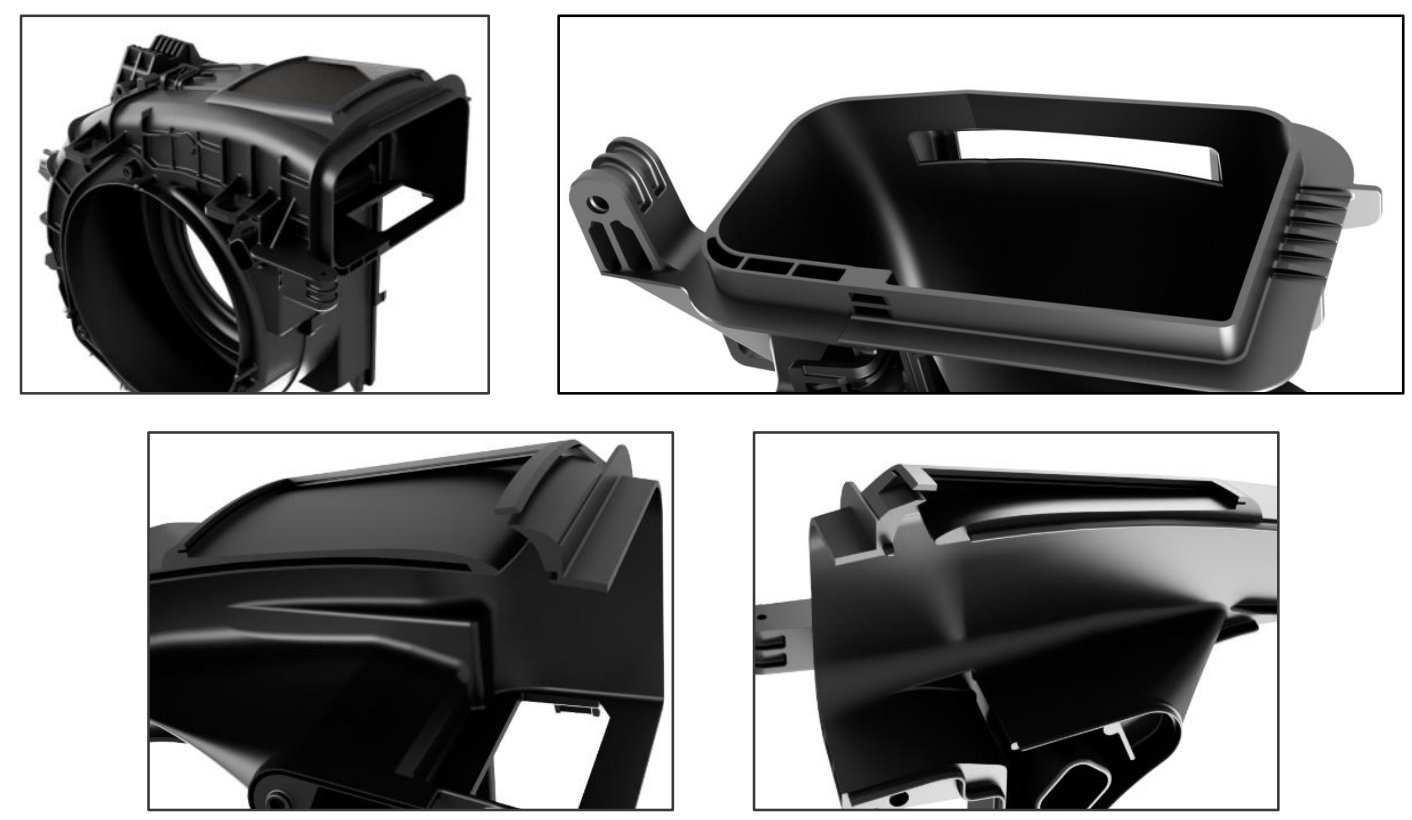

Figure 100: Renderings of the Initial RC03 Thermophone Prototype Geometry Integrated into the Blower Fan

Since this geometry is more complicated than RC01 a custom 3D-printed version of the blower fan casing was made by the project sponsor and provided to MTU. Originally, a thin bendable glass called Willow Glass was going to be used as the substrate for the CNT panel in this design. This material proved too delicate to work with and was replaced by eventually a fiberglass panel, which was installed on top of the curved CNT chamber by means of an adapter bezel. This configuration of the prototype was the one used during most of the tonal ANC testing (Figure 101). 


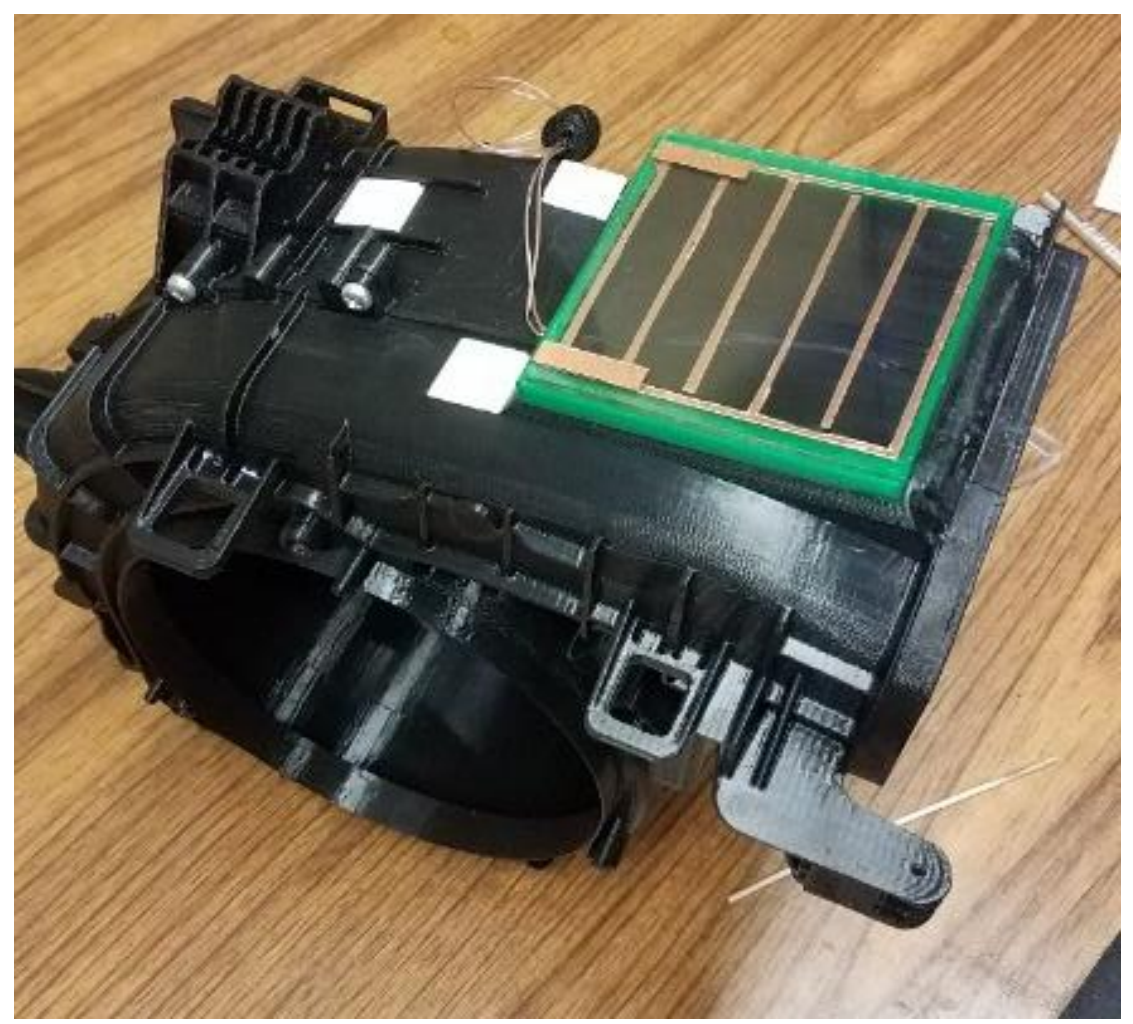

Figure 101: Glass Rigid CNT Panel Installed onto RC03 Prototype Thermophone Using a 3D-Printed Adapter 


\section{Flexible Concept 01 (FC01) Prototype}

FC01 was the third major prototype iteration to be installed into the HVAC system. It was designed and built following the four-layer composite template after fiberglass fabric was identified as the best encapsulation material. Dimensions of the speaker are $70 \mathrm{~cm} x 8 \mathrm{~cm}$, and the electrodes are configured for $8 \Omega$ resistance.
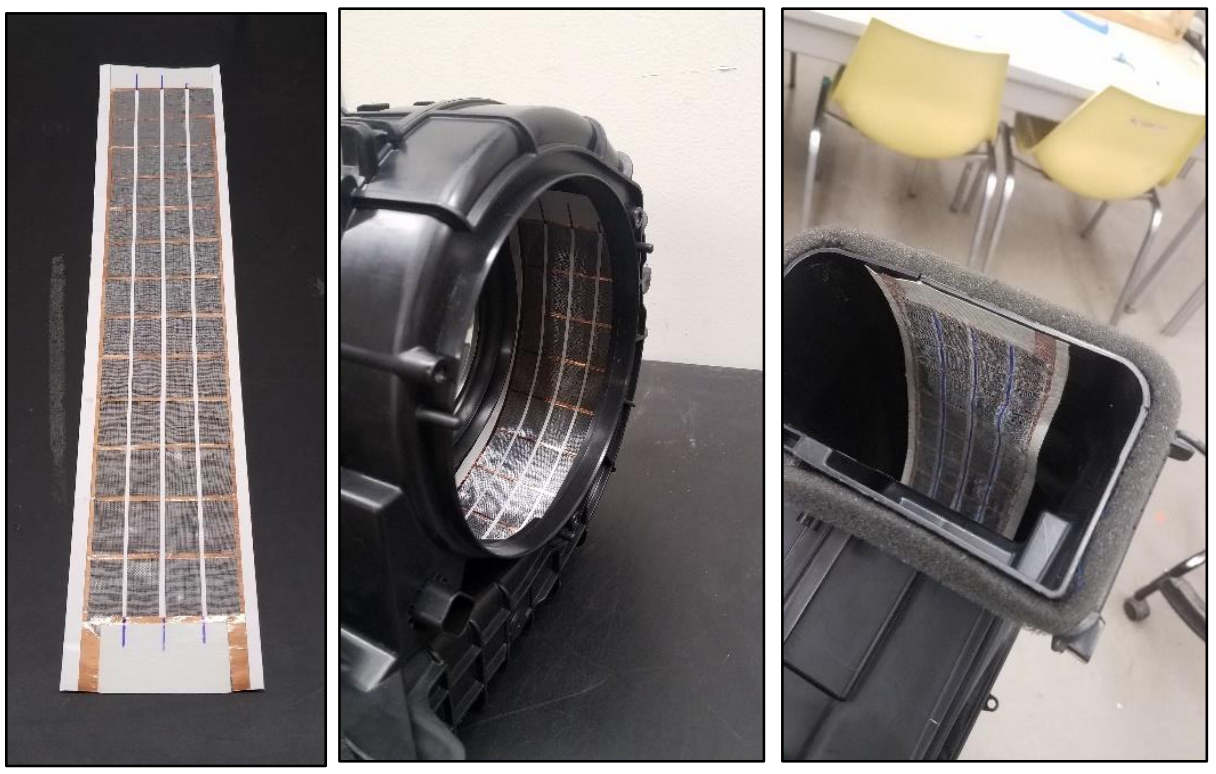

Figure 102: FC01 Thermophone Prototype Before and After Installation into Blower Fan

At the point in time that FC01 was designed and built a high-temperature double-sided adhesive for sealing the speaker together had still not been found. Thin carpet tape was used for this purpose instead. Because of this, the round of fiberglass speakers built around this time (including FC01), would emit chemical odors from adhesive melt if allowed to overheat. Despite this, the actual durability of the FC01 prototype was good, and after installation the speaker did not show major signs of degradation throughout its use. As the speaker was being installed however, damage was done to some of the CNT cells. After suspecting this to be the case due to the extreme bending required to get the speaker all the way into the fan a thermal camera was used to inspect the CNT while it was running. Doing so allows observation of which cells are and are not activating. The thermal camera revealed that all cells were not operating, and that moving the composite speaker slightly by hand, pressing the electrodes back into the CNT, could activate thes cells.

While the post-installation durability of the FC01 prototype seemed to be good, the acoustic output and thermal properties of the design were subpar. Output levels of FC01 were found to be less than those of RC03. Thermal performance while the fan was running was acceptable but cutting fan power would cause the speaker to overheat in a matter of seconds after airflow was stopped. 


\section{Annular Teflon Thermophone}

During the tail end of the case study it became clear it would be more valuable to focus testing on the controller and ANC in a general sense than on the HVAC system. With this purpose in mind, a durable and versatile CNT speaker was designed. Built to fit into two different general-purpose test setups, this speaker takes the shape of an annulus. This is particularly advantageous in round ducting, where it can form a plane wave instantly at certain frequencies. Specifically, this speaker was designed to slide snugly into 4" PVC, which could then be fit onto the end of the blower fan. In addition to serving as the actuator in single duct, high-airflow environments, the speaker also fit onto a special stand for freefield cancellation trials (Figure 103).
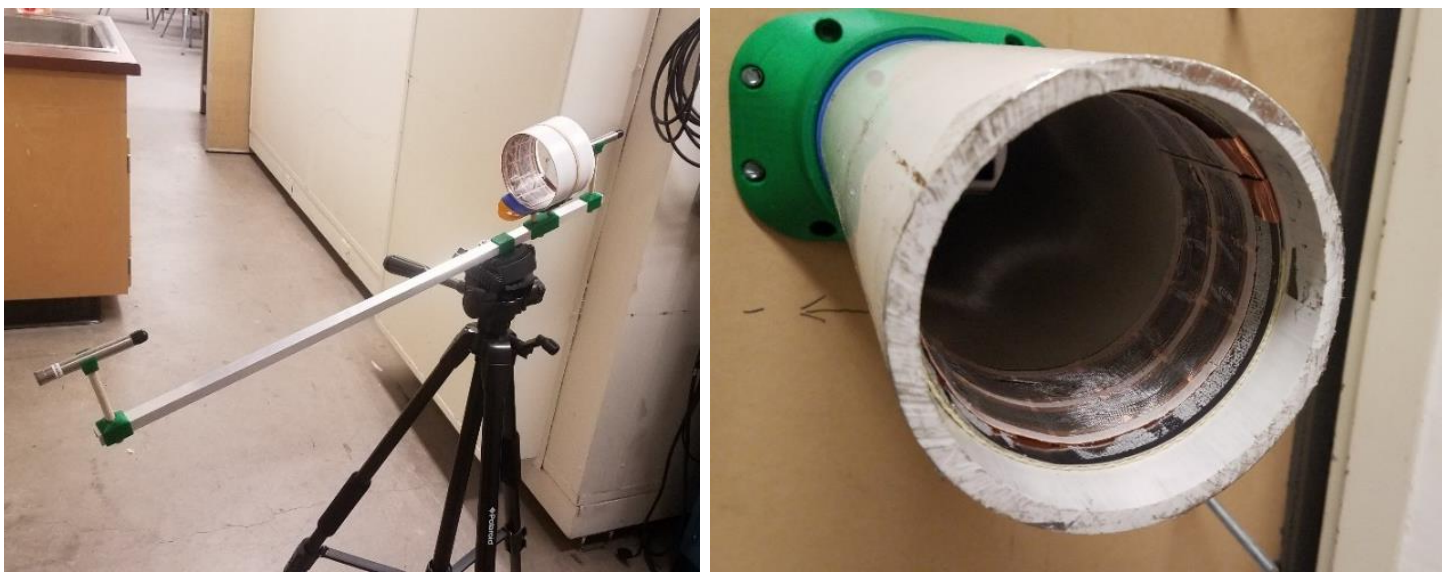

Figure 103: Annular Teflon Speaker in Two Test Configurations

The construction of the speaker relies on first creating a ring of PTFE material. 1/8" PTFE sheets were used, cut into strips $\sim 3$ " wide. The ends of the strip were secured to a small extension piece using two lap joints. To secure the lap joints a high-temperature doublesided silicone/PTFE adhesive was used. Once the ring was created a four-layer composite thermophone was built and applied to the inner ring surface (Figure 104). 


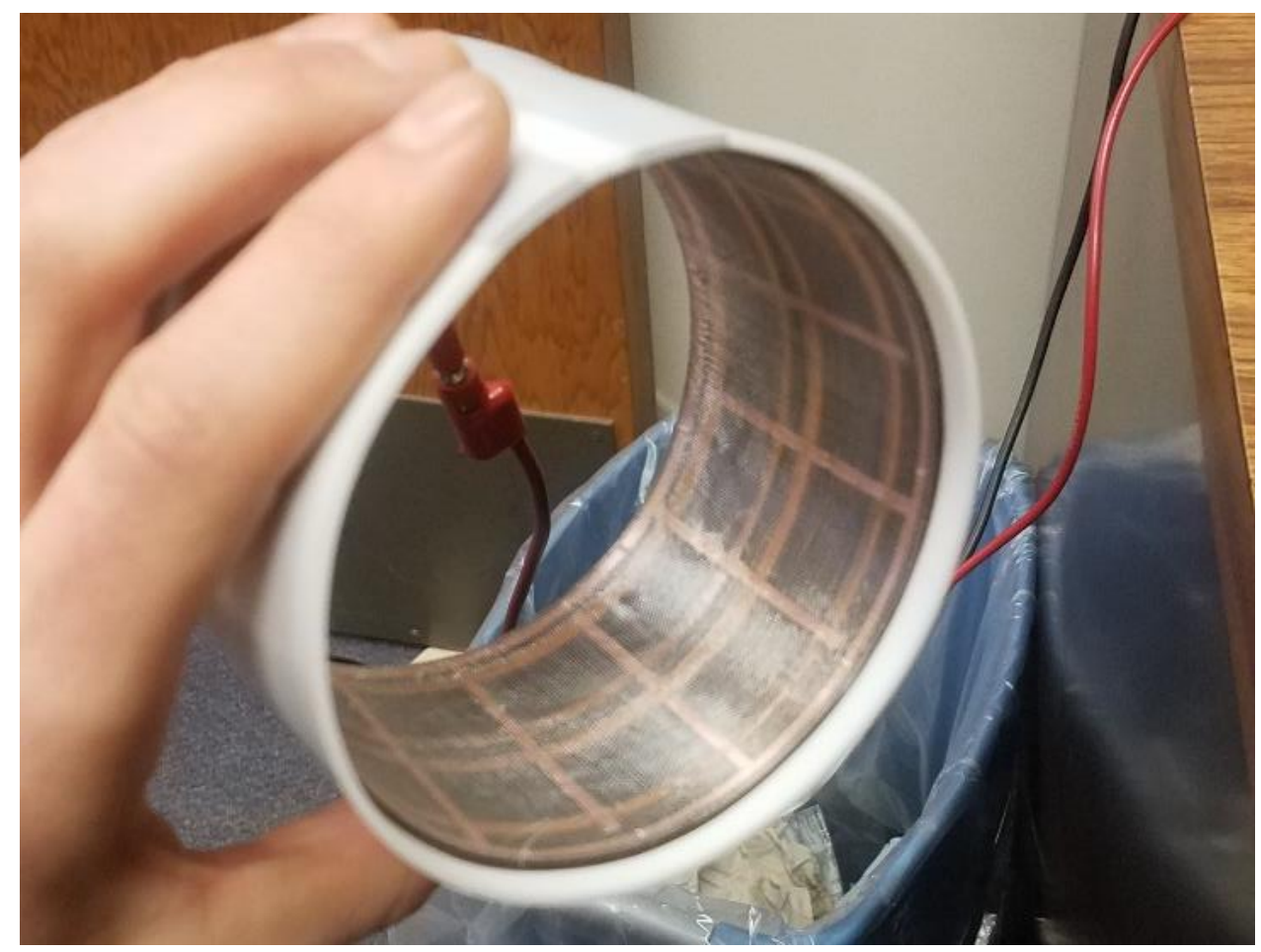

Figure 104: Close up of Annular Teflon Speaker Construction 


\section{Annular Aluminum Thermophone}

Once enough information was gathered from free-field testing, all attention was shifted towards in-duct testing. Because of this the flexibility of the Annular Teflon thermophone was no longer required. A new "ducting-only" thermophone that eliminated some of the disadvantages of the Annular Teflon thermophone was developed.

The annular Aluminum thermophone combines the design concepts of the RC01 prototype and the Annular Teflon thermophone, using a cowling-type CNT enclosure which is shaped like an annulus. A thin-walled tube of aluminum with an outer diameter of roughly 3.5" serves as the rigid CNT panel. A layer of Kapton film is applied to the outside of this tube, then electrodes and CNT are applied on top of the Kapton. The ends of the tube are then fitted with bushings so that it can slide concentrically into the 4" PVC duct. The upstream bushing is closed to flow and is tapered to make the transition between inner diameters gradual. The downstream bushing is open to flow so that sound can radiate out of the CNT chamber and into the ducting.

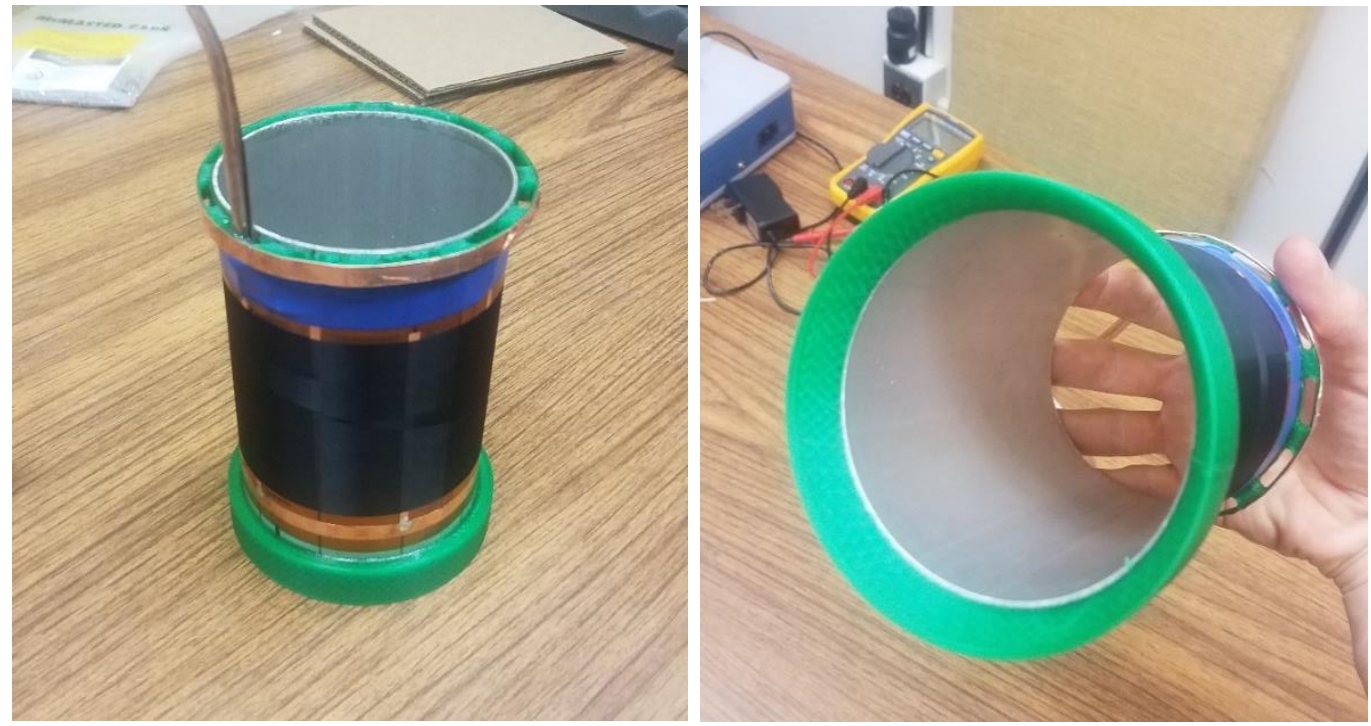

Figure 105: Construction of the Annular Aluminum Thermophone

The construction of this speaker is significantly simpler than the Annular Teflon thermophone since it does not require that a flexible composite be made. It is prone to overheating problems, however, since the aluminum CNT panel is highly conductive to heat. 


\section{Annular Loudspeaker Array}

This piece of equipment was an attempt to replicate the annular design of the previous CNT iterations for use in the PVC pipe. Six 1-1/4" loudspeakers are positioned around the outside of a ring that fits onto the end of the PVC ducting (Figure 106).

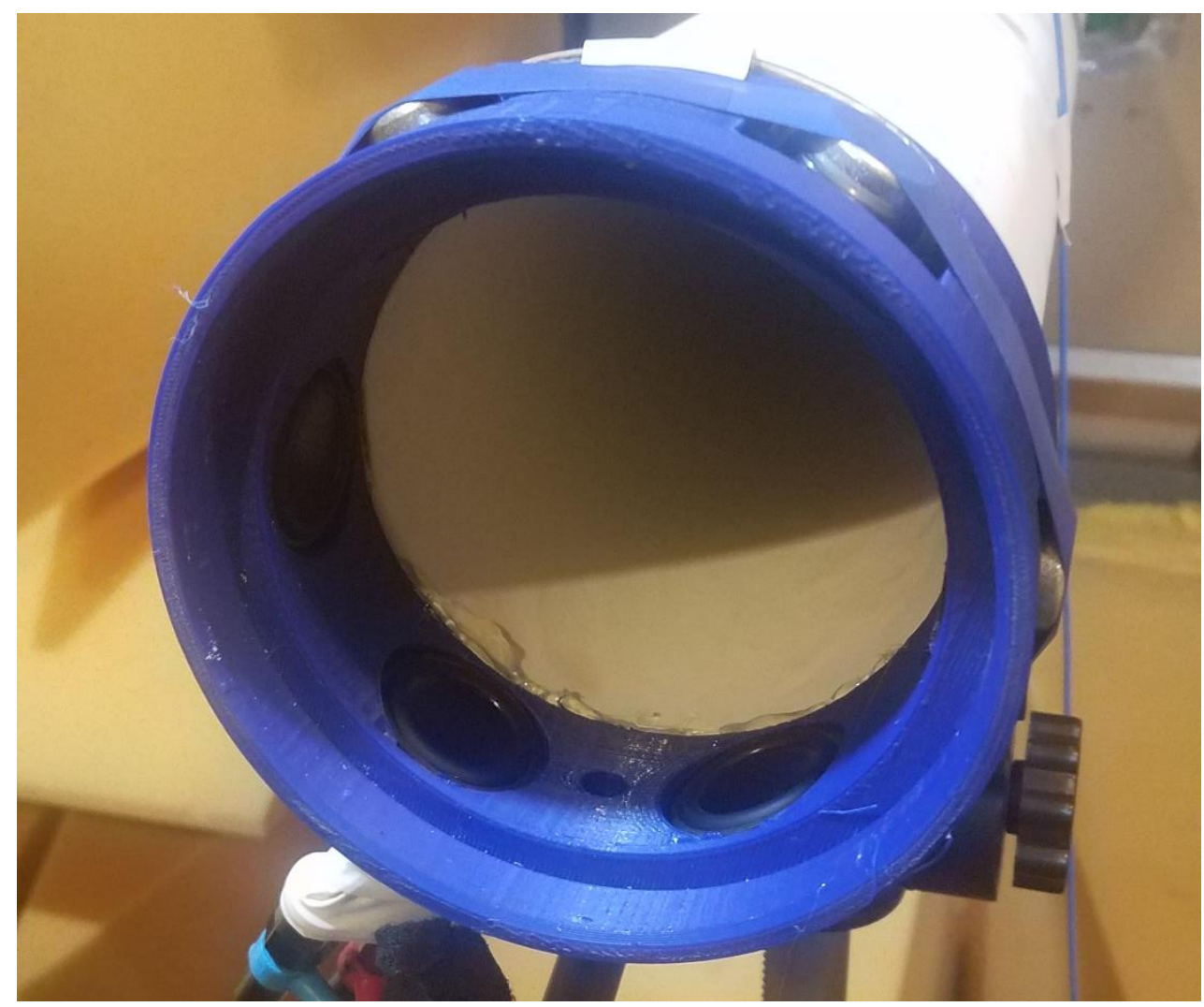

Figure 106: Design of Annular Loudspeaker Array

The loudspeaker set produces much higher output levels than the CNT thermophones and circumvents the usage of complicated signal processing in between the controller and actuator. Because of this this loudspeaker array was substituted for CNT during the custom controller testing so that as much complexity could be removed from the setup as possible. Performance of the loudspeaker array is anticipated to be slightly higher than CNT, although whether it produces a plane wave in the ducting as quickly as the CNT thermophones has not been answered. 


\section{Electronic Equipment}

\section{Digital Amplitude Modulator}

Traditional loudspeakers are considered linear devices in that the pressure produced by the speaker is linearly related to the voltage applied to it. For CNT, the pressure produced is linearly related to the power delivered to the speaker. Because of this, the frequency content of signals delivered to CNT thermophones using traditional audio equipment is doubled. The result of this is a sound that is very corrupted. In order to combat this problem, several signal processing techniques can be used to modify the signal, producing an intelligible sound from the CNT. Amplitude modulation is the technique used for this purpose in this case study.

The first piece of amplitude modulation equipment performed this task digitally. Centered around a raspberry $\mathrm{PI}$, this hardware is excellent for acoustic demos. After quite a bit of troubleshooting with ANC systems this controller was deemed unusable for running acoustic tests. The software-based nature of the controller caused the phase delay to be unpredictable. Since ANC is highly reliant on measurements and outputs having accurate phase, this modulator was retired.

\section{Analog Amplitude Modulator}

The second attempt to make an amplitude modulator used analog electronics. This version of the amplitude modulator did not suffer from inconsistent phase delay like its predecessor, making it suitable for acoustic testing. The circuit is housed on a breadboard and was constructed using electrical components common to radio equipment. Despite its small section here, this piece of equipment is critical to ANC testing with CNT and all cancellation results would not have been possible without it. 


\section{GLSV Order-Based Controller}

Early during the project, Great Lakes Sound and Vibration was contracted to build an ANC controller to be used on this, and other, ANC projects at MTU. The controller was delivered in several stages of completeness throughout the course of the project. When it was first used for testing, the controller was still in a prototype state, and required the support of one of the GLSV engineers for use (Figure 107). Later in the project's timeline a polished and complete version of the controller would be delivered.

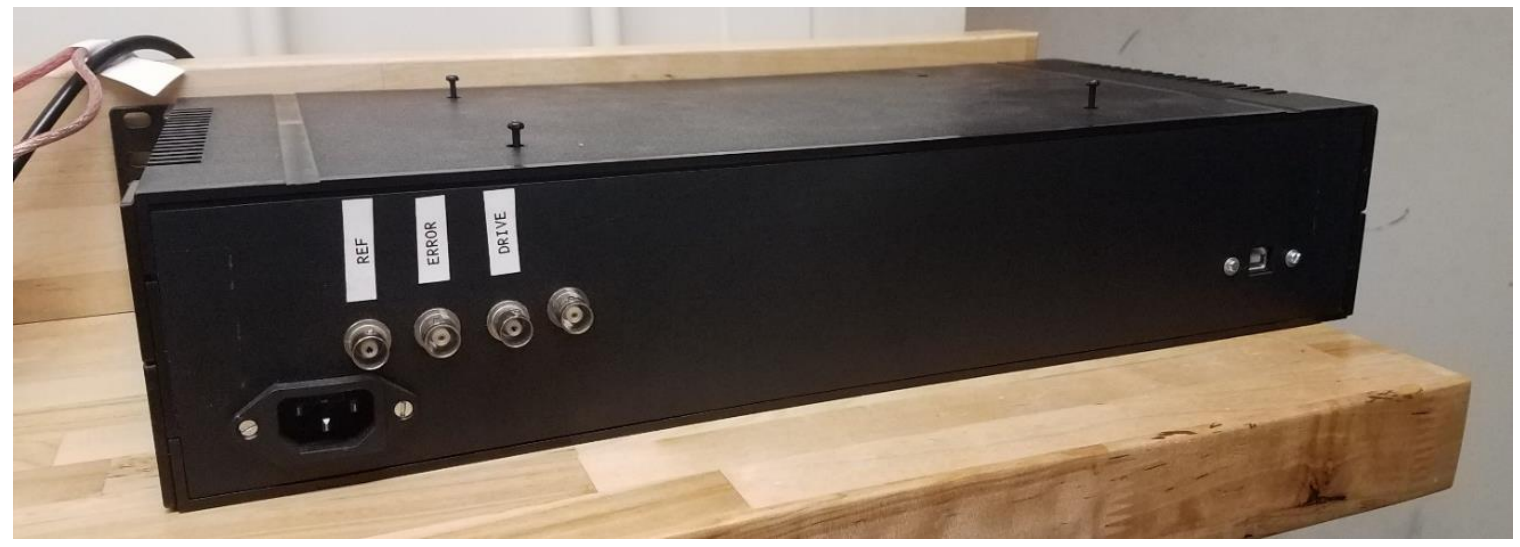

Figure 107: First Edition of the GLSV Order Based ANC Controller

Under the hood of this controller is a single frequency FXLMS algorithm. The controller acquires a square pulse from a tachymeter and produces a phase-locked sine wave at an arbitrary order. This sine wave is then used as the reference signal within the FXLMS algorithm. Multiple orders can be cancelled simultaneously in this way.

\section{Tiger WIFI-S Controller}

The Tiger WIFI-S is the second controller purchased for use in this case study. It was developed by Causal Systems and manufactured by Antysound. Selecting this controller over several competitors was mainly due to the company's affiliation with Dr. Colin Hansen, a professor at the University of Adelaide who is a prominent researcher in ANC. The controller is capable of single channel cancellation on either tonal or broadband noise using the FXLMS algorithm. Performance of the controller proved very successful at cancelling both tonal and broadband noise in the free field but showed only limited success in ducting tests. After identifying that acoustic feedback neutralization issues were limiting system performance in the latter case, testing with the Tiger WIFI-S concluded in lieu of pursuing a new controller. 


\section{cRIO FPGA Controller}

This controller is the last in the line of ANC controllers in this case study. It runs on a National Instruments cRIO 9031 platform and uses custom labView code for control. Two $\mathrm{NI} \mathrm{I/O} \mathrm{modules} \mathrm{are} \mathrm{used} \mathrm{in} \mathrm{the} \mathrm{cRIO} \mathrm{chassis,} \mathrm{the} \mathrm{NI9234} \mathrm{digital} \mathrm{input} \mathrm{card,} \mathrm{and} \mathrm{the} \mathrm{NI9260}$ digital output card.

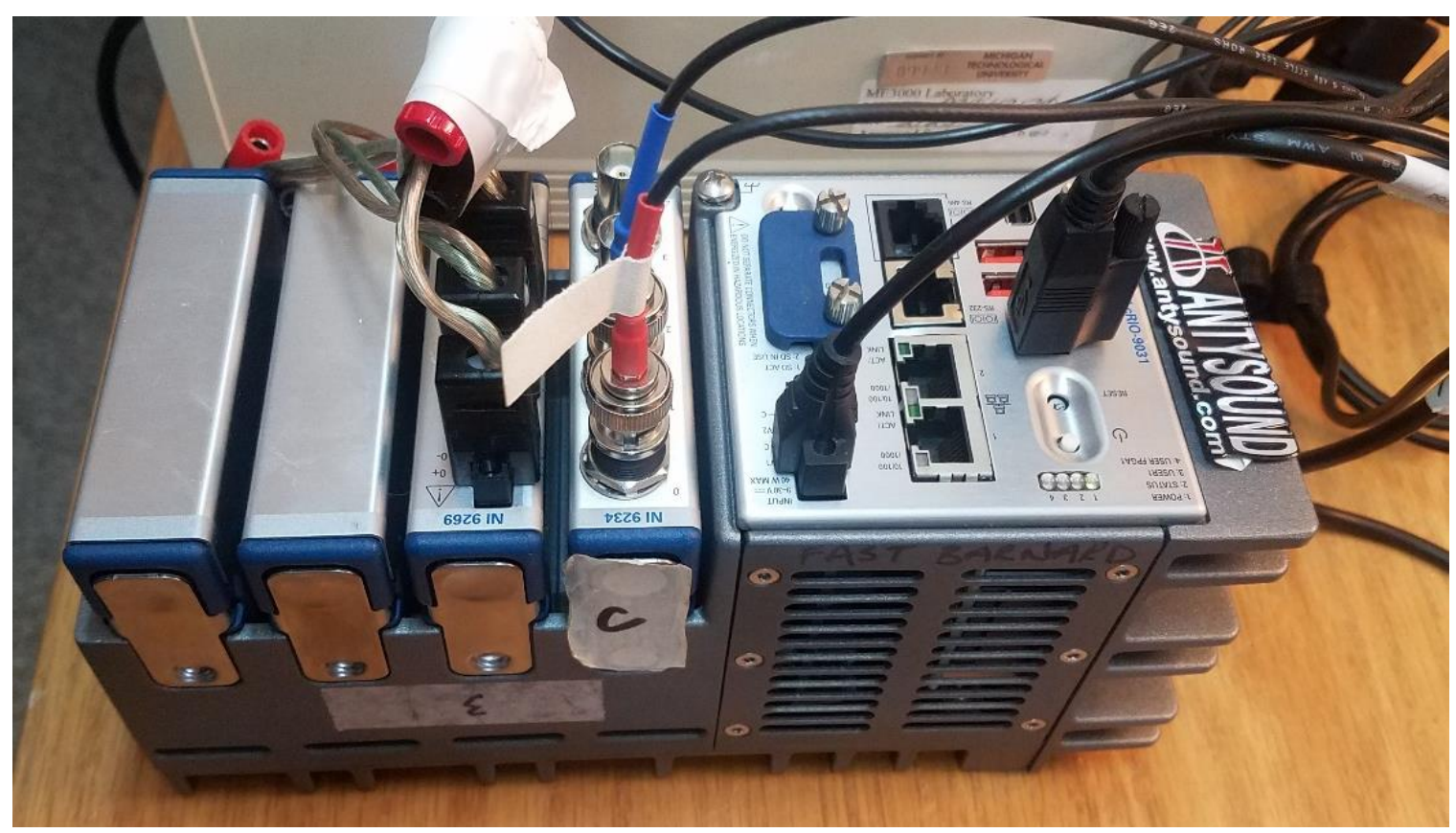

Figure 108: cRIO 9031 Configured to Serve as an ANC Controller

The algorithm implemented for ANC is the time-domain FXLMS algorithm with feedback neutralization. Extensive testing was done on this controller and it was found to be very effective at tonal attenuation as well as broadband attenuation in the free field. Effectiveness in ducted environments shows no improvement over the Tiger WIFI-S in terms of broadband cancellation levels achievable. 